\title{
Der Einfluss von Stickstoff und Trockenheit auf die Vitalität einer Hainbuchen- und Kiefernverjüngung
}

\author{
Dissertation \\ zur Erlangung des Doktorgrades \\ der Fakultät für Forstwissenschaften und Waldökologie \\ der Georg-August-Universität Göttingen \\ vorgelegt von
Martin Listing \\ geboren in Halle a. d. Saale
}

Göttingen, 2019 
1. Gutachter: Prof. Dr. Johannes Eichhorn

2. Gutachter: Prof. Dr. Christian Ammer

Tag der mündlichen Prüfung: 11. April 2019 


\section{Inhaltsverzeichnis}

1 Einleitung $\quad 11$

1.1 Resilienztheorie . . . . . . . . . . . . . . . . . . . 11

1.1.1 Störungen und Schwellenwerte . . . . . . . . . . . . . . . . 12

1.1.2 Vom Standortsfaktor zur Störung . . . . . . . . . . . . . . . . . 12

1.1.3 Trockenheit als Störung im Hessischen Ried . . . . . . . . . . . . . . . 13

1.1.4 Versuchsbedingte Störungen . . . . . . . . . . . . . . . . 13

1.1.5 Anpassung an Umweltfaktoren . . . . . . . . . . . . . . . . . 15

1.2 Versuchsmotivation . . . . . . . . . . . . . . . . . . . 16

1.3 Biokohle . . . . . . . . . . . . . . . . . . . 17

1.3.1 Bodenphysikalische Wirkungen von Biokohle . . . . . . . . . . . 17

1.3.2 Bodenchemische Wirkungen von Biokohle . . . . . . . . . . . . . . . 17

1.3.3 Forschung zur Anwendung im forstlichen Bereich . . . . . . . . . . . . . 18

1.4 Untersuchungsziel und Hypothesen . . . . . . . . . . . . . . . . . 18

2 Material und Methoden $\quad 19$

2.1 Standort . . . . . . . . . . . . . . . . . . . . . 19

2.1.1 Lage, Relief, Exposition . . . . . . . . . . . . . . . . . . 19

2.1 .2 Klima und Witterung . . . . . . . . . . . . . . . . . . . . . . . . . . . . . . .

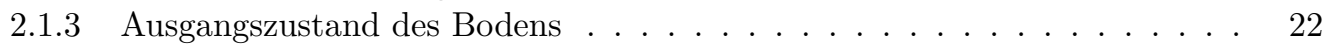

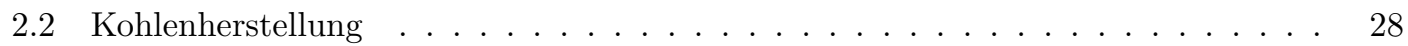

2.2.1 Ausgangssubstrate . . . . . . . . . . . . . . . . . . . . . . . . . . . . . . . . . . .

2.2.2 Nachbehandlung der Kohlen . . . . . . . . . . . . . . . 28

2.2.3 Physikalische und chemische Eigenschaften der Biokohlen im Versuch . . 29

2.3 Anlage des Feldversuches . . . . . . . . . . . . . . . . . . . 30

2.3.1 Versuchsdesign des Feldversuches . . . . . . . . . . . . . . . . . 30

2.3.2 Vorbereitung und Bepflanzung des Versuchsfeldes . . . . . . . . . . . . . 30

2.3.3 Zusammenlegung von Versuchsvarianten . . . . . . . . . . . . . . 32

2.4 Messmethoden und -verfahren . . . . . . . . . . . . . . . . . . . . . . . . . . . . . . . . . . .

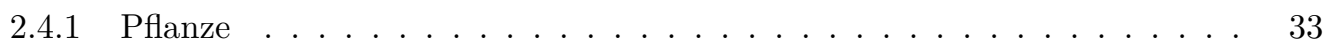

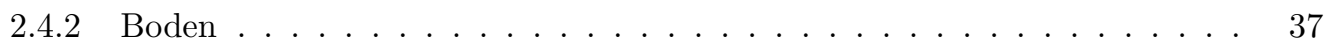

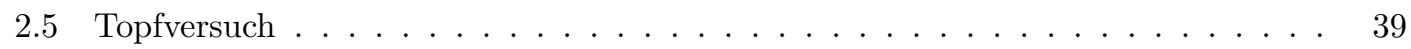

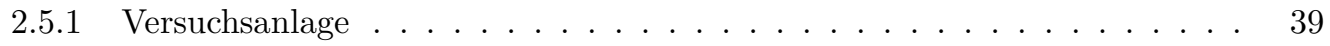

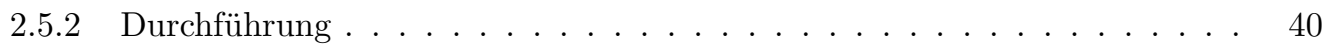

2.6 Extraktionsversuch . . . . . . . . . . . . . . . . . . . 40

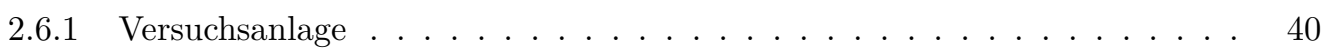

2.6 .2 Durchführung . . . . . . . . . . . . . . . . . 41

2.7 Sickerwassermodellierung . . . . . . . . . . . . . . . . . . . . . . . . . . . . . . . . .

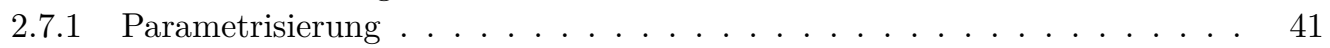

2.7 .2 Validierung . . . . . . . . . . . . . . . . . . . 42

2.8 Statistik . . . . . . . . . . . . . . . . . . . . . 44 
3 Ergebnisse

3.1 Umweltfaktor Trockenheit . . . . . . . . . . . . . . . . . . . . . . . . . 45

3.2 Änderung der Retentionseigenschaften des Bodens durch den Eintrag von Biokohle 46

3.2.1 Retentionskurven und daraus abgeleitete Parameter . . . . . . . . . . . . 46

3.2.2 Gravimetrischer Bodenwassergehalt in Ober- und Unterboden . . . . . . . 47

3.2 .3 Trockenrohdichte . . . . . . . . . . . . . . . . . . . . . 48

3.2.4 Hydrophobe Bodeneigenschaften . . . . . . . . . . . . . . . . . . 49

3.2 .5 Sickerwassermodellierung . . . . . . . . . . . . . . . . 50

3.3 Umweltfaktor Stickstoff . . . . . . . . . . . . . . . . . . . . . . . . . . 51

3.3 .1 Stickstoffeinträge . . . . . . . . . . . . . . . . . . 51

3.3.2 Stickstoff- und Kohlenstoffvorrat im Boden . . . . . . . . . . . . . . . . 51

3.3.3 Stickstoffaustrag . . . . . . . . . . . . . . . . . . . . . 52

3.3.4 Begleitvegetation auf den Versuchsparzellen . . . . . . . . . . . . . . . . . 59

3.3.5 Zusammenfassung Umweltfaktor Stickstoff . . . . . . . . . . . . . . . . . . 62

3.4 Anpassungsreaktionen der Bäume . . . . . . . . . . . . . . . . . . 63

3.4.1 Überlebenszahlen . . . . . . . . . . . . . . . . . . . . . . . . . . . 63

3.4.2 Blatt-/Nadelzustand und Blattmorphologie . . . . . . . . . . . . . 67

3.4.3 Spross- und Wurzelmorphologie . . . . . . . . . . . . . . . . . . . . 71

3.4.4 Elementgehalte der Kompartimente Blatt/Nadel und Feinwurzel . . . . . 78

3.4.5 Physiologische Indikatoren _. . . . . . . . . . . . . . . . . 83

3.5 Zusammenfassung der wichtigsten Ergebnisse . . . . . . . . . . . . . . . . . 84

3.5.1 Zeitliche Skalierung der Indikatoren und Effekte . . . . . . . . . . . . 84

3.5.2 Übersicht über die Hauptergebnisse . . . . . . . . . . . . . . . . . . 85

\section{Diskussion}

4.1 Einfluss von Biokohle auf Bodenphysik und Wasserhaushalt einschließlich Folgen der Bodenbearbeitung . . . . . . . . . . . . . . . . . . 87

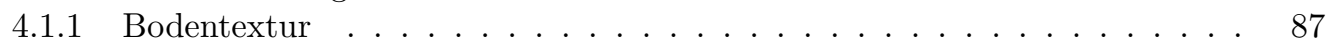

4.1 .2 Trockenrohdichte . . . . . . . . . . . . . . . . . . 87

4.1.3 Wasserspeicherfähigkeit . . . . . . . . . . . . . . . . . . . 88

4.1.4 Hydrophobie der Bodenoberfläche . . . . . . . . . . . . . . . . . . . 898

4.2 Einfluss von Biokohle auf den Stickstoffhaushalt . . . . . . . . . . . . . . . . . . 91

4.2.1 Einfluss der Bodenbearbeitung auf die Stickstoffmineralisierung . . . . . . 91

4.2.2 Stickstoffeintrag durch die Biokohlen . . . . . . . . . . . . . . . . . . 91

4.2.3 Stickstoffmineralisierung, -immobilisierung und Stickstoffaustrag . . . . . 92

4.2.4 Stickstoffflüsse und -vorräte . . . . . . . . . . . . . . . . . . . . . . 95

4.3 Wirkungen der Biokohle . . . . . . . . . . . . . . . . . . . . . . . . 98

4.3.1 Begleitvegetation . . . . . . . . . . . . . . . . . . . 98

4.3.2 Stickstoffaufnahme durch Hainbuche und Kiefer . . . . . . . . . . . . . . . 98

4.3.3 Andere Makronährstoffe . . . . . . . . . . . . . . . . . . . . . . . . . . . . 99

4.3.4 Schutz vor Trockenstress . . . . . . . . . . . . . . . . . . . . . . . . 99

4.3.5 Wirkung der Biokohle auf die Vitalität von Hainbuche und Kiefer . . . . 101

4.3.6 Wechselwirkungen von Stickstoffverfügbarkeit und Trockenheit . . . . . . 107

4.4 Zusammenfassende Betrachtungen zur Resilienz von Hainbuche und Kiefer . . . . 108

4.4 .1 Störungen . . . . . . . . . . . . . . . . . . . . . . . . . . . . . 108

4.4.2 Wirkungen der Störungen auf die Resilienz . . . . . . . . . . . . . . . . 110

4.4.3 Resilienzeigenschaften der Baumarten Hainbuche und Kiefer . . . . . . . 112

4.4.4 Die langfristige Entwicklung der Resilienz . . . . . . . . . . . . . . . . . . 113 
4.5 Methodendiskussion und Indikatorenauswahl . . . . . . . . . . . . . . . . 114

4.5.1 Allgemeine Methodendiskussion . . . . . . . . . . . . . . . . 114

4.5 .2 Indikatorenauswahl . . . . . . . . . . . . . . . . . . . . . . 115

5 Zusammenfassung $\quad 117$

5.1 Die wichtigsten Ergebnisse . . . . . . . . . . . . . . . . . . 117

5.2 Hypothesendiskussion . . . . . . . . . . . . . . . . . . . . 119

5.3 Unterschiede zwischen Holz- (HK) und Spelzenkohle (SK) . . . . . . . . . . . . . 120

5.4 Rückschlüsse für die forstliche Praxis und Ausblick . . . . . . . . . . . . . . . . 120

$\begin{array}{ll}\text { Literatur } & 123\end{array}$

$\begin{array}{lr}\text { A Anhang } & 139\end{array}$

$\begin{array}{lr}\text { B Abkürzungsverzeichnis } & 149\end{array}$ 


\section{Abbildungsverzeichnis}

1.1 Systemstabilität nach Walker und Salt (2012) . . . . . . . . . . . . . . . 11

1.2 Standorts- und Umweltfaktoren . . . . . . . . . . . . . . . . . . . . . 12

2.1 Lage der Versuchsfläche in Südhessen . . . . . . . . . . . . . . . . . . . . . . 19

2.2 Relief der Versuchsfläche . . . . . . . . . . . . . . . . . . . . . . 20

2.3 Retentionskurven des Ober- und Unterbodens der Nullvariante . . . . . . . . . . 23

2.4 pH-Wert, potenzielle Austauschkapazität, Calcium- und Magnesiumvorrat der Nullflächen vor Versuchsanlage . . . . . . . . . . . . . . . . . . . 25

2.5 Feinbodenvorrat, C/N-Verhältnis, Kohlenstoff- und Stickstoffvorrat der Nullflächen vor Versuchsanlage . . . . . . . . . . . . . . . . . . . 25

2.6 Das Bodenprofil auf der Versuchsfläche . . . . . . . . . . . . . . . . . . . 27

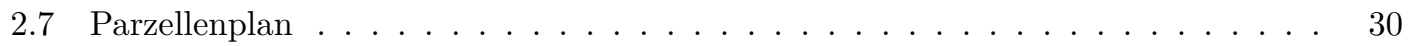

2.8 Flächenzustand vor Kohleneinarbeitung . . . . . . . . . . . . . . . . . . . . . . . . . . . . . . .

2.9 Pflanzplan . . . . . . . . . . . . . . . . . . . . . . . . . 32

2.10 Beispiel der Vitalitätskategorie „starker Blattverlust“ bzw. „Nadelverbraunung“ . 33

2.11 Topfversuch im Gewächshaus . . . . . . . . . . . . . . . . . . . . 39

2.12 Vergleich der gemessenen mit den modellierten Bodenwassergehalten in $100 \mathrm{~cm}$ Bodentiefe der Jahre 2013 - 2016 auf der Level II-Fläche Hessisches Ried . . . . . 42

2.13 Vergleich der gemessenen mit den modellierten Bodenwassergehalten in $20 \mathrm{~cm}$ Bodentiefe der Jahre 2013 - 2016 auf der Level II-Fläche Hessisches Ried . . . . . 43

2.14 Vergleich der gemessenen mit den modellierten Bodenwassergehalten in $20 \mathrm{~cm}$ und $100 \mathrm{~cm}$ Bodentiefe der Jahre 2015 und 2016 der Nullvariante . . . . . . . . . . . 43

3.1 Bodenwassergehalt und Bodentemperatur im Oberboden im Zeitraum 23.06.2015 bis 13.06.2017 auf einer Parzelle der Nullvariante. . . . . . . . . . . . . . . 45

3.2 Retentionskurven des Ober- und Unterbodens der Versuchsvarianten. . . . . . . . 46

3.3 Änderung der Trockenrohdichte mit der Bodenbearbeitung . . . . . . . . . . . . 48

3.4 Kumulierte Sickerwassermengen in mm der Jahre 2014 - 2018 . . . . . . . . . . . 50

3.5 Kohlenstoff- und Stickstoffvorräte im Jahr 2015 im Oberboden . . . . . . . . . . 52

3.6 Nitrat- und Ammoniumkonzentration des Sickerwassers in den Jahren 2015 - 201753

3.7 Nitrat- und Ammoniumausträge während der Vegetationszeiten und Nichtvegetationszeiten $2015-2017$. . . . . . . . . . . . . . . . . 54

3.8 Nitratkonzentration des Sickerwassers im Topfversuch . . . . . . . . . . . . . 56

3.9 Ammoniumkonzentration des Sickerwassers im Topfversuch . . . . . . . . . . 56

3.10 Mittlere Nitrat-N- und Ammonium-N-Konzentration im Extraktionsversuch . . . 58

3.11 Deckungsgrad der Begleitvegetation auf den Versuchsparzellen, 2014 - 2017 . . . 59

3.12 Parzellenansicht der Nullvariante und der Variante HK+N+Ko im Jahr 2014 . . 59

3.13 Mittlere Überlebensraten von Hainbuche und Kiefer im Feldversuch . . . . . . . 63

3.14 Mittlere Überlebensrate der 2016 gepflanzten Kiefer . . . . . . . . . . . . . . . . 64

3.15 Mittlere Lebensdauer im Topfversuch im Jahr 2017. . . . . . . . . . . . . . . . 66

3.16 Blattverlust der Hainbuche in den Jahren 2015 - 2017 . . . . . . . . . . . . . . . 67

3.17 Neuer Blattaustrieb im August $2016 \ldots$. . . . . . . . . . . . . . . . 68 
3.18 Nadelverfärbung der 2015 gepflanzten Kiefer im Jahr 2015 und der 2016 gepflanzten Kiefer im Jahr 2016. . . . . . . . . . . . . . . . . . . .

3.19 Einzelblattfläche und -trockenmasse der Hainbuche im Feldversuch . . . . . . . .

3.20 Zuwachs des Wurzelhalsdurchmessers von Hainbuche (Feldversuch) und Jahrestrieblänge Kiefer im Topfversuch . . . . . . . . . . . . . . . . . . . . .

3.21 Spross-Wurzel-Verhältnis von Hainbuche im Topf- und Feldversuch . . . . . . . .

3.22 Spross-Wurzel-Verhältnis der Trockenmassen der Kiefer im Topf- und Feldversuch

3.23 Anteil der Feinwurzeltrockenmasse an der Gesamttrockenmasse der Hainbuchen im Topf- und Feldversuch . . . . . . . . . . . . . . . . . . . .

3.24 Anteil der Feinwurzeltrockenmasse an der Gesamttrockenmasse der Kiefer im Topf- und Feldversuch . . . . . . . . . . . . . . . . . . . . .

3.25 Mykorrhizierungsgrad und Anteil trockener Feinwurzelspitzen von Hainbuche und Kiefer im Oktober 2016. . . . . . . . . . . . . . . . . . . . . 75

3.26 Stickstoffgehalte und SPAD-Werte der Hainbuchenblätter 2014 - 2016. . . . . . . 78

3.27 Stickstoffgehalte und SPAD-Werte der Hainbuchenblätter im Topfversuch. . . . . 79

3.28 Stickstoffgehalte der Hainbuchen-Feinwurzeln und der Kiefernkompartimente in den Jahren 2015 und 2016. . . . . . . . . . . . . . . . . . . . . . . . .

3.29 Blattwassergehalte und stomatäre Leitfähigkeit der Hainbuchenblätter während der Vegetationszeiten 2015 und 2016. . . . . . . . . . . . . . . . . .

4.1 Entwicklung der Trockenrohdichte im Oberboden der Nullvariante nach Bodenbearbeitung ..........................

4.2 Stickstoffflüsse und -vorräte der Nullvariante . . . . . . . . . . . . . . . . . .

4.3 Stickstoffflüsse und -vorräte der Variante HKpur . . . . . . . . . . . . . . . . .

4.4 Stickstoffflüsse und -vorräte der Variante $\mathrm{HK}+\mathrm{N}+\mathrm{Ko}$. . . . . . . . . . . . . . . .

4.5 Dynamik von Störungen und Wirkungen . . . . . . . . . . . . . . . . .

4.6 Systemzustand und Resilienzveränderungen am Beispiel des Feldversuches . . . .

4.7 Zeitliche Darstellung der Störungen und die Messzeitpunkte der Indikatoren. . .

A.1 Thermopluviogramme der Monate und Vegetations- und Nichtvegetationszeiten (VZ) der Jahre 2014 bis 2017. Relative Temperatur- und Niederschlagsabweichungen im Vergleich zur Referenzperiode 1961 - 1990 . . . . . . . . . . . . . . .

A.2 Gravimetrischer Wassergehalt in Ober- und Unterboden in den Monaten März 2016 bis März 2017 . . . . . . . . . . . . . . . . . . . . . . . 140

A.3 SPAD-Wert der Hainbuchenblätter im Topfversuch. . . . . . . . . . . . . . . . . . 143

A.4 SPAD-Wert der Hainbuchenblätter während der Vegetationszeit 2015. . . . . . . 143

A.5 Calciumgehalte der Hainbuchenblätter im Topf- und Feldversuch . . . . . . . . . 144

A.6 Magnesiumgehalte der Hainbuchenblätter im Topf- und Feldversuch . . . . . . . 144

A.7 Kaliumgehalte der Hainbuchenblätter im Topfversuch und Feldversuch . . . . . . 145

A.8 Phosphorgehalte der Hainbuchenblätter im Topfversuch und Feldversuch . . . . . 145

A.9 Calcium- und Magnesiumgehalte der Kiefernadeln im Feldversuch, 2015 und 2016146

A.10 Kalium- und Phosphorgehalte der Kiefernadeln im Feldversuch, 2015 und 2016 . 146 


\section{Tabellenverzeichnis}

2.1 Mittlere Monatstemperaturen der Jahre 2013 - 2017 und 1961 - 1990 . . . . . . 21

2.2 Monatliche Niederschlagssummen der Jahre 2013 - 2017 und 1961 - 1990 . . . . . 21

2.3 Korngrößenverteilung in Ober- und Unterboden auf einer Parzelle der Nullvariante 22

2.4 Trockenrohdichte der Nullvariante im Jahr 2014 (nach Bodenbearbeitung) . . . . 24

2.5 Bezeichnung der Versuchsvarianten . . . . . . . . . . . . . . . . . . 28

2.6 Physikalische Eigenschaften der puren Kohlesubstrate. . . . . . . . . . . . . . . . 29

2.7 Chemische Eigenschaften der Kohlesubstrate . . . . . . . . . . . . . . . . . . . . 29

2.8 Zusammmengelegte Versuchsvarianten . . . . . . . . . . . . . . . . . . 32

2.9 Boniturstufen und Beschreibung von Schadsymptomen an Blättern und Nadeln. . 40

3.1 Wassergehalt bei Feldkapazität und permanentem Welkepunkt und nutzbare Feldkapazität der Substrate in Ober- und Unterboden . . . . . . . . . . . . . . . 47

3.2 Mittlere volumetrische Bodenwassergehalte der Kohlevarianten normiert auf den Bodenwassergehalt der Nullvariante . . . . . . . . . . . . . . . . 47

3.3 Mittlere Trockenrohdichten in den Jahren 2014, 2015 und 2016 . . . . . . . . . . 48

3.4 Mittlere Versickerungszeit auf den Parzellen der Versuchsvarianten im Sommer 201549

3.5 Anzahl der wassergesättigten Töpfe in der Vorbereitungsphase des Topfversuches 49

3.6 Gesamtstickstoffgehalte der Kohlen, Eintragsmengen und Anteil des Eintrages am

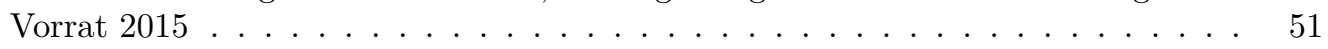

3.7 Jahressummen der Nitrat-N und Ammonium-N-Austräge 2015 - 2017 . . . . . . 55

3.8 Anteil der mineralischen Stickstoffausträge am Stickstoffeintrag über das Sickerwasser in Feld- und Topfversuch . . . . . . . . . . . . . . . . . . . 57

3.10 Mittlere Stickstofffestlegung in der Vegetation . . . . . . . . . . . . . . . . . . 60

3.9 Die häufigsten Arten der Begleitvegetation mit ihren mittleren Deckungsgraden auf den Versuchsparzellen in den Jahren 2014 - 2017. . . . . . . . . . . . . . 61

3.11 Überlebenszahlen im Feldversuch . . . . . . . . . . . . . . . . . . . . . 65

3.13 Zusammenfassung Stickstoffernährung für Hainbuche . . . . . . . . . . . . . . . . 82

3.14 Zeitliche Skalierung der Auswirkungen auf Boden und Pflanze . . . . . . . . . . . 84

3.15 Übersichtsliste über Effekte im Vergleich zur Nullvariante . . . . . . . . . . . . . 85

4.1 Übersicht über den Stickstoffeintrag mit der Kohle, das Mineralisierungspotenzial, das Bindungspotenzial für Stickstoff und die Stickstoffverfügbarkeit in den Jahren nach Versuchsbeginn . . . . . . . . . . . . . . . . . .

4.2 In dieser Arbeit beobachtete kurz-, mittel- und langfristige Pflanzenreaktionen auf Trockenstress ............................ 100

A.1 Korngrößenverteilung im Ober- und Unterboden . . . . . . . . . . . . . . . . . 140

A.2 Bodenchemie vor Kohleeinarbeitung. Elementgehalte . . . . . . . . . . . . . . . . 140

A.3 Gesamtstickstoffgehalte der Versuchsvarianten in den Jahren 2014 und 2015 . . . 141

A.4 Parameterwerte in Brook90 . . . . . . . . . . . . . . . . . . . . . . 142

A.5 Nährstoffverhältnisse in Hainbuchenblättern und Kiefernnadeln im Topf- und Feld-

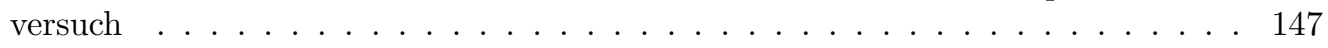




\section{Vorwort}

Diese Arbeit wurde im Rahmen eines durch das Hessischen Landesamtes für Naturschutz, Umwelt und Geologie über das Fachzentrum Klimawandel geförderten Projektes angefertigt. Die geförderten Forschungsprojekte sollen direkte und indirekte Auswirkungen des Klimawandels auf die Forstwirtschaft abschätzen und Maßnahmen für die Anpassung an die Folgen des Klimawandels entwickeln.

Ohne die Unterstützung zahlreicher Personen hätte diese Arbeit nicht realisiert werden können. Für die vielfältig erfahrene Hilfe möchte ich mich an dieser Stelle sehr herzlich bedanken.

Mein besonderer Dank gilt Prof. Dr. Johannes Eichhorn für die wissenschaftliche und methodische Unterstützung während der gesamten Dissertation. Für seine analytischen Anmerkungen und die Fähigkeit mich intrinsisch zu motivieren. Ich danke ebenso Prof. Dr. Ammer, der meine Doktorarbeit als Zweitgutachter betreut, für die konstruktiven Anregungen.

Für die vielfältige Unterstützung bin ich der Nordwestdeutschen Forstlichen Versuchsanstalt besonders verbunden. Allen beteiligten Mitarbeitern möchte ich für die zahlreichen fachlichen Hinweise und die organisatorische Unterstützung danken. Besonders erwähnen möchte ich Uwe Paar, Jan Evers, Nils König, Paul Schmidt-Walter, Jörg Weymar und Michael Spielmann.

Ohne die Hilfe einiger Studenten wären die Inventuraufnahmen und Probenahmen nicht möglich gewesen. Besonderen Anteil hat Alexander Busch, dessen Untersuchungsergebnisse zur Mykorrhizierung, im Rahmen seiner Masterarbeit, Eingang in diese Arbeit gefunden haben.

Den Mitarbeitern der Abteilung Forstbotanik und Baumphysiologie gebührt mein Dank für die Unterstützung mit Messgeräten, in der Probenvorbereitung und der fachlichen Hilfe bei den Untersuchungen zur Mykorrhizierung. Sonja Löffler vom Landeskompetenzzentrum Forst in Eberswalde für die intensive Hilfe bei den Biomarkeranalysen, deren Ergebnisse leider keinen Eingang in diese Arbeit gefunden haben. Claudia Kammann, Universität Geisenheim für die fachliche Beratung vor allem in der Anfangsphase des Versuches.

Meinen Eltern und Schwiegereltern danke ich für die moralische Unterstützung. Der größte Dank gilt Frauke für die unermüdliche Stärkung und Motivation. 


\section{Einleitung}

In dieser Arbeit wird die Reaktion einer forstlichen Verjüngungen auf die Umweltfaktoren Trockenheit und Stickstoffverfügbarkeit näher beschrieben. Pflanzen sind wie alle Organismen vielfältigen Umweltbedingungen ausgesetzt. Die Wirkung dieser Umweltbedingungen kann ebenso vielfältig sein und bis zum Absterben des Organismus reichen.

Einführend werden notwendige Begrifflichkeiten definiert und erläutert. Ein kurzer Überblick über den bisherigen Forschungstand zum Thema Biokohle soll anschließend zu den Arbeitshypothesen dieser Untersuchung überleiten.

\subsection{Resilienztheorie}

Um die komplexen Beziehungen zwischen Umwelt und Organismus zu beschreiben und zu bewerten, kann die Resilienztheorie ein Rahmenkonzept geben. Als Resilienz wird die Fähigkeit eines Ökosystems bezeichnet, Störungen zu kompensieren und seine Basisfunktionen weiterhin zu erfüllen, ohne seine grundlegende Organisationsstruktur zu ändern (Holling 1973; Walker und Salt 2012). Von dem lateinischen Begriff resilire (deutsch: zurückspringen, abprallen) abgeleitet, stellt es einen Organismus oder ein Ökosystem in das Zentrum der Betrachtung und beschreibt das Verhalten dieses Systems unter der Einwirkung von Umwelteinflüssen. Im Unterschied zur Stresstheorie wird den einwirkenden Umweltfaktoren keine positive (Eustress) oder negative (Distress) Wirkung zugeschrieben.

Wälder können im Sinne der Resilienztheorie nach Walker und Salt (2012) als komplexe adaptive Systeme beschrieben werden. Eigenschaften solcher komplexen adaptiven Systeme sind die unabhängige Interaktion von Teilkomponenten, ein stattfindender Selektionsprozess und die Zunahme der Variation mit der Zeit. Durch die Ortsgebundenheit sind Vorgänge der Optimierung und der Anpassung für Bäume überlebenswichtig (Holland 1992; Roloff 2005). Die Umwelteinflüsse aus der Vergangenheit sind teilweise bis heute wirksam und können das Anpassungsvermögen beeinflussen (Eichhorn et al. 2016).

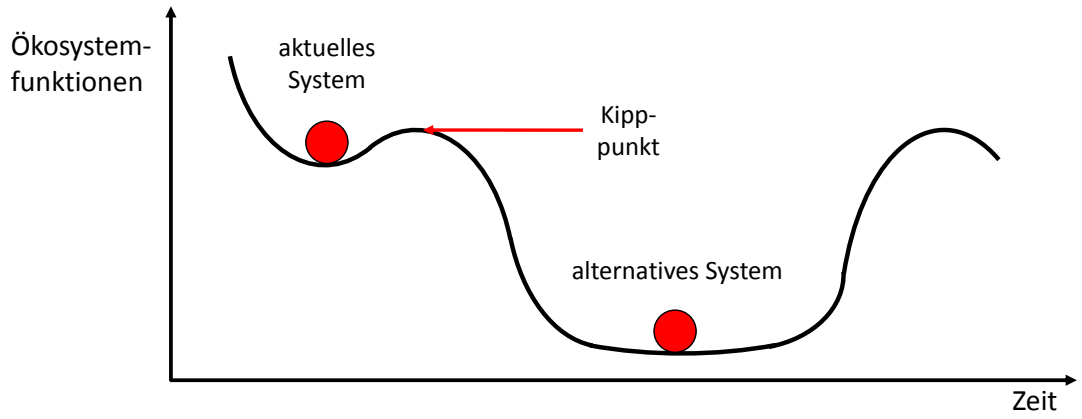

Abbildung 1.1: Systemstabilität nach Walker und Salt (2012)

Die Resilienztheorie soll hier einen Rahmen für die Bewertung der Systemstabilität bieten. Dazu bietet sich die Ball-in-der-Tasse Darstellung nach Walker und Salt (2012) an (siehe Abb. 1.1). 
Der aktuelle Zustand eines Ökosystems wird durch die Kugel dargestellt. Deren Lage wird durch Umwelteinflüsse (Störungen) bestimmt und kann durch spezifische Schlüsselindikatoren beschrieben werden.

Bei einer Grenzwertüberschreitung befindet sich die Kugel nicht mehr innerhalb der ursprünglichen Senke, das System geht in ein alternatives System über. Die Wandhöhe bzw. die Schwelle, die die Kugel überwinden muss, stellt die Stabilität des Ökosystems dar. Resilienzerhöhende bzw. -senkende Maßnahmen erhöhen bzw. senken den Tassenrand ab. Nach einer Resilienzerhöhung kann das Ökosystem stärkere Störungen kompensieren, ohne wesentliche Funktionen zu verlieren.

\subsubsection{Störungen und Schwellenwerte}

Schwellen- oder Grenzwerte beschreiben Grenzen zwischen unterschiedlichen Ökosystemzuständen. Unterhalb eines kritischen Wertes bleibt der aktuelle Systemzustand erhalten und Störungen werden kompensiert. Übersteigt der Schwellenwert diesen kritischen Wert (Kipppunkt), geht das System in einen alternativen Systemzustand über. Die Reaktion (die Systemantwort) eines Ökosystems auf Stressoren bzw. Störungen hängt sehr stark vom jeweiligen Kontext ab. Meist sind nicht alle Störungen bekannt und die Störungen treten häufig in Kombination und Wechselwirkung auf. So beeinflusst ein Nährstoffmangel bzw. Ungleichgewichte in der Nährstoffversorgung die Fähigkeit klimatische Störungen wie Trockenperioden oder Spätfrostereignisse zu ertragen (Kätzel et al. 2008).

Die Resilienz von Ökosystemen ist keine konstante Größe. Sie hängt von den beteiligten Arten und den Störungen ab. Zusätzlich ändert sie sich im Verlauf der Ontogenese (Individualentwicklung). So weisen junge Bäume aufgrund der kleinen Wurzelsysteme eine höhere Trockenstressgefährdung auf, als ältere Bäume. Eine Prämisse für diese Arbeit lautet daher, dass Waldökosysteme in der Kulturphase deutlich weniger resilient sind, als Wälder in der Optimalphase.

\subsubsection{Vom Standortsfaktor zur Störung}

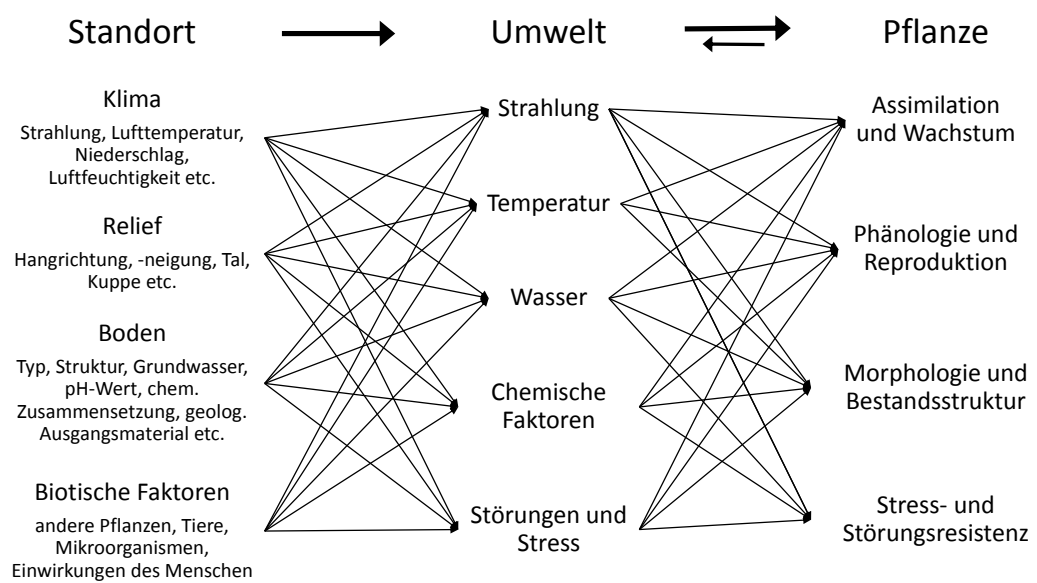

Abbildung 1.2: Standorts- und Umweltfaktoren. Verändert nach Sitte et al. (2002) 
Der Standort fasst in der Ökologie die herrschenden Umweltbedingungen an einem definierten Ort zusammen. Die abiotischen Standortsfaktoren Klima, Relief und Boden werden im Rahmen der Standortskartierung aufgenommen (Sitte et al. 2002; Standortskartierung 2003). Sie wirken zusammen mit den biotischen Standortsfaktoren über die Umweltfaktoren auf Pflanzen und andere Organismen (siehe Abb. 1.2).

Pflanzen reagieren auf diese Umweltfaktoren mit Wachstum, Entwicklung, struktureller Ausprägung und Resistenz. Wenn Umweltfaktoren Stresszustände auslösen, wirken sie als Stressfaktoren oder Störungen (Sitte et al. 2002), die selten isoliert auftreten. Das Wechselspiel zwischen Störungen führt zu einer Einengung des Lebensraumes von Pflanzenarten (Larcher 1994).

In dieser Arbeit werden die Wirkungen der Störungen Trockenheit bzw. Dürre, die Bodenbearbeitung, eine sehr hohe Stickstoffverfügbarkeit sowie mögliche Wechselwirkungen zwischen den Stressoren auf die Pflanzen näher untersucht. Weitere abiotische Stressoren wie Hitze, Strahlung oder Frost und biotische Stressoren wie Maikäfer, Mäuse oder Pilze sind nicht Gegenstand dieser Untersuchung.

\subsubsection{Trockenheit als Störung im Hessischen Ried}

Eine unzureichende Wasserversorgung durch Bodentrockenheit bei gleichzeitig hoher Verdunstung führt zu einer angespannten Wasserbilanz eines Pflanzenorganismus. Aufgrund der sich, im Gegensatz zu anderen Stressoren, langsam anbahnenden Belastung ist die zeitliche Dimension der darauf folgenden Pflanzenreaktionen bis hin zum Pflanzentod ein wichtiger Faktor (Larcher 1994).

Im Hinblick auf sich ändernde Klimabedingungen ist die nähere Untersuchung der Trockenstresstoleranz von Baumarten wie Kiefern und Hainbuchen von besonderem Interesse. Diese Baumarten gelten als verhältnismäßig gut angepasst gegenüber länger anhaltenden Trockenperioden (Kätzel et al. 2008; Ellenberg und Leuschner 2010).

Die Wälder in der Rhein-Main-Ebene sind seit Jahrzehnten einer Destabilisierung durch vielfältige Umweltfaktoren ausgesetzt. Neben natürlichen Einflüssen wie hohen Temperaturen und unregelmäßigen Niederschlägen in der Vegetationsperiode wirkt durch die Lage in der Metropolregion Main-Neckar eine Vielzahl von anthropogenen Umweltfaktoren auf die Wälder ein und führt zu Waldauflösungserscheinungen. Durch hohe Trinkwasserfördermengen wurde seit den 70er Jahren des 20. Jahrhunderts der Grundwasserstand deutlich abgesenkt und dadurch eine tiefgreifende Standortsveränderung herbeigeführt. Im Zuge des globalen Klimawandels werden sich die räumlichen und zeitlichen Muster der Niederschläge signifikant verändern (Karl et al. 1995; Dore 2005; Solomon et al. 2007). Die Häufigkeit und Intensität von Trockenperioden wird möglicherweise ansteigen (Ciais et al. 2014). Für die Region Südhessen wird eine Zunahme von Extremereignissen wie Starkregen, Hitzewellen und langanhaltende Trockenperioden prognostiziert (Kemper et al. 2011; NW-FVA 2013).

\subsubsection{Versuchsbedingte Störungen}

\subsubsection{Bodenbearbeitung}

Im Zuge der Vorbereitung des Versuchs wurde die Versuchsfläche vollflächig gemulcht. Dabei wurde der Humus und ein Teil der Strauchvegetation zerkleinert und in den Oberboden eingearbeitets. Kurz vor der Pflanzung sind alle Parzellen gefräst und gewalzt worden. Diese intensive Bodenbearbeitung hat enorme Auswirkungen auf Parameter der Bodenphysik (Trockenrohdichte, Porenvolumen) und wird in der Versuchsauswertung daher als versuchsbedingte Störung genauer untersucht und diskutiert. 


\subsubsection{Stickstoffeintrag mit Auswirkungen auf die Stickstoffverfügbarkeit}

Durch die Einarbeitung von stickstoffbehandelten Kohlesubstraten in den Oberboden wurde der Stickstoffhaushalt des ursprünglich stickstoffarmen Standortes durch einen Stickstoffeintrag stark verändert. In der Folge ergeben sich Hinweise auf eine deutlich erhöhte Stickstoffverfügbarkeit.

\begin{tabular}{|c|}
\hline 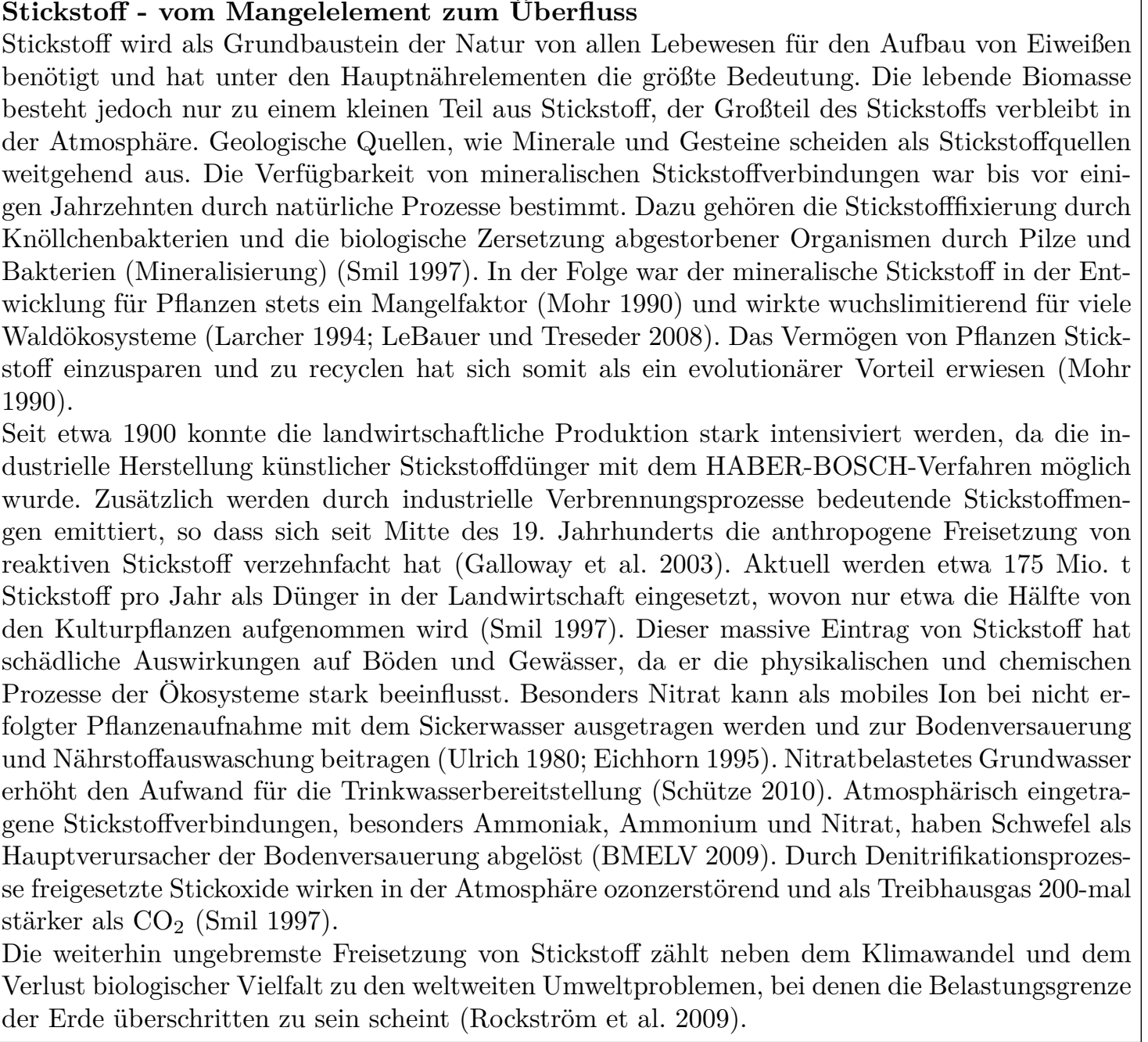 \\
\hline
\end{tabular}

Auswirkungen einer erhöhten Stickstoffverfügbarkeit auf Pflanzen Die Auswirkungen einer erhöhten Stickstoffverfügbarkeit auf die Pflanzen sind vielfältiger Natur. Der Massenzuwachs von Pflanzen wird wie bereits erwähnt vor allem durch das Stickstoffangebot limitiert. Gravierender Stickstoffmangel führt zu kümmerlichem Pflanzenwuchs, kleinzelligem Gewebe und derben Zellwänden (Stickstoffmangelsklerose), auch Prozesse der Reproduktion und Seneszenz setzen früher ein (Larcher 1994). Eine erhöhte Stickstoffverfügbarkeit kann in stickstofflimitierten Systemen daher zunächst zu einer Steigerung der pflanzlichen Primärproduktion führen. Eine Überversorgung mit Stickstoff kann jedoch ebenso starke Einbußen an Ertrag und Pflanzenqualität nach sich ziehen (Hock 1988). Die Pflanzenreaktionen folgen hinsichtlich der Stickstoffverfügbarkeit einer Optimumkurve. 
Für die Waldbodenvegetation sind durch Stickstoffeinträge veränderte Konkurrenzbeziehungen zwischen stickstoffangepassten (nitrophilen) Arten und Arten stickstoffärmerer Standorte nachgewiesen (Roloff 1989). Damit einhergehend sinkt die Vielfalt der Bodenvegetation (Eichhorn 1995). Eine erhöhte Stickstoffverfügbarkeit geht mit veränderten Spross-Wurzel-Verhältnissen der Pflanzenbiomasse und geringeren Feinwurzelbiomassen (Rapp 1991) einher. Zusammen mit Nährstoffungleichgewichten können Zuwachslimitierungen und Vitalitätseinbußen entstehen (Prietzel et al. 2008; Braun et al. 2010). Auch die Abundanz und die Vielfalt von Mykorrhizapilzen, die eine große Bedeutung für die Nährstoff- und Wasseraufnahme von Waldbäumen haben, ist durch steigende Stickstoffeinträge gefährdet (Göbl und Platzer 1967; Rapp 1991; De Witte et al. 2017).

Stickstoffaufnahme Nitrat- und Ammoniumionen werden wie andere mineralische Nährstoffe aus dem Boden aufgenommen, nehmen jedoch unter den Pflanzennährstoffen eine besondere Rolle ein. Der Stickstoffbedarf der Pflanzen ist im Verhältnis zu anderen Nährstoffen am höchsten (Scheffer 2002). Etwa 50 - $60 \%$ der gesamten Ionenaufnahme einer Pflanze geht auf die Aufnahme von Stickstoff in unterschiedlichen Verbindungen zurück (Evers 1964). Bei günstigem Boden-pH im Wurzelraum können die meisten Pflanzen grundsätzlich sowohl Nitrat als auch Ammonium aufnehmen und so ihren Stickstoffbedarf decken (Salsac 1987). Die Buche besitzt zwar eine Präferenz für die Aufnahme von Ammonium, kann die Stickstoffaufnahme aber bei überwiegendem Nitratangebot auch auf Nitrat umstellen (Paar 1994). Die aufgenommene Stickstoffform beeinflusst die Ionenbilanz und den Energie- und Phytohormonhaushalt, da Kationen (Ammonium) und Anionen (Nitrat) aufgenommen werden (Salsac 1987). Die Aufnahme von Nitrat und anschließender Reduktion bedeutet einen etwa dreifach höheren Energieaufwand als die direkte Aufnahme von Ammonium.

\subsubsection{Anpassung an Umweltfaktoren}

Das Anpassungspotenzial von Pflanzen beschreibt die Fähigkeit auf Umweltbedingungen physiologisch reagieren zu können. Sie ist im Wesentlichen genetisch festgelegt und ermöglicht u.a. die Elastizität bzw. ökologische Stabilität von Wäldern. Innerhalb des physiologischen Toleranzbereiches, in dem das Überleben unter den gegebenen Umweltfaktoren gerade noch möglich ist, kann eine Konditionierung die Widerstandsfähigkeit von Organismen sowohl erhöhen als auch senken. Die Art dieser Anpassung ist von Stärke und Dauer der Störung abhängig (Larcher 1994) und kann sowohl reversibel als auch irreversibel sein (Sitte et al. 2002).

Die permanent ablaufenden Anpassungsprozesse finden auf unterschiedlichen zeitlichen Skalen statt und können entsprechend differenziert werden. Die evolutive Anpassung ist vererbbar, demzufolge langfristig und für den Besiedelungserfolg einer Art ausschlaggebend. Prozesse der modifikativen Anpassung sind für das Überleben eines Einzelbaumes unter spezifischen Standortsbedingungen verantwortlich, langfristig und nicht vererbbar. Dazu können Anpassungsreaktionen auf morphologischer Ebene wie etwa Zellwandverstärkungen oder eine veränderte Biomasseallokation gezählt werden. sDie modulative Anpassung an Umweltfaktoren ist kurzfristig und reversibel. Dazu zählen physiologische (Regulation der Spaltöffnungen, Blattwassergehalt) und biochemische Anpassungsreaktionen (Kätzel et al. 2008).

Nachhaltige Schäden treten auf, wenn die Belastung durch Störungen in Intensität oder Zeitdauer größer ist, als das Adaptions-, Abwehr- oder Erholungspotenzial (Kätzel et al. 2008). Mit dem Überschreiten dieses Grenzbereichs wird eine Phase der Schwächung eingeleitet. Die Anfälligkeit gegenüber weiteren Störungen ist erhöht und kann zum frühzeitigen Zusammenbruch oder zu irreversiblen (chronischen) Schädigungen führen (Lyr et al. 1992; Larcher 1994). 


\subsubsection{Indikatoren zur Messung der Wirksamkeit von Störungen}

Die starke Zeitabhängigkeit der Anpassungsprozesse stellt die Messung und Bewertung der aktuellen Störungen und deren Wirksamkeit vor große Herausforderungen. Der Vergleich der Symptome mit dem Normverhalten einer Pflanze kann Aufschluss über den aktuelle Belastungszustand geben. Diese Symptome können destruktive Vorgänge der Destabilisierung, aber auch konstruktive Vorgänge der Reparatur und Resistenzerweiterung aufzeigen. Zudem sind viele Symptome nicht reizspezifisch und können daher keinem Reiz direkt zugeordnet werden. Durch die schwankenden klimatischen Bedingungen im Freiland verlaufen Reizreaktionen oft weniger heftig als unter kontrollierten Laborbedingungen (Larcher 1994). Die Reizantwort der Pflanze auf eine kombinierte oder aufeinanderfolgende Einwirkung von Störungen kann verstärkt, abgemildert, überdeckt oder auch umgekehrt verlaufen (Burian et al. 1982).

In dieser Arbeit werden die Reaktionen der Pflanzen anhand von Indikatoren bewertet. Dabei sollen die Reaktionsmuster untersucht und Unterschiede zwischen den Baumarten und den Substraten herausgearbeitet werden. Die verwendeten Indikatoren sind im Abschnitt 2.4 „Messmethoden“ genauer beschrieben und werden im Abschnitt 4.5.2 „Indikatorenkritik“ diskutiert und bewertet.

\subsection{Versuchsmotivation}

Die Wälder in der Rhein-Main-Ebene und besonders im Hessischen Ried sind besonders exponiert gegenüber klimatischen Extremereignissen. Zusätzlich fördert die Waldzerschneidung die Schadwirkung von Stoffeinträgen aus der Luft und gefährdet das Waldinnenklima. Auf die so geschwächten Waldbestände wirken biotische Belastungen wie Massenvermehrungen von Insekten und Mistelbefall ein und führen zu einer weiteren Schwächung der Vitalität (Jacobsen und Ullrich 2010). In der Konsequenz ist in einigen Waldbereichen der Fortbestand des Hochwaldes gefährdet und ein geordneter Forstbetrieb kaum noch möglich (NW-FVA 2017). Negative Auswirkungen wie Ertragseinbußen, Störung der Betriebsstruktur, Erhöhung des betrieblichen Risikos und Einschränkungen in der Baumartenwahl prägen die forstbetrieblichen Entscheidungen und engen Handlungspielräume ein. Neben den beschriebenen Einschränkungen der Nutzfunktion werden zunehmend auch weitere Waldfunktionen gefährdet (Jacobsen und Ullrich 2010). Die Verjüngung der Waldbestände ist durch eine flächige Vergrasung unter den aufgelichteten Beständen deutlich erschwert. Die angestrebte Begründung von Mischbeständen kann meist nur durch ein Abweichen von üblichen Verjüngungsmethoden und mittels künstlicher Einbringung von Waldbäumen erfolgen.

Aus heutiger Sicht ist von einer Verstärkung der Schadprozesse auszugehen. Nur eine Sanierung der forstlichen Standorte kann zu einer grundlegenden und dauerhaften Verbesserung der gegenwärtigen Situation führen (Jacobsen und Ullrich 2010).

Um den Wald in der Region dauerhaft zu erhalten, sind langfristige ausgerichtete Anpassungsstrategien notwendig. Der in dieser Arbeit vorgestellte Versuch soll den Anwuchserfolg von Kiefernund Hainbuchenkulturen fördern und gegen Trockenphasen stabilisieren. Bei Erfolg, kann eine damit mögliche Einbringung von Laubbaumarten einen Beitrag zu einer naturnäheren Baumartenzusammensetzung leisten. Die langfristige $\mathrm{CO}_{2}$-Bindung im Boden in Form von zersetzungsstabilem Kohlenstoff kann als eine räumlich begrenzte Strategie der Mitigation gewertet werden. 


\subsection{Biokohle}

Als Vorbild für das eingesetzte Verfahren dient die „Terra preta“ in den feuchten Tropen Amazoniens, die durch Sombroek (1966) und Glaser (1999) erstmals genauer beschrieben worden ist. In diesem Boden findet sich eine Mischung aus Holz- bzw. Pflanzenkohle und weiteren Siedlungsabfällen. Vor allem die Kohle mit ihrer kohlenstoffreichen und porösen Struktur (Verheijen et al. 2010) verhilft diesem anthropogen entstandenen Boden zu seiner äußerst hohen Fruchtbarkeit. Die Wirkung von Biokohle als Bodenzusatzstoff wurde in zahlreichen Studien in unterschiedlichen Landnutzungssystemen und Standorten untersucht (Lehmann und Joseph 2009). Die Ergebnisse dieser Studien sind aufgrund der komplexen Interaktionen zwischen Biokohle, Boden und Pflanze nicht einfach zu interpretieren und zum Teil auch widersprüchlich (Lychuk et al. 2015), zumal die Eigenschaften der Biokohlen sehr stark von den Ausgangssubstraten und den Herstellungsprozessen abhängen (Verheijen et al. 2010). Biokohle entsteht durch die pyrolytische Verkohlung von organischem Ausgangsstoffen bei Temperaturen zwischen 300 und $1000{ }^{\circ} \mathrm{C}$ in einer sauerstofflimitierten Umgebung.

\subsubsection{Bodenphysikalische Wirkungen von Biokohle}

Biokohlen mit ihren sehr hohen Porenvolumina und geringen Trockenrohdichten haben das Potential, das Gesamtporenvolumen und die Trockenrohdichte eines Bodens deutlich zu verändern (Downie et al. 2009). Damit kann je nach Beigabemenge von Biokohle eine deutliche Verbesserung der Wasserhaltekapazität und der nutzbaren Feldkapazität erzielt werden (Piccolo et al. 1996; Kammann et al. 2011; Karhu et al. 2011). Hydrophobe (wasserabweisende) Eigenschaften von frischen Biokohlen, die vermutlich das Eindringen von Wasser in die inneren Poren verhindern, können die Erhöhung der Wasserhaltekapazität nach Biokohlenbeimischung in den Boden verzögern (Jeffery et al. 2015).

Die Datenlage zur sicheren Quantifizierung der Änderung bodenphysikalischer Eigenschaften ist bei kritischer Betrachtung unzureichend, daher können diesbezüglich keine belastbaren Aussagen getroffen werden. Vor allem die große Variabilität der durch unterschiedliche Ausgangsstoffe und Verkohlungsverfahren eingesetzten Kohle hinsichtlich innerer Struktur, mechanischer Stabilität und Partikelgrößen macht allgemeingültige Aussagen schwierig (Haubold-Rosar et al. 2014).

\subsubsection{Bodenchemische Wirkungen von Biokohle}

Die Einmischung von Biochar in Böden erhöht die Verfügbarkeit und beeinflusst die Aufnahme von Pflanzennährstoffen (Glaser et al. 2002; Jeffery et al. 2011). Dabei kann Biochar grundsätzlich eine direkte Nährstoffquelle sein (Atkinson et al. 2010), aufgrund der geringen Abbauraten ist aber nur ein geringer Anteil dieses Nährstoffpools pflanzenverfügbar (Chan et al. 2008; Chan und Xu 2009). Die Stabilität von Biokohlen im Boden wird als sehr hoch eingeschätzt. Schätzungen anhand von Ergebnissen aus Inkubationsexperimenten schwanken zwischen 60 Jahren (Gronwald et al. 2016) und tausenden von Jahren, sind aber stark von Ausgangsmaterial, Herstellungsbedingungen der Kohle, Boden und Klima abhängig (Lehmann et al. 2006).

Aufgrund der zahlreichen Mikroporen und der damit verbundenen großen inneren Oberfläche können Biokohlen zahlreiche mineralische und organische Makro- und Mikronährstoffe adsorbieren (Lehmann 2007). Die inneren Oberflächen von Biokohlen sind meist negativ geladen, dies erleichtert die elektrostatische Anziehung von positiv geladen organischen Kationen (Ahmad et al. 2014). Biokohlen haben pro Kohlenstoffeinheit eine größere Kationenaustauschkapazität als andere organischen Bodenmaterialien (Sombroek et al. 2003; Liang et al. 2006).

Die Zugabe von Biokohlen in den Boden kann Auswirkungen auf die Verfügbarkeit von Stick- 
stoff haben. Die so veränderte Mineralisierung (Clough und Condron 2010; DeLuca et al. 2015) und mikrobielle Umsetzung von Stickstoff (Rillig et al. 2010; Warnock et al. 2010; Lehmann et al. 2011; Spokas et al. 2012) kann positive Effekte auf Nährstoffspeicherung (Sohi et al. 2010) Biomasseerträge (Spokas et al. 2012) und Stickstoffnutzungseffizienz (Zhu et al. 2015) haben. Ausgangsmaterial, Pyrolysetemperatur, Dauer der Anheizphase, Dauer der Maximaltemperatur während des Pyrolysevorgangs, Eintragsmenge in den Boden und eventuelle Nachbehandlungen der Biokohlen haben einen starken Einfluss auf die letztendlich erzielbare Stickstoffverfügbarkeit (Gundale und DeLuca 2006; Amonette und Joseph 2009; Joseph et al. 2010). Um Stickstoffmangelerscheinungen zu vermeiden, empfehlen Chan et al. (2008) eine ergänzende angepasste Stickstoffdüngung in der landwirtschaftlichen Anwendung.

\subsubsection{Forschung zur Anwendung im forstlichen Bereich}

Die Untersuchungen zum Einsatz von Biokohle im forstlichen Bereich beschränken sich bisher auf Sonderanwendungen. So wurde durch das Verbundprojekt LaTerra unter anderem der Kohleneinsatz bei der Rekultivierung von Haldeflächen (Bodenverbesserung von Kipprohböden) im Braunkohletagebau und auf militärischen Konversionsflächen näher untersucht (Worzyk et al. 2014). In beiden Fällen wird eine nachfolgende Nutzung der Standorte als Kurzumtriebsplantage angestrebt. In einem weiteren Teilprojekt werden Weihnachtsbaumkulturen auf ehemaligen Windwurffächen im Sauerland angelegt. Die Nährstoffversorgung der Bäume soll verbessert und die Nährstoffauswaschung durch die Einarbeitung von Biokohle minimiert werden (Drabkin 2018).

Der Einsatz von Biokohle im Rahmen einer regulären Verjüngung eines Waldbestandes ist meines Wissens bisher nicht wissenschaftlich begleitet worden.

\subsection{Untersuchungsziel und Hypothesen}

In dieser Arbeit sollen die Wirkungen der Umweltfaktoren Trockenheit, Bodenbearbeitung und Stickstoffverfügbarkeit auf eine Verjüngung, bestehend aus Hainbuchen und Kiefern untersucht werden. Anhand eines Feldversuches, bei dem Biokohle in den Oberboden eingearbeitet wurde, und ergänzenden Experimenten (Topf- und Extraktionsversuch) werden die Effekte beschrieben und bewertet. Auf der Basis der folgenden Hypothesen soll dargestellt werden, wie Biokohle die Wirkungen der Umweltfaktoren bzw. Störungen im Boden und an Pflanzen modifiziert.

Durch die Biokohleeinarbeitung wird die Wasserhaltekapazität des Oberbodens wesentlich erhöht. Die erforderliche Kohlemenge ist so bemessen, dass eine Erhöhung der pflanzennutzbaren Feldkapazität um $15 \%$ erreicht wird (1).

Durch die chemische und biologische Nachbehandlung der Kohlen werden die chemischen Eigenschaften des Oberbodens nach Kohleneinarbeitung deutlich verändert $(2)$.

Die Pflanzen auf den Parzellen der Biokohlevarianten weisen höhere Überlebenszahlen als die Pflanzen der Nullvariante auf (3). 


\section{Material und Methoden}

\subsection{Standort}

\subsubsection{Lage, Relief, Exposition}

Die Versuchsfläche liegt in der Rhein-Mainebene im Hessischen Ried, Forstamt Lampertheim, in Abteilung 203 des Reviers Viernheim (siehe Abb. 2.1). Südlich angrenzend verläuft die Landesgrenze zu Baden-Württemberg. Hier geht der Viernheimer Wald nahtlos in den Käfertaler Wald der Stadt Mannheim über.

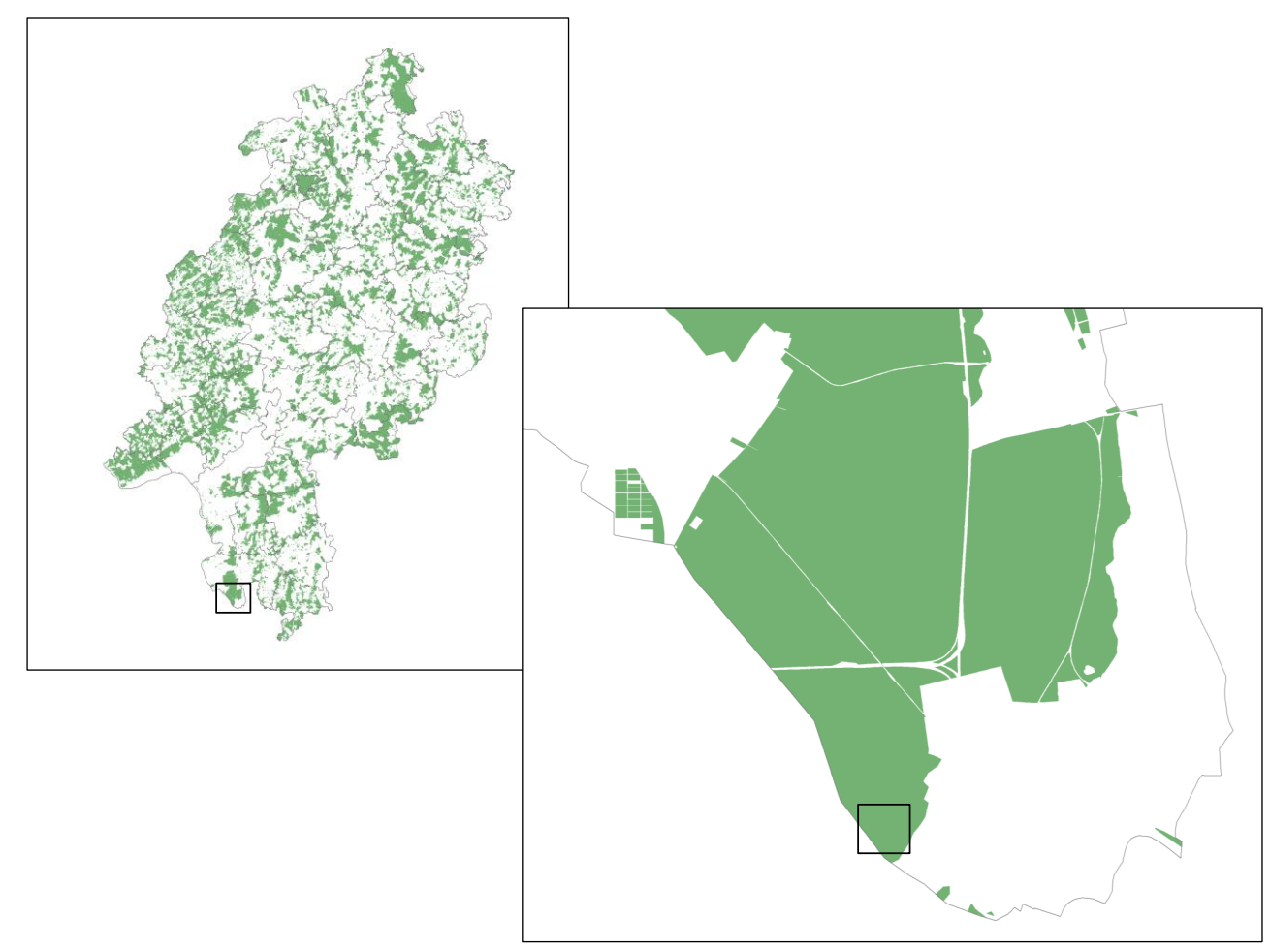

Abbildung 2.1: Lage der Versuchsfläche in Südhessen

Das Relief ist auf der Versuchsfläche und im angrenzenden Waldgebiet durch den Wechsel von dünenartigen Hügeln und ebenen Arealen geprägt (siehe Abb. 2.2). Die Höhendifferenz zwischen Dünenrücken und Ebene beträgt etwa drei bis vier Meter. Die Entstehung dieser Strukturen ist auf eine späte Phase von Sandverwehungen in einer regional weitgehend waldfreien Zeit des späten Mittelalters zurückzuführen (siehe Abschnitt „kalkhaltige Sande“ auf Seite 27). Die Dünenhänge auf der Versuchsfläche sind hauptsächlich nach Süden und Norden ausgerichtet. 


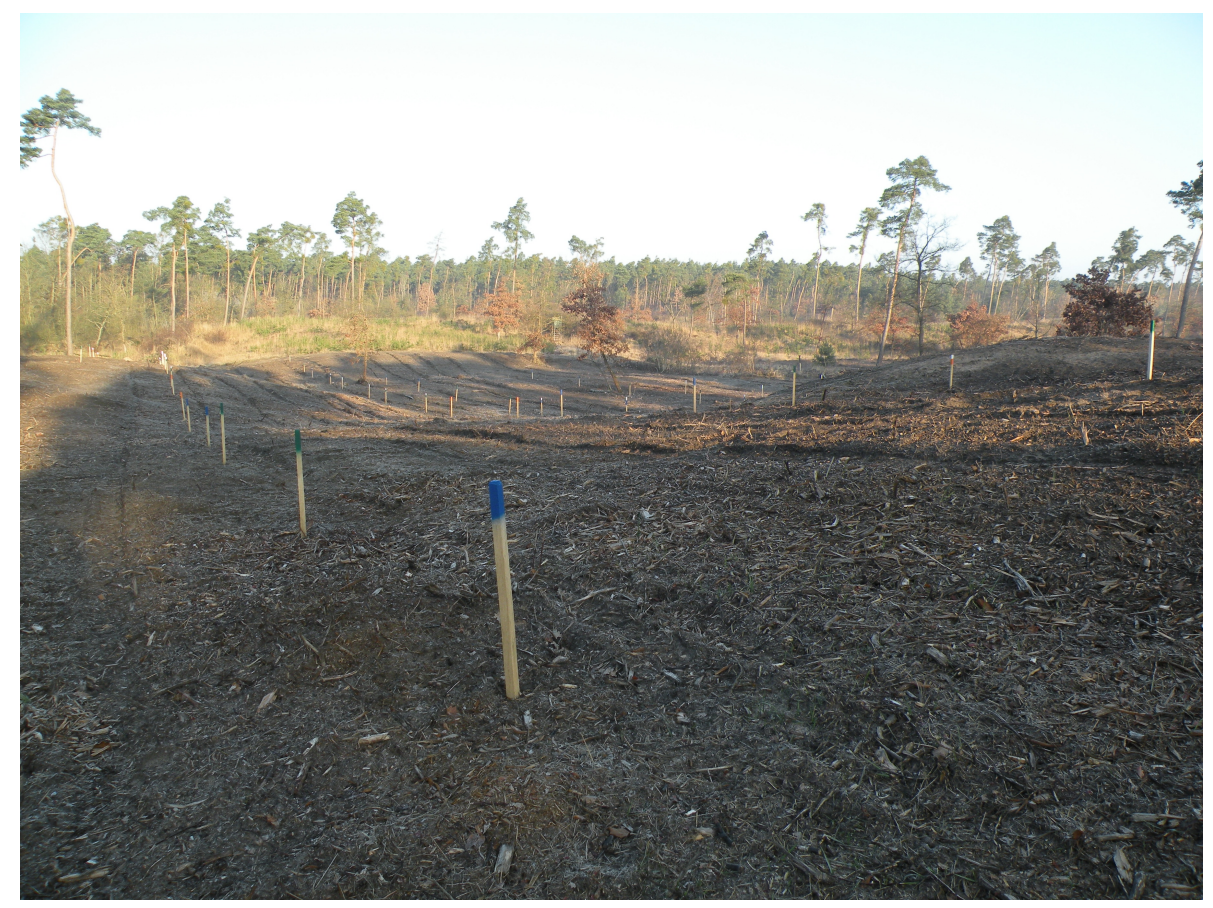

Abbildung 2.2: Relief der Versuchsfläche

\subsubsection{Klima und Witterung}

Als Klima wird der mittlere Zustand der Atmosphäre bezeichnet, der über viele Jahre an einem Ort beobachtet und gemessen wurde (Sitte et al. 2002; Standortskartierung 2003).

Die Versuchsfläche liegt in einer Höhenlage von etwa $100 \mathrm{~m}$ über NN. Das Klima ist schwach subkontinental, durch hohe Durchschnittstemperaturen während der Vegetationszeit $\left(16{ }^{\circ} \mathrm{C}\right)$ und relativ geringe Jahresniederschläge $(670 \mathrm{~mm})$ gekennzeichnet. Aufgrund des warmen Klimas und der monatlichen Temperatursummen wird für die Vegetationsperiode in dieser Arbeit abweichend von der Forstlichen Standortskartierung (Mai - September) der Zeitraum 1. April - 31. Oktober definiert (Standortskartierung 2003).

Die in etwa $5 \mathrm{~km}$ Luftlinie entfernte DWD-Klimastation Mannheim liefert meteorologische Daten in täglicher Auflösung wie Maximal- und Minimaltemperatur, mittlere Temperatur, Niederschlagssumme, Sonnenscheindauer, mittlere Windgeschwindigkeit und die mittlere relative Luftfeuchte.

Der Zeitraum um die Versuchseinrichtung im März und April 2014 war deutlich wärmer und trockener als im langjährigen Mittel (siehe Tabellen 2.1, 2.2 und Abbildung A.1 im Anhang). Bereits die vorangegangene Nichtvegetationszeit war um $20 \%$ trockener als das langjährige Mittel. Im März, Mai und Juni fielen weniger als $50 \%$ des mittleren Niederschlages. Erst in den Folgemonaten Juli und August waren bis zu 50 Prozent höhere Niederschlagssummen zu verzeichnen, so dass insgesamt eine ausreichende Wasserversorgung während der Vegetationszeit sichergestellt war. 
Tabelle 2.1: Mittlere Monatstemperaturen in ${ }^{\circ} \mathrm{C}$ der Jahre 2013 - 2017 und des langjährigen Mittels 1961 - 1990 (Deutscher Wetterdienst, Station Mannheim, 2018)

\begin{tabular}{ccccccccccccc}
\hline & \multirow{2}{*}{ Jan } & Feb & März & Apr & Mai & Jun & Jul & Aug & Sep & Okt & Nov & Dez \\
\hline $1961-1990$ & 1,2 & 2,5 & 5,9 & 9,9 & 14,4 & 17,6 & 19,5 & 18,8 & 15,3 & 10,4 & 5,2 & 2,2 \\
2013 & 2,8 & 1,6 & 4,0 & 11,2 & 13,4 & 18,4 & 22,9 & 20,3 & 15,9 & 12,6 & 6,4 & 5,0 \\
2014 & 4,6 & 5,8 & 8,8 & 13,3 & 14,4 & 18,9 & 20,9 & 17,8 & 16,5 & 13,4 & 7,6 & 4,3 \\
2015 & 3,1 & 2,0 & 7,0 & 11,0 & 15,4 & 18,8 & 22,8 & 22,0 & 15,0 & 10,1 & 8,4 & 7,4 \\
2016 & 4,0 & 5,3 & 6,0 & 9,8 & 15,2 & 18,4 & 21,1 & 20,3 & 18,6 & 9,8 & 5,6 & 2,3 \\
2017 & $-0,6$ & 6,1 & 10,1 & 10,7 & 17,1 & 21,7 & 21,8 & 20,9 & 15,1 & 12,7 & 6,7 & 4,4 \\
\hline
\end{tabular}

Während der Vegetationszeit 2015 trat in den mittleren Regionen Deutschlands eine außergewöhnliche Trockenperiode auf, die in Südhessen bis in den Spätsommer anhielt (Sutmöller et al. 2017). Von April bis Juli erreichte das Niederschlagsdefizit im Rhein-Main-Gebiet mehr als $50 \%$. In dieser Vegetationszeit wurden auf den Level II-Flächen Tiefstwerte bei den Bodenwassergehalten gemessen (Sutmöller et al. 2017). Auch im Untersuchungsgebiet traten während der Vegetationszeit 2015 unterdurchschnittliche Niederschlagssummen und mehrere Trockenperioden auf. Wie etwa vom 1. bis 18. Juni und vom 21. Juni bis 7. Juli.

Die durchschnittlichen Monatswerte von Temperatur und Niederschlag zeigen im Jahr 2016 eine deutlich ausgeglichenere Witterung. Der erste Teil der Vegetationszeit war von regelmäßigen Niederschlägen begleitet. Erst in der zweiten Augusthälfte trat eine Trockenperiode auf, die bis Mitte September anhielt.

In den Monaten Dezember 2016 bis April 2017 waren unterdurchschnittliche monatliche Niederschlagssummen zu verzeichnen. Die Monate Januar und April des Jahres 2017 waren dabei deutlich zu trocken mit Niederschlagssummen von 20 bzw. $7 \mathrm{~mm}$. Die anschließende Vegetationsperiode 2017 zeigte dagegen regelmäßige Niederschlagsereignisse, aber zwischen 2 bis $4{ }^{\circ} \mathrm{C}$ höhere Monatsmitteltemperaturen als im Referenzzeitraum 1961 - 1990.

Tabelle 2.2: Monatliche Niederschlagssummen in mm der Jahre 2013 - 2017 und des langjährigen Mittels 1961 - 1990 (Deutscher Wetterdienst, Station Mannheim, 2018).

\begin{tabular}{ccccccccccccc}
\hline & \multirow{2}{*}{ Jan } & Feb & März & Apr & Mai & Jun & Jul & Aug & Sep & Okt & Nov & Dez \\
\hline $1961-1990$ & 40 & 40 & 45 & 52 & 75 & 77 & 77 & 59 & 54 & 49 & 52 & 49 \\
2013 & 31 & 41 & 31 & 53 & 156 & 110 & 14 & 47 & 79 & 96 & 55 & 47 \\
2014 & 35 & 39 & 10 & 50 & 46 & 35 & 126 & 86 & 40 & 49 & 50 & 69 \\
2015 & 86 & 22 & 21 & 20 & 40 & 65 & 39 & 52 & 46 & 16 & 77 & 22 \\
2016 & 60 & 59 & 45 & 64 & 100 & 94 & 52 & 43 & 25 & 75 & 57 & 8 \\
2017 & 20 & 27 & 47 & 7 & 80 & 61 & 78 & 67 & 62 & 44 & 77 & 89 \\
\hline
\end{tabular}




\subsubsection{Ausgangszustand des Bodens}

In diesem Abschnitt werden bereits einige selbsterhobene Ergebnisse präsentiert. Da sie in erster Linie der Charakterisierung des Standortes vor Versuchsanlage dienen, werden sie bereits hier im Material und Methodenteil vorgestellt, um die Lesbarkeit der Arbeit zu verbessern.

Die Beprobung mittels Pirckhauer-Stichproben in mehreren Transektlinien bis in $1 \mathrm{~m}$ Bodentiefe ergab einen homogenen, tiefgründigen Sandstandort aus pleistozänen Flugsandens. Als Bodentyp des Leitprofils wurde eine Braunerde aus unverlehmten Sanden ausgewiesen. Vor dem Fräsen der gesamten Versuchsfläche wurde eine feinhumusreiche, rohhumusartige Moder-Humusform angesprochen. Die Standortskartierung weist für diese Fläche einen mäßig frischen Geländewasserhaushalt und eine mesotrophe Nährstoffversorgung aus (HessenForst 2014).

\subsubsection{Korngrößenverteilung}

Die Korngrößenverteilung gibt Aufschluss über die Massenanteile der unterschiedlichen Kornfraktionen eines Bodens.

Die Probenahme für die Korngrößenbestimmung wurde zeitgleich mit der Probennahme für die Retentionskurvenermittlung (pF-Kurven) durchgeführt. Dafür wurden jeweils etwa $300 \mathrm{~g}$ Bodenmaterial aus unmittelbarer Nähe der pF-Stechzylinderproben entnommen. Auf jeweils einer Parzelle der Behandlungen Null, HKpur, SKpur und HK+N+Ko wurden in zwei Tiefenstufen $(0-30 \mathrm{~cm}$ und $30-60 \mathrm{~cm})$ jeweils drei Proben entnommen.

Die Proben wurden nach der Vorbereitung in Göttingen (Trocknung und Siebung) durch das Landesamt für Geologie und Bergbau Rheinland-Pfalz in Mainz analysiert. Dabei kam das Verfahren der Schlämmkornanalyse - Pipettmethode zur Anwendung, welches auf der Grundlage unterschiedlicher Sedimentationsgeschwindigkeiten beruht (Moschrefi 1983). Dabei wird die Bodensuspension in einen Sedimentationszylinder eingeschlämmt. Durch das Sieben auf $2 \mathrm{~mm}$ und die Einschlämmung besteht die Gefahr der Reduktion des Biokohlenanteils in den Bodenproben, da die ausgesiebten bzw. oben aufschwimmenden Bestandteile nicht analysiert werden. Dieser Anteil war jedoch laut Aussage des Laborleiters sehr gering.

Tabelle 2.3: Korngrößenverteilung auf einer Parzelle der Nullvariante. Angaben in \%. $\mathrm{fU}=$ Feinschluff, $\mathrm{mU}=$ Mittelschluff, $\mathrm{gU}=$ Grobschluff, $\mathrm{fS}=$ Feinsand, $\mathrm{mS}=$ Mittelsand, $\mathrm{gS}=$ Grobsand

\begin{tabular}{ccccccccc}
\hline Bodentiefe & Ton & $\mathrm{fU}$ & $\mathrm{mU}$ & $\mathrm{gU}$ & $\mathrm{fS}$ & $\mathrm{mS}$ & $\mathrm{gS}$ & Bodenart \\
\hline $0-30 \mathrm{~cm}$ & 3,9 & 0,5 & 1,3 & 1,1 & 32,3 & 59,5 & 1,4 & $\mathrm{mSfs}$ \\
$30-60 \mathrm{~cm}$ & 2,5 & 0,0 & 0,2 & 0,7 & 33,3 & 62,3 & 1,0 & $\mathrm{mSfs}$ \\
\hline
\end{tabular}

Die Korngrößenverteilung des Ausgangsbodens (Nullvariante) wird durch überwiegend sandige Fraktionen ohne Grobbodenanteile bestimmt (siehe Tab. 2.3). Die resultierende Bodenart ist daher ein feinsandiger Mittelsand (mSfs).

\subsubsection{Retentionsskurven (pF-Kurven)}

Der Verlauf der Kurve zwischen Wassergehalt und Matrixpotential bzw. Wasserspannung ist für jede Bodenart charakteristisch und ist von Porenvolumen und Porengrößenverteilung abhängig. Die Kurve ist Basis für die Berechnung von Wasserbewegungen und anderen Größen des Wasserhaushaltes (Scheffer 2002). Der pF-Wert ist der Logarithmus der Wasserspannung.

Die Wasserspannungskurve wurde per Druckmethode nach L. A. Richards bestimmt (Richards 1948). Die wassergesättigte Bodenprobe wird auf einer keramischen Platte mit Luftdrücken, die 
bestimmten Wasserspannungen entsprechen, ins Gleichgewicht gebracht. Die aus dem Boden verdrängte Wassermenge wird gemessen. Die Wasserspannungskurve erhält man, wenn die angewandten Drücke gegen die jeweiligen Wassergehalte aufgetragen werden (Scheffer 2002).

Die Analysen wurden in der Abteilung für Ökopedologie der gemäßigten Zonen am BüsgenInstitut der Fakultät für Forstwissenschaften und Waldökologie Göttingen durchgeführt. Es wurden Bodenproben aus den Bodentiefen $0-10 \mathrm{~cm}, 10-20 \mathrm{~cm}, 20-30 \mathrm{~cm}, 30-40 \mathrm{~cm}$ und $50-60$ $\mathrm{cm}$ für die Ermittlung der Wasserspannungskurven herangezogen. Für den Oberboden sind die Werte der beiden oberen Horizonte $(0-20 \mathrm{~cm})$, für den Unterboden die Werte der zwei unteren Horizonte $(30-60 \mathrm{~cm})$ zusammengefasst worden.

Die Wasserspannungskurve des Substrates der Nullvariante zeigt die sandige Struktur sowohl des Ober- als auch des Unterbodens (siehe Abb. 2.3).
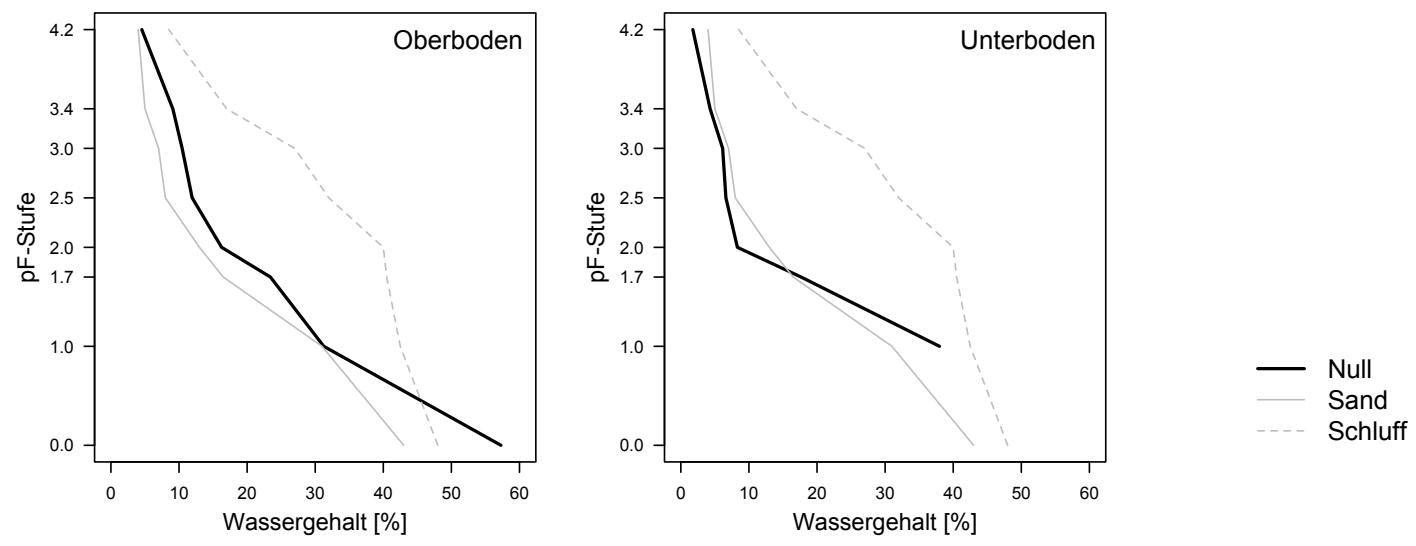

Abbildung 2.3: Retentionskurven (pF-Wassergehalt) des Oberboden (0 - $20 \mathrm{~cm}$, links) und des Unterbodens (30 - $60 \mathrm{~cm}$, rechts) der Nullvariante (schwarze Linie). Werte für Korngrößengruppen Sand (grau durchgezogene Linie) und Schluff (grau gestrichelte Linie) aus Hartge et al. 2014

Der Permanente Welkepunkt (PWP) ist der Wassergehalt, bei dem die Pflanzen irreversibel welken. Die Feldkapazität (FK) ist der Wassergehalt, den ein Boden maximal gegen die Schwerkraft zurückhalten kann. Die nutzbare Feldkapazität (nFK) ist ein Maß für die Menge an pflanzenverfügbaren Bodenwasser und eine wichtige Größe zur Beschreibung des Wasserhaushaltes. Sie berechnet sich aus der Differenz des Bodenwassergehaltes zwischen Feldkapazität (pF 1,8) und Permanentem Welkepunkt ( $\mathrm{pF} 4,2$ ) und repräsentiert die Menge des Bodenwassers, das in Poren des Durchmessers zwischen 50 und 0,2 $\mu \mathrm{m}$ gebunden ist (AG-Boden 2005).

Die nutzbare Feldkapazität beträgt 18,9 Vol.\% (mm) je $1 \mathrm{dm}$ Bodentiefe im Oberboden und 18,8 Vol.\% (mm) pro $1 \mathrm{dm}$ Bodentiefe im Unterboden. Die Berechnung der Summe der nutzbaren Feldkapazität bis auf $90 \mathrm{~cm}$ geschieht durch das Aufsummieren der Einzel-nFK-Beträge pro Tiefenstufe. Damit ergibt sich rechnerisch eine nutzbare Feldkapazität von 148,4 mm. Die Berechnung durch die Feldmethode gemäß der Forstlichen Standortsaufnahme (Standortskartierung 2003) ergibt knapp $140 \mathrm{~mm}$ nutzbare Feldkapazität und damit die Einstufung in die frische Geländewasserhaushaltsstufe nach hessischer Standortskartierung. Dies steht im Widerspruch zur 
mäßig frischen Geländewasserhaushaltsstufe, die durch Standortskartierung für diesen Bereich ausgewisen wurde.

\subsubsection{Trockenrohdichte}

Die Trockenrohdichte gibt Auskunft über die Verfestigung des Bodens und über das Gesamtporenvolumen eines Bodens. Als Trockengewicht pro Volumeneinheit ist sie Voraussetzung für Umrechnungen einer gewichtsbasierten Größe in eine volumenbasierte Größe (Standortskartierung 2003).

Die Trockenrohdichte wurde mittels der Stechzylindermethode nach Klute (1986) im Januar 2014, und jeweils im April 2015 und 2016 ermittelt. Die volumengerechten Stechzylinderproben wurden auf Parzellen der Nullvariante $(1 / 7)$ und verschiedener Kohlevarianten (5/1, 4/4 und $3 / 3$ ) gewonnen. Folgende Tiefenstufen wurden beprobt: 0 - $10 \mathrm{~cm}, 10-20 \mathrm{~cm}, 20-30 \mathrm{~cm}, 30$ - $60 \mathrm{~cm}$ und 60 - $90 \mathrm{~cm}$, jeweils in dreifacher Wiederholung. Aufgrund des skelettfreien Bodens konnte ein $250 \mathrm{ml}$ Stechzylinder verwendet werden. Die Proben wurden in Leinensäcke gefüllt, beschriftet, getrocknet und anschließend gewogen. Die Trockenrohdichte ergibt sich aus dem Gewicht der Bodenfestsubstanz pro Volumeneinheit nach Trocknung bei $105^{\circ} \mathrm{C}$ für mindestens 16 Stunden (Standortskartierung 2003).

Die Trockenrohdichte des Feinbodens $\left(\mathrm{TRD}_{\mathrm{FB}}\right)$ wird nach folgender Formel berechnet:

$$
T R D_{F B}=\frac{M_{g e s} S Z}{V_{g e s} S Z}
$$

Der Feinbodenvorrat (FBV) kann nach folgender Formel berechnet werden:

mit

$$
\mathrm{FBV}=\mathrm{TRD}_{\mathrm{FB}} * \mathrm{~d} * 100
$$

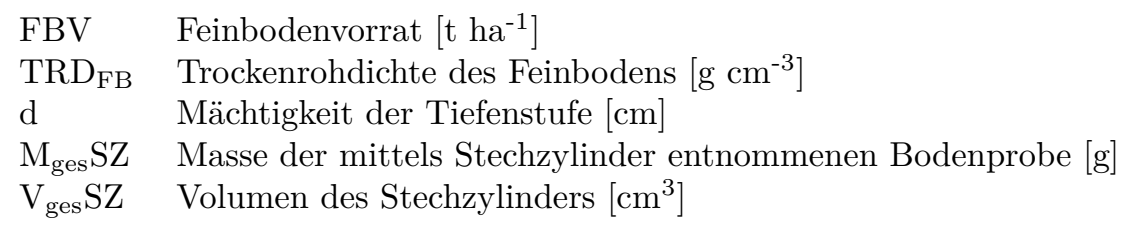

Die Trockenrohdichten des Ausgangsbodens (Nullvariante) zeigen deutlich die Bodenbearbeitung im Zuge der Kohleneinarbeitung im Jahr 2014 in den Oberboden. Die Nullflächen wurden ebenfalls bis etwa $25 \mathrm{~cm}$ gefräst, um eine vergleichbare Ausgangssituation zu schaffen.

Tabelle 2.4: Mittlere Trockenrohdichten in $\mathrm{g} \mathrm{cm}^{-3}$ des Ausgangsbodens (Nullvariante) im Jahr 2014 (nach Bodenbearbeitung), $\mathrm{n}=3$.

\begin{tabular}{cc}
\hline Bodentiefe & 2014 \\
\hline $0-10 \mathrm{~cm}$ & 0,996 \\
$10-20 \mathrm{~cm}$ & 1,313 \\
$20-30 \mathrm{~cm}$ & 1,372 \\
$30-60 \mathrm{~cm}$ & 1,622 \\
$60-90 \mathrm{~cm}$ & 1,553 \\
\hline
\end{tabular}




\subsubsection{Bodenprobenahme und chemische Analysen}

An der westlichen Grenze des Versuchsfeldes wurde Ende 2013 ein Baggerprofil angelegt und beprobt. Von den Nullparzellen wurden Anfang 2014 Proben gezogen. Im November 2015 und im Dezember 2016 wurden alle Versuchsparzellen beprobt. Dazu wurde aus drei Pirckhauerproben eine Mischprobe pro Parzelle und Tiefenstufe hergestellt. Auf allen Flächen wurden die Tiefenstufen 0 - $30 \mathrm{~cm}$ und 30 - $60 \mathrm{~cm}$ beprobt, auf jeweils einer Parzelle je Versuchsvariante zusätzlich die Tiefenstufe 60 - $90 \mathrm{~cm}$. Die Proben wurden feldfrisch in Leinenbeuteln transportiert und im Labor bei $40^{\circ} \mathrm{C}$ getrocknet, bevor sie gemahlen und chemisch analysiert wurden (König 2009). Durch das kalkhaltige Ausgangssubstrat (siehe Abschnitt „kalkhaltige Sande“ auf Seite 27) zeigen vor allem die Bodenproben des Unterbodens einen pH-Wert deutlich über 6,2, daher wurde 2014 an diesen Proben die potentielle Austauschkapazität $\left(\mathrm{AK}_{\mathrm{t}}\right.$ ) bestimmt (siehe Abb. 2.4). Die Bestimmung der effektiven Austauschkapazität wurde 2014 und 2015 an Bodenproben durchgeführt, die einen pH-Wert unter 6,2 aufwiesen.
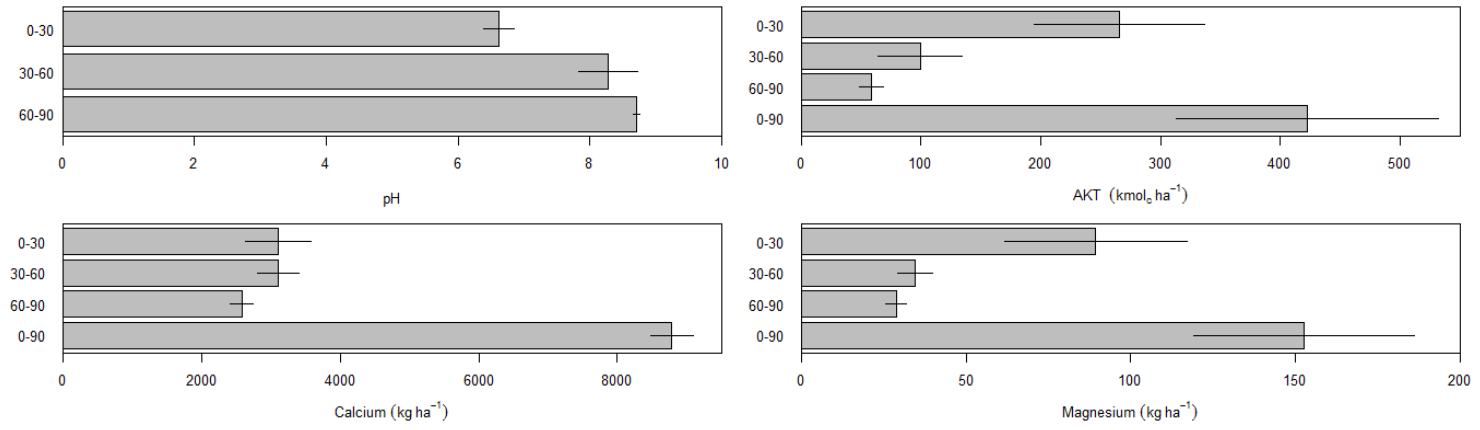

Abbildung 2.4: pH-Wert, potenzielle Austauschkapazität (AKt) [ $\left.\mathrm{kmol}_{\mathrm{c}} \mathrm{ha}^{-1}\right]$, Calcium- und Magnesiumvorrat $\left[\mathrm{kg} \mathrm{ha}{ }^{-1}\right]$ der Nullflächen $(\mathrm{n}=5)$ vor Versuchsanlage (Mittelwert und Standardabweichung).
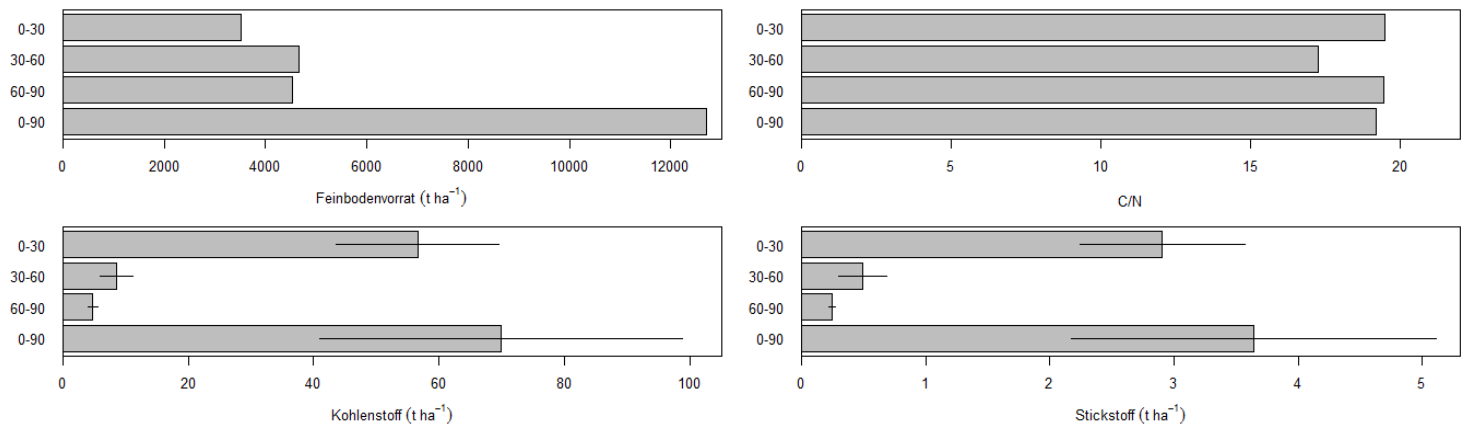

Abbildung 2.5: Feinbodenvorrat $\left[\mathrm{t} \quad \mathrm{ha}^{-1}\right]$, C/N-Verhältnis, Kohlenstoff- und Stickstoffvorrat $\left[\mathrm{t} \mathrm{ha}^{-1}\right]$ der Nullflächen $(\mathrm{n}=5)$ vor Versuchsanlage (Mittelwert und Standardabweichung).

In den Abbildungen 2.4 bis 2.5 und der Tabelle A.2 (im Anhang) sind die Ergebnisse der Bodenchemie im Überblick dargestellt. Sie fassen den Bodenzustand nach dem Mulchen und vor 
der Einarbeitung der Kohle in den Oberboden zusammen. Weil Parzelle 1/7 in einem sauren Senkenbereich liegt (siehe Abschnitt „kalkhaltige Sande“ auf Seite 27) sind die Ergebnisse der Parzelle 1/7 in Tabelle A.2 gesondert aufgeführt und für die Berechnung der mittleren Vorräte (siehe Abb. 2.4 bis 2.5) nicht berücksichtigt worden.

Der Feinbodenvorrat bis in $90 \mathrm{~cm}$ Bodentiefe ist mit $12705 \mathrm{t} \mathrm{ha}^{-1}$ typisch für die Substratgruppe der unverlehmten Sande als Substratgruppe und kann durch die hohen Trockenrohdichten und geringen Grobbodenanteile erklärt werden (Paar et al. 2016).

Die pH-Werte der Nullparzellen liegen mit Ausnahme der Parzelle 1/7 deutlich über 6,2. Damit liegt der Boden im Carbonatpufferbereich nach (Ulrich 1981). Die potentielle Kationenaustauschkapazität ist eine wichtige Indikatorgröße für die standörtliche Bewertung des Nährstoffhaushaltes, mit der Speicher- und Austauschprozesse von pflanzenverfügbaren Nährstoffen an den Tonmineraloberflächen beschrieben werden können (Standortskartierung 2003). Der mittlere Wert der potentiellen Austauschkapazität des Standortes vor Kohleneinarbeitung beträgt 423 $\mathrm{kmol}_{\mathrm{c}} \mathrm{ha}^{-1}$ und liegt damit im mittleren Bereich nach dem Bewertungsrahmen der Forstlichen Standortsaufnahme (Standortskartierung 2003) und ist für die Substratgruppe der unverlehmten Sande verhältnismäßig hoch (Paar et al. 2016). Die erhöhte Austauschkapazität im Oberboden kann mit dem hohen Anteil eingefräster organischer Substanz aus der ehemaligen mächtigen Humusschicht in Zusammenhang gebracht werden. Die meisten unverlehmten Sande in Hessen weisen $\mathrm{pH}$-Werte von unter 6,2 auf, für diese wird die effektive Austauschkapazität angegeben. Eine Vergleichbarkeit zwischen potenzieller und effektiver Austauschkapazität ist nicht gegeben. Die austauschbaren Vorräte an Calcium und Magnesium bilden eine wichtige Versorgungsgrundlage für die Waldbäume.

Calcium ist an zentralen Prozessen wie Photosynthese, Atmung und Zellteilung beteiligt und ein wichtiger Baustein für die Zellwände. Die mittleren Calciumvorräte bis in $90 \mathrm{~cm}$ Bodentiefe betragen $8789 \mathrm{~kg} \mathrm{ha}^{-1}$ und liegen damit im sehr hohen Bereich des Bewertungsrahmens der Forstlichen Standortsaufnahme (Standortskartierung 2003). Es lässt sich kein Tiefengradient des Calciumvorrates beobachten. Auf die Besonderheit dieses sehr hohen Calciumvorrates bei unverlehmten Sanden wird im Abschnitt „kalkhaltige Sande“ auf Seite 27 genauer eingegangen.

Magnesium ist ein zentraler Chlorophyllbestandteil und damit bedeutend für die Photosynthese. Der mittlere Magnesiumvorrat von $153 \mathrm{~kg} \mathrm{ha}^{-1}$ liegt im gering-mittleren Bereich der Forstlichen Standortsaufnahme (Standortskartierung 2003) und ist etwa doppelt so hoch wie die typischen Vorräte der unverlehmten Sande in Hessen. Wie von Paar et al. (2016) für ärmere Standorte beschrieben, lassen sich die höchsten Magnesiumvorräte in den oberen Tiefenstufen finden.

Der mittlere Kohlenstoffvorrat des organisch gebundenen Kohlenstoffes beträgt 70 t ha ${ }^{-1}$ und liegt damit im geringen Bewertungsbereich der Forstlichen Standortsaufnahme (Standortskartierung 2003). Vor allem in den oberen Bodenschichten $(0-30 \mathrm{~cm})$ liegen über $80 \%$ des Kohlenstoffes vor. Dies kann durch den untergemulchten Auflagehumus erklärt werden. Für die Substratgruppe der unverlehmten Sande ist der Kohlenstoffvorrat relativ gering, typisch sind Vorräte um $100 \mathrm{t}$ ha $^{-1}$.

Der mittlere Stickstoffvorrat auf dem Versuchsfeld vor Versuchsanlage beträgt $3,6 \mathrm{t} \mathrm{ha}^{-1}$ und liegt damit im geringen Bewertungsbereich der Forstlichen Standortsaufnahme (Standortskartierung 2003). Die Substratgruppe der unverlehmten Sande weist in Hessen typischerweise mit etwa 6,4 t ha ${ }^{-1}$ höhere Vorratswerte an Stickstoff auf. Substratgruppen mit schlechteren Humusformen zeigen die höchsten Kohlenstoff- und Stickstoffvorräte (Paar et al. 2016). Analog zu Kohlenstoff zeigt sich in den oberen Bodenschichten mit etwa 80 \% der höchste Anteil der Stickstoffvorräte. Das C/N-Verhältnis als Quotient aus den Kohlenstoff- und Stickstoffvorräten weist auf die Bedingungen für die Umsetzung von organischer Substanz hin. Je enger (kleiner) das Verhältnis, umso höher sind die Raten der Humusmineralisation und - umsetzung. Mit einem Wert von 19,5 im Bereich 0 - $30 \mathrm{~cm}$ liegt das C/N-Verhältnis im mittleren Bewertungsbereich der Forstli- 
chen Standortsaufnahme (Standortskartierung 2003) und ist typisch für die Substratgruppe der unverlehmten Sande (Paar et al. 2016).

\begin{abstract}
kalkhaltige Sande
Die Auswertung der Ende 2013, Ende 2015 und Ende 2016 gewonnen Bodenproben ergab unerwartet hohe Carbonatgehalte und pH-Werte über 6,2 bis in eine Bodentiefe von $90 \mathrm{~cm}$ auf fast allen Parzellen. Der Boden befindet sich damit im Carbonatpufferbereich (Ulrich 1981). Ausnahme ist der Senkenbereich mit den Parzellen, 1/5, 1/6, 1/7 und 2/3 (siehe Abb. 2.7).

Die Entstehungszeit der Dünen lässt sich nur ungefähr in den Zeitraum zwischen der Zeitenwende und dem Mittelalter datieren (Plass 1981). Durch windbürtige (äolische) Abtragung bzw. Umlagerung von bereits verwitterten Sandoberflächen wurden noch unverwitterte kalkhaltige Substrate freigelegt. Durch die vergleichsweise geringen Niederschläge in der Rhein-Main-Ebene verläuft die Entkalkung nur langsam und die Sande sind bis heute mit einem nennenswerten Kalkvorrat ausgestattet. Die windbürtige Umlagerung mit Korngrößenfraktionierung kann anhand der erkennbaren Bänderung mit gröberen Sanden am Bodenprofil nachvollzogen werden (siehe Abb. 2.6).
\end{abstract}

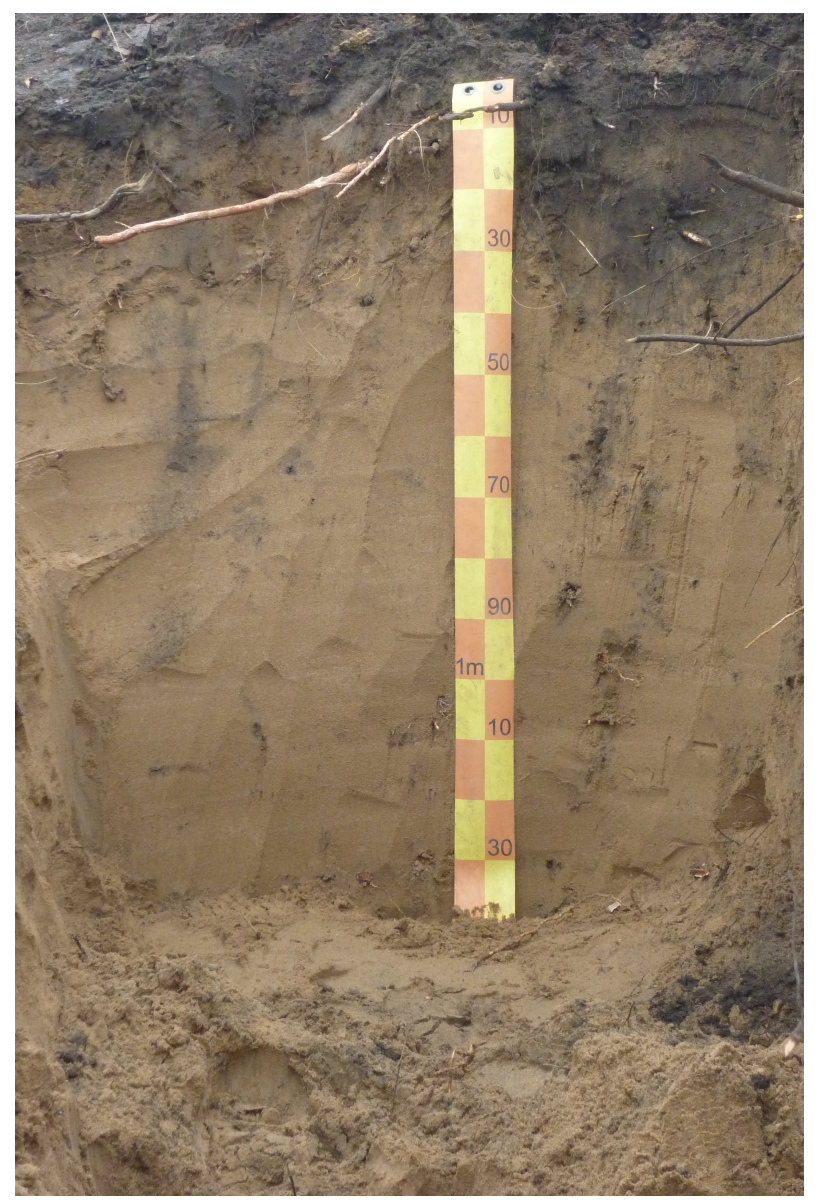

Abbildung 2.6: Das Bodenprofil auf der Versuchsfläche 


\subsection{Kohlenherstellung}

\subsubsection{Ausgangssubstrate}

Die eingesetzten Biokohlen wurden von zwei Herstellern bezogen. Als Ausgangssubstrat für die Holzkohle-Varianten wurden vom Hersteller „Pyreg“ Hackschnitzel der Spätblühenden Traubenkirsche und ein kleiner Teil Buche verwendet.

Die Spelzenkohle-Varianten sollten ursprünglich mit Maisstroh als Ausgangssubstrat hergestellt werden. Das Zerkleinern des Maisstrohs produzierte aber eine große Menge Staub und führte zu einem unerwünscht hohen Ascheanteil in der Kohle. Daraufhin wurde diese Kohlevariante vom Hersteller „Sonnenerde“ aus $66 \%$ Dinkel-Getreidespelzen und $33 \%$ Papierfaserschlamm produziert. Die Reaktionstemperatur während der Pyrolyse lag bei $600-650{ }^{\circ} \mathrm{C}$.

Tabelle 2.5: Bezeichnung der Versuchsvarianten

\begin{tabular}{ccc}
\hline Varianten & \multicolumn{2}{c}{ Ausgangssubstrate } \\
& Holz & Getreidespelzen \\
\hline Kohle pur (unbehandelt) & HKpur & SKpur \\
Kohle und Nährstofflösung & $\mathrm{HK}+\mathrm{N}$ & $\mathrm{SK}+\mathrm{N}$ \\
Kohle und Nährstofflösung und Kompostierung & $\mathrm{HK}+\mathrm{N}+\mathrm{Ko}$ & $\mathrm{SK}+\mathrm{N}+\mathrm{Ko}$ \\
Kontrolle & \multicolumn{2}{c}{ Null } \\
\hline
\end{tabular}

\subsubsection{Nachbehandlung der Kohlen}

Die Kohlevarianten N und N+Ko beider Ausgangssubstrate (siehe Tab. 2.5) wurden direkt nach der Pyrolyse mit einer Ammoniumsulfatlösung (5\%( $\left.\left.\mathrm{NH}_{4}\right)_{2} \mathrm{SO}_{4}\right)$ eingesprüht. Die Austauscherplätze der Kohle werden damit vor Ausbringung mit Stickstoff besetzt, um einen Stickstoffmangel in der Startphase zu vermeiden (Chan et al. 2008). Zusätzlich wurde Phosphorsäure (75 \%) aufgebracht, um die Alkalinität der Kohle zu reduzierenund die innere Oberfläche der Kohlen zu vergrößern.

Die von den Herstellern durchgeführten Nachbehandlungen wurden durch das Labor der NWFVA analytisch überprüft. Die angestrebte verfügbare Menge an Stickstoff und Phosphor wird abgeleitet aus den extrahierbaren Nährstoffgehalten von mitkompostierter Holzkohle (Kammann et al., unveröff.) Diese Gehalte betragen etwa 2200 mg Sticktoff und 52 mg Phophor pro kg Kohle. Die unbehandelten Kohle weisen nur etwa $10 \mathrm{mg}$ bzw. $23 \mathrm{mg}$ extrahierbaren Stickstoffes bzw. Phosphor pro kg auf.

Die Kompostvarianten der Kohlen $(\mathrm{N}+\mathrm{Ko})$ wurden für zwei Wochen in eine Bio-Kompostmiete eingemischt, mehrmals umgesetzt und anschließend auf $15 \mathrm{~mm}$ gesiebt. Durch diesen „Alterungsprozess" wird die Oberfläche der Kohle mit einer humusaufbauenden Mikrobiologie aufgeladen bzw. biologisch aktiviert. Es hat sich gezeigt, dass derart behandelte Kohlen von der Bodenbiologie wesentlich besser angenommen werden (Prost et al. 2013). 


\subsubsection{Physikalische und chemische Eigenschaften der Biokohlen im Versuch}

Die physikalischen Eigenschaften der puren Kohlen sind in Tabelle 2.6 aufgelistet. Die Spelzenkohlevariante (SK) weist eine deutlich niedrigere Trockenrohdichte und einen geringeren Wassergehalt zum Zeitpunkt der Einarbeitung in den Boden auf.

Tabelle 2.6: Physikalische Eigenschaften der puren Kohlesubstrate.

\begin{tabular}{cccc}
\hline Varianten & Hersteller & Trockenrohdichte $\left[\mathrm{g} \mathrm{cm}^{-3}\right]$ & Trockenmasse [\%] \\
\hline HKpur & Pyreg & 0,384 & 56,44 \\
SKpur & Sonnenerde & 0,206 & 65,21 \\
\hline
\end{tabular}

Das Kohlensubstrat der Variante HK+N wurde jeweils zur Hälfte von beiden Herstellern bezogen. Die chemischen Eigenschaften der beiden Substrate unterscheiden sich deutlich (siehe Tab. 2.7). Die Variante des Herstellers Pyreg weist höhere Kohlenstoff- und Stickstoffgehalte und ein engeres $\mathrm{C} / \mathrm{N}$-Verhältnis auf. Daher wurden diese Kohlevarianten im Extraktions- und Topfversuch nach Hersteller getrennt geführt. Im Feldversuch wurde in die Parzellen 1/2, 2/2 und 4/2 der Variante $\mathrm{HK}+\mathrm{N}$ Kohle des Herstellers Pyreg eingearbeitet.

Tabelle 2.7: Chemische Eigenschaften der Kohlesubstrate

\begin{tabular}{cccccccccc}
\hline \multirow{2}{*}{ Varianten } & $\begin{array}{c}\mathrm{C} \\
\mathrm{g} / \mathrm{kg}\end{array}$ & $\begin{array}{c}\mathrm{N} \\
\mathrm{g} / \mathrm{kg}\end{array}$ & $\mathrm{C} / \mathrm{N}$ & $\begin{array}{c}\mathrm{K} \\
\mathrm{g} / \mathrm{kg}\end{array}$ & $\begin{array}{c}\mathrm{Ca} \\
\mathrm{g} / \mathrm{kg}\end{array}$ & $\begin{array}{c}\mathrm{Mg} \\
\mathrm{g} / \mathrm{kg}\end{array}$ & $\begin{array}{c}\mathrm{P} \\
\mathrm{g} / \mathrm{kg}\end{array}$ & $\begin{array}{c}\mathrm{S} \\
\mathrm{g} / \mathrm{kg}\end{array}$ & $\mathrm{pH}$ \\
\hline HKpur & 867,3 & 3,6 & 240 & 4,0 & 9,1 & 2,0 & 1,1 & 0,2 & 9,0 \\
SKpur & 636,2 & 12,3 & 52 & 17,2 & 6,5 & 3,7 & 6,9 & 0,6 & 9,8 \\
$\mathrm{HK}+\mathrm{N}(\mathrm{Py})$ & 823,5 & 11,5 & 72 & 4,0 & 9,3 & 1,2 & 12,0 & 9,4 & 3,8 \\
$\mathrm{HK}+\mathrm{N}(\mathrm{Se})$ & 723,6 & 7,8 & 93 & 4,4 & 13,7 & 2,1 & 31,1 & 5,6 & 2,6 \\
SK+N & 637,7 & 12,1 & 53 & 13,7 & 6,0 & 2,4 & 27,8 & 4,7 & 3,1 \\
HK+N+Ko & 353,8 & 11,1 & 32 & 11,3 & 35,9 & 6,2 & 15,3 & 4,8 & 6,6 \\
SK+N+Ko & 362,6 & 14,2 & 26 & 15,0 & 32,6 & 5,7 & 27,5 & 4,6 & 6,13 \\
\hline
\end{tabular}

Die Gehalte der Kohlen an organischen Schadstoffen und bodentoxischen Elementen wie Blei, Cadmium, Quecksilber, Kupfer, Zink, Chrom liegen unterhalb der zulässigen Grenzwerte für die Produktion von Pflanzenkohle gemäß den Richtlinien des European Biochar Consortium (EBC 2012). Die Grenzwerte entsprechen denen der Bundesbodenschutz- und Altlastenverordnung (BBodSchV 1999). Die Einhaltung der Vorgaben ist anhand von Analyseprotokollen und Produktionsberichten dokumentiert.

Der Wassergehalt der ausgebrachten Kohlesubstrate lag zwischen $25 \%$ und $43 \%$. Keimproben der Kohlesubstrate wurden an der NW-FVA durchgeführt und verliefen negativ. Mit der Kohle wurden keine keimfähigen Samen ausgebracht. Repräsentative Proben der Kohlen wurden bei der Ausbringung entnommen und gekühlt gelagert (Rückstellproben). 


\subsection{Anlage des Feldversuches}

\subsubsection{Versuchsdesign des Feldversuches}

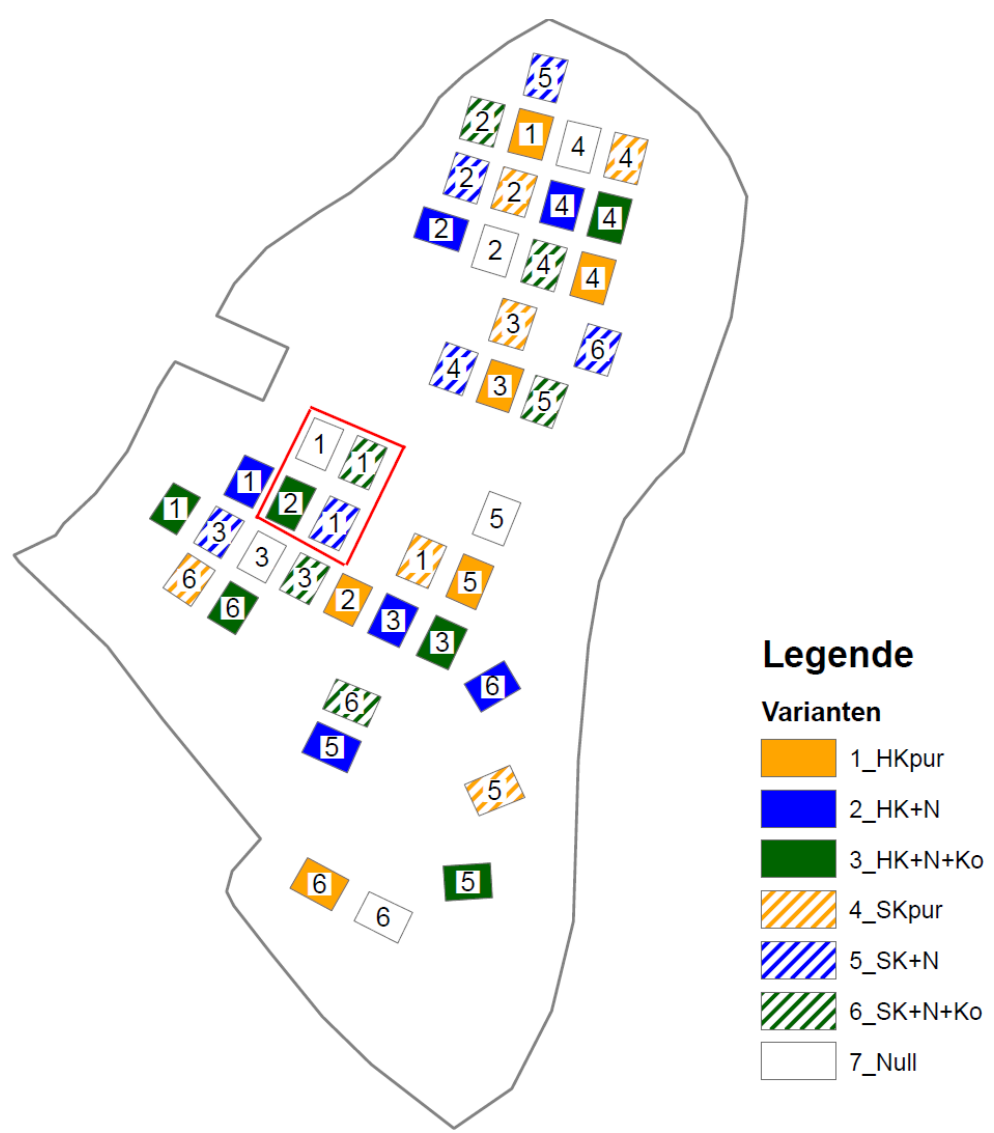

Abbildung 2.7: Parzellenplan. Rote Linien markieren den Senkenbereich mit niedrigeren pHWerten. Siehe Abschnitt „kalkhaltige Sande“ auf Seite 27

Der Versuch wurde in einem randomisierten Blockdesign in sechsfacher Wiederholung angelegt. Abbildung 2.7 gibt eine Übersicht über die Verteilung der Parzellen auf der Versuchsfläche.

\subsubsection{Vorbereitung und Bepflanzung des Versuchsfeldes}

Die Fläche wurde bereits im Herbst 2013 bis in eine Bodentiefe von 20 - 25 cm gemulcht, dabei wurde Strauch- und unterständige Baumvegetation vorwiegend aus Traubenkirsche in den Oberboden eingefräst. Der verbleibende Bestand von Altkiefern und ehemals unterständigen Stieleichen weist einen Bestockungsgrad von etwa 0,1 auf (siehe Abbildung 2.8). 


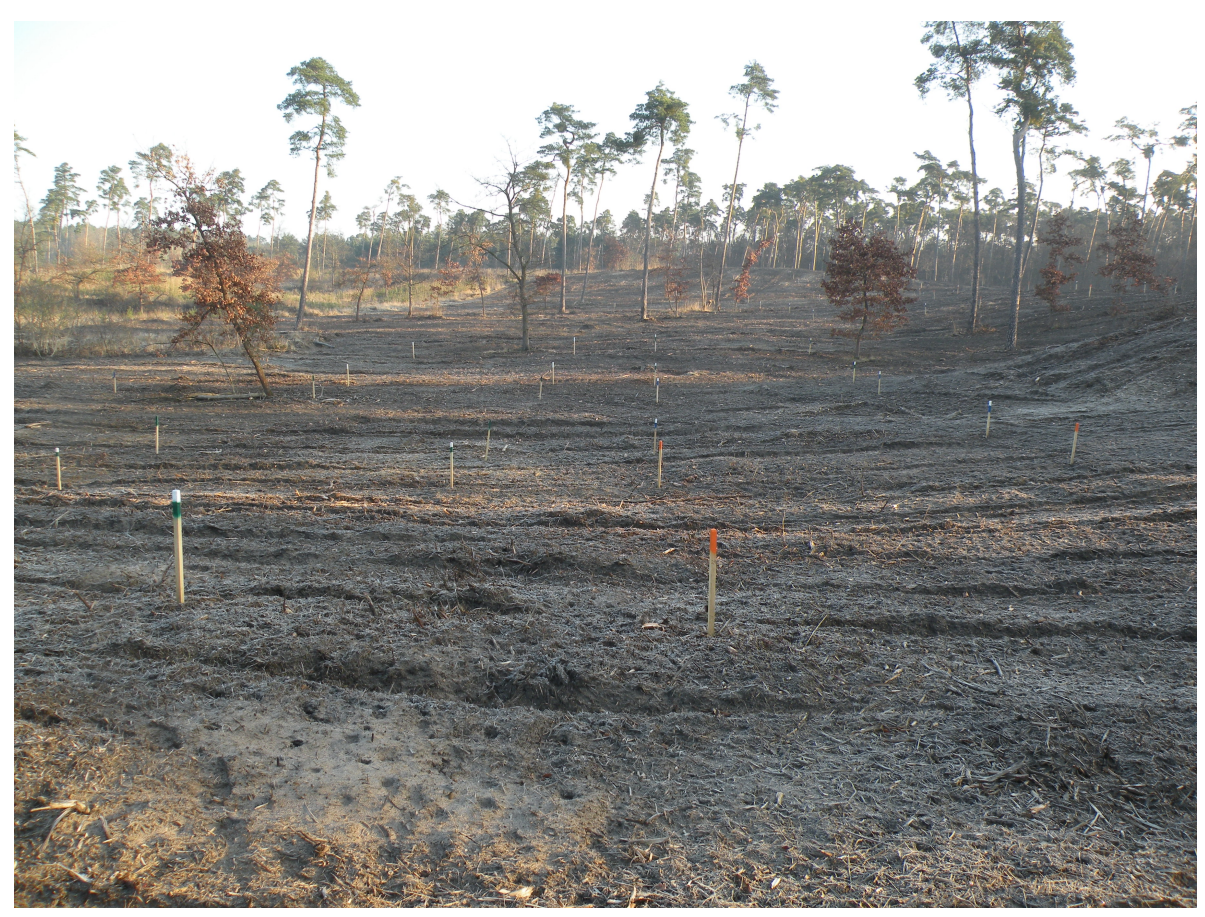

Abbildung 2.8: Flächenzustand vor Kohleneinarbeitung

Der Versuch wurde von Mitte März 2014 bis Mitte April 2014 angelegt. Dabei wurden die Parzellen per GPS eingemessen, mit Eichenpfählen an allen Ecken verpflockt und Baumhöhen und Brusthöhendurchmesser vom Altbestand ermittelt. Die Parzellen jeder Versuchsvariante sind so verteilt, dass jeweils zwei Wiederholungen auf den Kuppen der Dünen, den Dünenhängen und in den Senkenbereichen liegen. Auf stärker geneigten Hangbereichen wurden keine Versuchsparzellen eingerichtet. Zum östlich angrenzenden Bestand und zu verbliebenen Überhältern wurde ein angemessener Abstand zur Minimierung von Randeffekten durch Beschattung oder Wasserentzug gewählt.

Die Ausbringung des Kohlematerials erfolgte mit einem Düngestreuer. Nach gleichmäßiger Verteilung wurde das Material anschließend bis in $20-25 \mathrm{~cm}$ Bodentiefe eingefräst. Die Kohle wurde in einer Menge von 80 Tonnen Trockenmasse je Hektar ausgebracht, dies entspricht etwa 2,8 Masse-\%. Auch die Nullfächen wurden mit der Bodenfräse bearbeitet. Um den aufgelockerten Oberboden zu festigen, wurden alle Parzellen vor der Pflanzung abgewalzt.

Auf jeder Parzelle wurden Mitte April 40 Kiefern und 40 Hainbuchen in jeweils zwei Reihen gepflanzt (siehe Abbildung 2.9). Abweichend vom ortsüblichen maschinengestützten Pflanzverfahren wurden zweijährige Pflanzen per Hand mit dem Hohlspaten gepflanzt. Der Abstand zwischen den Pflanzen betrug $50 \mathrm{~cm}$, der Reihenabstand $2 \mathrm{~m}$. Die Hainbuchen waren unterschnitten und verpflanzt (Sortiment 1+1), die Kiefern nicht (Sortiment 2+0). Die Versuchsfläche wurde abschließend eingezäunt um den Einfluss des Wildes zu minimieren.

Aufgrund sehr hoher Ausfälle bei der Kiefer im Jahr 2014, mussten diese im Frühjahr 2015 neu gepflanzt werden. Im Frühjahr 2016 wurden abermals 40 Kiefern gepflanzt, jedoch zwischen die bisherigen Pflanzreihen, so dass letztlich Pflanzen aus drei Pflanzjahren auf den Parzellen stocken. 


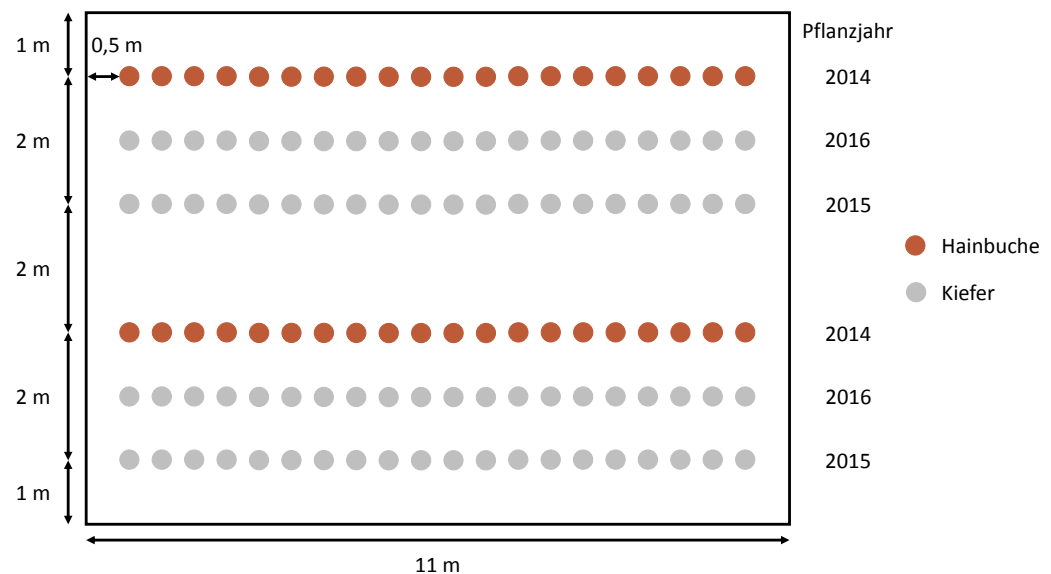

Abbildung 2.9: Pflanzplan

\subsubsection{Zusammenlegung von Versuchsvarianten}

Da bei den Hainbuchen und Kiefern auf den kohlebehandelten Parzellen in den Jahren 2014 und 2015 hohe Ausfallraten beobachtet wurden und infolgedessen die Möglichkeiten der zukünftigen Stichprobennahme begrenzt werden, ist durch die Projektbeteiligten die Zusammenlegung von Behandlungsvarianten beschlossen worden. Nach Auswertung der bis Anfang 2016 vorhandenen Daten, wurde nach Überprüfung mittels zweifaktorieller ANOVA mit den Faktoren Ausgangssubstrat (HK bzw. SK) und Behandlung (+N bzw. $+\mathrm{N}+\mathrm{Ko})$, eine Zusammenlegung der nachbehandelten Varianten nach Ausgangssubstrat beschlossen, da damit nur ein geringer Informationsverlust verbunden ist (siehe Tab. 2.8). Diese Variantenzusammenlegung gilt ausschließlich für die Wurzeluntersuchungen der Jahre 2016 und 2017. Aufgrund der Zusammenfassung ist der Stichprobenumfang dieser Varianten, bei im Jahr 2015 aufgenommenen Parametern, doppelt so groß als bei den übrigen Varianten.

Tabelle 2.8: Zusammmengelegte Versuchsvarianten

\begin{tabular}{ccc}
\hline Varianten & \multicolumn{2}{c}{ Ausgangssubstrate } \\
& Holz & Getreidespelzen \\
\hline Kohle pur (unbehandelt) & HKpur & SKpur \\
stickstoffnachbehandelt & HK/SK $+\mathrm{N}$ \\
stickstoff und kompostnachbehandelt & $\mathrm{HK} / \mathrm{SK}+\mathrm{N}+\mathrm{Ko}$ \\
Kontrolle & $\mathrm{Null}$ \\
\hline
\end{tabular}




\subsection{Messmethoden und -verfahren}

\subsubsection{Pflanze}

\subsubsection{Vollinventur mit Vitalitätsansprache}

Jeweils Ende August wurde in den Jahren 2014 bis 2017 eine Vollaufnahme aller Pflanzen durchgeführt. Dabei wurden baumspezifische Parameter wie die Baumhöhe, der Wurzelhalsdurchmesser und die Jahrestrieblänge erfasst. Der Pflanzenstatus „lebend“, „tot“ oder „nicht aufgefunden“ wurde ebenfalls notiert. Bei fehlender Belaubung bzw. Benadelung und vertrockneter Knospen wurde der Status „tot" vergeben.

Darüber hinaus wurden bei den Hainbuchen in den Jahren 2015 - 2017 etwaige Schäden vermerkt und die Vitalität der Bäume hinsichtlich ihres Belaubungszustandes angesprochen. Die Kategorie „starker Blattverlust“ wurde bei einem Blattverlust von mehr als $30 \%$ vergeben (siehe Abb. 2.10 (links)), tatsächlich lag der Blattverlust oftmals bei über $90 \%$. Bäume mit geringeren Blattverlusten von maximal 30 \% wurden in die Kategorie „mittlerer Blattverlust“ eingeordnet. In der Kategorie „,geringer Blattverlust" sind lediglich Pflanzen mit eingerollten Blättern oder vertrockneten Blatträndern zu finden. Pflanzen ohne sichtbare Schäden sind in die Kategorie „gesund“ eingestuft worden.

Bei der Kiefer wurde die Vitalität anhand des Benadelungszustandes in den Jahren 2015 und 2016 beurteilt. Die Kategorie „starke Nadelverbraunung“ wurde bei einer Nadelverbraunung von mehr als $30 \%$ vergeben (siehe Abb. 2.10 (rechts)). Kiefern mit geringerer Nadelverbraunung sind in der Kategorie „mittlere Nadelverbraunung/-vergilbung“ eingeordnet. In die Kategorie „geringe Nadelvergilbung“" wurden Pflanzen mit geringen Anteilen von Nadelvergilbungen eingestuft.
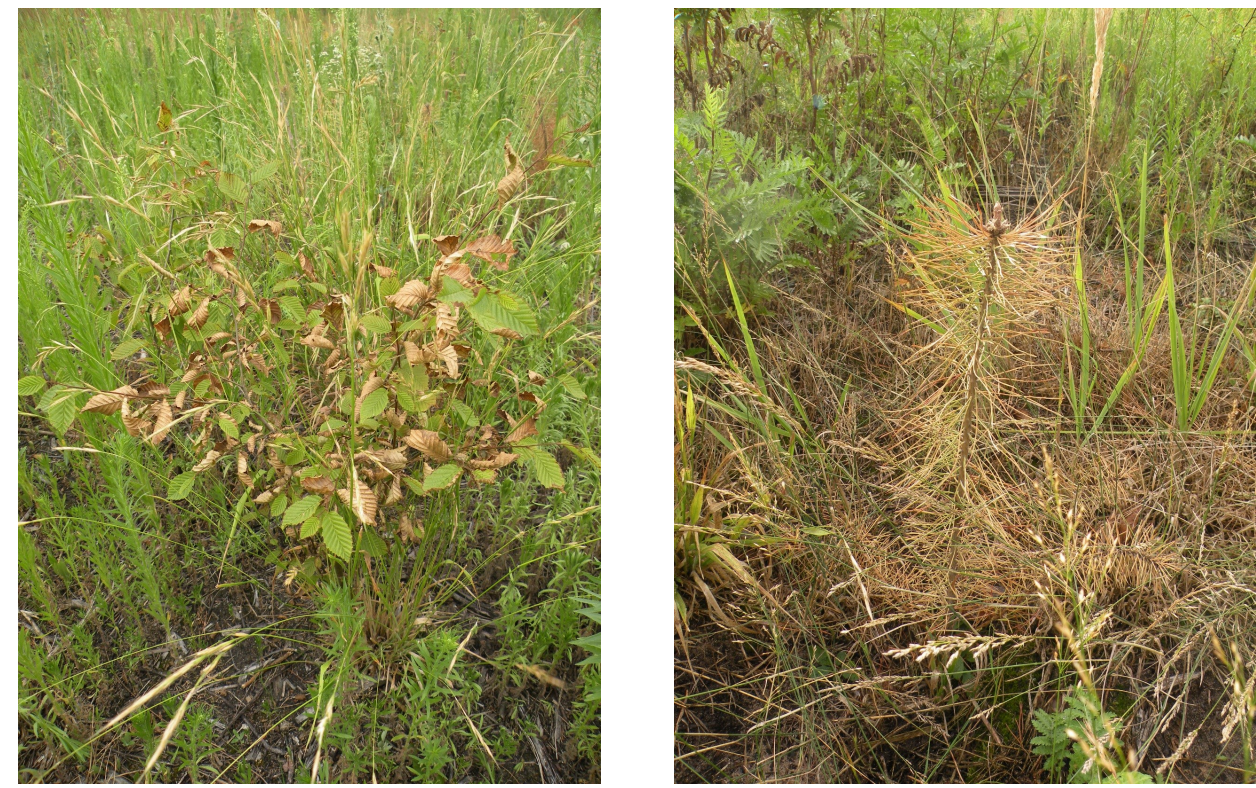

Abbildung 2.10: Beispiel der Vitalitätskategorie „starker Blattverlust“ an Hainbuche (links) bzw. „starke Nadelverbraunung“" an Kiefer (rechts) 


\subsubsection{Begleitvegetation}

Pflanzen sind den Umweltfaktoren als ortsfeste Organismen stetig ausgesetzt. Sie eignen sich daher als vielseitige Bioindikatoren. Zeigerpflanzen reagieren auf Veränderungen der Standortsfaktoren mit stärkerem oder schwächerem Wuchs bzw. Vermehrung oder mit dem Verschwinden. Der Zustand der Begleitvegetation wurde auf den einzelnen Parzellen nach Art und anteiligem Deckungsgrad aufgenommen. Der Deckungsgrad wurde in $5 \%$-Stufen geschätzt. Alle Pflanzenarten mit einem Deckungsgrad von unter $5 \%$ wurden nachrichtlich aufgeführt und ein pauschaler Deckungsgrad von 0,2 \% zugeordnet. Zusätzlich wurde für alle Arten mit mehr als $5 \%$ Deckungsgrad je Parzelle die mittlere Sprosslänge ermittelt.

Basierend auf den Daten der Vegetationsaufnahmen wurden die durchschnittlichen Stickstoffvorräte der Vegetation (Hainbuchen, Kiefern und Begleitvegetation) auf den Versuchsparzellen mit dem PhytoCalc-Modell berechnet. Das Modell wurde sowohl für den Einsatz in geschlossenen Wäldern als auch unter Freiflächenbedingungen parametrisiert und arbeitet mit Biomasse- und Elementschätzfunktionen für Arten und Artengruppen (Bolte 1999; Bolte et al. 2002; Bolte et al. 2009; Klinck und Fröhlich 2009; Heinrichs et al. 2010).

\subsubsection{Probenahme von Pflanzen, morphologische Messungen und Probenvorbereitung für die chemische Analyse}

Jeweils im Oktober der Jahre 2015 bis 2017 wurden zufällig ausgewählte Pflanzen beider Baumarten aus jeweils drei Parzellen je Versuchsvariante ausgegraben. Im Jahr 2015 wurden die ursprünglich sieben Versuchsvarianten einzeln beprobt, während im Jahr 2016 den Parzellen der zusammengelegten Versuchsvarianten (siehe Abschnitt „Zusammenlegung von Versuchsvarianten" auf Seite 32) Pflanzen entnommen wurden. Die Parzellen wurden per Zufall ausgewählt, mussten jedoch noch genügend Pflanzen für die Probenahme aufweisen. Pro Baumart wurden jeweils drei lebende Pflanzen ausgewählt und mit Wurzelsystem vorsichtig ausgegraben, um Wurzelverletzungen und Feinwurzelabrisse zu vermeiden.

Im Feld erfolgte die Trennung in die Kompartimente „Wurzel“, „Spross“ bzw. bei der Kiefer in „diesjähriger Trieb“ und „letztjähriger Trieb“ erfolgte. Die Trennung und die Längenbestimmung der Wurzelkompartimente Feinwurzel (unter $2 \mathrm{~mm}$ ), Mittelwurzel $(2-5 \mathrm{~mm}$ ) und Grobwurzel (über $5 \mathrm{~mm}$ ) wurde im Labor durchgeführt. Ebenso die Trennung der Nadeln von den dies- und letztjährigen Kieferntrieben.

Die Elementgehalte der Pflanzenkompartimente geben einen Einblick in den Ernährungsstatus der untersuchten Pflanzen. Für die Bestimmung der Blattelementgehalte wurden voll ausgereifte Hainbuchenblätter ohne sichtbare Schäden verwendet. Die Lagerung, Vorbereitung und Analyse der Pflanzen- als auch der Bodenproben erfolgte nach Standardmethoden der forstlichen Analytik (König 2009). Nach Trocknung der Proben im Labor bei $60^{\circ} \mathrm{C}$ wurden die Trockenmassen aller Pflanzenkompartimente ermittelt und die Blatt- und Nadelproben sowie die Feinwurzelproben chemisch analysiert.

Für die Bewertung der Blatt-/Nadelelementgehalte wurde die Einteilung nach Krauß und Heinsdorf (2005) genutzt, da hier auch Werte für Hainbuche vorliegen. Für die Bewertung der harmonischen Elementverhältnisse sind die Einstufungen nach Mellert und Göttlein (2012) (Buche und Kiefer) und Flückiger und Braun (2003) (Buche) angewendet worden. Die Ergebnisse der Hainbuche wurden mit den harmonischen Verhältniswerten der Buche verglichen und bei Unterschieden zwischen den Referenzsystemen der strengere Wert zur Beurteilung herangezogen. 
Untersuchungen zur Mykorrhizierung der Feinwurzeln Als Mykorrhiza werden symbiontische Zusammenschlüsse von Pflanzenwurzeln und spezialisierten Pilzen zum beiderseitigen Nutzen bezeichnet. An Bäumen kommen vor allem Ektomykorrhizen vor, die mit ihren Hyphen in den Interzellularäumen der Wurzeln das Hartig'sche Netz ausbilden. Die Feinwurzeloberfläche wird von einem Myzelmantel umschlossen, der den Pflanzen einen Zugang zu einem größeren Bodenvolumen verschafft. Die Nährstoffversorgung und die Trockenresistenz von mykorrhizierten Pflanzen kann somit erhöht werden (Pena und Polle 2014). Besonders in nährstoffarmen Böden kommt den Mykorrhizapilzen eine große Rolle in der Nährstoffaufnahme der Pflanzen zu (Lyr et al. 1967; Mikola 2012).

Der Transport der Feinwurzeln erfolgte 2016 für die anschließenden Mykorrhizauntersuchungen in feuchten Papiertüchern und Leinenbeuteln. Die Feinwurzeln beider Baumarten wurden im Oktober 2016 durch einen Masterstudenten auf Ektomykorrhizierung untersucht. Zur Bestimmung des Mykorrhizierungsgrades wurden an jeder Pflanze mindestens 100 Wurzelspitzen (WS) unter einem Binokular morphologisch ausgezählt. Dabei wurden die Wurzelspitzen in die Klassen „trocken“, „,vital“ (aber nicht mykorrhiziert) und „mykorrhiziert“ eingeteilt. Zur Unterscheidung dienten Merkmale wie Oberflächenbeschaffenheit, Breite und Farbe der Wurzelspitzen. So sind dunkle Wurzelspitzen mit unregelmäßiger runzelig/knittriger Oberfläche Merkmal trockener Wurzelspitzen. Als mykorrhiziert galten Wurzelspitzen mit glatter Oberfläche, welche an der Spitze breiter waren als an der Basis. Nicht-mykorrhizierte Wurzelspitzen sind durch die gleiche Breite von Basis und Spitze sowie den meist sichtbaren Wurzelhaaren zu erkennen.

Der Mykorrhizierungsgrad ergibt sich nach folgender Formel:

$$
\text { Mykorrhizierungsgrad }=\frac{\text { Anzahl mykorrhizierter Wurzelspitzen }(W S)}{\text { Anzahl vitaler } W S+\text { Anzahl mykorrhizierter } W S} * 100
$$

Des Weiteren wurden einige Ektomykorrhiza-Arten durch Mitarbeiter in der Abteilung Forstbotanik und Baumphysiologie am Büsgen-Institut der Georg-August-Universität Göttingen morphologisch bestimmt.

\subsubsection{Chlorophyllgehalte (SPAD)}

Der relative Chlorophyllgehalt der Hainbuchenblätter wurde mit einem SPAD 502plus ChlorophyllMessgerät mittels Fluoreszenzmethode bestimmt (Konica Minolta Optics, Tokyo, Japan). Dabei wird der Anteil des vom Blatt transmittierten (oder absorbierten) Lichts bei den Wellenlängen $650 \mathrm{~nm}$ (rot) und $940 \mathrm{~nm}$ (Infarot) gemessen. Als Resultat wird ein Wert zwischen 1 und 100 (SPAD-Wert) ausgegeben, der Informationen über den relativen Blatt-Chlorophyllgehalt enthält (Chang und Robison 2003).

In den Vegetationszeiten 2014 und 2015 wurden mit monatlichem Abstand 5 Pflanzen pro Parzelle gemessen, wobei 10 Einzelmessungen pro Pflanze an Blättern im oberen Kronenbereich durchgeführt wurden. Im Topfversuch wurden alle Hainbuchen mittels 10 Einzelmessungen pro Pflanze gemessen.

\subsubsection{Blattflächen und Blattmassen}

Im Rahmen einer Blattbonitur wurden 2015 und 2016 die Blätter gescannt und mit der Software WinFoliar (Regent Instruments Inc. Quebec, Canada) die durchschnittliche Einzelblattfläche analysiert. 


\subsubsection{Blattwassergehalt und -trockenmasse}

Ein geregelter Pflanzenstoffwechsel kann nur bei ausreichender Versorgung mit Wasser und Nährstoffen ablaufen. Wasser dient dabei als Lösungs- und Transportmittel, zur Aufrechterhaltung des Turgors und ist an vielen chemischen Umsetzungen beteiligt. Der Blattwassergehalt ist ein Indikator des aktuellen Wasserstatus einer Pflanze (Sinclair und Ludlow 1985). Da der Wasserbedarf ausgelöst durch die Transpiration im Tagesverlauf die Wassernachleitung aus den Wurzeln übersteigt, entspricht die Wassersättigung der Blätter nicht mehr dem maximal möglichen Wert. Der relative Wassergehalt kann als ein Indikator des aktuellen Wasserstatus einer Pflanze interpretiert werden (Sinclair und Ludlow 1985).

Im August bzw. September 2015 und Juli bzw. August 2016 wurden pro Parzelle drei Hainbuchen beprobt und in einer Mischprobe zusammengefasst. Die frischen Blätter wurden noch im Feld gewogen (Frischgewicht), danach in PE-Plastikdosen transportiert und bei $60^{\circ} \mathrm{C}$ bis zur Gewichtskonstanz getrocknet und erneut gewogen (Trockengewicht). Der Wassergehalt der Blätter bezogen auf das Frischgewicht berechnet sich wie folgt:

$$
\text { Wassergehalt }[\%]=\frac{\text { Frischgewicht }- \text { Trockengewicht }}{\text { Frischgewicht }} * 100
$$

\subsubsection{Stomatäre Leitfähigkeit}

Die stomatäre Leitfähigkeit wurde über ein steady-state Porometer AP4 (Delta-T Devices, Cambridge, England) ermittelt. Dazu wird ein Blatt in eine kleine Kammer (Küvette) eingespannt und die relative Luftfeuchtigkeit erfasst. Die Änderung der Luftfeuchte wird als Maß für die Transpiration der Pflanze angesehen (Steubing und Fangmeier 1992).

Die stomatäre Leitfähigkeit wurde in den Monaten Juli und August der Jahre 2015 und 2016 in 3-facher Wiederholung pro Versuchsvariante an jeweils 2 Pflanzen pro Parzelle bestimmt. 


\subsubsection{Boden}

\subsubsection{Bodentemperatur und Bodenfeuchte}

Die Bodentemperatur kann von der Lufttemperatur deutlich abweichen. Dies ist bei ähnlichen Böden abhängig von Sonneneinstrahlung und Albedo. Albedo bezeichnet dabei den reflektierten Anteil der Einstrahlung, der nicht vom Boden oder Pflanzen zurückgehalten wird. Der vom Boden absorbierte Teil der Strahlung wird in Wärme umgewandelt die den Boden und die darüberliegende Luft erwärmt. Vegetation vermindert die tageszeitlichen Temperaturschwankungen, da sie einen Teil der Sonneneinstrahlung abfängt (Wild 1995).

Die Messung der Bodenfeuchte erfolgte über Sonden, die nach dem Time-Domain-Reflectometry (TDR)-Verfahren die Bodenfeuchte ermitteln (Topp et al. 1980). Dabei wirken zwei Elektroden als Kondensator und der sie umgebende Boden als Dielektrikum. Über einen Oszillator wird eine Schwingung von $80 \mathrm{MHz}$ an den Kondensator angelegt und ein Ausgangssignal erzeugt, welches proportional zur Dielektrizitätskonstante des Bodens ist. Die Dielektrizitätskonstante von Wasser ist größer als die von Luft, Mineralboden oder organischer Substanz. Damit können Veränderungen im Wassergehalt des Bodens durch den Sensor gemessen und mit der Bodenfeuchte korreliert werden.

Im Zeitraum von Mitte 2015 bis Mitte 2017 wurde die Bodenfeuchte auf jeweils einer Parzelle jeder Variante in 30 und $90 \mathrm{~cm}$ Bodentiefe kontinuierlich aufgezeichnet. Die Bodentemperatur wurde auf den gleichen Parzellen in $30 \mathrm{~cm}$ Bodentiefe gemessen. Längere Ausfallzeiträume des Messsystems, in denen keine Daten aufgezeichnet wurden, gab es von Dezember 2015 bis Mai 2016, Mitte August bis Mitte September 2016 und im Januar 2017. Die Bodenfeuchte- und Temperatursensoren (Spectrum Technologies, Illinois, USA) wurden vor dem Einbau im Feld auf Messabweichungen verglichen, dabei wurde keine Notwendigkeit der Kalibrierung erkannt.

\subsubsection{Gravimetrische Wassergehaltsbestimmung des Feldbodens}

Zur Validierung der per TDR-Sensoren gemessenen Bodenfeuchte wurde an sieben Terminen von Mitte 2016 bis März 2017 der Wassergehalt gravimetrisch ermittelt. Dazu wurden Bodenproben aus $10-30 \mathrm{~cm}$ bzw. 80 - $90 \mathrm{~cm}$ Bodentiefe gezogen, im Feld gewogen (Frischgewicht), bei 105 ${ }^{\circ} \mathrm{C}$ bis zur Gewichtskonstanz getrocknet und nach Abkühlung im Exsikkator erneut gewogen (Trockengewicht). Der gravimetrische Wassergehalt des Bodens errechnet sich durch folgende Formel:

$$
\text { Wassergehalt }[\%]=\frac{\text { Frischgewicht }- \text { Trockengewicht }}{\text { Frischgewicht }} * 100
$$

Die Umrechnung in den volumetrischen Wassergehalt erfolgt durch Multiplikation des gravimetrischen Wassergehaltes mit der Trockenraumdichte.

\subsubsection{Wasser-Versickerungszeit}

Die Geschwindigkeit, mit der Niederschläge im Boden versickern, ist stark von Bodenart, Verdichtung und der Bodenversiegelung abhängig. Darüberhinaus ist der Anteil quellförmiger Substanzen, wie Ton- und Humuskolloide von großer Bedeutung. Je höher die aktuelle Bodenfeuchte, umso geringer die jeweilige Versickerungsrate des Bodens (Steubing und Fangmeier 1992).

Die Messungen wurden an drei Terminen im Sommer 2015 durchgeführt. In jeder Behandlungsvariante wurden drei Parzellen zufallsausgewählt. An vegetationsfreien Stellen ist ein Stechzylinder mit einem Volumen von $250 \mathrm{ml}$ etwa $2-3 \mathrm{~cm}$ tief vorsichtig eingedrückt bzw. eingeschlagen worden. Der Stechzylinder wurde mit $107 \mathrm{ml}$ Wasser gefüllt und die Zeit bis zur vollständigen 
Versickerung des zugegebenen Wassers mit einer Stoppuhr gemessen. Wenn nach 5 Minuten noch Wasser über der Bodenoberflächestand, wurde die Messung abgebrochen und als Maximalwert 300 Sekunden Versickerungszeit für $107 \mathrm{ml}$ Wasser notiert.

\subsubsection{Gewinnung der Bodenlösung}

Die Bodenlösung nimmt innerhalb des Stoffkreislaufes als Umschlagplatz eine zentrale Stellung ein. Durch sie sind die Prozesse Mineralisierung und Nährstoffaufnahme miteinander verbunden (Rapp 1991). Die Endprodukte der Zersetzung organischer Substanz werden von der Bodenlösung aufgenommen. Aus der Bodenfestphase können durch Verwitterung, Auflösung und Austauschreaktionen gelöste Elemente in die Bodenlösung übergehen. Der Austrag findet durch Pflanzenaufnahme oder Auswaschung statt. Daher ist die Zusammensetzung der Bodenlösung stark von der Jahreszeit abhängig und längerfristige Beobachtungen über mehrere Jahre notwendig, um robuste Aussagen treffen zu können (Standortskartierung 2003). Die Zusammensetzung der Bodenlösung ist ein wichtiger Indikator für die Nährstoffversorgung und für das Auftreten von toxischem Stress (Meesenburg et al. 2016).

Für die Gewinnung der Bodenlösung in $90 \mathrm{~cm}$ Bodentiefe werden jeweils 21 Parzellen (drei Wiederholungen je Versuchsvariante) mit je einer Saugkerze beprobt. Zusätzlich sind zur Abbildung der räumlichen Variabilität auf Parzelle 6/5 vier Saugkerzen installiert worden.

Da die Vorlauf- bzw. Konditionierungszeit für die installierten Saugkerzen zu gering war, um einen ungestörten Bodenzustand in der Umgebung der Saugkerzen zu erreichen, wurde im Winter und Frühjahr 2015 in monatlichem Abstand das Probenahmeverfahren nach Wilpert et al. (2011) angewandt. Dabei werden die Saugkerzen eingebaut bzw. umgesetzt, unmittelbar ein Unterdruck angelegt und die innerhalb von 3-4 Tagen gewonnene Bodenlösung analysiert. Die durch Sauerstoff gesteuerte Mineralisierung läuft erst nach 4-5 Tagen an. Daher ist innerhalb eines Zeitraumes von 3-4 Tagen ein ungestörter Bodenzustand mit fragmentfreier Bodenlösung anzunehmen. Durch den kurzen Probenahmezeitraum von 3-4 Tagen in Verbindung mit dem sandigen Boden ist eine starke Abhängigkeit von Regenereignissen gegeben, um genügend Bodenlösung zu gewinnen (Unold 2000). Ab Mai 2015 war der Bodenwassergehalt bereits zu gering, um mit dieser Methode genügend Bodenlösung zu erhalten. Ab dem Jahr 2016 wurde an die Saugkerzen kontinuierlich Unterdruck angelegt und die gewonnene Bodenlösung alle 14 Tage abgefüllt und zu einer monatlichen Mischprobe vereinigt. Bis zur Analyse wurde die Bodenlösung gekühlt aufbewahrt. 


\subsection{Topfversuch}

Während langfristige Effekte besser im Feldversuch untersucht werden können, bietet ein Topfversuch unter kontrollierten Bedingungen die Möglichkeit, initiale direkte Effekte auf Boden und Pflanzen zu beobachten (Novak et al. 2009).

Gerade in der Anfangsphase des Feldversuches im Jahr 2014 hat das Zusammenwirken mehrerer Effekte zu einem schnellen Absterben einiger Versuchspflanzen (v.a. Kiefer) geführt. Um eine Trennung und Quantifizierung der mortalitätsbestimmenden Faktoren zu ermöglichen, ist es sinnvoll, die Anfangsphase unter kontrollierten Bedingungen zu simulieren. Daher wurde im Jahr 2017 ein Topfversuch im Gewächshaus der NW-FVA in Hann. Münden eingerichtet und durchgeführt.

\subsubsection{Versuchsanlage}

Das Ausgangssubstrat für den Topfversuch wurde von der Versuchsfläche in Südhessen gewonnen. Jeweils 3,5 kg Ausgangssubstrat (Trockenmasse) und 96 g TM Kohle aus Rückstellproben des Feldversuches wurden vermischt und in einen 3 Liter Pflanztopf gefüllt. Die Menge der zugesetzten Kohle entsprach der eingebrachten Kohlemenge im Feldversuch $\left(80 \mathrm{t} \mathrm{ha} \mathrm{h}^{-1}\right)$. Aufgrund der herstellerbedingt unterschiedlichen chemischen Eigenschaften der Kohlevariante HK+N (siehe Abschnitt 2.2.3 auf Seite 29) wurde diese Variante im Topf- und im Extraktionsversuch in zwei Versuchsvarianten getrennt beobachtet $(\mathrm{HK}+\mathrm{N}(\mathrm{Py})$ bzw. HK+N (Se). Für jede Versuchsvariante und Baumart stehen vier Wiederholungen zur Verfügung. Die 64 Töpfe wurden mit Hainbuche oder Kiefer bepflanzt. In einer Aufsättigungsphase wurden die bepflanzten Töpfe in wassergefüllte Untersetzer gestellt, um eine Aufsättigung mit Wasser ohne etwaige Wasser- und Stoffverluste des Substrates zu ermöglichen. Nach der Aufsättigung wurden die Pflanztöpfe randomisiert im Gewächshaus aufgestellt. Als Verdunstungsschutz dienten Pflanzscheiben mit denen die Pflanztöpfe abgedeckt wurden (siehe Abb. 2.11).

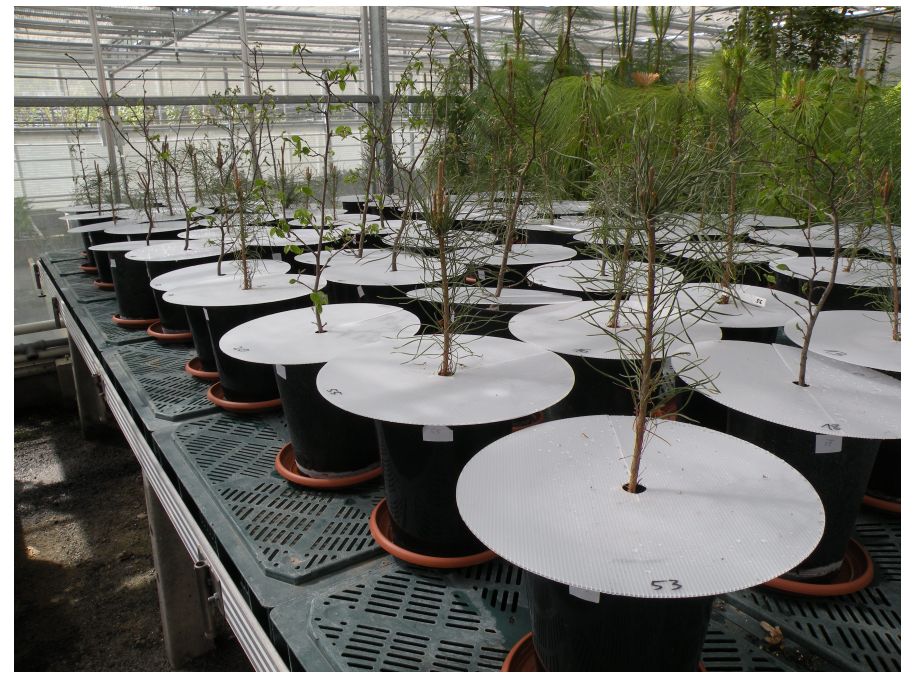

Abbildung 2.11: Topfversuch im Gewächshaus. Randomisiert aufgestellte Pflanztöpfe mit Verdunstungsschutz und Sickerwasserauffangschalen. 


\subsubsection{Durchführung}

Zur Ermittlung der Stoffausträge wurde das Sickerwasser aufgefangen und analysiert. In wöchentlichem Abstand wurden die Pflanzen mit einer definierten Menge an Leitungswasser gegossen, bis sich etwa $100 \mathrm{ml}$ Sickerwasser in den Untersetzern gesammelt hat. Jeweils zwei zeitlich aufeinanderfolgende Proben wurden zu einer Mischprobe vereinigt, die somit einen Zeitraum von 14 Tagen repräsentiert. Die Proben wurden bis zur ionenchromatographischen Analyse auf Nitrat und Ammonium gekühlt gelagert.

Nach der 8-wöchigen Bewässerungsphase bis zum Abschluss des Knospenaustriebs, schloss sich die Trockenphase an. In dieser Phase wurden die Töpfe wöchentlich gewogen, um den Transpirationsverlust zu quantifizieren. Die Pflanzen wurden mit folgendem Boniturschema (siehe Tab. 2.9) wöchentlich bonitiert.

Tabelle 2.9: Boniturstufen und Beschreibung von Schadsymptomen an Blättern und Nadeln.

\begin{tabular}{|c|c|c|}
\hline Stufe & Schadsymptome Hainbuche & Schadsymptome Kiefer \\
\hline 0 & ohne Symptome & \\
\hline 1 & $\begin{array}{l}<50 \% \text { der Blätter zwischen den Blattnerven ver- } \\
\text { gilbt }\end{array}$ & Nadelvergilbung 2./3. Jahrgang \\
\hline 2 & $\begin{array}{l}>50 \% \text { der Blätter zwischen den Blattnerven ver- } \\
\text { gilbt }\end{array}$ & $\begin{array}{l}\text { Nadelvergilbung } 1 . \quad \text { Jahrgang } \\
\text { (mit Nadelverbraunung, - -verlust } \\
\text { älterer Jahrgänge) }\end{array}$ \\
\hline 3 & einzelne Blätter hängen schlaff (beginnende Welke) & \\
\hline 4 & Blattnekrosen an den Rändern weniger Blätter & \\
\hline 5 & $<50 \%$ der Blätter nekrotisch, eingerollt, trocken & $\begin{array}{l}\text { Nadelverbraunung } \\
\text { zen) 1. Jahrgang }\end{array}$ \\
\hline 6 & $>50 \%$ der Blätter nekrotisch, eingerollt, trocken & \\
\hline 7 & Blattfall beginnend & \\
\hline 8 & \multicolumn{2}{|c|}{ weitestgehend kahl bzw. abgestorben } \\
\hline
\end{tabular}

Der SPAD-Gehalt der Hainbuchenblätter (siehe Abschnitt 2.4.1.4 auf Seite 35) wurde an fünf Terminen untersucht. Die Pflanzen wurden in die Kompartimente Wurzel und Spross bei Hainbuche und diesjähriger Spross und Nadeljahrgang, letztjähriger Spross und Nadeljahrgang bei Kiefer getrennt, um nach Trocknung bei $60^{\circ} \mathrm{C}$ die jeweiligen Trockenmassen zu bestimmen.

\subsection{Extraktionsversuch}

\subsubsection{Versuchsanlage}

Um die Mineralisierungsdynamik der Kohle und die Stoffausträge in der Initialphase des Versuches nachzuvollziehen, wurde ein Extraktionsversuch nach Stanford und Smith (1972) und Bauhus (1996) durchgeführt. Die Langzeitinkubation zur Schätzung der Stickstoffmineralisation kann als ein Indikator der Stickstoffversorgung gelten (Stanford und Smith 1972; Bundy und Meisinger 1994).

Aus Feldbodenmaterial und Rückstellproben der Kohle wurde ein Substrat angemischt, das dem Feldsubstrat in Mischungsverhältnis Feldboden zu Kohle gleicht. Jede Versuchsvariante wurde 2-fach wiederholt. Das Substrat wurde bei $8{ }^{\circ} \mathrm{C}$ inkubiert. Zur Bestimmung des NettoStickstoffmineralisationspotenzials wurde die mineralische Stickstoffkonzentration am letzten 
Messtermin herangezogen. Die Inkubationsdauer seit dem vorletzten Probetermin betrug 6 Wochen. Die Konzentrationen wurden in $\mathrm{mg} \mathrm{g}^{-1}$ Trockensubstanz der Einwaage umgerechnet und anschließend über die Trockenrohdichte des Oberbodens auf den Flächenwert kg ha ${ }^{-1}$ bezogen. Diese Größe stellt das Mineralisierungspotenzial bei $8{ }^{\circ} \mathrm{C}$ dar und wird dem mittleren Jahreswert im Feldversuch (in-situ) gleichgesetzt.

\subsubsection{Durchführung}

Die Extraktionen fanden zu Versuchsbeginn und nach 0, 1, 2, 4, 6, 10, 16, 20 und 25 Wochen statt. An den Extraktionsterminen wurde mit $20 \mathrm{ml} \mathrm{0,01}$ molarer $\mathrm{CaCl}_{2}$-Lösung extrahiert. Die Extrakte wurden nach Vorverdünnung (bis zu 1:50) ionenchromatografisch auf Nitrat und Ammonium untersucht. Um eine Nährstofflimitierung der Mikroorganismen zu vermeiden, wurde der Boden nach jeder Extraktion mit einer stickstofffreien Nährlösung gespült, die aus $\mathrm{Ca}\left(\mathrm{H}_{2} \mathrm{PO}_{4}\right)_{2}$, $\mathrm{MgSO}_{4}, \mathrm{KH}_{2} \mathrm{PO}_{4}$ und $\mathrm{K}_{2} \mathrm{SO}_{4}$ bestand. Zwischen den Extraktionsterminen wurden die Extraktionsbecher abgedeckt, um Feuchtigkeitsverluste zu vermeiden.

\subsection{Sickerwassermodellierung}

Die Sickerwasserraten wurden mit dem hydrologischen Modell LWF-Brook90 (Hammel und Kennel 2001) in Verbindung mit dem R-Paket „brook90r“ (Schmidt-Walter 2017) berechnet. Da die Varianten HKpur und SKpur in ihren Retentionskurven (siehe Abb. 3.2 auf Seite 46) und in anderen für die Modellierung relevanten Parametern weitgehend übereinstimmen, wurde die Variante SKpur nicht separat modelliert. Die Modellergebnisse der Variante HKpur können auf die Variante SKpur übertragen werden. Ebenso unterscheiden sich die stickstoff- $(+\mathrm{N})$ bzw. kompostnachbehandelten $(+\mathrm{N}+\mathrm{Ko})$ Varianten in ihren für die Modellierung relevanten Parametern kaum voneinander. So weist beispielsweise die Begleitvegetation kaum Unterschiede zwischen diesen Varianten auf. Daher können die modellierten Ergebnisse der Variante HK+N+Ko auf die stickstoff- $(+\mathrm{N})$ bzw. kompostnachbehandelten $(+\mathrm{N}+\mathrm{Ko})$ Varianten übertragen werden.

\subsubsection{Parametrisierung}

Es wurden die Klimadaten der nahegelegenen DWD-Klimastation in Mannheim genutzt. Der Dampfdruck der Luft als Eingangsvariable des Sickerwassermodells wurde aus der Lufttemperatur und der relativen Luftfeuchte berechnet. Zunächst wurde in einem Zwischenschritt der Sättigungsdampfdruck e $\mathrm{e}^{0}$ (maximaler Dampfdruck bei Sättigung) für die tägliche Minimal- $\left(e_{\text {Tmin }}^{0}\right)$ bzw. Maximaltemperatur $\left(e_{T \max }^{0}\right)$ mit folgender Formel berechnet.

$$
e^{0}=0.6108 * e^{\frac{17.27 * T}{T+237.3}}
$$

mit $\mathrm{T} \quad$ Lufttemperatur $\left[{ }^{\circ} \mathrm{C}\right]$

Anschließend kann aus dem arithmetischen Mittel des Sättigungsdampfdruckes der Maximalbzw. Minimaltemperatur und der Luftfeuchte der mittlere Dampfdruck berechnet werden:

$$
e_{a}=\frac{R H_{\text {mean }}}{100} * \frac{e^{0}\left(T_{\max }\right)+e^{0}\left(T_{\min }\right)}{2}
$$

$$
\begin{array}{ll}
\mathrm{e}_{\mathrm{a}} & \text { mittlerer Dampfdruck }[\mathrm{kPa}] \\
\mathrm{RH}_{\text {mean }} & \text { relative Luftfeuchtigkeit [\%] } \\
\mathrm{e}^{0} & \text { Sättigungsdampfdruck [kPa] }
\end{array}
$$


Aus den ermittelten Retentionskurven wurden die bodenphysikalischen Kennwerte nach Wessolek et al. (2009) abgeleitet. Das Modell wurde mittels Vegetationsdaten aus der jährlichen Inventur parametrisiert (Deckungsgrad der Begleitvegetation, Pflanzenhöhen, Wurzelmorphologie). Die Parametrisierung der Bestände erfolgte ausgehend von den Standard (default)-Einstellungen und führte zu den in Tabelle A.4 (im Anhang) aufgeführten Parametereinstellungen in den Einzelfunktionen. Nicht aufgeführte Parameter entsprechen den Standardeinstellungen.

\subsubsection{Validierung}

Das Modellergebnis der Nullvariante wurde anhand der Wassergehalte in $20 \mathrm{~cm}$ und $100 \mathrm{~cm}$ Bodentiefe einer südhessischen Level II-Fläche (Kiefer, Hessisches Ried) validiert (siehe Abb. 2.12 und 2.13). Das Modell bildet die Wassergehalte in $100 \mathrm{~cm}$ Bodentiefe während der Vegetationszeit gut ab. In der Nichtvegetationszeit bestehen leichte Abweichungen bis max. 7 \%, wobei im Jahr 2014 sehr große Unterschätzungen der Bodenwassergehalte zu beobachten sind. Die Wassergehalte in $20 \mathrm{~cm}$ Bodentiefe werden in der Nichtvegetationszeit durch das Modell gut abgebildet. In der Vegetationszeit unterschätzt das Modell die Bodenwassergehalte auf der Level II-Fläche.

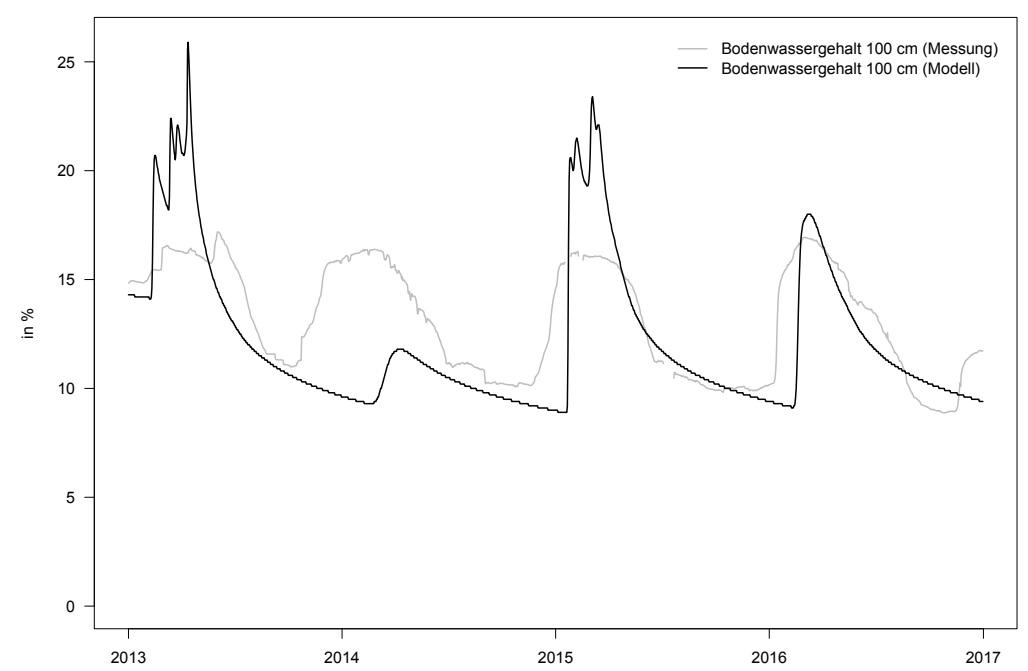

Abbildung 2.12: Vergleich der gemessenen (graue Linie) mit den modellierten (schwarze Linie) Bodenwassergehalten in $100 \mathrm{~cm}$ Bodentiefe der Jahre 2013 - 2016 auf der Level II-Fläche Hessisches Ried. 


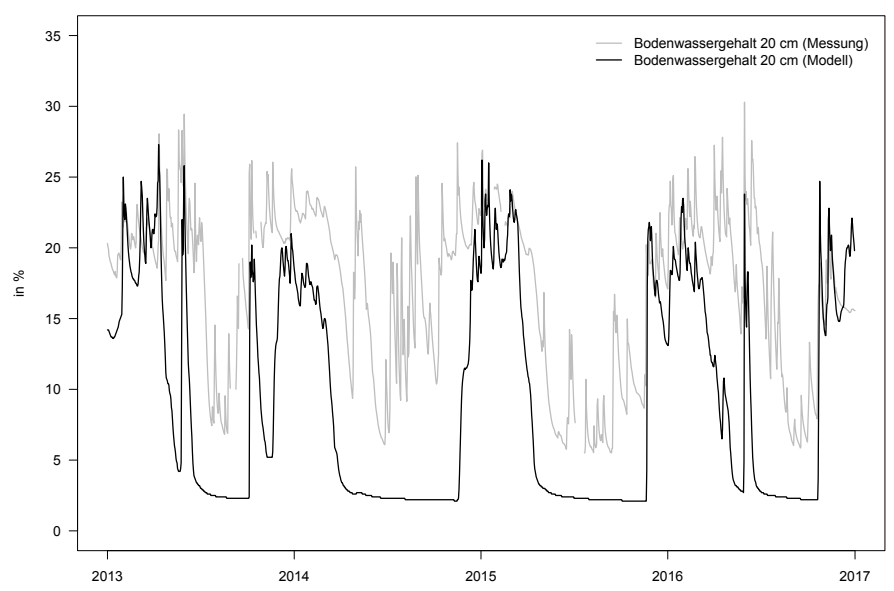

Abbildung 2.13: Vergleich der gemessenen (graue Linie) mit den modellierten (schwarze Linie) Bodenwassergehalten in $20 \mathrm{~cm}$ Bodentiefe der Jahre 2013 - 2016 auf der Level II-Fläche Hessisches Ried.

Eine weitere Validierung fand mit auf den Versuchsparzellen gravimetrisch ermittelten Wassergehalten in 20 und $90 \mathrm{~cm}$ Bodentiefe statt (siehe Abb. 2.14). Die Wassergehalte in $90 \mathrm{~cm}$ Bodentiefe werden durch das Modell etwas überschätzt, während die Bodenwassergehalte in 20 $\mathrm{cm}$ gut abgeschätzt werden.

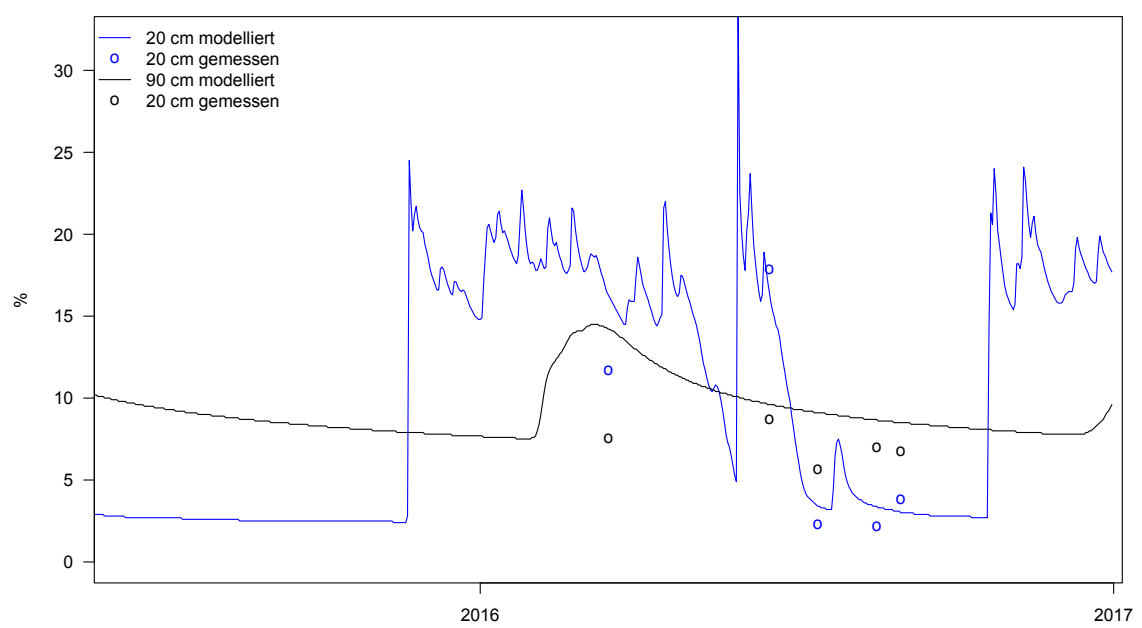

Abbildung 2.14: Vergleich der gemessenen (Kreise) mit den modellierten (Linien) Bodenwassergehalten in $20 \mathrm{~cm}$ (blau) und $100 \mathrm{~cm}$ (schwarz) Bodentiefe der Jahre 2015 und 2016 für die Nullvariante. 


\subsection{Statistik}

Die Daten wurde mit der Statistiksoftware R analysiert (Version 3.4.1). Für die deskriptive Statistik sind häufig Box-Whisker-Plots verwendet worden, welche die Mediane, die 25 bzw. $75 \%$ Perzentile, die oberen und unteren Whisker-Grenzen und Ausreißer enthalten. Die oberen und unteren Whisker-Grenzen stehen für die höchsten bzw. niedrigsten Daten-Werte, welche nicht als Ausreißer definiert werden. Als Ausreißer gelten Messwerte, die außerhalb des 1,5-fachen Interquartilsabstand (75\%-Quartil - $25 \%$-Quartil) liegen, sie werden als Kreise dargestellt.

Die Normalverteilung wurde per Shapiro-Wilk-Test, die Varianzhomogenität der Gruppen mit dem Levene-Test überprüft. Auch die Lage des Medians und die Symmetrie der Boxplots geben Hinweise auf mögliche Abweichungen von der Normalverteilung bzw. Varianzhomogenität.

Die Überprüfung auf Unterschiede zwischen den Varianten fand bei erfüllten Voraussetzungen mittels einfaktorieller ANOVA statt. Sind beide Voraussetzungen nicht erfüllt, so wurde der nichtparametrische Kruskal-Wallis-Test (Rangvarianzanalyse) und bei Signifikanz der multiple Vergleichstest nach Siegel und Castellan (1988) genutzt. Die Prüfung auf Unterschiede in den Überlebensraten erfolgte mittels einem linearen Modell und Binomialverteilung. Das Signifikanzniveau war in allen Tests $\mathrm{p}<0,05$. 


\section{Ergebnisse}

\subsection{Umweltfaktor Trockenheit}

Aus den Klimadaten lässt sich der Zeitraum zu Beginn der Vegetationszeit im Jahr 2014 als Trockenperiode abgrenzen. Die Kohleeinarbeitung und die Pflanzung fällt ebenfalls in diesen Zeitraum. In der Vegetationsperiode 2015 treten mehrere, nur durch minimale Regenereignisse unterbrochene Trockenperioden auf. Dieser Zeitraum kann für die ganze Region als extreme Dürre bezeichnet werden. Zu Beginn des Jahres 2017 fallen nur geringe Niederschläge, sodass die Pflanzen mit einem Niederschlagsdefizit in die Vegetationsperiode starten.

Die per TDR-Sonden gemessene Bodenfeuchte im Oberboden einer Nullparzelle weist mittlere Werte zwischen 2 und $5 \%$ (siehe Abbildung 3.1 (links)) auf. Auch durch größere Niederschlagsereignisse wird der Wassergehalt nur kurzfristig für etwa 2 - 3 Tage angehoben, um danach wieder in den Bereich unter $5 \%$ zu fallen. In der Vegetationsperiode 2015 und zu Beginn der Vegetationszeit 2017 zeigen sich sehr niedrige Wassergehalte in $30 \mathrm{~cm}$ Bodentiefe.

Der Vergleich des volumetrischen Bodenwassergehalts zwischen TDR-Bodenfeuchtesonden und den gravimetrisch ermittelten (in Vol.\% umgerechneten) Bodenwasssergehalten zeigt eine gute Übereinstimmung in den tieferen Bodenschichten. Große Abweichungen bestehen dagegen in der oberen Bodenschicht, hier unterschätzen die TDR-Sonden die Bodenfeuchte um bis zu $15 \%$.
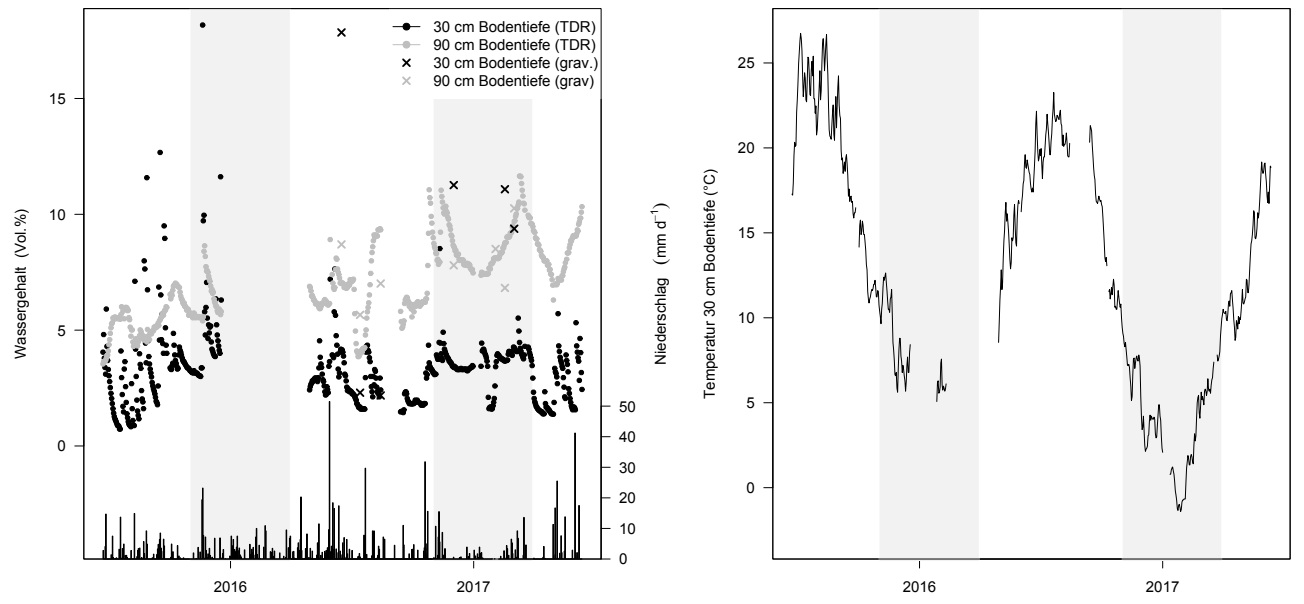

Abbildung 3.1: Bodenwassergehalt (links) und Bodentemperatur (rechts) in $30 \mathrm{~cm}$ Bodentiefe im Zeitraum 23.06.2015 bis 13.06.2017 auf einer Parzelle der Nullvariante. Durch TDR-Sonden und an Feld-Bodenproben gravimetrisch ermittelte Wassergehalte [Vol.\%] in den Bodentiefen $30 \mathrm{~cm}$ (schwarz) und $90 \mathrm{~cm}$ (grau). Tagesniederschlagssumme [mm] der DWD-Station Mannheim

Die Temperatur in $30 \mathrm{~cm}$ Bodentiefe wird durch die Umgebungstemperatur und die Intensität der Solarstrahlung bestimmt und folgt einem saisonalen Verlauf (siehe Abb. 3.1 (rechts)). Im Sommer 2015 treten mittlere Tagestemperaturen von über $25^{\circ} \mathrm{C}$ in $30 \mathrm{~cm}$ Bodentiefe auf. 


\section{2 Änderung der Retentionseigenschaften des Bodens durch den Eintrag von Biokohle}

\subsubsection{Retentionskurven und daraus abgeleitete Parameter}

Die Retentionskurven des Oberbodens $(0-20 \mathrm{~cm})$ weisen eine deutliche Beeinflussung durch die Kohlebeimischung auf (siehe Abb. 3.2 (links)). Die Böden der Kohlevarianten zeigen bei gleichen pF-Stufen höhere Wassergehalte als der durch das sandige Ausgangssubstrat geprägte Boden der Nullvariante (Rechtsverschiebung der Wasserhaltekurven).

Die Wasserspannungskurven für den Unterboden $(30-60 \mathrm{~cm})$ zeigen kaum Unterschiede zwischen den Versuchsvarianten (siehe Abb. 3.2 (rechts)). Die fehlende Kohlebeimischung in diesem Bodenhorizont offenbart das überwiegend sandige Ausgangssubstrat mit seiner geringen Wasserhaltekapazität. Für weitere Berechnungen wird ein homogener Unterboden angenommen und deshalb der jeweilige Mittelwert aus allen Messreihen verwendet.
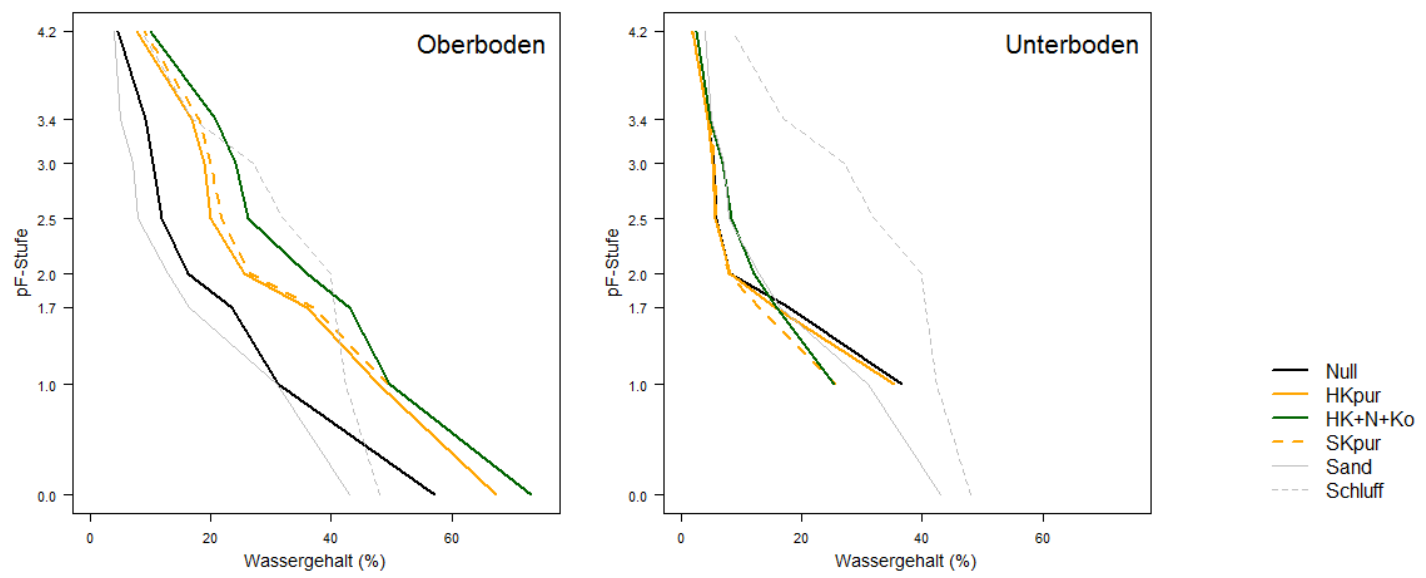

Abbildung 3.2: Retentionskurven des Oberbodens (0 - $20 \mathrm{~cm}$, links) und des Unterbodens (30 $60 \mathrm{~cm}$, rechts) der Versuchsvarianten. Werte für Korngrößengruppen Sand (grau durchgezogene Linie) und Schluff (grau gestrichelte Linie) aus Hartge et al. (2014)

Die nutzbare Feldkapazität (nFK) (siehe Tab. 3.1) zeigt für die Nullvariante im Ober- und Unterboden ähnliche Werte (18,9 bzw. 14,6 Vol.-\%). Die Kohlevarianten weisen im Oberboden um 9,3 Vol.-\% (HKpur) bzw. 14,2 Vol.-\% (HK+N+Ko) höhere nutzbare Feldkapazitäten auf. Die Kohlevariante $\mathrm{HK}+\mathrm{N}+\mathrm{Ko}$ nähert sich in ihren Retentionseigenschaften im Oberboden im pFBereich zwischen 1,7 und 3,4 einem reinen Schluff an.

Für die Berechnung der nutzbaren Feldkapazität wurde der Oberboden von 0 - $25 \mathrm{~cm}$ und der Unterboden von 25 - $90 \mathrm{~cm}$ eingeteilt. Die nFK-Summe des Bodenprofils bis $90 \mathrm{~cm}$ Bodentiefe wird durch die Kohleeinarbeitung in den Oberboden leicht verbessert. Die Erhöhung liegt zwischen 23,2 mm und 35,5 mm (16,3\% bzw. 24,9 \%). Damit ist das Versuchsziel einer Erhöhung der nutzbaren Feldkapazität um etwa 15 Vol.-\% mehr als erfüllt. 
Tabelle 3.1: Wassergehalt bei Feldkapazität $\left(\mathrm{WG}_{\mathrm{FK}}\right)$ und permanentem Welkepunkt (WGPWP) und nutzbare Feldkapazität (nFK) in Ober- (oben, $0-20 \mathrm{~cm}$ ) und Unterboden (unten, $30-60 \mathrm{~cm}$ ) und Profilsumme der nFK bis in $90 \mathrm{~cm}$ Bodentiefe in $\mathrm{mm}$

\begin{tabular}{cccccccc}
\hline \multirow{2}{*}{ Variante } & \multicolumn{2}{c}{$\mathrm{WG}_{\mathrm{FK}}[\%]$} & \multicolumn{2}{c}{$\mathrm{WG}_{\mathrm{PWP}}[\%]$} & \multicolumn{2}{c}{$\mathrm{nFK}[\%]$} & \multirow{2}{*}{ nFK-Summe bis $90 \mathrm{~cm} \mathrm{[mm]}$} \\
& oben & unten & oben & unten & oben & unten & \\
Null & 23,4 & & 4,5 & & 18,9 & & 142,2 \\
HKpur & 36,0 & 16,5 & 7,8 & 1,9 & 28,2 & 14,6 & 165,4 \\
HK+N+Ko & 43,1 & 10,0 & 33,1 & 177,7 \\
Sand & 16,5 & \multicolumn{2}{c}{4,0} & 12,5 & 112,5 \\
Schluff & 40,5 & \multicolumn{2}{c}{8,5} & 32,0 & 288 \\
\hline
\end{tabular}

\subsubsection{Gravimetrischer Bodenwassergehalt in Ober- und Unterboden}

Die gravimetrischen Wassergehalte wurden in volumetrische Wassergehalte umgerechnet und auf die Werte der Nullvariante normiert (Nullvariante entspricht $100 \%$ ). Sie zeigen im Oberboden für die Kohlevarianten deutlich höhere Werte als für die Nullvariante (siehe Tab. 3.2 und Abb. A.2 (links)).

Tabelle 3.2: Mittlere volumetrische Bodenwassergehalte der Kohlevarianten normiert auf den Bodenwassergehalt der Nullvariante [\%]. Oberboden (10 - $20 \mathrm{~cm}$ Bodentiefe) und Unterboden (80 - $90 \mathrm{~cm}$ Bodentiefe). Mittelwert (Standardabweichung), alle Messtermine, $\mathrm{n}=8$.

\begin{tabular}{ccc}
\hline Variante & Oberboden & Unterboden \\
\hline HKpur & $155,9(108,9)$ & $82,9(29,7)$ \\
SKpur & $183,4(82)$ & $84,2(23,3)$ \\
HK+N & $168,0(61,8)$ & $84,1(23,2)$ \\
SK+N & $145,5(69,3)$ & $88,7(34,8)$ \\
HK+N+Ko & $159,0(85,9)$ & $80,3(26,1)$ \\
SK+N+Ko & $124,0(54,8)$ & $75,8(28,2)$ \\
\hline
\end{tabular}

Im Unterboden in etwa $90 \mathrm{~cm}$ Bodentiefe weisen die Kohlevarianten dagegen meist niedrigere Bodenwassergehalte als die Nullvariante auf (siehe Abb. A.2 (rechts)). In Trockenperioden während der Vegetationszeit ist der Unterschied größer als in den Wintermonaten mit höheren Bodenwassergehalten. Durch eine höhere Wasserspeicherung des kohlebehandelten Oberbodens gelangt weniger Wasser in die tiefergelegenen Bodenhorizonte. Während der Vegetationszeit sind kurze Regenereignisse mit geringen Niederschlagsmengen nicht in der Lage, den Unterboden bis in $90 \mathrm{~cm}$ Tiefe zu durchfeuchten. 


\subsubsection{Trockenrohdichte}

Die in den Jahren 2014, 2015 und 2016 ermittelten Trockenrohdichten weisen einen Tiefengradienten auf (siehe Tab. 3.3). Während die Werte der Trockenrohdichte im Jahr 2015 ein Jahr nach Bodenbearbeitung im Bereich $0-20 \mathrm{~cm}$ unter $1,25 \mathrm{~g} \mathrm{~cm}^{-3}$ liegen und damit sehr geringe Dichten aufweisen, zeigen die unbearbeiteten Bodenschichten $(<30 \mathrm{~cm}$ Bodentiefe) Werte über $1,5 \mathrm{~g} \mathrm{~cm}^{-3}$ und damit mittlere Trockenrohdichten nach Standortskartierung (2003) auf. Die Nullvariante wurde 2014 nach dem Mulchen und einer Bodensetzung von etwa 2 Monaten jedoch vor dem Fräsen des Oberbodens beprobt. Das Fräsen hat den Oberboden stark aufgelockert, dies zeigen die Werte der Trockenrohdichte im Jahresvergleich 2014 und 2015. Es dauert etwa 2 Jahre bis sich der Oberboden wieder gesetzt hat und vergleichbare Trockenrohdichten wie nach dem Mulchvorgang aufweist (siehe Abbildung 3.3). Der Setzungsprozess verlief möglicherweise durch die Trockenperiode im Jahr 2015 mit ausbleibenden Niederschlägen etwas gehemmt.

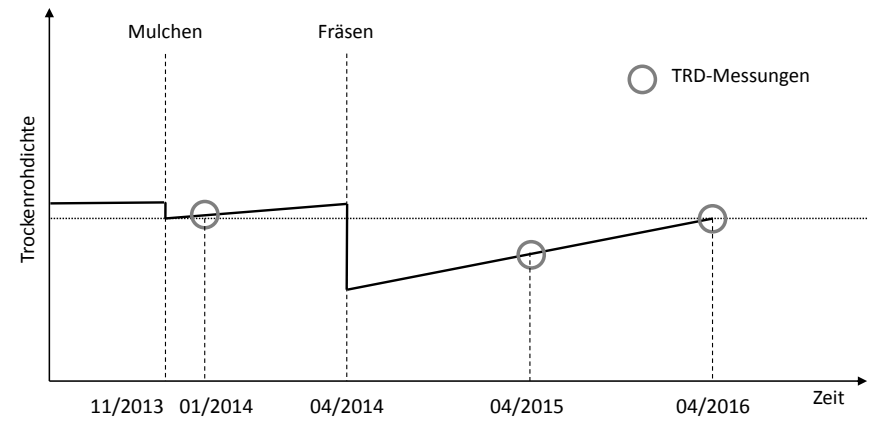

Abbildung 3.3: Schema der Änderung der Trockenrohdichte mit der Bodenbearbeitung

Die Kohle hat einen deutlichen Einfluss auf die Trockenrohdichte des sandigen Substrates. Während die Nullvariante zwei Jahre nach Bodenbearbeitung Werte von über $1,0 \mathrm{~g} \mathrm{~cm}^{-3}$ im Oberboden aufweist, liegen die Kohlevarianten zum Teil deutlich unter $1,0 \mathrm{~g} \mathrm{~cm}^{-3}$ mit Ausnahme der Variante $\mathrm{HK}+\mathrm{N}+\mathrm{Ko}$ in Bodentiefe $10-20 \mathrm{~cm}$. Die pure Spelzenkohlevariante weist in den oberen Bodenschichten bis $30 \mathrm{~cm}$ signifikant geringere Trockenrohdichten als der Oberboden der Nullvariante auf. Die Nullvariante und Variante HK+N+Ko zeigen im Jahresvergleich 2015 und 2016 in fast allen Tiefenstufen ansteigende TRD-Werte und damit Anzeichen einer Bodensetzung. Demgegenüber weisen die puren Kohlen im Oberboden sinkende Werte in der Trockenrohdichte auf. Im Oberboden dieser Varianten hat demzufolge keine Bodensetzung stattgefunden.

Tabelle 3.3: Mittlere Trockenrohdichten in $\mathrm{g} \mathrm{cm}^{-3}$ in den Jahren 2014, 2015 und 2016. Unterschiedliche Buchstaben kennzeichnen signifikante Unterschiede zwischen den Versuchsvarianten in der gleichen Tiefenstufe und im gleichen Jahr $(\mathrm{p}<0,05, \mathrm{n}=3)$.

\begin{tabular}{|c|c|c|c|c|c|c|c|c|c|}
\hline \multirow{2}{*}{ Bodentiefe } & \multicolumn{3}{|c|}{ Null } & \multicolumn{2}{|c|}{ HKpur } & \multicolumn{2}{|c|}{ SKpur } & \multicolumn{2}{|c|}{$\mathrm{HK}+\mathrm{N}+\mathrm{Ko}$} \\
\hline & 2014 & 2015 & 2016 & 2015 & 2016 & 2015 & 2016 & 2015 & 2016 \\
\hline $0-10 \mathrm{~cm}$ & 0,99 & 0,84 & $1,04^{\mathrm{a}}$ & 0,83 & $0,79^{\mathrm{ab}}$ & 0,81 & $0,75^{\mathrm{b}}$ & 0,79 & $0,93^{\mathrm{ab}}$ \\
\hline $10-20 \mathrm{~cm}$ & 1,31 & 1,24 & $1,32^{\mathrm{a}}$ & 0,99 & $0,95^{\mathrm{ab}}$ & 0,93 & $0,82^{\mathrm{b}}$ & 0,83 & $1,03^{\mathrm{ab}}$ \\
\hline $20-30 \mathrm{~cm}$ & 1,37 & 1,44 & $1,46^{\mathrm{a}}$ & 1,5 & $1,32^{\mathrm{ab}}$ & 1,36 & $0,87^{\mathrm{b}}$ & 1,5 & $1,35^{\mathrm{ab}}$ \\
\hline $30-60 \mathrm{~cm}$ & 1,62 & 1,57 & 1,5 & & 1,55 & 1,57 & 1,59 & & 1,53 \\
\hline $60-90 \mathrm{~cm}$ & 1,55 & 1,48 & 1,54 & & 1,5 & 1,53 & 1,54 & & 1,5 \\
\hline
\end{tabular}




\subsubsection{Hydrophobe Bodeneigenschaften}

\subsubsection{Versickerungszeit im Feldversuch}

Die mittlere Versickerungszeit weist während des Sommers 2015 einen stetigen Anstieg auf (siehe Tab. 3.4). Mit fortschreitender Vegetationszeit und Austrocknung des Oberbodens verlängert sich die Versickerungsdauer in allen Versuchsvarianten deutlich. Die Flächen der Nullvariante weisen bereits am ersten Termin sehr hohe Versickerungszeiten von mehr als 3 Minuten pro $100 \mathrm{ml}$ auf. Auch an den Folgeterminen lassen sich auf Flächen dieser Variante die höchsten Versickerungszeiten beobachten. Am ersten Probetermin zeigen die stickstoff- $(+\mathrm{N})$ und kompost$(+\mathrm{N}+\mathrm{Ko})$ nachbehandelten Kohlevarianten deutlich kürzere Versickerungszeiten von unter einer Minute pro $100 \mathrm{ml}$. Die Versickerungszeit der puren Holzkohlevarianten ist an diesem Termin etwa doppelt so lang wie bei den übrigen Kohlevarianten. Am Folgetermin etwa eine Woche später ist dieses Muster nicht mehr zu erkennen.

Tabelle 3.4: Mittlere Versickerungszeit in Sekunden pro $100 \mathrm{ml}$ auf den Parzellen der Versuchsvarianten im Sommer 2015. Mittelwert (Standardabweichung), $\mathrm{n}=3$.

\begin{tabular}{cccc}
\hline Variante & 30.06. & 07.07. & 14.07. \\
\hline Null & $196(100)$ & $256(26)$ & $280(0)$ \\
HKpur & $117(57)$ & $132(24)$ & $249(54)$ \\
SKpur & $109(115)$ & $163(104)$ & $224(64)$ \\
HK+N & $53(42)$ & $225(53)$ & $236(77)$ \\
SK+N & $54(46)$ & $171(95)$ & $270(18)$ \\
HK+N+Ko & $53(39)$ & $199(117)$ & $262(32)$ \\
SK+N+Ko & $43(20)$ & $245(60)$ & $280(0)$ \\
\hline
\end{tabular}

\subsubsection{Aufsättigungszeit im Topfversuch}

Im Topfversuch zeigte Variante SK+N ein vergleichsweise langsames Aufsättigungsverhalten (siehe Tab. 3.5). Auch nach 14 Tagen kontinuierlicher Aufsättigung mit teilweisem Besprühen der Bodenoberfläche konnte bei über 50 \% der Töpfe dieser Variante keine volle Aufsättigung erreicht werden. Im Vergleich zur Kontrolle hat die Zugabe von Spelzenkohle der Varianten SK $+\mathrm{N}$ und $\mathrm{SK}+\mathrm{N}+\mathrm{Ko}$ eine verzögerte, die Zugabe von Holzkohle der Varianten HKpur und HK+N (Py) eine beschleunigte Aufsättigung zur Folge.

Tabelle 3.5: Anzahl der wassergesättigten Töpfe in der Vorbereitung des Topfversuches, $\mathrm{n}=8$.

\begin{tabular}{cccc}
\hline Variante & 3. Mai 2017 & 4. Mai 2017 & 10. Mai 2017 \\
\hline Null & 3 & 7 & 7 \\
HKpur & 6 & 7 & 8 \\
SKpur & 3 & 6 & 6 \\
HK+N (Py) & 7 & 8 & 8 \\
HK+N (Se) & 2 & 7 & 7 \\
SK+N & 2 & 2 & 3 \\
HK+N+Ko & 2 & 7 & 8 \\
SK+N+Ko & 1 & 4 & 6 \\
\hline
\end{tabular}




\subsubsection{Sickerwassermodellierung}

Ein großer Teil der Grundwasserspende findet in der Nichtvegetationszeit in den Monaten Januar bis April statt (siehe Abb. 3.4). Als Nachwirkung des Trockenjahres 2015 zeigt sich im Jahr 2016 ein verzögerter Beginn des Sickerwasserflusses erst im Februar (Nullvariante) bzw. März (Kohlevarianten).

Die Sickerwassermengen der Kohlevarianten HKpur und $\mathrm{HK}+\mathrm{N}+\mathrm{Ko}$ unterscheiden sich nur wenig voneinander. Beide Kohlevarianten zeigen aber deutlich reduzierte Sickerwassermengen im Vergleich zur Nullvariante. In der Zeit der größten Grundwasserspende unterscheiden sich die Mengen zwischen den Versuchsvarianten kaum, die unterschiedlichen Jahresmengen ergeben sich vor allem aus dem späteren Beginn und frühzeitigeren Ende des Sickerwasseraufkommens.

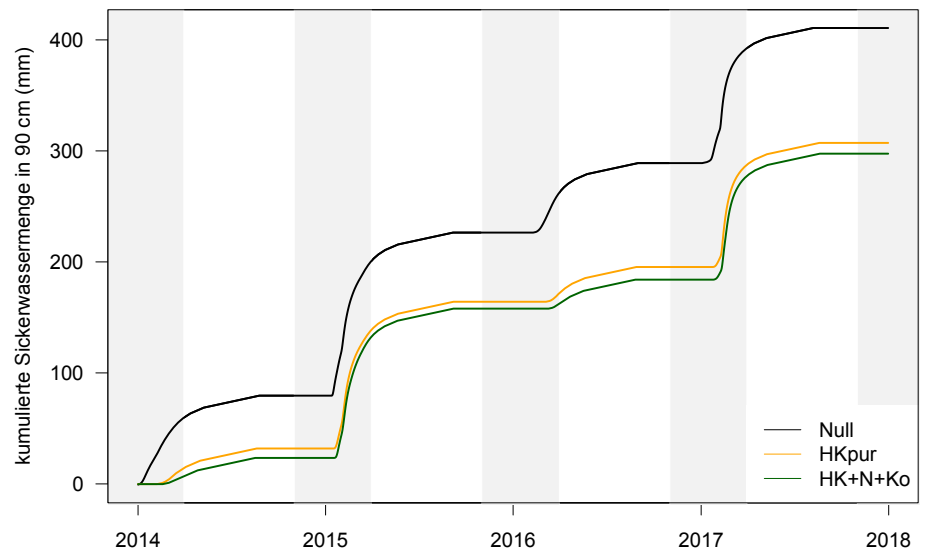

Abbildung 3.4: Kumulierte Sickerwassermengen in mm der Jahre 2014 - 2018. 


\subsection{Umweltfaktor Stickstoff}

In diesem Abschnitt soll der Umweltfaktor bzw. Stressor Stickstoffverfügbarkeit näher quantifiziert werden. Dabei soll der Schwerpunkt auf den Stickstoffeintrag, die Vorratsänderungen und die Austräge mit dem Sickerwasser gelegt werden. Zusammen mit weiteren beschreibenden Faktoren wie der Dynamik und dem Artenaufkommen in der Begleitvegetation bilden sie die Grundlage für eine Stickstoffbilanzierung innerhalb des Versuchszeitraumes.

\subsubsection{Stickstoffeinträge}

Die Gesamtstickstoffeinträge durch die Kohleeinarbeitung ergeben sich aus den Stickstoffgehalten der Kohlevarianten und der Gesamteintragsmenge von $80 \mathrm{t} \mathrm{ha}^{-1}$ (siehe Tab. 2.7 und Tab. 3.6).

Tabelle 3.6: Gesamtstickstoffgehalte der Kohlen $\left[\mathrm{g} \mathrm{kg}^{-1}\right]$, Eintragsmengen $\left[\mathrm{kg} \mathrm{ha}^{-1}\right]$ und Anteil des Eintrages am ursprünglichen Stickstoffvorrat 2014 bis $90 \mathrm{~cm}$ Bodentiefe [\%]

\begin{tabular}{cccc}
\hline Variante & $\begin{array}{c}\mathrm{N}_{\text {ges }} \text {-Gehalt } \\
\mathrm{g} \mathrm{kg}^{-1}\end{array}$ & $\begin{array}{c}\mathrm{N}_{\text {ges }} \text {-Eintrag } \\
\mathrm{kg} \mathrm{ha}^{-1}\end{array}$ & $\begin{array}{c}\mathrm{N}_{\text {ges }} \text {-Eintrag/Vorrat 2014 } \\
\%\end{array}$ \\
\hline HKpur & 3,6 & 289 & 8,03 \\
SKpur & 12,3 & 986 & 27,39 \\
HK+N (Py) & 11,5 & 921 & 25,58 \\
HK+N (Se) & 7,8 & 625 & 17,36 \\
SK+N & 12,1 & 969 & 26,92 \\
HK+N+Ko & 11,1 & 890 & 24,72 \\
SK+N+Ko & 14,2 & 1133 & 31,47 \\
\hline
\end{tabular}

Der Stickstoffvorrat erhöht sich durch die Kohleneinarbeitung zwischen $8 \%$ und $31 \%$. Die Einbringung von Spelzenkohle (SK) hat im Vergleich zu Holzkohle (HK) einen deutlich höheren Stickstoffeintrag zur Folge. Der Nchbehandlungsprozess der Kompostierung führt zu einer abermaligen Erhöhung der Stickstoffeinträge.

Die jährliche luftbürtige Stickstoffdeposition zwischen 1999 und 2016 beträgt an der etwa $5 \mathrm{~km}$ entfernten Level II-Fläche Hessisches Ried (Freiland) etwa 6,91 $\mathrm{kg} \mathrm{ha}^{-1} \mathrm{a}^{-1}$. Für den Zeitraum 2013 - 2015 gibt das Umweltbundesamt am Versuchsstandort eine Stickstoffdeposition von $10 \mathrm{~kg}$ $\mathrm{ha}^{-1} \mathrm{a}^{-1}$ an (Schaap et al. 2015).

\subsubsection{Stickstoff- und Kohlenstoffvorrat im Boden}

Der Kohlenstoffeintrag mit der Kohle beträgt je nach Variante zwischen 28 und 69 t ha $^{-1}$ (vgl. Tabelle 2.7). Dementsprechend ist bei den Kohlevarianten, gegenüber der Nullvariante ein um diesen Betrag höherer Kohlenstoffvorrat zu erwarten (siehe Abb. 3.5 (links)). Dies trifft nur für die kompostnachbehandelten $(+\mathrm{N}+\mathrm{Ko})$ Kohlevarianten $\mathrm{zu}$, wobei die hohe Streuung der Werte innerhalb der Varianten zu berücksichtigen ist. Ein Erklärungsansatz kann die Vorbehandlung der Bodenproben sein. Die Proben wurden mit einem Sieb von $2 \mathrm{~mm}$ Maschenweite gesiebt. Als grober Bodenbestandteil wird die Kohle zu einem gewissen Teil ausgesiebt und gelangt somit nicht vollständig in die chemische Analyse des Feinbodens. Dies hat ebenso Auswirkungen auf den Stickstoffvorrat, da ein Großteil des eingetragenen Stickstoffes durch die Kohlepartikel dauerhaft adsorbiert wird (siehe Abb. 3.5 (rechts)).

Die Variante HK $+\mathrm{N}$ weist die geringsten Stickstoffvorräte auf. Dieser Befund ist angesichts der 
hohen Stickstoffeinträge in dieser Variante unerwartet. Hier besteht aber auch eine große Abweichung zum rechnerischen Kohlenstoffvorrat und spricht für die These der unvollständigen Eingang der Kohlen in die chemische Analyse. Lediglich die Kompostvarianten $\mathrm{HK}+\mathrm{N}+\mathrm{Ko}$ und $\mathrm{SK}+\mathrm{N}+$ Ko zeigen Stickstoffvorräte von über $4 \mathrm{t} \mathrm{ha}^{-1}$ und damit Werte nahe an den rechnerischen Einträgen.
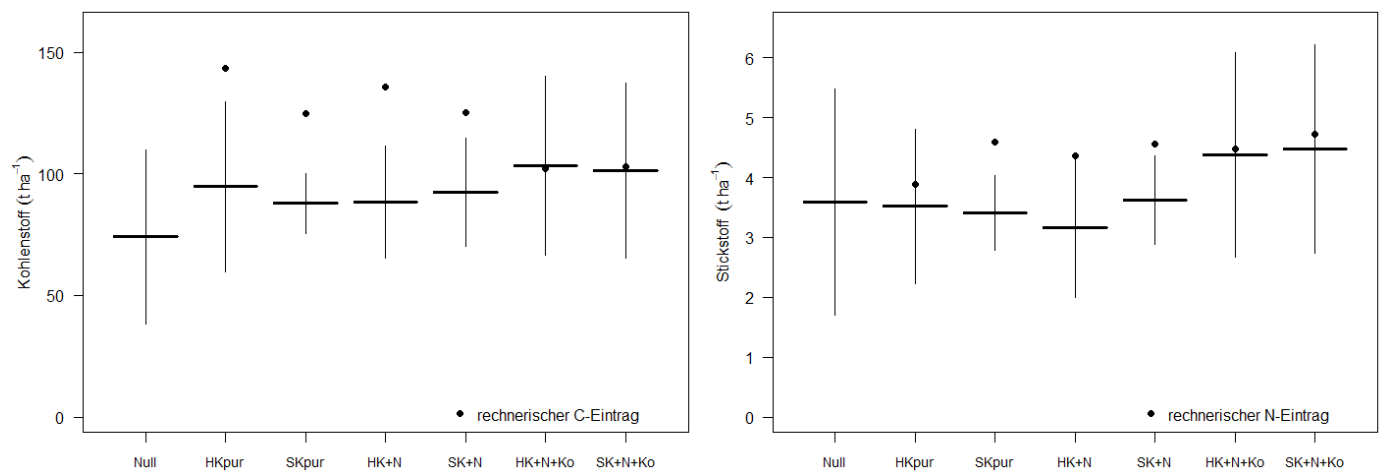

Abbildung 3.5: Kohlenstoff- (links) und Stickstoffvorräte (rechts) im Oberboden im Jahr 2015 (Mittelwert und Standardabweichung), $\mathrm{n}=6$. Punkte stellen den rechnerischen Kohlenstoff- (C) bzw. Stickstoffeintrag (N) bei $80 \mathrm{t} \mathrm{ha}^{-1}$ Kohleneintrag dar.

\subsubsection{Stickstoffaustrag}

\subsubsection{Stickstoffaustrag mit dem Sickerwasser im Feldversuch}

Die Konzentrationen der in $90 \mathrm{~cm}$ Bodentiefe gewonnen Bodenlösung zeichnen die Stoffeinträge der Versuchsvarianten gut nach (siehe Abbildung 3.6). Die Konzentrationen von Nitrat und Ammonium in der Bodenlösung nehmen in allen Varianten von Anfang 2015 bis Ende 2017 deutlich ab.

Die Nitrat-N-Konzentrationen liegen im Jahr 2015, ein Jahr nach Kohleneinarbeitung, bei den stickstoff- $(+\mathrm{N})$ und kompost- $(+\mathrm{N}+\mathrm{Ko})$ nachbehandelten Varianten $(\mathrm{HK}+\mathrm{N}, \mathrm{SK}+\mathrm{N}, \mathrm{HK}+\mathrm{N}+\mathrm{Ko}$ und $\mathrm{SK}+\mathrm{N}+\mathrm{Ko}$ ) über dem Grenzwert von $50 \mathrm{mg} \mathrm{l}^{-1}$, der durch die EG-Wasserrahmenrichtlinie als Norm für die Grundwasserqualität formuliert wird (siehe Abb. 3.6 (links)). Die Variante HK $+\mathrm{N}$ liegt auch im Jahr 2016 noch über diesem Grenzwert. Die Nullvariante zeigt im Jahr 2015 während der Vegetationszeit mit deutlichem Abstand die geringsten Nitratkonzentrationen. 

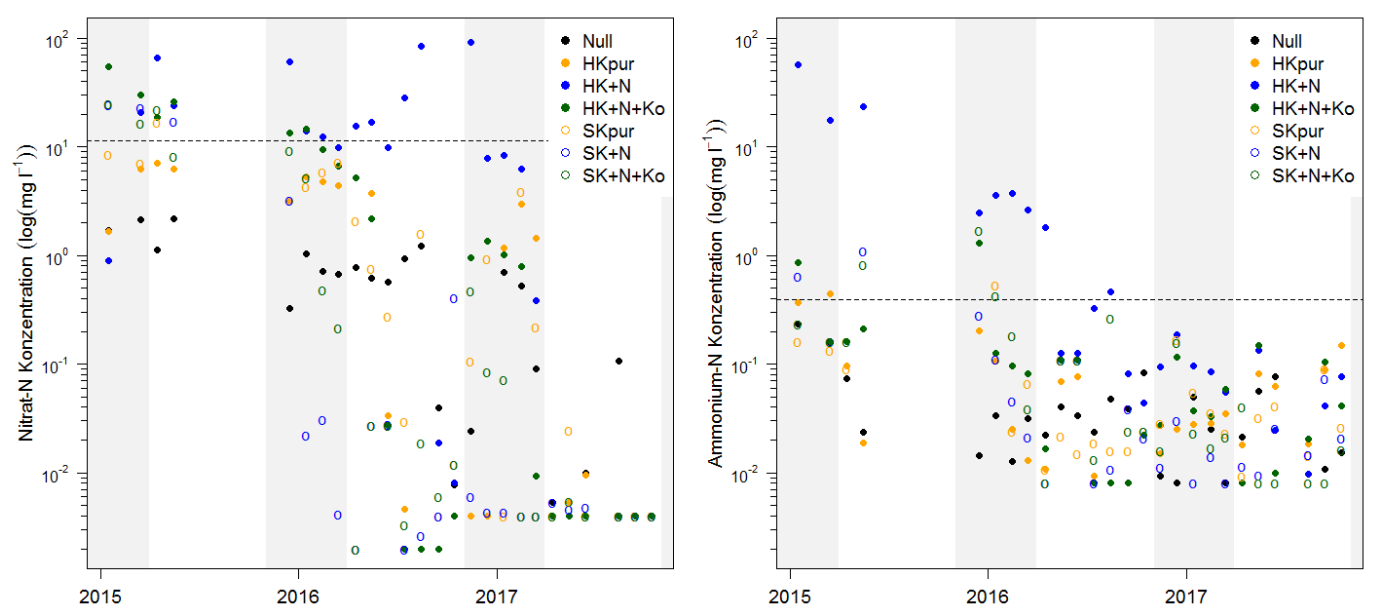

Abbildung 3.6: Logarithmische Darstellung der mittleren Nitrat-N- (links) und Ammonium-NKonzentration (rechts) des Sickerwassers in den Jahren 2015 - 2017. Grenzwerte für Einleitung in das Grundwasser entsprechen einer Nitratkonzentration von 50 $\mathrm{mg} \mathrm{l}^{-1}$ bzw. einer Ammoniumkonzentration von $0,5 \mathrm{mg} \mathrm{l}^{-1}$ (gestrichelte schwarze Linien). Die Nichtvegetationszeiten (1. November - 31. März) sind grau hinterlegt, $\mathrm{n}=3$.

Abbildung 3.7 zeigt die Nitrat- und Ammonium-Stickstoffausträge der Nullvariante und jeweils die Differenz zwischen der entsprechenden Kohlevariante und der Nullvariante während der Vegetationszeiten (VZ) und Nichtvegetationszeiten (NVZ).

Durch den Stickstoffbedarf der Pflanzen vor allem zu Beginn der Vegetationszeit weist die Bodenlösung in ungestörten Ökosystemen während der Vegetationszeit niedrigere Stickstoffgehalte als in der Nichtvegetationszeit auf. Die zum Teil sehr hohen Stickstoffeinträge und die durch Bodenbearbeitung verursachten Mineralisationsprozesse überprägen dieses saisonale Reaktionsmuster in der ersten Phase des Feldversuches bis Ende 2015. Ab der Vegetationszeit 2016 lässt sich bei der Nullvariante und den puren Kohlevarianten (HKpur und SKpur) das beschriebene Muster erkennen. Bei diesen Varianten steigen in den Herbstmonaten des Jahres 2016 die Nitratkonzentrationen im Sickerwasser aufgrund der abnehmenden Stickstoffaufnahme durch die Pflanzen und der einsetzenden Mineralisierung abgestorbener Pflanzenteile (Blätter und Begleitvegetation) an. Die stickstoff- $(+\mathrm{N})$ und kompost- $(+\mathrm{N}+\mathrm{Ko})$ nachbehandelten Varianten zeigen während der gesamten Untersuchungsdauer kaum saisonale Reaktionsmuster. Besonders Variante HK+N weist noch bis Ende 2016 einen anhaltend hohen Nitrataustrag auf, der keinem saisonalen Verlauf folgt.

Ab 2016 liegen die Nitratkonzentrationen der stickstoff- $(+\mathrm{N})$ und kompost- $(+\mathrm{N}+\mathrm{Ko})$ nachbehandelten Kohlevarianten (mit Ausnahme von $\mathrm{HK}+\mathrm{N}$ ) ganzjährig unter denen der Nullvariante. Mit zunehmender Verweildauer im Boden scheint das Vermögen der Kohlen Nitrat zu binden eine größere Wirkung zu entfalten. Dieses Immoblisierungspotential überprägt den saisonalen Verlauf der Stickstoffausträge in der zweiten Phase des Feldversuches (ab 2016).

Die puren Kohlevarianten HKpur und SKpur weisen Unterschiede in der Dynamik und Stärke der Stickstofffreisetzung auf. Während die Variante SKpur im Nitrataustrag 2015 noch deutlich über der Variante HKpur liegt, weisen beide puren Kohlesubstrate ab dem Jahr 2016 in etwa gleiche Nitratausträge auf. 

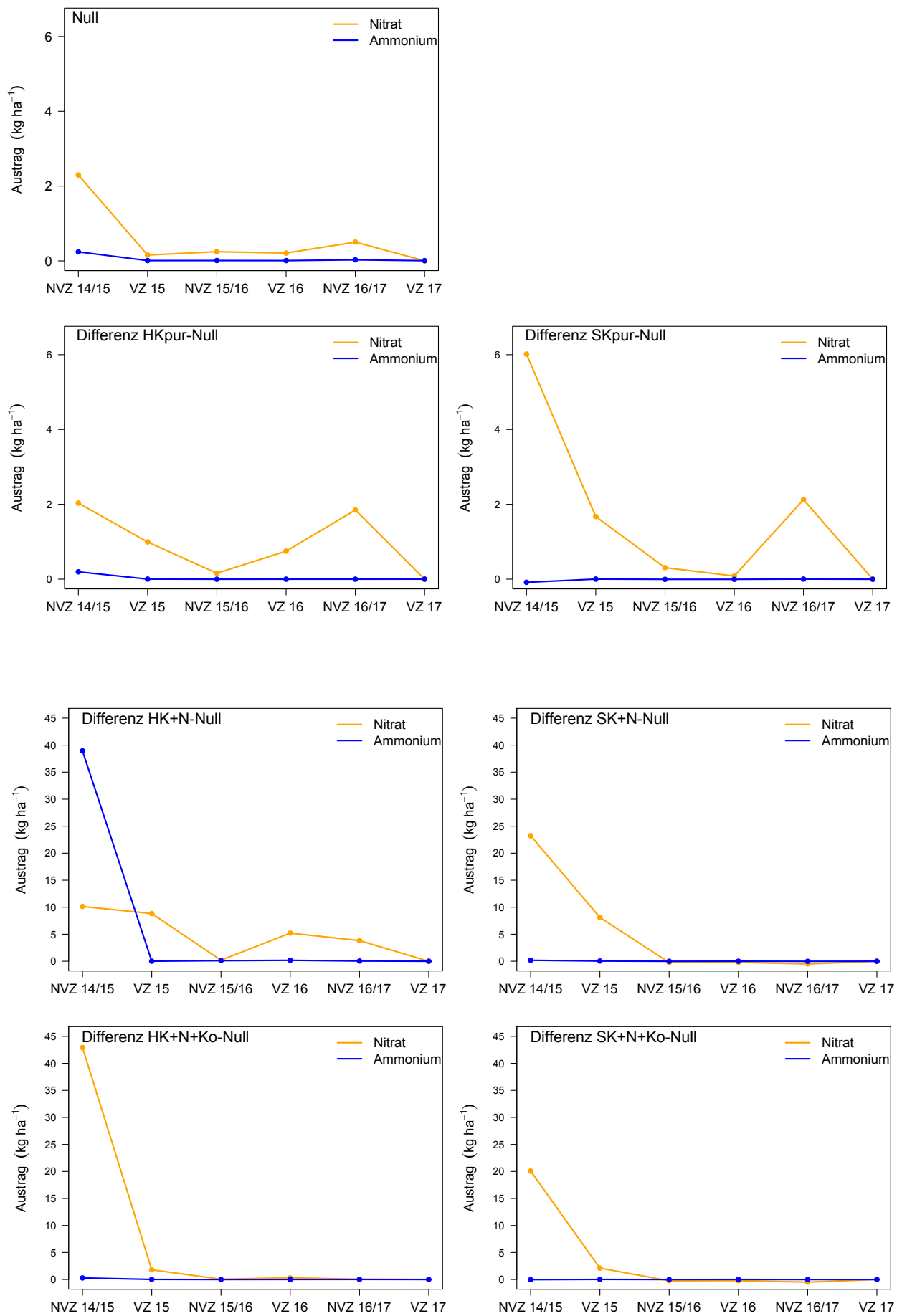

Abbildung 3.7: Mittelwerte der Nitrat- und Ammoniumausträge während der Vegetationszeiten (VZ) und Nichtvegetationszeiten (NVZ) der Jahre 2015 - 2017. Nullvariante und jeweils die Differenzen der Kohlevarianten zur Nullvariante, $n=3$. 
Grundsätzlich sind für Ammonium (mit Ausnahme von Variante HK+N in der Nichtvegetationszeit 2014/15) um den Faktor 10 niedrigere Austräge als für Nitrat zu beobachten (siehe Abb. 3.6 (rechts)).

2015 liegt die Variante HKpur in der Ammonium-N-Konzentration der Bodenlösung leicht über der Variante SKpur, im Folgejahr kehrt sich die Reihenfolge um. 2017 liegen beide Kohlevarianten auf gleichem geringen Niveau. Die Ammonium-N-Konzentration der Variante $\mathrm{HK}+\mathrm{N}$ liegt bis Mitte 2016 über dem Grenzwert für Einleitungen ins Grundwasser von $0,5 \mathrm{mg} \mathrm{l}^{-1}$. Dies betrifft vor allem die Parzelle 2/2, in der Kohle des Herstellers Pyreg eingearbeitet worden ist. Die anfänglich hohen Ammoniumkonzentrationen der Varianten $\mathrm{HK}+\mathrm{N}+\mathrm{Ko}$ und $\mathrm{SK}+\mathrm{N}+\mathrm{Ko}$ gehen im Jahr 2016 stetig zurück. Im Jahr 2017 liegen alle Varianten unter dem Grenzwert.

Tabelle 3.7 zeigt die Jahressummen der Nitrat- und Ammoniumausträge für alle Versuchsvarianten. Alle Versuchsvarianten weisen in den Jahren 2016 und 2017 deutlich geringere Austräge als noch im Jahr 2015 auf. Durch die höheren Sickerwassermengen im Jahr 2017 liegen die Austragsmengen an Nitrat über denen des Jahres 2016 mit Ausnahme der stickstoff- $(+\mathrm{N})$ und kompost- $(+\mathrm{N}+\mathrm{Ko})$ behandelten Kohlevarianten. Hier kompensieren die anhaltend sinkenden $\mathrm{Ni}-$ tratkonzentrationen zwischen beiden Jahren die höheren Sickerwassermengen im Jahr 2017. Die Dynamik des Ammoniumaustrages weist grundsätzlich das gleiche Muster auf, ist jedoch weniger stark ausgeprägt, mit Ausnahme von Variante $\mathrm{HK}+\mathrm{N}$.

Tabelle 3.7: Jahressummen der Nitrat-N und Ammonium-N-Austräge in kg ha ${ }^{-1} 2015$ - 2017.

\begin{tabular}{ccccccc}
\hline \multirow{2}{*}{ Variante } & \multicolumn{2}{c}{ Nitrat-N } & $\left(\mathrm{kg} \mathrm{ha}^{-1}\right)$ & \multicolumn{3}{c}{ Ammonium-N $\left(\mathrm{kg} \mathrm{ha}^{-1}\right)$} \\
& 2015 & 2016 & 2017 & 2015 & 2016 & 2017 \\
\hline Null & 2,454 & 0,456 & 0,505 & 0,251 & 0,018 & 0,034 \\
HKpur & 5,48 & 1,363 & 2,351 & 0,449 & 0,016 & 0,035 \\
SKpur & 10,143 & 0,848 & 2,63 & 0,168 & 0,009 & 0,034 \\
HK+N & 21,423 & 5,861 & 4,332 & 39,202 & 0,298 & 0,079 \\
SK+N & 33,797 & 0,002 & 0,005 & 0,465 & 0,011 & 0,014 \\
HK+N+Ko & 47,18 & 0,82 & 0,545 & 0,551 & 0,014 & 0,045 \\
SK+N+Ko & 24,63 & 0,013 & 0,005 & 0,233 & 0,018 & 0,021 \\
\hline
\end{tabular}

\subsubsection{Stickstoffaustrag mit dem Sickerwasser im Topfversuch}

Im Topfversuch bauen sich die Nitratkonzentrationen im Sickerwasser langsam auf (siehe Abb. 3.8). Nach 26 Tagen liegen die Nitratausträge der Varianten $\mathrm{HK}+\mathrm{N}+\mathrm{Ko}$ und $\mathrm{SK}+\mathrm{N}+\mathrm{Ko}$ über dem Nitratgehalt des Gießwassers. Es findet dementsprechend ein Netto-Nitrataustrag statt. Die übrigen Varianten nehmen Nitrat aus dem Gießwasser auf, wobei die Nullvariante mit dem stickstoffarmen Substrat die höchste Aufnahmerate aufweist. Nach 41 Tagen zeigen die puren Kohlenvarianten (HKpur und SKpur) einen deutlich geringeren Nitrataustrag als die Nullvariante. Die puren Kohlenvarianten nehmen weiterhin Nitrat aus dem Gießwasser auf. Die stickstoff- $(+\mathrm{N})$ und kompost- $(+\mathrm{N}+\mathrm{Ko})$ nachbehandelten Kohlevarianten zeigen mit fortschreitender Versuchsdauer deutliche Nitratausträge. Die Aufnahme von Nitrat durch die Pflanzen kann mit der Nitratfreisetzung dieser Varianten nicht Schritt halten.

Es bestehen Unterschiede in den Nitratgehalten des Sickerwassers zwischen den Baumarten Hainbuche und Kiefer. Zu Beginn zeigen die Varianten SKpur, HK+N (beide) und Null bei der Kiefer geringere Nitratausträge als bei der Hainbuche. An den folgenden Terminen zeigen alle Varianten bei der Kiefer niedrigere Nitratausträge als die entsprechenden Varianten der Hainbuche. Es ist davon auszugehen, das die Kiefern im Topfversuch mehr Nitrat aufnehmen als die Hainbuchen. 

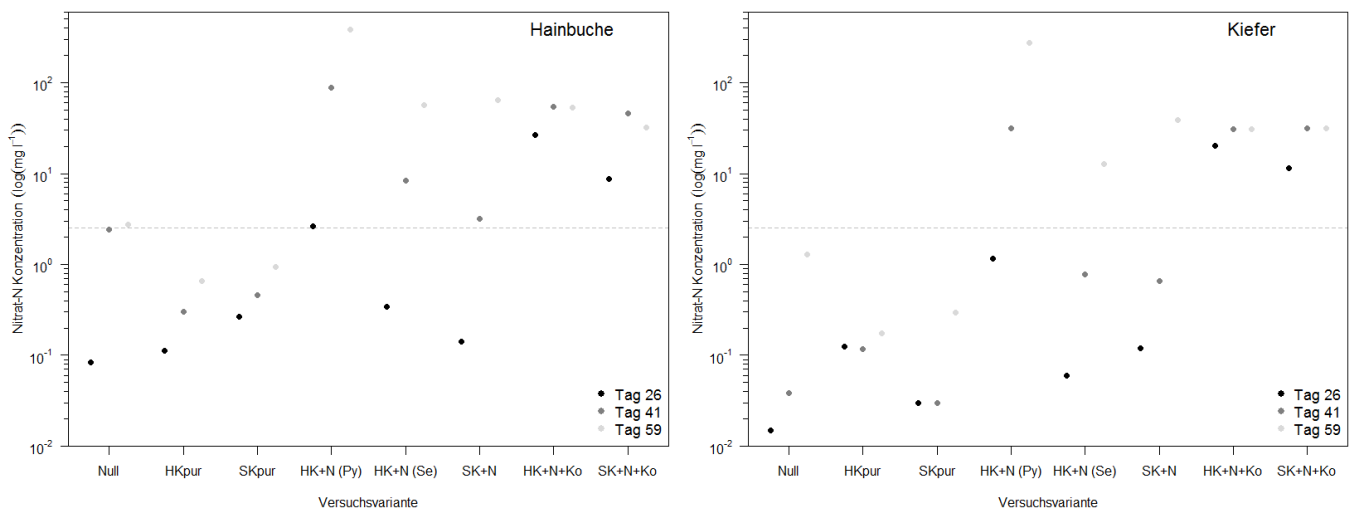

Abbildung 3.8: Mittlerer Nitratgehalt des Sickerwassers im Topfversuch, $n=4$. Gestrichelte Linie entspricht dem Nitratgehalt des Gießwassers. links: Hainbuche, rechts: Kiefer

Die Ammoniumkonzentrationen beider Baumarten zeigen zwischen den Messterminen weniger Dynamik als die Nitratkonzentrationen (siehe Abb. 3.9). Die Ammoniumkonzentrationen aller Versuchsvarianten liegen über dem Ammoniumgehalt des Gießwassers. Für die stickstofffreien puren Kohlenvarianten (HKpur bzw. SKpur) und die Nullvariante lässt sich feststellen, dass der Betrag der Ammonium-Pflanzenaufnahme und der Ammoniumimmobilisierung durch das Kohlesubstrat gering zu sein scheint. Es findet ein Netto-Ammoniumaustrag statt, der bei diesen Varianten auf die Mineralisierung der organischer Substanz zurückzuführen ist.

Die stickstoffbehandelten Kohlevarianten $(+\mathrm{N})$ zeigen an allen Terminen deutlich höhere Ammoniumkonzentrationen als die übrigen Versuchsvarianten (zum Teil die 100fache Konzentration). Spitzenreiter ist die Variante $\mathrm{HK}+\mathrm{N}(\mathrm{Py})$. Die Varianten $\mathrm{HK}+\mathrm{N}+\mathrm{Ko}$ und $\mathrm{SK}+\mathrm{N}+\mathrm{Ko}$ liegen im Ammoniumaustrag etwas über den puren Kohlensubstraten und der Nullvariante.

Die Kiefernvarianten weisen höhere Ammoniumausträge als die entsprechenden Hainbuchenvarianten mit dem Sickerwasser auf. Dies deutet auf eine im Vergleich höhere Ammoniumaufnahme der Hainbuchen. Einzig die Variante HK+N+Ko zeigt kaum Unterschiede zwischen den beiden Baumarten.
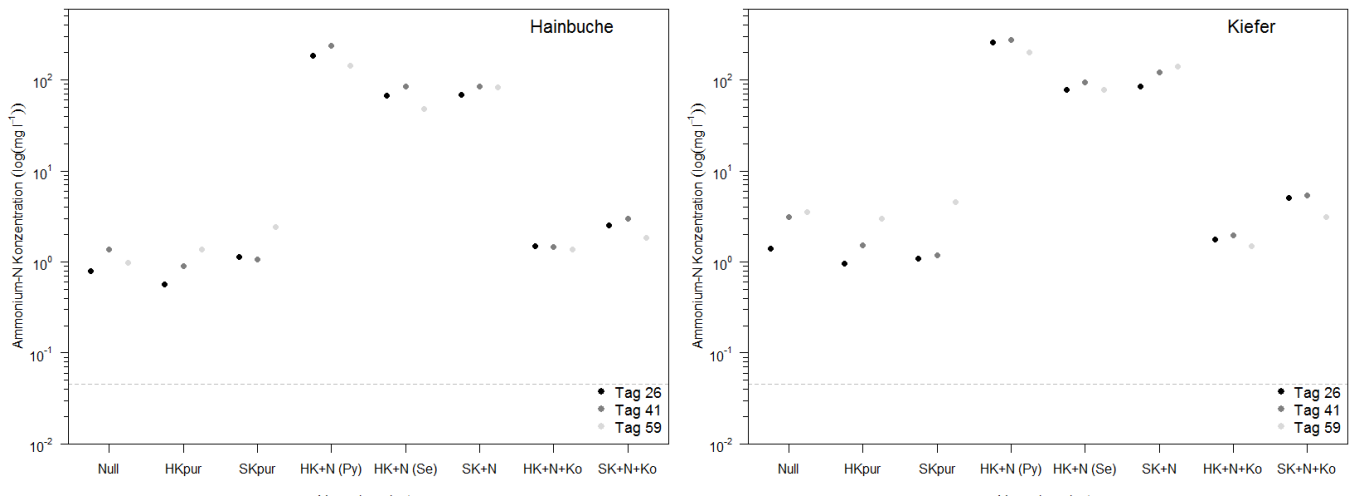

Abbildung 3.9: Mittlerer Ammoniumgehalt des Sickerwassers im Topfversuch, $\mathrm{n}=4$. Gestrichelte Linie entspricht dem Ammoniumgehalt des Gießwassers. links: Hainbuche, rechts: Kiefer 
Die Analyseergebnisse des Sickerwassers zeigen für beide Versuche die gleichen Muster des mineralischen Stickstoffaustrags (siehe Tab. 3.8). Die Varianten HK+N (beide Hersteller), SK $+\mathrm{N}$ und $\mathrm{HK}+\mathrm{N}+\mathrm{Ko}$ nehmen bei den prozentualen Anteilen des Stickstoffaustrages vom -eintrag die Spitzenpostionen ein, während die Variantee SK+N+Ko nur wenig vom eingebrachten Stickstoff austrägt.

Tabelle 3.8: Anteil der mineralischen Stickstoffausträge am Stickstoffeintrag der Versuchsvarianten über das Sickerwasser im Feld- und Topfversuch (\%)

\begin{tabular}{ccc}
\hline Variante & Feldversuch & Topfversuch \\
\hline HKpur & 3,25 & 0,25 \\
SKpur & 1,4 & 0,08 \\
HK+N (Py) & 9,21 & 18,59 \\
HK+N (Se) & 3,54 & 6,35 \\
SK+N & 4,41 \\
HK+N+Ko & 5,52 & 2,42 \\
SK+N+Ko & 2,2 & 1,28 \\
\hline
\end{tabular}




\subsubsection{Stickstoffmineralisation (Extraktionsversuch)}

Um einen genaueren Einblick in die Dynamik der Stickstoffmineralisation während der Initialphase des Versuches zu erhalten, wurde ein Extraktionsversuch unter Laborbedingungen durchgeführt, bei dem an jedem Extraktionstermin der gesamte mineralische Sticktoff ausgespült wurde. Die Höhe des Stickstoffgehaltes im Extrakt spiegelt das Mineralisationspotenzial der Bodenbiologie während des Zeitraumes seit der letzten Extraktion wider.

Nach anfänglich sehr hohen Spitzenwerten sinken die Nitrat-N-Konzentrationen in allen Versuchsvarianten ab (siehe Abb. 3.10 (links)). Dieser initiale Spitzenwert könnte durch hohe Mengen mineralisierten Stickstoffs an den zwei Jahre alten Rückstellproben der Kohlen verursacht worden sein. Diese hohen Mengen konnten möglicherweise bei der ersten Extraktion nicht vollständig ausgewaschen werden.
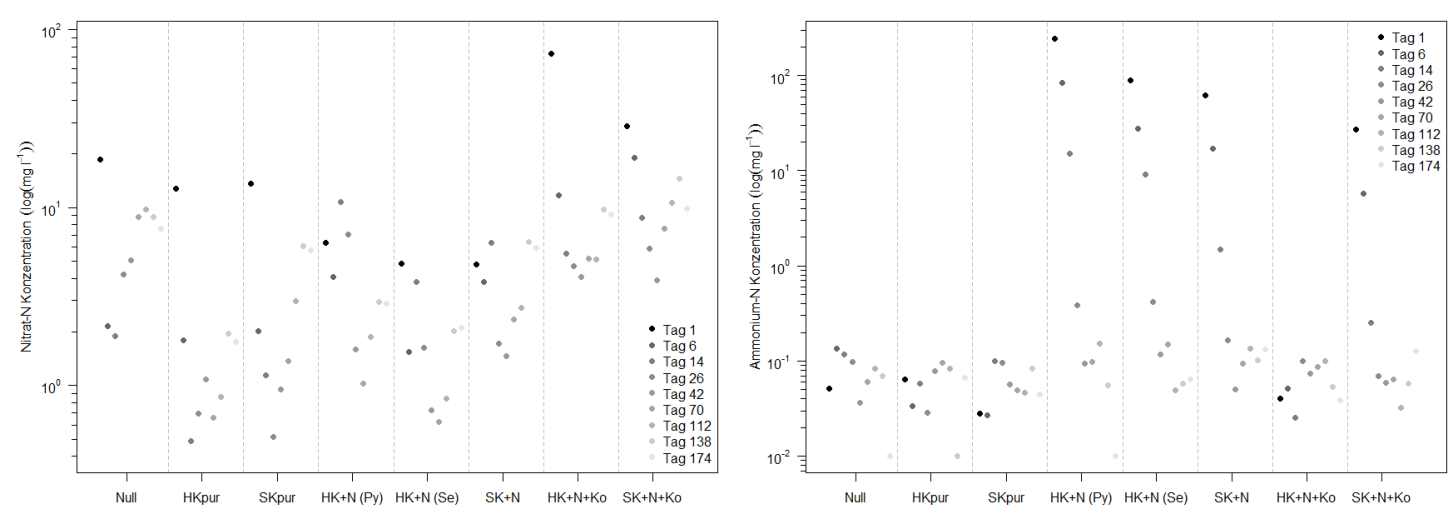

Abbildung 3.10: Logarithmische Darstellung der mittleren Nitrat-N- (links) und Ammonium-NKonzentration (rechts) im Extraktionsversuch, $n=2$.

Nach etwa 40 Tagen liegen die Nitratkonzentrationen in den jeweiligen Varianten auf einem stabilen Niveau bzw. steigen stetig an und zeigen einen Anstieg der Mineralisation. Dabei weist die Nullvariante zusammen mit den kompostbehandelten Kohlevarianten $(\mathrm{HK}+\mathrm{N}+\mathrm{Ko}$ und $\mathrm{SK}+\mathrm{N}+\mathrm{Ko}$ ) die höchsten Werte auf. Die übrigen Substrate zeigen ab dem 42. Tag weniger Nitrataustrag als die kohlefreie Nullvariante auf. Die Ergebnisse lassen zunächst vermuten, dass die Nullvariante die organische Masse stärker abbaut als die puren und stickstoffnachbehandelten $(+\mathrm{N})$ Kohlevarianten. Die Ergebnisse des Feld- und Topfversuches (Sickerwasserausträge) zeigen jedoch ein sehr hohes Potenzial der Nitratadsorption durch das Kohlesubstrat für die Kohlevarianten. Die Mineralisierung in den Kohlevarianten muss also nicht unbedingt niedriger als in der Nullvariante sein. Die Endprodukte der Mineralisierung werden von den Kohlen stark adsorbiert und werden nicht mit dem Sickerwasser transportiert.

Im Vergleich zur Variante HKpur weist die Variante SKpur einen deutlich höheren Anstieg der Nitratausträge auf. Geringere Nitratsorptionsraten und/oder höhere Mineralisierungsraten können dafür verantwortlich sein.

Mit Ausnahme der Variante HK $+\mathrm{N}+$ Ko zeigen die stickstoff- $(+\mathrm{N})$ und kompost- $(+\mathrm{N}+\mathrm{Ko})$ behandelten Kohlevarianten in den ersten 40 Tagen die höchsten Ammoniumausträge (siehe Abb. 3.10 (rechts)). Nach diesem Zeitraum verweilen die Ammoniumkonzentrationen auf einem geringen Niveau und weisen nur geringe Unterschiede zwischen den Varianten auf. Die Ergebnisse deuten auf hohe Nitrifikationsraten in den Substraten, eine hohe mikrobielle Aufnahme oder eine hohe Ammoniumbindefähigkeit an den negativen Austauscherplätzen der Kohlensubstrate. 


\subsubsection{Begleitvegetation auf den Versuchsparzellen}

\subsubsection{Deckungsgrad der Begleitvegetation}

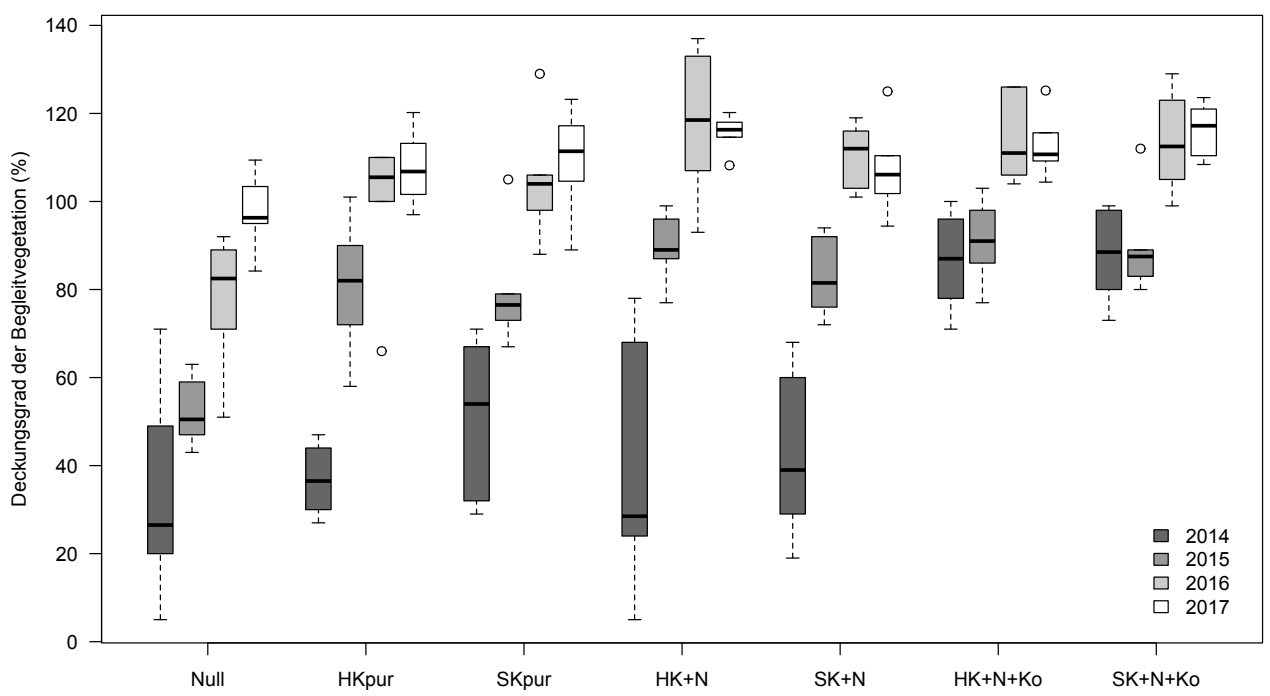

Abbildung 3.11: Deckungsgrad der Begleitvegetation auf den Versuchsparzellen $2014-2017, \mathrm{n}=6$.
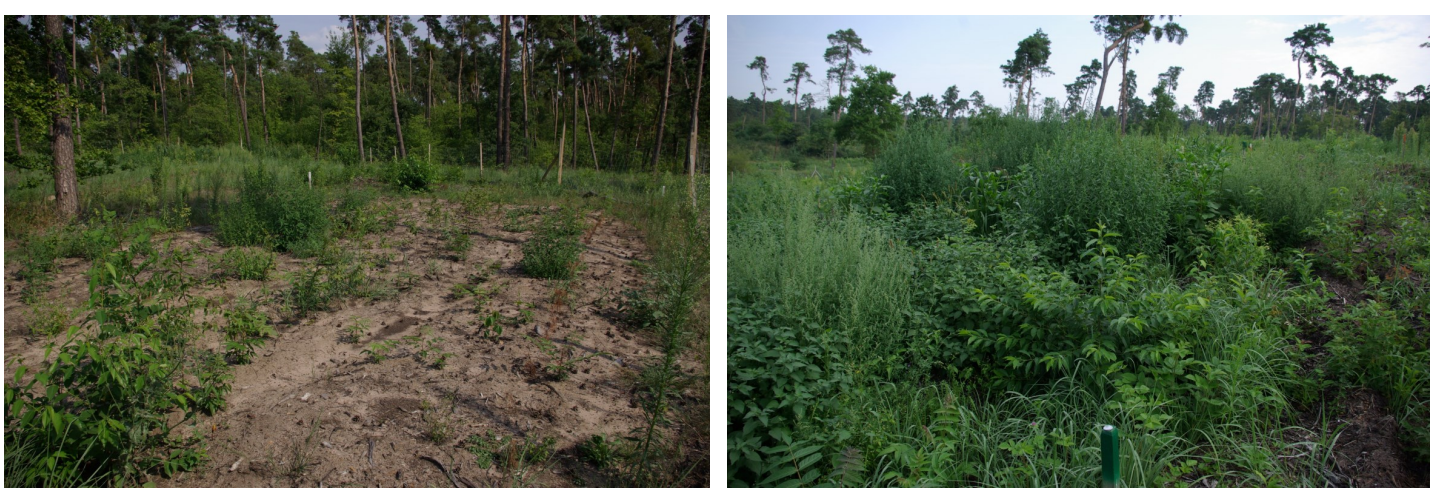

Abbildung 3.12: Nullparzelle (links) und Parzelle der Variante HK+N+Ko (rechts), Sommer 2014.

Der Gesamt-Deckungsgrad der Begleitvegetation ist im Jahr 2014 auf den Parzellen der kompostnachbehandelten Kohlevarianten $\mathrm{HK}+\mathrm{N}+\mathrm{Ko}$ und $\mathrm{SK}+\mathrm{N}+\mathrm{Ko}$ deutlich höher als auf den Parzellen der übrigen Varianten (siehe Abb. 3.11). In den Jahren 2015, 2016 und 2017 steigen die Deckungsgrade in allen Varianten deutlich an, wobei die Nullvariante in allen Jahren den geringsten Deckungsgrad der Begleitvegetation aufweist. Deckungsgrade über $100 \%$ können durch eine gegenseitige Überdeckung der Pflanzen zustandekommen. Einen guten Eindruck geben die Bilder einer typischen Parzelle der Nullvariante (Abb. 3.12, links) und einer typischen Parzelle der Variante HK+N+Ko (Abb. 3.12, rechts). 


\subsubsection{Arten der Begleitvegetation}

In der Begleitvegetation auf den Parzellen gibt es deutliche Veränderungen in der Artzusammensetzung zwischen den Jahren 2014 bis 2017 (siehe Tab. 3.9 auf nachfolgender Seite). Während 2014 auf den Flächen der mit stickstoff- $(+\mathrm{N})$ und kompost- $(+\mathrm{N}+\mathrm{Ko})$ nachbehandelten Kohlevarianten vor allem nitrophile Arten wie Schwarzer Nachtschatten (Solanum nigrum L.) und Weißer Gänsefuß (Chenopodium album L.) vorkamen, waren diese Arten 2015 mit deutlich geringeren Deckungsgraden vertreten. Bei der Aufnahme 2016 konnte die Hühnerhirse (Echinochloa crus-galli (L.) P.BeAuv.) mit geringen Deckungsgradanteilen wieder aufgefunden werden. Die Grasvegetation bestehend aus Land-Reitgras (Calamagrostis epigejos (L.) Rотн), Roter Borstenhirse (Setaria pumila (Poir.) Roem. \& Schult.) und Rotem Straußgras (Agrostis capillaris L.) hat während der Jahre 2014 bis 2017 kontinuierlich zugenommen.

\subsubsection{Festlegung des Stickstoffs durch die Vegetation}

Die Höhe, der durch das PhytoCalc-Modell berechneten Biomasse und der entsprechenden Stickstoffbindung durch die Vegetation ist von Art, Deckungsgrad und Sprosslänge abhängig und baut sich bei allen Varianten außer den kompostbehandelten $(+\mathrm{N}+\mathrm{Ko})$ Kohlevarianten zwischen 2014 und 2016 kontinuierlich auf (siehe Tab. 3.10). Im Jahr 2017 zeigt sich mit Ausnahme der Nullvariante eine abnehmende Stickstoffbindung.

Tabelle 3.10: Mittlere Stickstofffixierung in der Vegetation von 2014 - $2017\left(\mathrm{~kg} \mathrm{ha}^{-1}\right)$, (Standardabweichung), $(\mathrm{n}=6)$.

\begin{tabular}{ccccc}
\hline Variante & 2014 & 2015 & 2016 & 2017 \\
\hline Null & $12,2(8)$ & $26,0(8,6)$ & $36,1(10,5)$ & $37,2(3,1)$ \\
HKpur & $15,9(4,3)$ & $39,6(8,7)$ & $50,4(10,9)$ & $40,2(2)$ \\
SKpur & $21,4(9,5)$ & $35,2(8,2)$ & $51,3(11,4)$ & $42,2(4,9)$ \\
HK+N & $21,4(18)$ & $41,4(7,9)$ & $63,1(17,5)$ & $44,8(1,7)$ \\
SK+N & $19,4(8,5)$ & $41,7(9,6)$ & $54,6(8,9)$ & $41,3(3,9)$ \\
HK+N+Ko & $52,1(10)$ & $45,1(3,8)$ & $57,0(11,2)$ & $42,3(4,6)$ \\
SK+N+Ko & $51,6(12,1)$ & $42,5(6,2)$ & $59,6(16,7)$ & $43,0(1,9)$ \\
\hline
\end{tabular}




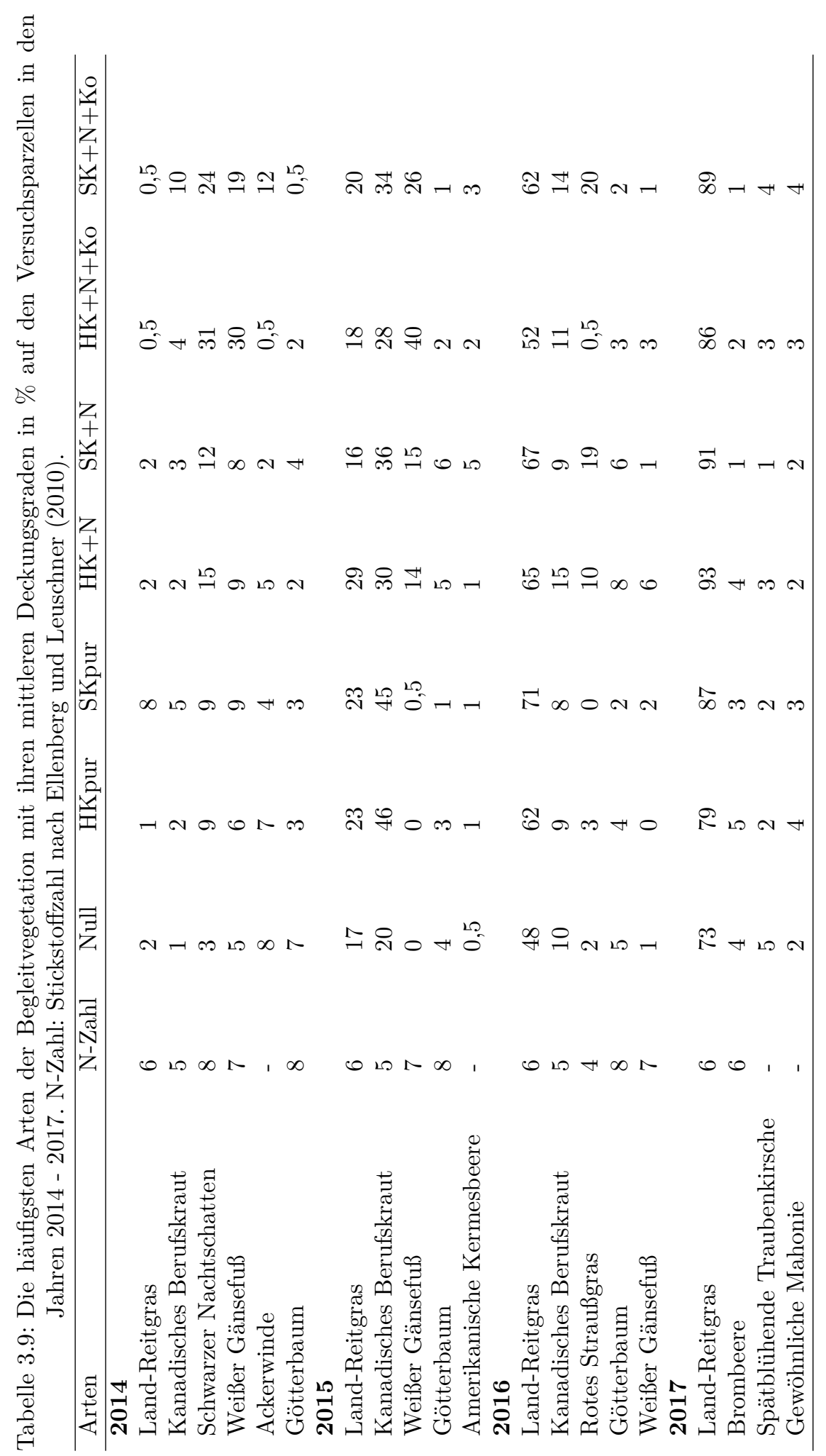




\subsubsection{Zusammenfassung Umweltfaktor Stickstoff}

Zusammenfassend lassen sich für den Umweltfaktor Stickstoff folgende Aussagen treffen:

Der Stickstoffvorrat wird durch die Kohleeinbringung je nach Variante zwischen 8 und $30 \%$ erhöht. Die Spelzenkohlevarianten haben einen höheren Stickstoffeintrag als die Holzkohlevarianten zur Folge.

Während der Anfangsphase (2014 und 2015) lassen sich sehr hohe Nitrat- und Ammoniumausträge über das Sickerwasser der stickstoff- $(+\mathrm{N})$ und kompost $(+\mathrm{N}+\mathrm{Ko})$ nachbehandelten Kohlevarianten beobachten. Dies lässt eine außergewöhnlich hohe Stickstoffverfügbarkeit während dieses Zeitraumes vermuten. Die Dynamik und die Artenzusammensetzung in der Begleitvegetation bekräftigt diese Vermutung und lässt wesentliche Wirkungen für die Vitalität der Hainbuchen und Kiefern erwarten. Die Stickstoffbindung über die Begleitvegetation und die Stickstoffaufnahme durch die Vegetation ist bei den Kohlevarianten stärker ausgeprägt als bei der Nullvariante. Indikatoren sind höhere Deckungsgrade und nitrophilere Arten mit höheren Stickstoffgehalten in den Geweben.

Die stickstoff- $(+\mathrm{N})$ und kompost $(+\mathrm{N}+\mathrm{Ko})$ nachbehandelten Kohlevarianten zeigen im Stickstoffaustrag mit dem Sickerwasser kein saisonales Reaktionsmuster und liegen in der zweiten Phase des Feldversuches (ab 2016) unter der Nullvariante. Trotz des höheren Stickstoffvorrates in der annuellen Begleitvegetation dieser Varianten wird der Abbau dieser organischen Biomasse (Stickstoffmineralisierung) während der Nichtvegetationszeit nicht von Stickstoffausträgen mit dem Sickerwasser begleitet. Dies ist ein weiteres Indiz für eine hohe Stickstoffimmobilisierung dieser Kohlevarianten ab dem Jahr 2016.

In der zweiten Phase des Feldversuches (ab Sommer 2016) liegen die Stickstoffkonzentrationen in der Bodenlösung der Nullvariante über denen der übrigen Varianten. Zusammen mit den Ergebnissen des Extraktionsversuches lässt sich mit zunehmender Versuchsdauer ein sehr hohes Stickstoffspeicherpotenzial der stickstoff- $(+\mathrm{N})$ und kompost $(+\mathrm{N}+\mathrm{Ko})$ nachbehandelten Kohlevarianten ableiten.

Die Variante HK+N zeigt während der Anfangsphase im Sickerwasseraustrag einen unerwartet hohen Ammoniumaustrag auf einigen Parzellen. Diese Beobachtung bestätigt sich im Extraktionsund Topfversuch bei denen zwei nach Herstellern getrennet Versuchsvarianten HK+N untersucht werden. Die Variante HK+N (Py) des Herstellers Pyreg weist einen etwa $50 \%$ höheren Stickstoffgehalt auf und zeigt höhere Nitrat- und Ammoniumausträge als die Variante HK+N (Se) des Herstellers Sonnenerde. Es gibt daher Hinweise auf eine unterschiedliche Nachbehandlung der identischen Kohlenausgangssubstrate.

Die stickstofffreien puren Kohlesubstrate (HKpur bzw. SKpur) weisen im Feldversuch Unterschiede im Stickstoffaustrag auf. Zu Beginn liegen die Nitratausträge der Spelzenkohlevariante SKpur noch über der Holzkohlevariante HKpur. Dies kann in einer höheren Sorptionsfähigkeit der Holzkohle oder in höheren Mineralisierungsraten der Spelzenkohlevariante begründet sein. Ab 2016 liegen beide Substrate im Nitrataustrag etwa auf einem Niveau.

Die Stickstoffausträge in Feld- und Topfversuch zeigen ähnliche Muster. Der Topfversuch liefert zusätzlich Hinweise auf eine Aufnahmepräferenz der beiden Baumarten hinsichtlich der Stickstoffform. Die Kiefer zeigt eine höhere Nitrat- und eine geringere Ammoniumaufnahme als die Hainbuche. 


\subsection{Anpassungsreaktionen der Bäume in Abhängigkeit von Stickstoff- und Trockenstress}

\subsection{1 Überlebenszahlen}

\subsubsection{1 Überlebenszahlen im Feldversuch}
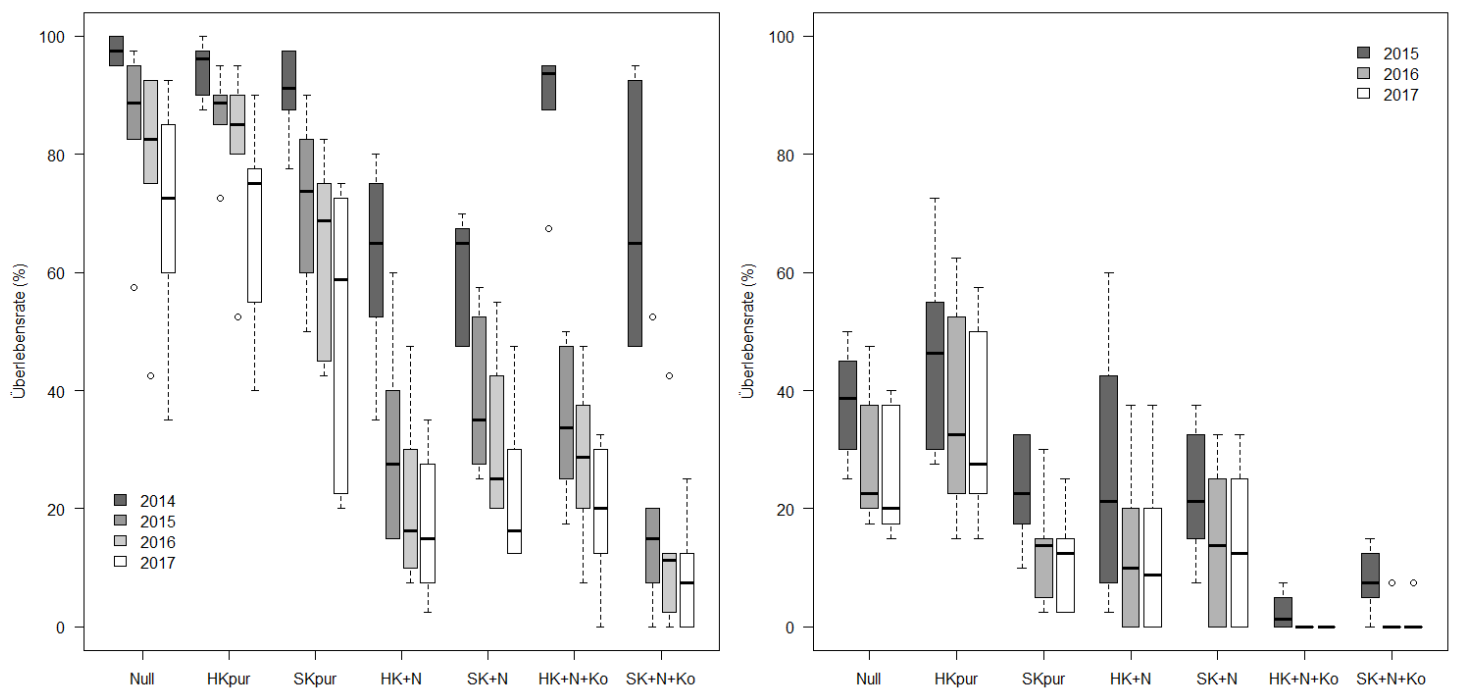

Abbildung 3.13: Mittlere Überlebensrate der Hainbuche in den Jahren 2014 - 2017 (links) und der 2015 gepflanzten Kiefer in den Jahren 2015 - 2017 (rechts) auf den Parzellen im Feldversuch, $\mathrm{n}=6$.

Die Anzahl der lebenden Hainbuchen nimmt während der Versuchsdauer kontinuierlich ab (siehe Tab. 3.11 und Abb. 3.13 (links)). Am deutlichsten ist der Rückgang bei den stickstoff- $(+\mathrm{N})$ und kompost- $(+\mathrm{N}+\mathrm{Ko})$ nachbehandelten Kohlevarianten. Die Varianten $\mathrm{HK}+\mathrm{N}$ und $\mathrm{SK}+\mathrm{N}$ zeigen schon nach einer Vegetationszeit im Herbst 2014 deutliche Verluste. Im Folgejahr (2015) haben die Varianten $\mathrm{HK}+\mathrm{N}, \mathrm{HK}+\mathrm{N}+\mathrm{Ko}, \mathrm{SK}+\mathrm{N}+\mathrm{Ko}$ die größten Verluste zu verzeichnen. Durch die Probenahme von Pflanzen im Zuge der Wurzeluntersuchungen 2015, 2016 und 2017 ist in allen Versuchsvarianten ein jährlicher Rückgang von etwa $4 \%$ verursacht worden.

Die Varianten Null und die puren Kohlevarianten (HKpur und SKpur) weisen im Jahr 2017 bei hoher Streuung die höchste Anzahl lebender Hainbuchen auf den Parzellen auf.

Die 2014 gepflanzten Kiefern zeigen bereits in der ersten Vegetationszeit sehr hohe Ausfallzahlen (siehe Tabelle 3.11, 7. Spalte). Besonders in den stickstoff- $(+\mathrm{N})$ und kompost- $(+\mathrm{N}+\mathrm{Ko})$ nachbehandelten Kohlevarianten ist ein vollständiges Absterben auf den meisten Parzellen zu beobachten. Während auf anderen Verjüngungsflächen im Forstamt Lampertheim, nach dem Standardverfahren Mulchen und Maschinenpflanzung, im Jahr 2014 nur etwa 30 \% Ausfälle zu verzeichnen sind, betragen die Verluste auf den Parzellen der Nullvariante fast 88 \%. Dies zeigt, dass durch die Versuchsvorbereitung möglicherweise weitere Faktoren hinzukommen, die das Überleben stark beeinflussen und in zukünftigen Anwendungen mit berücksichtigt werden sollten. Eventuell haben bodenvorbereitende Maßnahmen und Pflanztermin bzw. -verfahren einen 
großen Einfluss auf die Überlebensraten der Kiefer. Im Abschnitt 5.4 auf Seite 120 werden entsprechende Vorschläge aufgeführt.

Die Überlebenszahlen der 2015 neugepflanzten Kiefer sind bereits in der ersten Vegetationszeit deutlich zurückgegangen (siehe Abb. 3.13 (rechts)). Vor allem die Varianten $\mathrm{HK}+\mathrm{N}+\mathrm{Ko}$ und $\mathrm{SK}+\mathrm{N}+$ Ko zeigen einen Totalverlust der Kiefer. Die Variante HKpur zeigt wie schon im Vorjahr auch in den Folgejahren die höchsten Überlebensraten der Kiefer, gefolgt von der Nullvariante. Die Absterberaten waren zwischen den Inventurterminen 2016 und 2017 sehr gering, die Überlebensraten haben sich auf einem niedrigen Niveau stabilisiert.

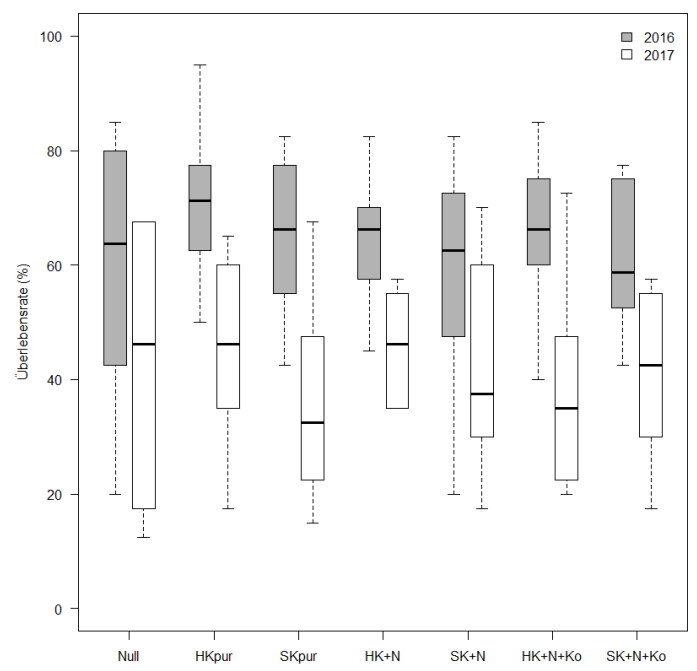

Abbildung 3.14: Mittlere Überlebensrate der 2016 gepflanzten Kiefer, 2016 und 2017, n=6.

Die 2016 gepflanzten Kiefern zeigen im Jahr 2016 im Vergleich zu den vorherigen Kiefernpflanzungen höhere und über alle Behandlungsvarianten ausgeglichene Überlebensraten zwischen 60 und $70 \%$ (siehe Abb. 3.14). Die Trockenperiode Anfang 2017 hatte jedoch große Auswirkungen auf die Überlebensraten der Kiefer. Es zeigen sich bei einer durchschnittlichen Überlebensrate von weniger als $50 \%$ leicht geringere Überlebensraten bei den Varianten $\mathrm{HK}+\mathrm{N}+\mathrm{Ko}$ und SKpur. 


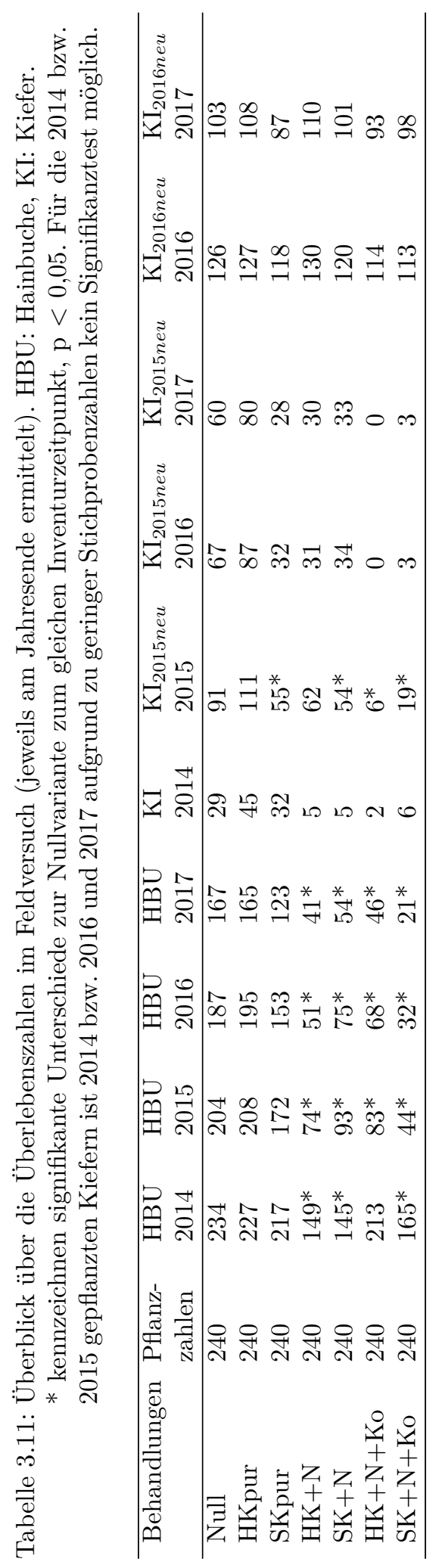




\subsubsection{2 Überlebensdauer im Topfversuch}
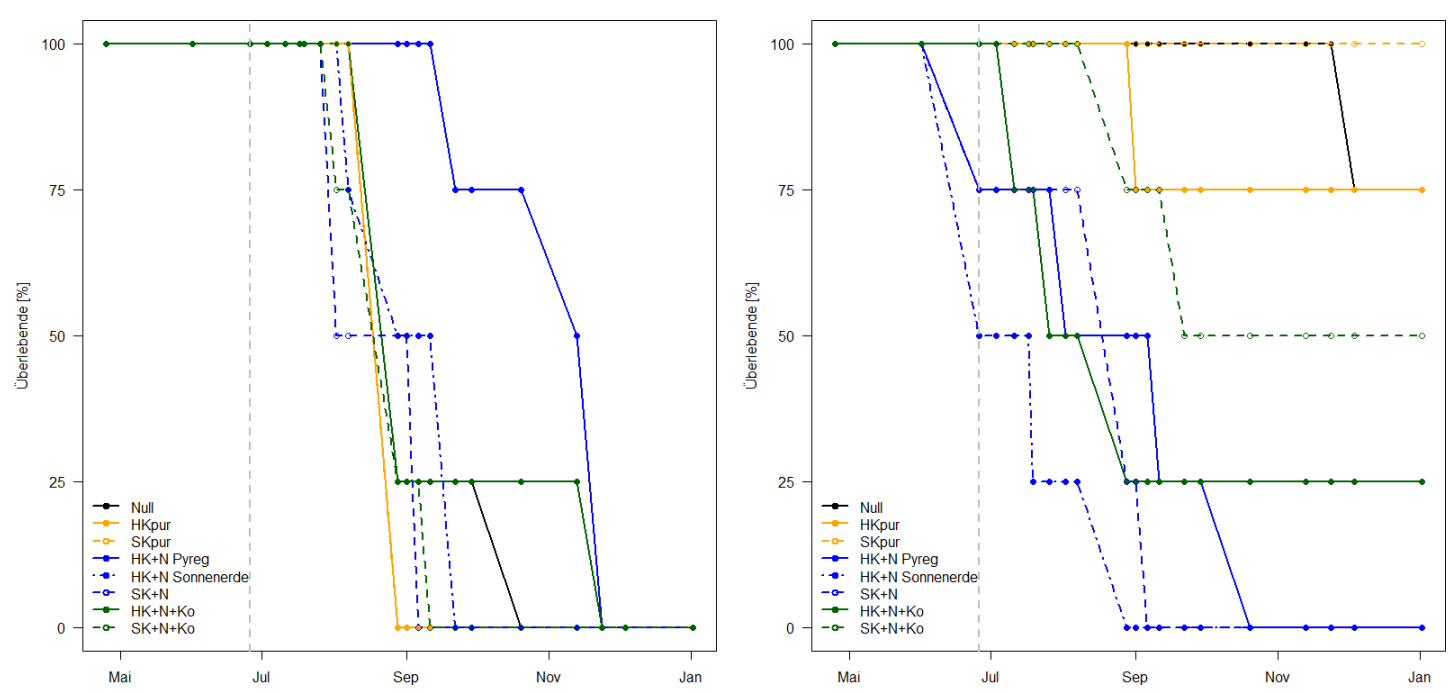

Abbildung 3.15: Mittlere Lebensdauer im Topfversuch im Jahr 2017. Hainbuche (links) und Kiefer (rechts). Die senkrechte gestrichelte graue Linie markiert das Ende der Bewässerungsperiode, $\mathrm{n}=4$.

Im Topfversuch zeigen die Hainbuchen erst 4 Wochen nach Ende der Bewässerungsphase erste Absterbeerscheinungen (siehe Abb. 3.15 (links)) bei den Varianten $\mathrm{SK}+\mathrm{N}, \mathrm{SK}+\mathrm{N}+\mathrm{K}$ und $\mathrm{HK}+\mathrm{N}$ (Sonnenerde). Die ersten kompletten Ausfälle aller Pflanzen zeigen die Varianten HKpur und SKpur, SK+N, SK+N+Ko. Die längste Überlebensdauer zeigen die Hainbuchen der Varianten $\mathrm{HK}+\mathrm{N}$ Pyreg und $\mathrm{HK}+\mathrm{N}+\mathrm{Ko}$.

Im Gegensatz zur Hainbuche zeigen die Kiefern beider HK+N Varianten (Pyreg und Sonnenerde) noch vor Ende der Bewässerungsperiode erste Absterbeerscheinungen (siehe Abb. 3.15 (rechts)) Die HK+N-Varianten und die SK+N-Variante weisen zuerst einen Komplettausfall aller Kiefern auf. Die Nullvariante und die Varianten SKpur und HKpur zeigen die längsten Überlebensdauern und höchsten Überlebensraten.

\subsubsection{Zusammenfassung der Überlebenszahlen}

In der Gesamtbetrachtung der Mortalität lässt sich feststellen, dass in der ersten Versuchsphase nach Kohleeinarbeitung in den Jahren 2014 und 2015 deutlich mehr Mortalitätsereignisse zu verzeichnen sind als in den nachfolgenden Jahren. Zwei Jahre nach Bodenbearbeitung und Kohleeinarbeitung zeigen die Pflanzen deutlich höhere Überlebensraten.

Die Kiefer weist deutlich höhere Mortalitätsraten als die Hainbuche auf und reagiert damit sensitiver auf die Anwuchsbedingungen. Beide Baumarten zeigen ähnliche Muster der Mortalität in Abhängigkeit von der Versuchsvariante. Die Parzellen der stickstoff- $(+\mathrm{N})$ und kompost$(+\mathrm{N}+\mathrm{Ko})$ nachbehandelten Kohlevarianten zeigen in der ersten Versuchsphase $(2014 / 2015)$ die meisten Mortalitätsereignisse. Die Hainbuchen der kompost- $(+\mathrm{N}+\mathrm{Ko})$ nachbehandelten Kohlevarianten mit einem Jahr Verzögerung. Somit weisen die Varianten Null und die puren Kohlevarianten (HKpur und SKpur), unter den Versuchsbedingungen, den besten Anwuchs auf. 


\subsubsection{Blatt-/Nadelzustand und Blattmorphologie}

\subsubsection{Blattverlust der Hainbuche}

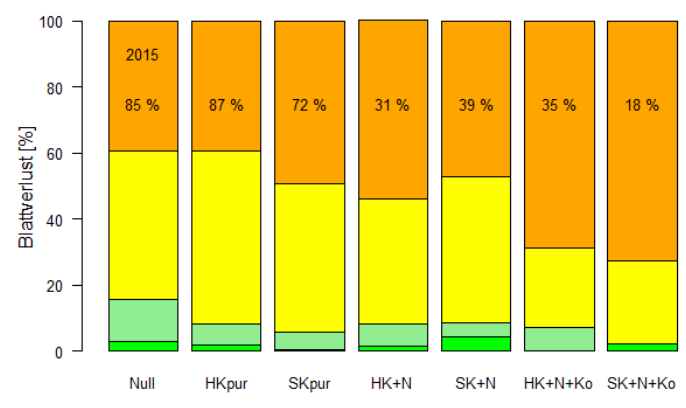

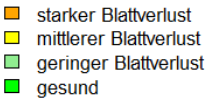

$\square$ gesund
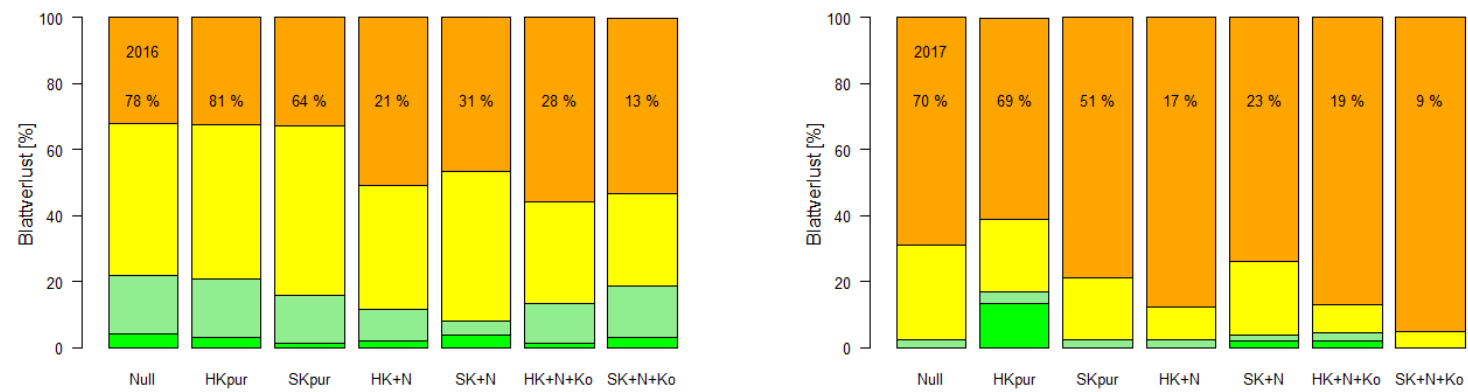

Abbildung 3.16: Blattverlust der Hainbuche in den Jahren 2015 - 2017. Durchschnittliche Überlebensrate (\%) der Versuchsvariante im jeweiligen Jahr in der Säule aufgetragen

Im Jahr 2015 war der Anteil der als gesund angesprochenen Pflanzen in den Varianten Null und $\mathrm{SK}+\mathrm{N}$ am höchsten (siehe Abb. 3.16). Den höchsten Anteil von Hainbuchen mit starkem Blattverlust weisen die Varianten $\mathrm{HK}+\mathrm{N}+\mathrm{Ko}$ und $\mathrm{SK}+\mathrm{N}+\mathrm{Ko}$ auf, den geringsten Anteil die Varianten Null und HKpur. Damit geht der Anteil starken Blattverlustes im Jahr 2015 mit den Überlebensraten im Folgejahr 2016 einher und kann als Frühindikator einer starken Mortalität angesehen werden.

Im Jahr 2016 konnte bei ausgeglichener Witterung ein insgesamt besserer Belaubungszustand der Hainbuche in allen Versuchsvarianten beobachtet werden. Wieder zeigten die Varianten Null und HKpur zusammen mit der Variante SKpur die geringsten Anteile und bei den Varianten $\mathrm{HK}+\mathrm{N}+\mathrm{Ko}$ und $\mathrm{SK}+\mathrm{N}+\mathrm{Ko}$ die höchsten Anteile starken Blattverlustes. Die stickstoff- $(+\mathrm{N})$ und kompost- $(+\mathrm{N}+\mathrm{Ko})$ nachbehandelten Varianten zeigten insgesamt einen schlechteren Belaubungszustand als die übrigen Varianten.

Der Belaubungszustand der Hainbuche ist im Jahr 2017 deutlich schlechter als in den Vorjahren. So lag der durchschnittliche Anteil starken Blattverlustes bei 79 \% (53\% im Jahr 2015 bzw. 43 \% im Jahr 2016). Der fehlende Blattneuaustrieb im Jahr 2017 hat möglicherweise Anteil an der schlechteren Bewertung der Belaubung. Auch die außergewöhnlich trockene Nichtvegetationszeit 2016/2017 und der trockene April 2017 könnten die Ursache des schlechten Belaubungszustand der Hainbuchen sein. Die Hainbuchen der Varianten Null und HKpur zeigen wieder die geringsten Anteile starken Blattverlustes. Die Variante HKpur weist zudem mit Abstand den höchsten Anteil gesunder Hainbuchen auf. 
Im Jahr 2015 und 2016 konnte bei einzelnen Hainbuchen nach dem Abwurf großer Teile der Blattmasse während der Trockenperioden ein Neuaustrieb im August/September vor allem der stark geschädigten Hainbuchen beobachtet werden (siehe Abb. 3.17). Eine Abhängigkeit zur Versuchsvariante konnte allerdings nicht beobachtet werden.

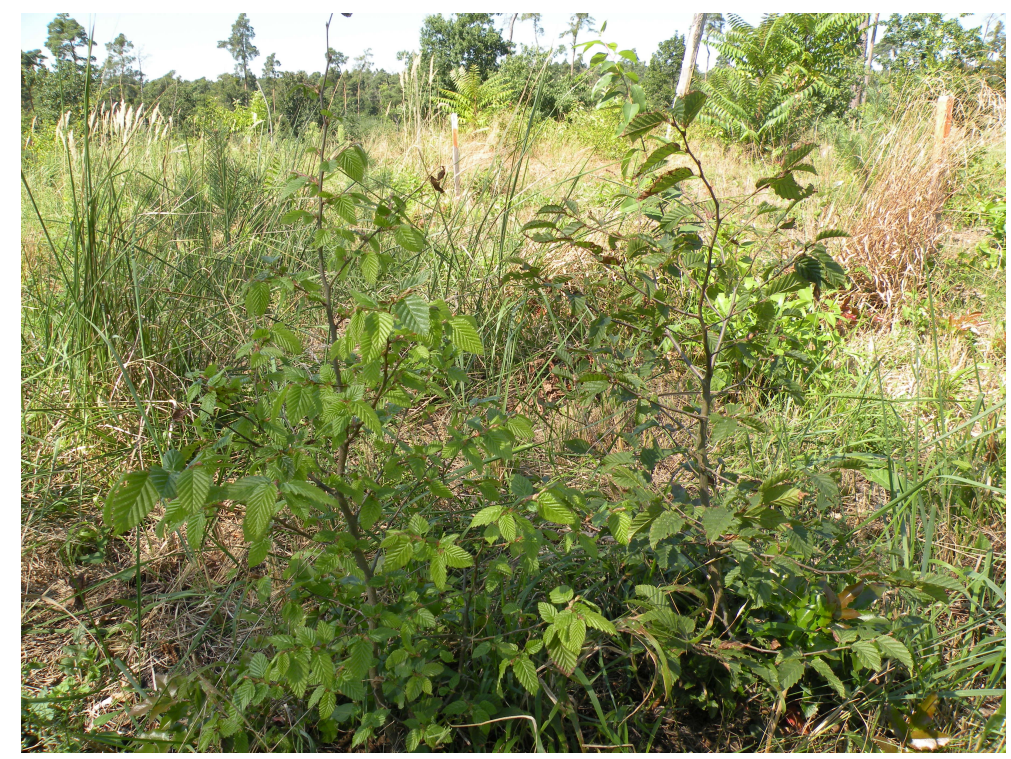

Abbildung 3.17: Neuer Blattaustrieb einer Hainbuche (linke Pflanze) im August 2016. Rechte Pflanze ohne neuen Blattaustrieb mit nekrotisierten Blatträndern.

\subsubsection{Nadelverfärbung der Kiefer}
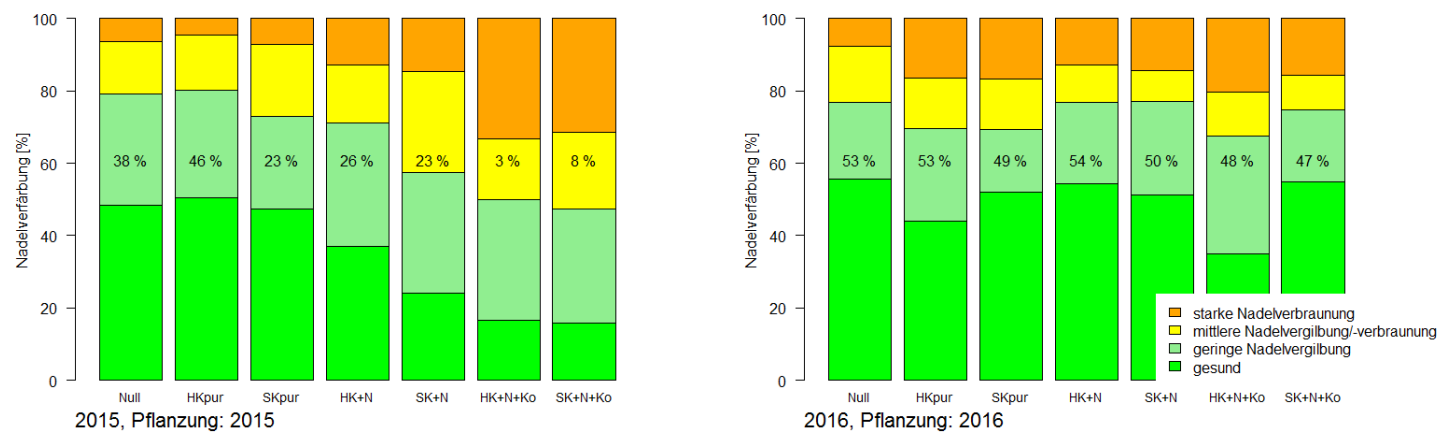

Abbildung 3.18: Nadelverfärbung der 2015 gepflanzten Kiefer im Jahr 2015 (links) und der 2016 gepflanzten Kiefer im Jahr 2016 (rechts). Durchschnittliche Überlebensrate (\%) der Versuchsvariante im jeweiligen Jahr in der Säule aufgetragen

Die 2015 gepflanzten Kiefern reagieren im Jahr 2015 mit ihrem Benadelungszustand auf die Stickstoffverfügbarkeit (siehe Abb. 3.18 (links)). Die kompostnachbehandelten $(+\mathrm{N}+\mathrm{Ko})$ Kohlevarianten weisen die höchsten Anteile starker Nadelverbraunung und die geringsten Anteile 
gesunder Pflanzen auf.

Die im Jahr 2016 gepflanzten Kiefern zeigen im Jahr 2016 leichte Unterschiede im Benadelungszustand auf (siehe Abb. 3.18 (rechts)). Die Nullvariante weist mit $56 \%$ den höchsten Anteil gesunder Pflanzen und mit $8 \%$ den geringsten Anteil stark geschädigter Pflanzen auf. Dagegen zeigt die Variante $\mathrm{HK}+\mathrm{N}+\mathrm{Ko}$ den höchsten Anteil stark geschädigter Pflanzen $(20 \%)$ und den geringsten Anteil gesunder Pflanzen (35 \%). Im Gegensatz zur Überlebensrate weisen die 2016 gepflanzten Kiefern also Unterschiede im Zustand der Benadelung auf.

\subsubsection{Blattflächen und Blatttrockenmassen im Feldversuch}
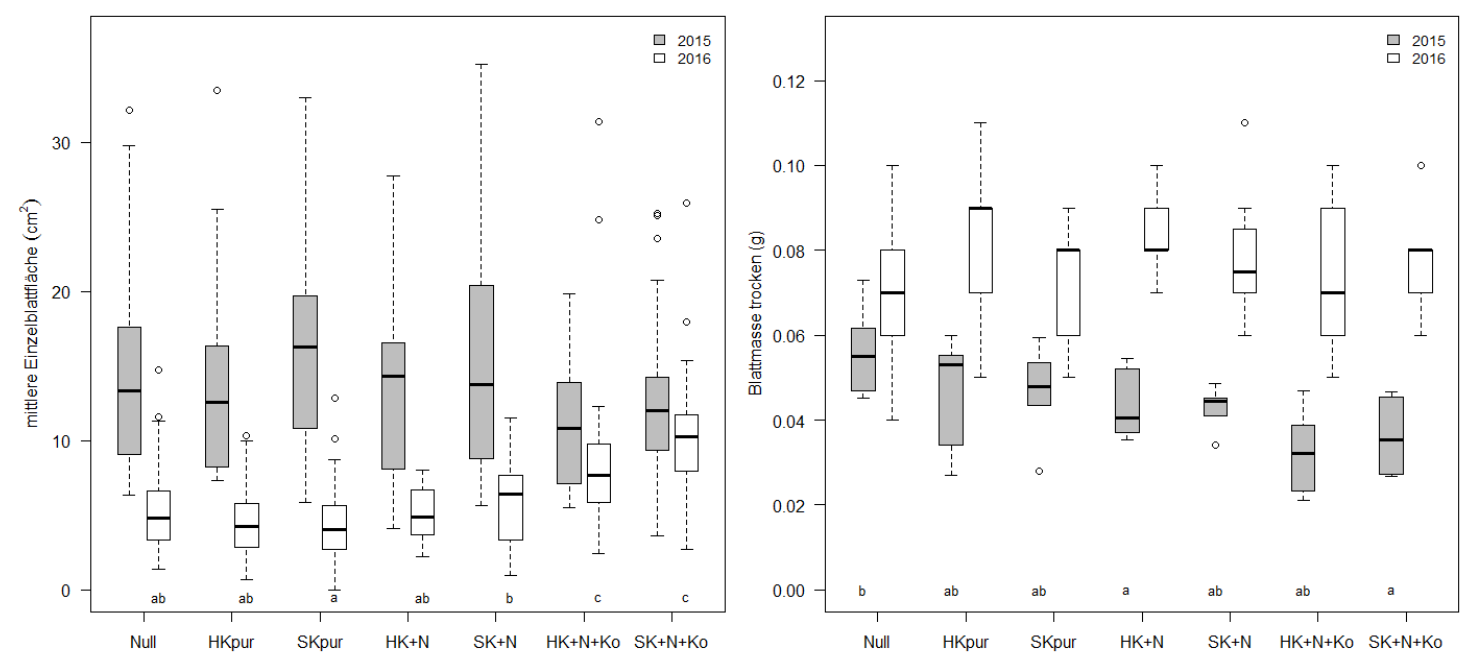

Abbildung 3.19: Einzelblattfläche (links) und -trockenmasse (rechts) der Hainbuche im Feldversuch 2015 und 2016. Unterschiedliche Buchstaben bezeichnen signifikante Unterschiede innerhalb eines Jahres (Signifikanzniveau $\mathrm{p}<0,05$ ).

Im Feldversuch weisen die Einzelblattflächen der Hainbuchenblätter im Jahr 2015 kaum Unterschiede zwischen den Behandlungen auf (siehe Abb. 3.19 (links)). Nach der trockenen Vegetationszeit im Jahr 2015 sind die beprobten Blätter im Jahr 2016 deutlich kleiner, am deutlichsten ist die Abnahme der Blattfläche in den Varianten Null, HKpur und SKpur zu beobachten. Die kompostbehandelten $(+\mathrm{N}+\mathrm{Ko})$ Kohlevarianten zeigen im Jahr 2016 signifikant höhere Blattflächen als die übrigen Varianten.

Es zeigen sich für die Hainbuchen der kompostnachbehandelten $(+\mathrm{N}+\mathrm{Ko})$ Kohlevarianten im Jahr 2015 signifikant geringere Einzelblatt-Trockenmassen als bei der Nullvariante (siehe Abb. 3.19 (rechts)). Im Jahr 2016 sind, trotz kleinerer Blätter, in allen Versuchsvarianten höhere Einzelblatt-Trockenmassen zu beobachten. 


\subsubsection{Zusammenfassung des Blatt-/Nadelzustandes und der Blattmorphologie}

Die Hainbuchen der Nullvariante zeigen (2015 und 2016 zusammen mit den puren Kohlevarianten) den geringsten Anteil an starken Blattverlusten. Die Hainbuchen der stickstoff- $(+\mathrm{N})$ und kompostbehandelten $(+\mathrm{N}+\mathrm{Ko})$ Kohlevarianten weisen höhere Anteile an starken Blattverlusten auf. Damit lassen sich die gleichen Muster wie in den Überlebensraten dieser Varianten beobachten.

Nach dem Abwurf großer Teile der Blattmasse während Trockenperioden zeigen stärker geschädigte Hainbuchen einen Neuaustrieb im August bzw. September der Jahre 2015 und 2016.

Auch die Kiefern der stickstoff- $(+\mathrm{N})$ und kompostbehandelten $(+\mathrm{N}+\mathrm{Ko})$ Kohlevarianten zeigen 2015 die höchsten Anteile an starker Nadelverbraunung und deutlich geringere Anteile an Bäumen ohne Nadelschädigungen.

Die Hainbuchen reagieren mit ihrem Blattzustand langfristiger als die Kiefer auf den ungünstigen Stickstoffstatus des Bodens. Die Hainbuchen der stickstoff- $(+\mathrm{N})$ und kompostbehandelten $(+\mathrm{N}+\mathrm{Ko})$ Kohlevarianten zeigen auch im Jahr 2017 noch höhere Blattverluste als die Hainbuchen Nullvariante und der puren Holzkohlevariante. Die Kiefern weisen dagegen schon im Jahr 2016 deutlich geringere Variantenunterschiede in der Nadelverfärbung auf.

Die Hainbuchen der kompostnachbehandelten $(+\mathrm{N}+\mathrm{Ko})$ Kohlevarianten zeigten im Jahr 2015 deutlich geringere Einzelblatt-Trockenmassen als die Hainbuchen der Null- und puren Kohlevarianten.

Nach einer außergewöhnlich trockenen Vegetationsperiode werden im Folgejahr durch die Hainbuche kleinere Blätter mit höherer Trockenmasse ausgebildet. Dieser Effekt der morphologischen Anpassung ist bei den Hainbuchen der kompostnachbehandelten $(+\mathrm{N}+\mathrm{Ko})$ Kohlevarianten weniger stark ausgeprägt. 


\subsubsection{Spross- und Wurzelmorphologie}

\subsubsection{Wurzelhalsdurchmesser und Jahrestrieblänge}
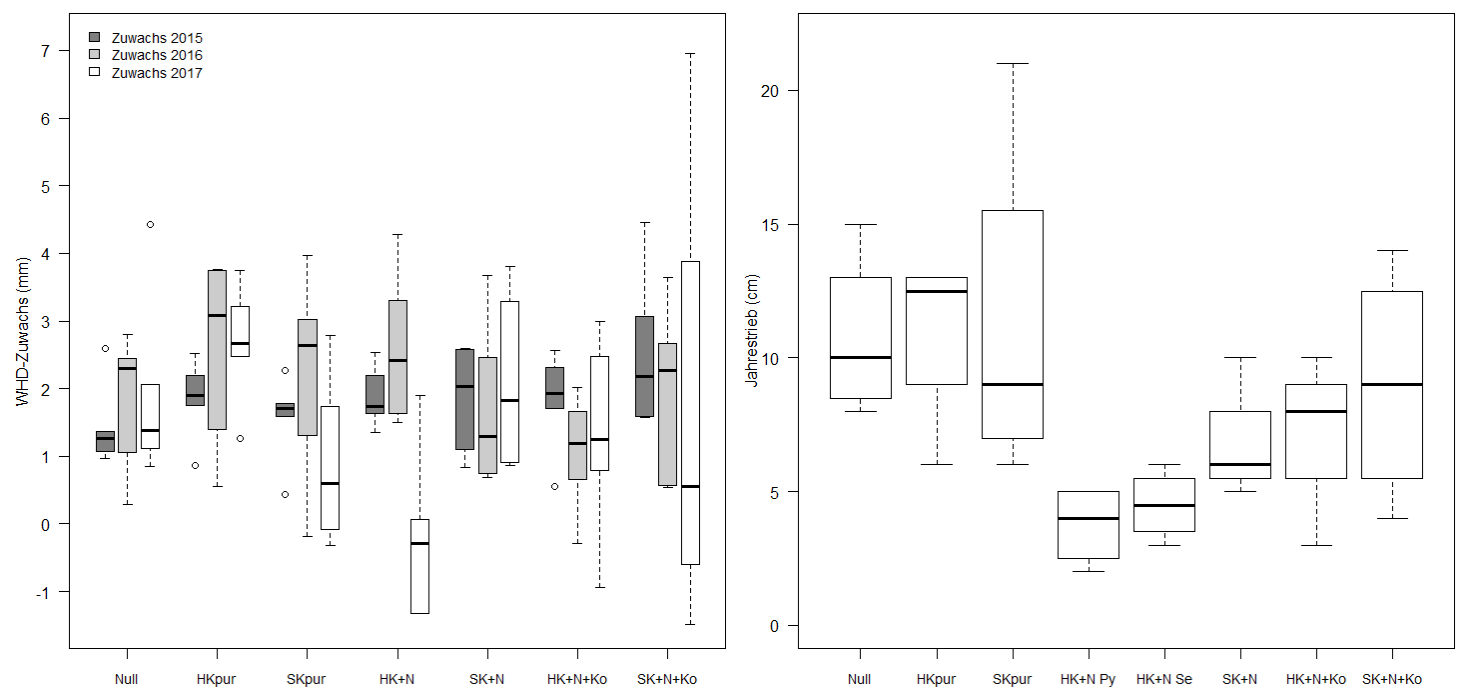

Abbildung 3.20: Zuwachs des Wurzelhalsdurchmessers (WHD) der Hainbuche in den Jahren 2015 bis 2017 (links) und der Jahrestrieblänge Kiefer im Topfversuch (rechts).

Der durchschnittliche Wurzelhalsdurchmesser von Hainbuche steigt mit der Versuchsdauer kontinuerlich an. Bei der Hainbuche zeigt der Zuwachs des Wurzelhalsdurchmessers im Jahr 2016 deutlich höhere Werte als in der trockenen Vegetationsperiode 2015 (siehe Abb. 3.20 (links)). Im Jahr 2015 zeigt die Nullvariante die niedrigsten Zuwächse im Wurzelhalsdurchmesser. Im Jahr 2017 weist der Wurzelhalsdurchmesserzuwachs der Hainbuche deutliche Unterschiede und eine höhere Streuung auf. Durch Absterbeerscheinungen vor allem stärkerer Hainbuchen in den Varianten $\mathrm{HK}+\mathrm{N}$ und $\mathrm{SK}+\mathrm{N}+$ Ko liegen die mittleren Wurzelhalsdurchmesser 2017 unter denen des Jahres 2016, deshalb erscheinen dort negative Zuwächse. Zu beachten sind die deutlich geringeren Stichprobenzahlen der stickstoff- $(+\mathrm{N})$ und kompost- $(+\mathrm{N}+\mathrm{Ko})$ nachbehandelten Kohlevarianten. Die Hainbuchen der Variante HKpur zeigt während der Versuchsdauer die größten Zuwächse im Wurzelhalsdurchmesser und weist dementsprechend 2017 die höchsten Wurzelhalsdurchmesser auf.

Im Topfversuch weisen die Jahrestriebe der Kiefern der Nullvariante und puren Kohlevarianten (HKpur bzw. SKpur) die größten Längen auf (siehe Abb. 3.20 (rechts)). Geringe Zuwächse sind bei den stickstoff $(+\mathrm{N})$ nachbehandelten Kohlevarianten zu verzeichnen. 


\subsubsection{Spross-Wurzel-Verhältnis der Trockenmassen}
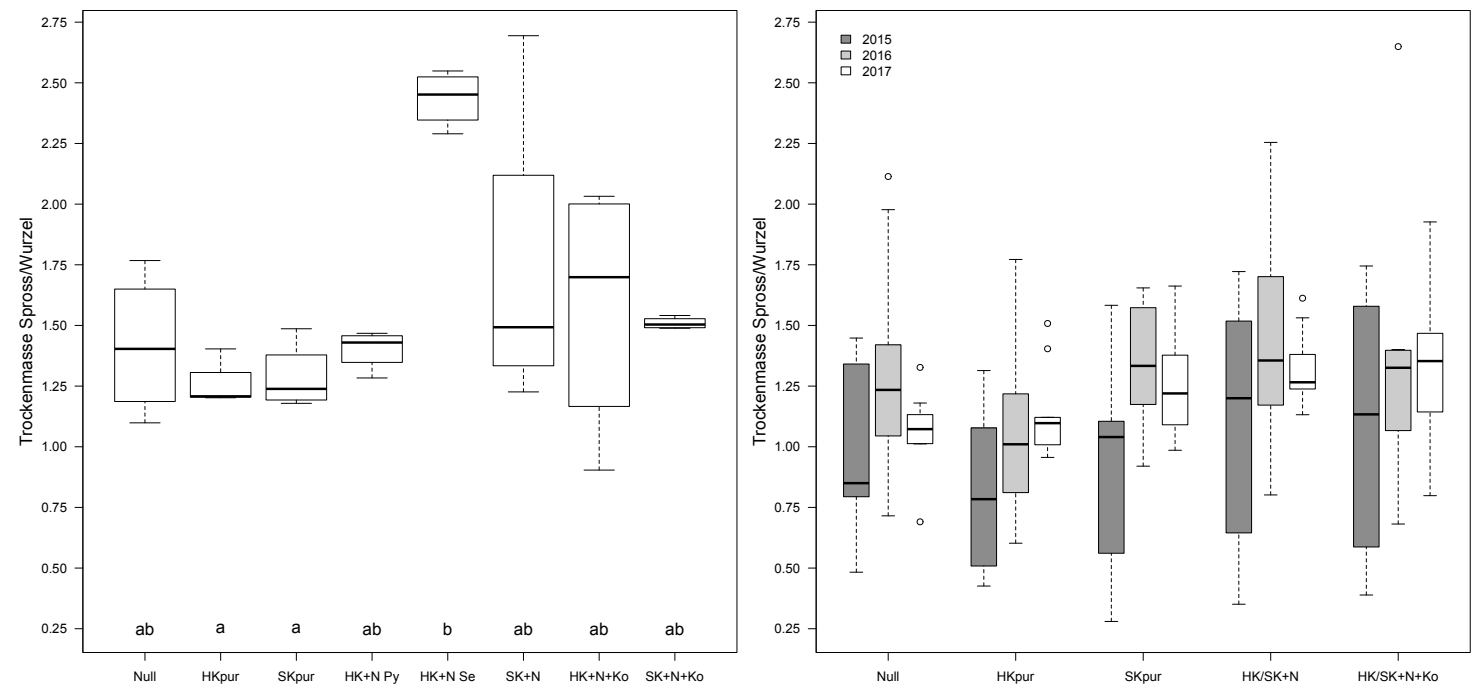

Abbildung 3.21: Spross-Wurzel-Verhältnis der Trockenmassen der Hainbuche im Topf- (links) und Feldversuch (rechts). Unterschiedliche Buchstaben bezeichnen signifikante Unterschiede innerhalb eines Jahres (Signifikanzniveau p $<0,05$ ), $\mathrm{n}=9$.

Beide Baumarten zeigen im Feldversuch mit fortschreitender Versuchsdauer ansteigende SprossWurzel-Verhältnisse.

Die Spross-Wurzel-Verhältnisse der Trockenmassen liegen bei der Hainbuche im Topfversuch höher als im Feldversuch (siehe Abb. 3.21). In beiden Versuchen wurden etwa $50 \mathrm{~cm}$ hohe Hainbuchen gepflanzt. Die Hainbuchen der puren Kohlevarianten (HKpur bzw. SKpur) weisen im Topfversuch signifikant geringere Werte des Spross-Wurzel-Verhältnisses auf als die Pflanzen der Variante HK+N (Se). Wie auch im Feldversuch zeigen die Hainbuchen der stickstoff- $(+\mathrm{N})$ und kompost- $(+\mathrm{N}+\mathrm{Ko})$ nachbehandelten Kohlevarianten tendenziell höhere Spross-WurzelVerhältnisse als bei der Nullvariante und puren Kohlevarianten. Die Wurzelsysteme sind im Verhältnis zum Spross weniger stark ausgeprägt. Es findet eine bevorzugte Allokation der Biomasse in oberirdische Pflanzenkompartimente statt. Damit zeigt die Hainbuche bereits nach kurzer Zeit eine deutliche Reaktion auf die hohe Stickstoffverfügbarkeit dieser Varianten. Dieses Reaktionsmuster dauert bei der Hainbuche auch während der folgenden Untersuchungen bis ins Jahr 2017 an, so weisen Hainbuchen der Variante HKpur in beiden Versuchen und in allen Versuchsjahren die geringsten Spross-Wurzel-Verhältnisse auf. 

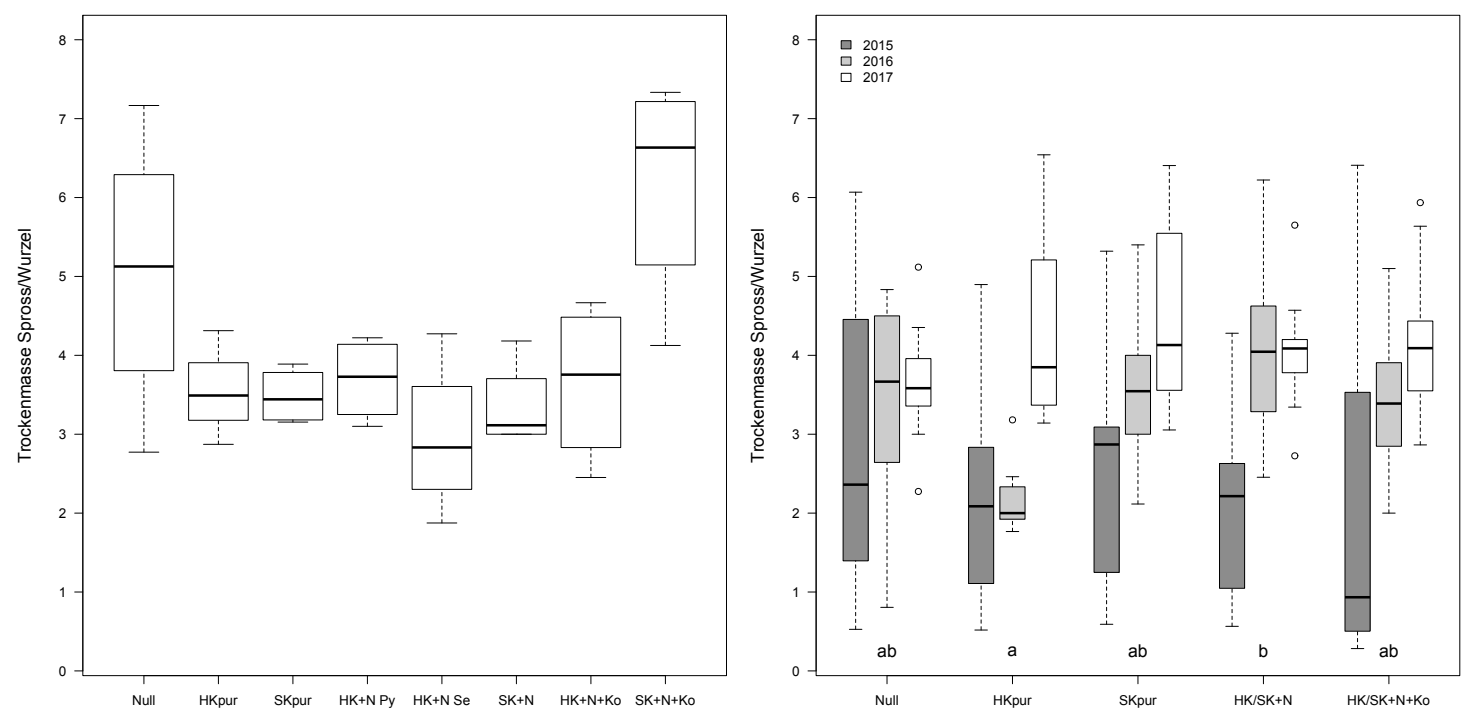

Abbildung 3.22: Spross-Wurzel-Verhältnis der Trockenmassen der Kiefer im Topf- (links) und Feldversuch (rechts). Unterschiedliche Buchstaben bezeichnen signifikante Unterschiede innerhalb eines Jahres (Signifikanzniveau $\mathrm{p}<0,05$ ), $\mathrm{n}=9$.

Die Spross-Wurzel-Verhältnisse der Kiefer zeigen im Vergleich zur Hainbuche deutlich höhere Werte (siehe Abb. 3.22). Auch hier liegen die Werte im Topfversuch durchweg höher als im Feldversuch. Im Feldversuch weisen die Kiefern der Variante HKpur 2016 signifikant geringere Spross-Wurzel-Verhältnisse als die Kiefern der stickstoffnachbehandelten $(+\mathrm{N})$ Kohlevarianten. Ein Jahresvergleich 2015 und 2016 ist jedoch nicht möglich, da im Jahr 2016 eine Neupflanzung mit einjährigen Kiefernpflanzen vorgenommen wurde. Bei der Pflanzung im Jahr 2015 wurden zweijährige Pflanzen verwendet.s

Im Gegensatz zur Hainbuche zeigt die Kiefer im Jahr 2017 kaum noch Unterschiede zwischen den Versuchsvarianten. Das Reaktionsmuster im Spross/Wurzel-Verhältnis wird bei der Kiefer somit weniger langfristig beibehalten. 


\subsubsection{Trockenmassen der Feinwurzeln}
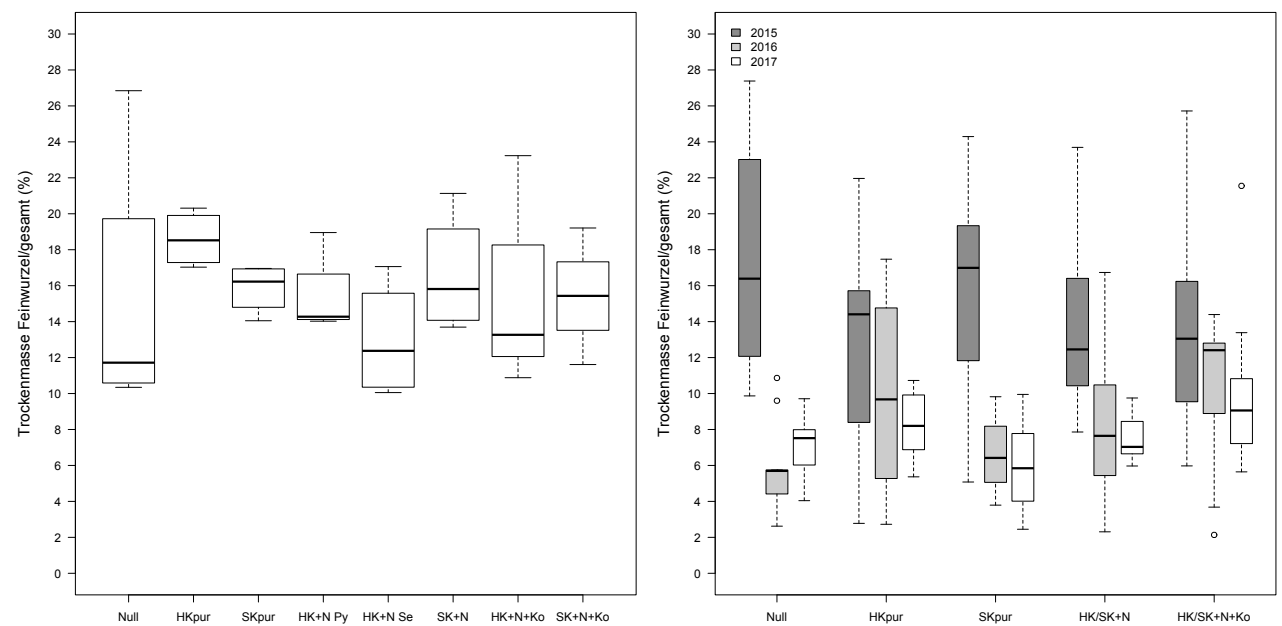

Abbildung 3.23: Anteil der Feinwurzeltrockenmasse an der Gesamttrockenmasse der Hainbuchen in $\%$ im Topf- (links) und Feldversuch (rechts), $\mathrm{n}=9$.

Der Anteil der Feinwurzeltrockenmasse an der Gesamttrockenmasse der Hainbuchen ist in Abbildung 3.23 dargestellt. Während der Feinwurzelanteil im Topfversuch und 2015 im Feldversuch zwischen 10 und $20 \%$ (Median) liegt, sind in den Jahren 2016 und 2017 geringere Feinwurzelanteile zwischen $6 \%$ und $12 \%$ (Median) zu beobachten. Ein Trend zu abnehmenden Feinwurzelanteilen an der Biomasse ist für den Untersuchungszeitraum für alle Kohlevarianten festzustellen. Die Nullvariante zeigt im Topfversuch mit $15 \%$ den geringsten Feinwurzelanteil, während sie im Jahr 2015 mit 18,1 \% (arithmetisches Mittel) den höchsten Wert aufweist.
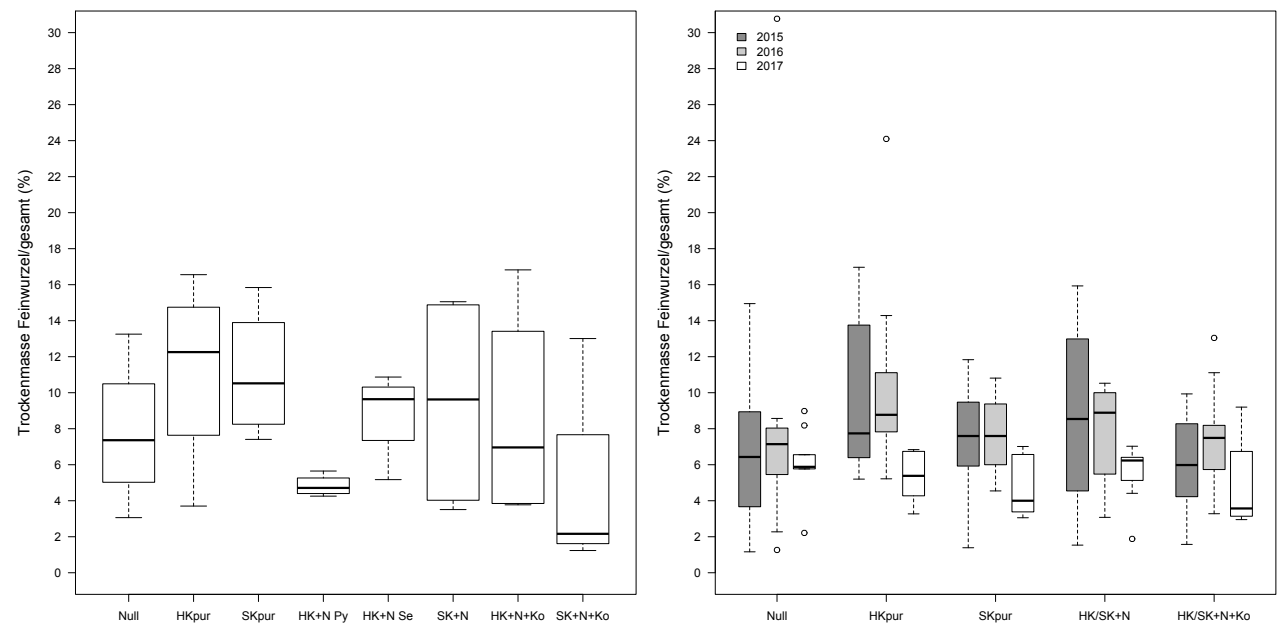

Abbildung 3.24: Anteil der Feinwurzeltrockenmasse an der Gesamttrockenmasse der Kiefer in \% im Topf- (links) und Feldversuch (rechts), $\mathrm{n}=9$. 
Im Baumartenvergleich zeigt die Kiefer einen deutlich geringeren Feinwurzelanteil an der Gesamttrockenmasse als die Hainbuche. Auffällig ist der geringere Feinwurzelanteil der Varianten $\mathrm{HK}+\mathrm{N}$ Py und SK $+\mathrm{N}+$ Ko im Topfversuch. Die Kiefernpflanzen der puren Kohlen (HKpur bzw. SKpur) weisen mit 12,6 \% bzw. 12,1 \% den höchsten Feinwurzelanteil im Topfversuch auf. Bei den Pflanzen der Variante HKpur lässt sich auch im Feldversuch in den Jahren 2015 und 2016 der höchste Feinwurzelanteil an der Gesamttrockenmasse beobachten (arithmetisches Mittel). Die Pflanzen der kompostbehandelten Kohlevarianten $(+\mathrm{N}+\mathrm{Ko})$ zeigen sowohl im Topfversuch (nur $\mathrm{SK}+\mathrm{N}+\mathrm{Ko}$ ) als auch im Feldversuch meist die geringsten Feinwurzelanteile.

\subsubsection{Anteile an mykorrhizierten und trockenen Feinwurzelspitzen}

Der Mykorrhizierungsgrad der Feinwurzeln der Hainbuche liegt nach drei Vegetationszeiten im Jahr 2016 im Median über $80 \%$ und weist signifikante Unterschiede auf (siehe Abb. 3.25 (links)). Die Nullvariante und puren Kohlevarianten (HKpur und SKpur) liegen etwa auf gleichem Niveau und zeigen die geringsten Anteile mykorrhizierter Wurzelspitzen. Die nachbehandelten Kohlevarianten mit Stickstoff $(+\mathrm{N})$ und Kompost $(+\mathrm{N}+\mathrm{Ko})$ zeigen höhere Mykorrhizierungsgrade der Feinwurzeln.

Die 2016 gepflanzte Kiefer weist nach nur einer Vegetationszeit ähnlich hohe Mykorrhizierungsgrade wie die Hainbuche auf. Die Kiefernfeinwurzeln der stickstoff- $(+\mathrm{N})$ und kompost- $(+\mathrm{N}+\mathrm{Ko})$ nachbehandelten Kohlevarianten weisen tendenziell geringere Werte als die der puren Kohlen auf. Damit kann im Baumartenvergleich für die stickstoff- $(+\mathrm{N})$ und kompost- $(+\mathrm{N}+\mathrm{Ko})$ nachbehandelten Kohlevarianten eine gegenläufige Reaktion in der Wurzelmykorrhizierung festgestellt werden.
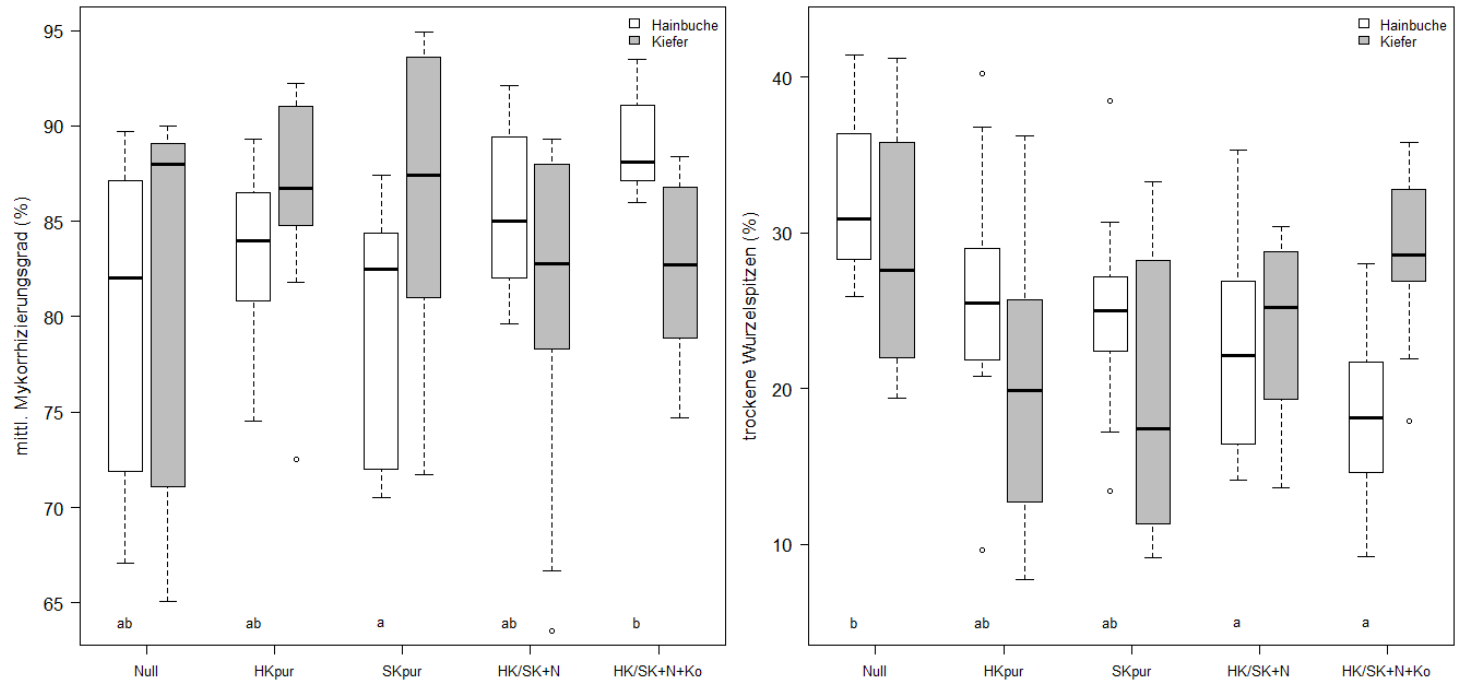

Abbildung 3.25: Links: Mykorrhizierungsgrad der Feinwurzeln von Hainbuche und Kiefer im Oktober 2016 in \%. Rechts: Anteil trockener Feinwurzelspitzen Hainbuche und Kiefer im Oktober 2016 in \%. Unterschiedliche Buchstaben bezeichnen signifikante Unterschiede innerhalb einer Baumart (Signifikanzniveau $\mathrm{p}<0,05$ ). 
Die untersuchten Hainbuchen der Nullvariante weisen im Jahr 2016 mit $32 \%$ einen signifikant höheren Anteil trockener Wurzelspitzen auf als die Hainbuchen der stickstoff- $(+\mathrm{N})$ und kompost$(+\mathrm{N}+\mathrm{Ko})$ nachbehandelten Kohlevarianten mit $23 \%$ bzw. $18 \%$ (siehe Abb. 3.25 (rechts)). Bei der Kiefer liegt der Anteil trockener Wurzelspitzen mit Ausnahme der stickstoff- $(+\mathrm{N})$ und kompost$(+\mathrm{N}+\mathrm{Ko})$ nachbehandelten Kohlevarianten niedriger als bei der Hainbuche der gleichen Variante. Die puren Kohlevarianten weisen weniger trockene Feinwurzelspitzen auf. Dieser Effekt ist jedoch nicht signifikant.

\subsubsection{Arten der Mykorrhiza}

Die auf den Feinwurzeln gefundenen und eindeutig morphologisch bestimmbaren Arten bzw. Gattungen waren:

- Genococcum geophilium

- Arten der Gattung Inocybe spec.

- Arten der Gattung Xerocomus spec.

- Arten der Gattung Tomentella spec. 


\subsubsection{Zusammenfassung der spross- und wurzelmorphologischen Reaktionen}

Als Reaktion auf die außergewöhnlich trockene Vegetationsperiode zeigen sich 2015 geringere Zuwächse im Wurzelhalsdurchmesser der Hainbuche als im Jahr 2016.

Die Kiefer zeigt den negativen Einfluss der stickstoff- $(+\mathrm{N})$ und kompost- $(+\mathrm{N}+\mathrm{Ko})$ nachbehandelten Kohlevarianten während der Initialphase des Versuches besonders deutlich. Die Kiefern der stickstoff $(+\mathrm{N})$ nachbehandelten Kohlevarianten haben im Topfversuch geringere Zuwächse im Jahrestrieb als die Kiefern der puren Kohlevarianten und der Nullvariante.

Die Hainbuchen der Variante HKpur zeigen besonders in den Jahren 2016 und 2017 die höchsten Zuwächse im Wurzelhalsdurchmesser.

Die Hainbuchen der stickstoff- $(+\mathrm{N})$ und kompost- $(+\mathrm{N}+\mathrm{Ko})$ nachbehandelten Kohlevarianten zeigen im gesamten Versuchszeitraum höhere Spross-Wurzel-Verhältnisse. Im Jahr 2017 ist bei der Kiefer keine Differenzierung im Spross-Wurzel-Verhältnis zu beobachten. Das Reaktionsmuster dauert somit weniger lang an, als bei der Hainbuche.

Im Jahr 2015 können geringere Spross-Wurzel-Verhältnisse und höhere Feinwurzeltrockenmassen an der Hainbuche beobachtet werden.

Die Hainbuchen der kompost- $(+\mathrm{N}+\mathrm{Ko})$ nachbehandelten Kohlevariante weisen tendenziell geringere Feinwurzeltrockenmassen auf.

Die Hainbuchen und Kiefern der Variante HKpur weisen geringe Spross-Wurzel-Verhältnisse und hohe Feinwurzeltrockenmassen auf.

Die Hainbuchen der kompostnachbehandelten $(+\mathrm{N}+\mathrm{Ko})$ Kohlevarianten weisen nach drei Vegetationsperioden höhere Mykorrhizierungsgrade an den Feinwurzeln auf. Die Reihenfolge absteigender Mykorrhizierung stellt sich folgendermaßen dar: $+\mathrm{N}+\mathrm{Ko}>+\mathrm{N}>$ pure Kohlen und Null. Die Anteile toter Wurzelspitzen zeigen einen reziproken Verlauf. Die Kiefern der stickstoff$(+\mathrm{N})$ und kompost- $(+\mathrm{N}+\mathrm{Ko})$ nachbehandelten Kohlevarianten zeigen dagegen nach einer Vegetationsperiode geringere Mykorrhizierungsgrade als die Kiefern der puren Kohlen- und der Nullvariante.

Die Hainbuchen der Nullvariante zeigen 2016 höhere Anteile trockener Wurzelspitzen als die Hainbuchen der stickstoff- $(+\mathrm{N})$ und kompost- $(+\mathrm{N}+\mathrm{Ko})$ nachbehandelten Kohlevarianten.

Damit ergeben sich Hinweise auf baumartenspezifische Reaktionsmuster gegenüber einer hohen Stickstoffverfügbarkeit. 


\subsubsection{Elementgehalte der Kompartimente Blatt/Nadel und Feinwurzel}

\subsubsection{Stickstoffgehalt und SPAD-Werte}
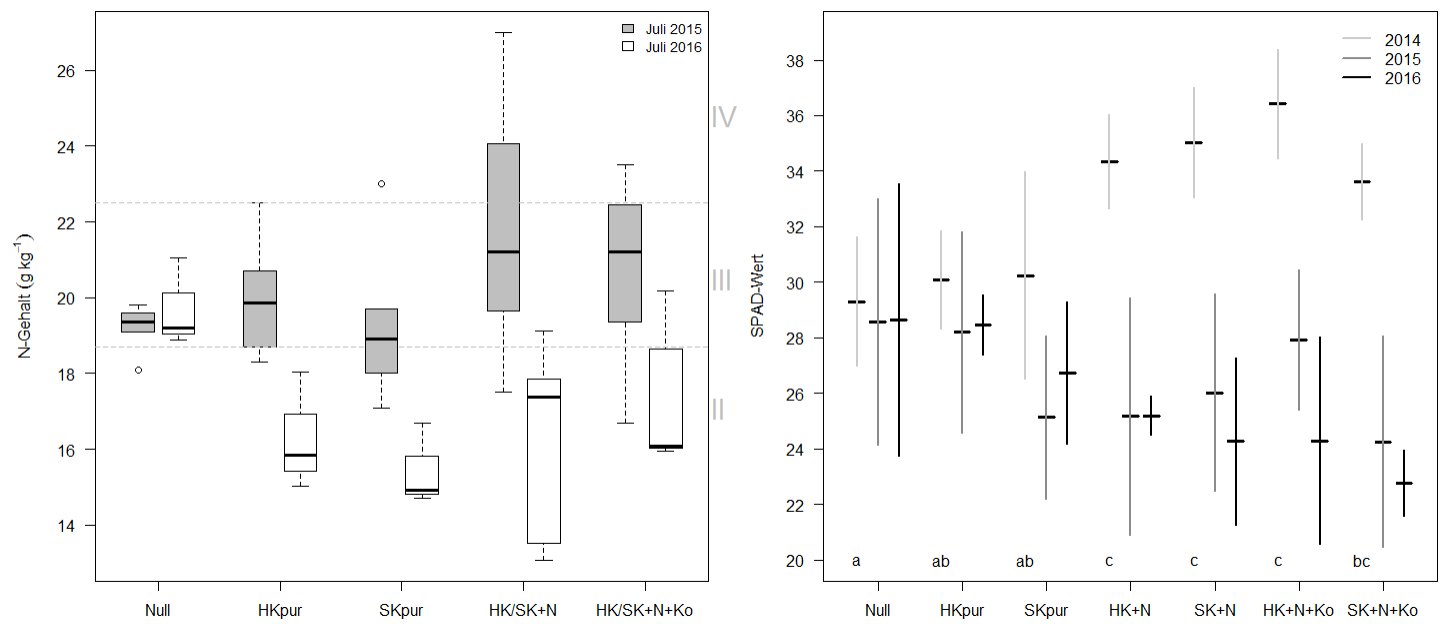

Abbildung 3.26: Links: Stickstoffgehalte der Hainbuchenblätter im Juli 2015 und 2016. Waagerechte gestrichelte Linien kennzeichnen die Grenzen des ausreichenden bis optimalen Versorgungszustandes nach Krauß und Heinsdorf (2005), n=6. Rechts: SPAD-Werte der Hainbuchenblätter in den Jahren 2014-2016. Mittelwerte mit Standardabweichungen des Mittelwertes. Unterschiedliche Buchstaben bezeichnen signifikante Unterschiede innerhalb eines Jahres (Signifikanzniveau $\mathrm{p}<0,05$, $\mathrm{n}=6)$.

Die Stickstoffgehalte der Hainbuchenblätter zeigen im Jahresvergleich 2015 und 2016 eine deutliche Abnahme in allen Kohlevarianten (siehe Abb. 3.26 (links)), während die Nullvariante weitgehend stabile Stickstoffgehalte aufweist. Im Jahr 2016 scheint die Stickstoffernährung der Hainbuchen der Kohlenvarianten im mangelhaften Versorgungszustand nach Krauß und Heinsdorf (2005) zu sein. Die Hainbuchen zeigen überraschend niedrige Werte im Stickstoffversorgungszustand.

Die SPAD-Werte als relatives Maß für den Chlorophyll- bzw. Stickstoffgehalt der Blätter zeigen im Jahr 2014 einen deutlichen Einfluss der Nachbehandlung der Kohlen (siehe Abb. 3.26 (rechts)). Die stickstoff- $(+\mathrm{N})$ und kompost- $(+\mathrm{N}+\mathrm{Ko})$ nachbehandelten Kohlevarianten unterscheiden sich signifikant von der Nullvariante und den puren Kohlevarianten (HKpur bzw. SKpur). Die Hainbuchen der puren Kohlevarianten (HKpur und SKpur) liegen 2014 im SPAD-Wert leicht über der Nullvariante. Im Folgejahr weisen beide Kohlevarianten niedrigere SPAD-Werte als die Hainbuche nder Nullvariante auf. Die Hainbuchen der Versuchsvarianten Null und pure Holzkohlen (HKpur und SKpur) weisen im Jahr 2015 ähnliche Stickstoffgehalte in den Blättern auf. Im Folgejahr liegen beide Kohlevarianten deutlich unter der Nullvariante. Die Stickstoffgehalte der Hainbuchenblätter folgen den SPAD-Werten mit einer zeitlichen Verzögerung. Die Hainbuchen der stickstoff- $(+\mathrm{N})$ und kompost- $(+\mathrm{N}+\mathrm{Ko})$ nachbehandelten Kohlevarianten zeigen 2015 und 2016 niedrigere SPAD-Werte als die Nullvariante. Im Blattstickstoffgehalt der Hainbuchen ist dieses Muster erst im Jahr 2016 zu beobachten.

Im Topfversuch weisen die Stickstoffgehalte der Hainbuchenblätter deutliche Unterschiede auf 
(siehe Abb. 3.27). Während die Hainbuchen der Nullvariante und der puren Kohlenvarianten (HKpur bzw. SKpur) im Mangelbereich der Stickstoffversorgung liegen, sind die stickstoff $(+\mathrm{N})$ und kompost $(+\mathrm{N}+\mathrm{Ko})$ nachbehandelten Kohlevarianten im ausreichenden bis optimalen Bereich. Die Variante HK $+\mathrm{N}$ Py liegt im Bereich der beginnenden Stickstoffüberversorgung nach Krauß und Heinsdorf (2005).

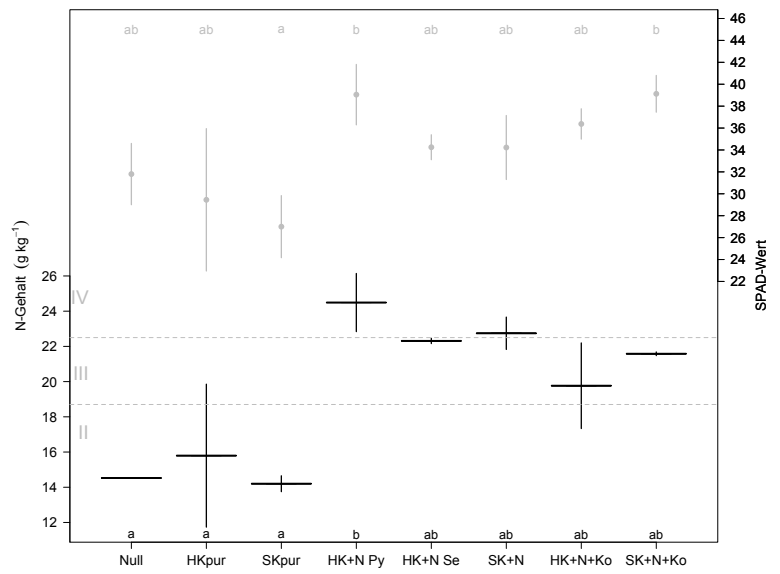

Abbildung 3.27: Stickstoffgehalte (schwarz, $n=4$ ) und SPAD-Werte (grau, $n=2$ ) der Hainbuchenblätter im Topfversuch am 13. Juli 2017. Mittelwerte mit Standardabweichungen des Mittelwertes. Waagerechte gestrichelte Linien kennzeichnen die Ober- und Untergrenze des ausreichenden bis optimalen Versorgungszustandes nach Krauß und Heinsdorf (2005). Unterschiedliche Buchstaben bezeichnen signifikante Unterschiede in einem Jahr (Signifikanzniveau $\mathrm{p}<0,05$ ).

Die am gleichen Termin ermittelten Stickstoff-Blattgehalte und SPAD-Werte zeigen ähnliche Muster. Die Gruppe der Nullvariante und der puren Kohlen (HKpur bzw. SKpur) zeigt deutlich niedrigere Stickstoffgehalte als die Gruppe der stickstoff- $(+\mathrm{N})$ und kompost- $(+\mathrm{N}+\mathrm{Ko})$ nachbehandelten Kohlevarianten. In beiden Untersuchungen zeigt die Variante HK+N Py die höchsten, die Variante SKpur die niedrigsten Werte. In beiden Untersuchungen unterscheiden sich diese Varianten signifikant.

Die Dynamik des SPAD-Wertes während einer Vegetationszeit zeigen die Abbildungen A.3 und A.4 im Anhang. Im Topfversuch bestehen an frisch ausgetriebenen Hainbuchenblättern kaum Unterschiede im SPAD-Wert, der sich im Laufe der Vegetationszeit kontinuierlich aufbaut und im Juli deutliche Unterschiede zwischen den Varianten zeigt. Die SPAD-Werte der puren Kohlevarianten (HKpur bzw. SKpur) liegen deutlich unter denen der anderen Kohlevarianten und der Nullvariante. Die Hainbuchen der stickstoff- $(+\mathrm{N})$ und kompost- $(+\mathrm{N}+\mathrm{Ko})$ nachbehandelten Kohlevarianten zeigen während der Dauer des Topfversuches den stärksten Anstieg im SPADWert. Die Hainbuchen der Varianten $\mathrm{HK}+\mathrm{N}(\mathrm{Py})$ und $\mathrm{SK}+\mathrm{N}+\mathrm{Ko}$ zeigen dabei die höchsten SPAD-Werte. Analog bestehen im Feldversuch zu Beginn der Vegetationszeit kaum Unterschiede zwischen den Versuchsvarianten. Mit fortschreitender Vegetationszeit steigen auch hier die SPAD-Werte langsam an.

Der Stickstoffgehalt in den Feinwurzeln der Hainbuche weist im Jahresvergleich 2015 und 2016 analog zu den Blattgehalten sinkende Werte auf (siehe Abb. 3.28 (links)). Auch hier fällt die 
Reduktion bei der Nullvariante am geringsten aus. Die Ergebnisse sprechen für eine sinkende Stickstoffverfügbarkeit zwischen beiden Jahren vor allem für die Hainbuchen der stickstoff- $(+\mathrm{N})$ und kompost- $(+\mathrm{N}+\mathrm{Ko})$ nachbehandelten Kohlevarianten.

Während im Jahr 2015 kaum Unterschiede zwischen den Varianten bestehen, differenziert sich die Stickstoffkonzentration im Jahr 2016 etwas stärker. Die Nullvariante weist zusammen mit Variante $\mathrm{SK}+\mathrm{N}$ die höchsten, die Variante SKpur die geringsten Werte auf.
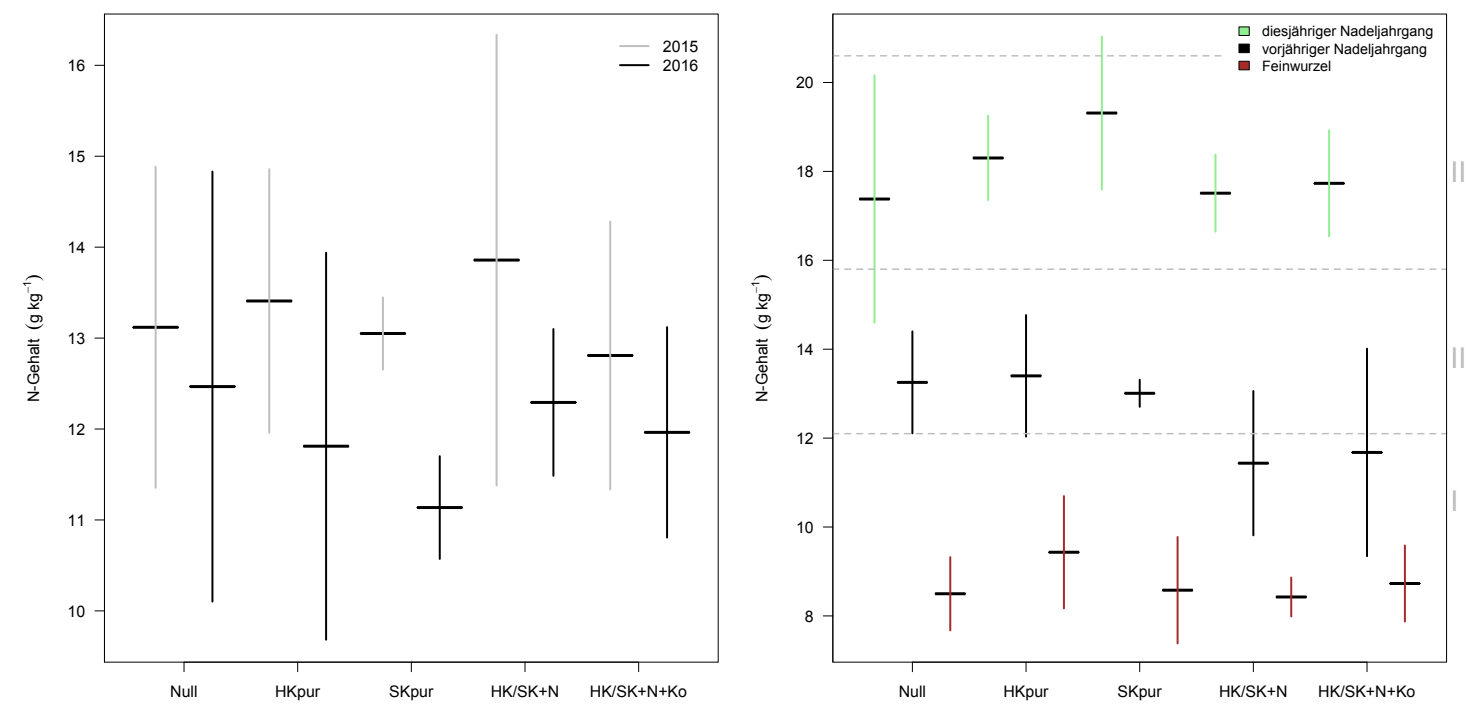

Abbildung 3.28: Links: Stickstoffgehalte der Hainbuchen-Feinwurzeln in den Jahren 2015 und 2016. Rechts: Stickstoffgehalte der dies- und letztjährigen Nadeljahrgänge und der Feinwurzeln der Kiefer im Jahr 2016. Mittelwerte mit Standardabweichungen des Mittelwertes. Waagerechte gestrichelte Linien kennzeichnen die Grenzen des Normalbereiches der Elementgehalte nach Krauß und Heinsdorf (2005).

Die 2016 gepflanzten Kiefern zeigen 2016 im diesjährigen Nadeljahrgang in allen Varianten eine ausreichend bis optimale Stickstoffversorgung nach Krauß und Heinsdorf (2005) (siehe Abb. 3.28 (rechts)). Die Kiefern der Variante SKpur liegen im Bereich der beginnenden Überernährung. Die Stickstoffgehalte der Kiefern der stickstoff- $(+\mathrm{N})$ und kompost- $(+\mathrm{N}+\mathrm{Ko})$ nachbehandelten Kohlevarianten weisen geringere Werte als die der puren Kohlevarianten auf. Ein Verdünnungseffekt ist aufgrund der geringeren Jahrestrieblängen der Kiefern dieser Varianten auszuschließen. Zwischen den Varianten bestehen in den Stickstoffgehalten der letztjährigen Nadeln und der Feinwurzeln nur wenig Unterschiede. Der letztjährige Nadeljahrgang der Kiefern der stickstoff$(+\mathrm{N})$ und kompost- $(+\mathrm{N}+\mathrm{Ko})$ nachbehandelten Kohlevarianten weist tendenziell geringere Stickstoffgehalte auf. Bei gleicher Stickstoffversorgung im Pflanzbeet der Baumschule ist von einem Abtransport des Stickstoffes aus dem letztjährigen Nadeljahrgang auszugehen. 


\subsubsection{Nährstoffverhältnisse}

In Tabelle A.5 im Anhang sind die Blatt/Nadel-Elementverhältnisse für Hainbuche und Kiefer dargestellt. Die festgestellten Abweichungen vom harmonischen Elementverhältnis bewegen sich mit Ausnahme des N/P-Verhältnis der Varianten Null und HKpur im Topfversuch stets im Bereich der zu geringen relativen Stickstoffkonzentration bzw. zu hohen relativen Nährkationenkonzentration (P, K, Ca, Mg).

Für die Kiefer lassen sich kaum unharmonische Elementverhältnisse nach Mellert und Göttlein (2012) feststellen. Lediglich das Verhältnis N/Ca ist im Jahr 2015 bei den Varianten Null und $\mathrm{HK} / \mathrm{SK}+\mathrm{N}+\mathrm{Ko}$ durch zu hohe relative Calciumgehalte gestört.

Für die Hainbuche lässt sich mit zunehmender Versuchsdauer ein unharmonisches N/P- und N/KVerhältnis bei den stickstoff- $(+\mathrm{N})$ und kompost- $(+\mathrm{N}+\mathrm{Ko})$ nachbehandelten Kohlevarianten hinsichtlich einer zu geringen relativen Stickstoffkonzentration beobachten. Diese Versuchsvarianten scheinen eine reduzierte Stickstoffverfügbarkeit aufzuweisen. Dies bekräftigt die Ergebnisse der Stickstoffblattgehalte dieser Varianten (2016 im Mangelbereich nach Krauß und Heinsdorf (2005)). Das Elementverhältnis N/Mg weist in fast allen Versuchsvarianten deutliche Abweichungen nach unten auf. Die Stickstoffverfügbarkeit scheint zu Gunsten der Magnesiumverfügbarkeit reduziert zu sein. 


\subsubsection{Zusammenfassung Ernährung}

Tabelle 3.13: Zusammenfassung Stickstoffernährung für Hainbuche

\begin{tabular}{cccc}
\hline Variante & 2014 & 2015 & 2016 \\
\hline Null & - & o & o \\
HKpur & - & o & - \\
SKpur & - & - & - \\
HK+N & ++ & o/- & - \\
SK+N & ++ & o/- & - \\
HK $+\mathrm{N}+\mathrm{Ko}$ & + & o/- & - \\
SK+N+Ko & + & o/- & - \\
\hline
\end{tabular}

Die Stickstoffgehalte der Hainbuchenblätter und der Feinwurzeln nehmen im Feldversuch im Jahresvergleich 2015/2016 in allen Versuchsvarianten stark ab. Die Nullvariante zeigt dabei die geringste Abnahme. Während im Jahr 2014 bei den Hainbuchen der stickstoff- $(+\mathrm{N})$ und kompost$(+\mathrm{N}+\mathrm{Ko})$ nachbehandelten Kohlevarianten von einer Überversorgung mit Stickstoff angenommen werden kann, ist die Stickstoffverfügbarkeit in diesen Varianten in den Folgejahren deutlich herabgesetzt (siehe Tab. 3.13).

Zwei Jahre nach Kohleeinarbeitung zeigen die Hainbuchen in allen Kohlevarianten eine schlechtere (mangelhafte) Stickstoffversorgung als in der Nullvariante. Die Entwicklung der SPAD-Werte zeigt in den Jahren 2015 und 2016 das gleiche Muster. Ungleichgewichte im N/Mg, N/K- und N/P-Verhältnis weisen in Richtung zu geringer relativer Stickstoffgehalte.

Die Hainbuchen der puren Kohlen- und Nullvarianten zeigen in der ersten Vegetationsperiode eine mangelhafte, die Hainbuchen der stickstoff- $(+\mathrm{N})$ und kompost- $(+\mathrm{N}+\mathrm{Ko})$ nachbehandelten Kohlevarianten eine optimale Stickstoffversorgung. Dieses Muster zeigt sich auch in der Calciumversorgung (Abbildung im Anhang A.5).

Die Hainbuchen der puren Holzkohlevariante zeigen höhere Stickstoffkonzentrationen in den Blättern und in den Feinwurzeln als die Hainbuchen der puren Spelzenkohlevariante (SKpur).

Dagegen weisen die Kiefern der puren Spelzenkohlevariante die höchsten Stickstoffkonzentrationen in den diesjährigen Nadeln auf. Die Kiefern der stickstoff- $(+\mathrm{N})$ und kompost- $(+\mathrm{N}+\mathrm{Ko})$ nachbehandelten Kohlevarianten zeigen leicht reduzierte Stickstoffgehalte in den dies- und letztjährigen Nadeln. Dies deutet auf ein Defizit in der Stickstoffversorgung.

Die Pflanzen beider Baumarten der Spelzenkohlevarianten (SK) weisen höhere Kaliumgehalte als die Pflanzen der entsprechenden Holzkohlevarianten (HK) gleicher Nachbehandlung auf (Abbildungen im Anhang A.7 bzw. A.10).

Die Hainbuchen und Kiefern der kompostnachbehandelten $(+\mathrm{N}+\mathrm{Ko})$ Kohlevarianten zeigen im Vergleich zu den Hainbuchen der stickstoffnachbehandelten $(+\mathrm{N})$ Kohlevarianten zum Teil reduzierte Kalium- und Phosphorgehalte (Abbildungen im Anhang A.7, A.8 bzw. A.10). 


\subsubsection{Physiologische Indikatoren}

\subsubsection{Blattwassergehalt und stomatäre Leitfähigkeit}
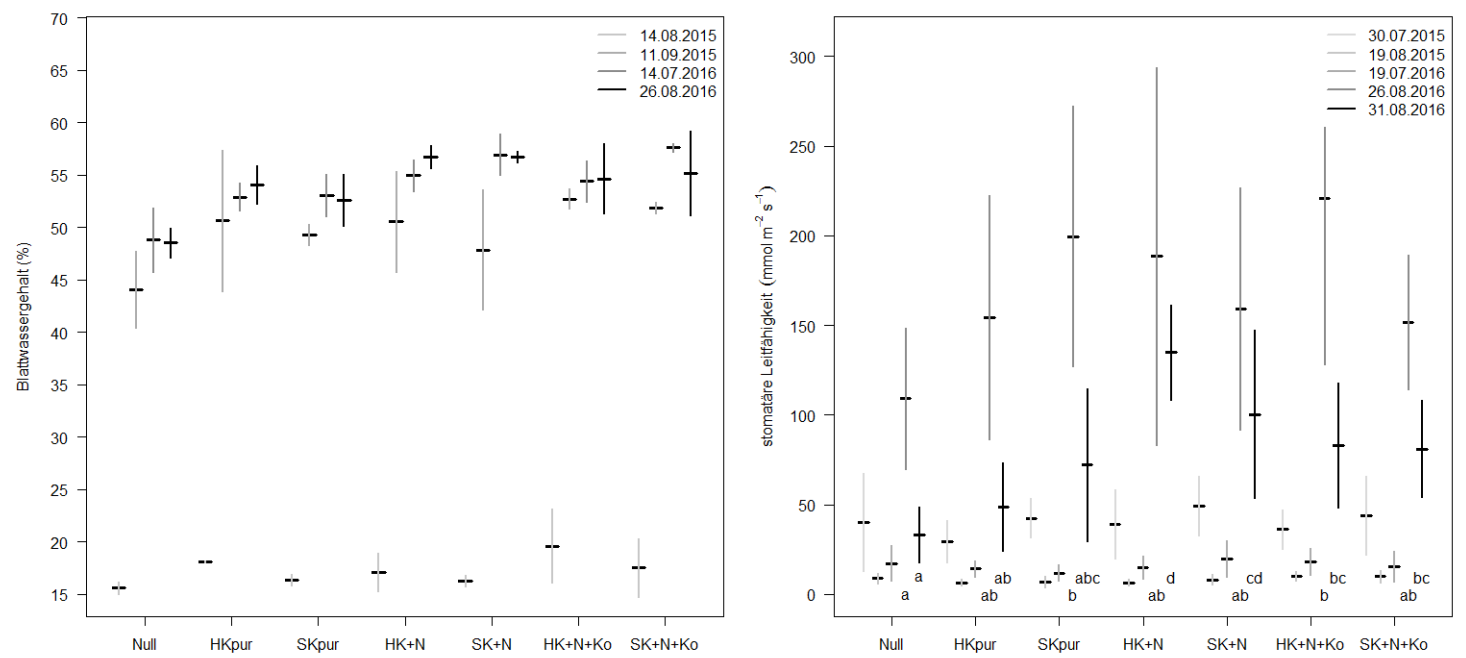

Abbildung 3.29: Blattwassergehalte (links) und stomatäre Leitfähigkeit der Hainbuchenblätter während der Vegetationszeiten 2015 und 2016. Mittelwerte und Standardabweichung. Unterschiedliche Buchstaben bezeichnen signifikante Unterschiede (Signifikanzniveau $\mathrm{p}<0,05 ; \mathrm{n}=3$ )

Die Blattwassergehalte der Hainbuchenblätter liegen am 14.08.2015 deutlich unter denen der übrigen Termine (siehe Abb. 3.29 (links)). Die höchsten Blattwassergehalte konnten am 26.08.2016 beobachtet werden. Die Wassergehalte der Hainbuchenblätter der Nullvariante liegen an allen Messterminen unter denen der Kohlevarianten.

Die Messergebnisse der stomatären Leitfähigkeit der Hainbuchenblätter im Jahr 2015 und im Juli 2016 im Feldversuch liegen in einem niedrigen Bereich und weisen damit auf einen verminderten Wasserdampftransport durch die Stomataöffnungen hin (siehe Abb. 3.29 (rechts)). Es bestehen an diesen Terminen kaum Unterschiede zwischen den Versuchsvarianten. An den letzten beiden Messterminen im August 2016 sind zum Teil deutlich höhere Leitfähigkeiten gemessen worden. Die Hainbuchen in den Kohlevarianten weisen dabei durchweg höhere stomatäre Leitfähigkeiten als die Hainbuchen der Nullvariante auf. Vor allem die Hainbuchen der stickstoff- $(+N)$ und kompost- $(+\mathrm{N}+\mathrm{Ko})$ nachbehandelten Kohlevarianten scheinen eine weitgehend unverminderte Photosynthese mit geöffneten Stomataöffnungen zu betreiben. 


\subsection{Zusammenfassung der wichtigsten Ergebnisse}

\subsubsection{Zeitliche Skalierung der Indikatoren und Effekte}

Die Ergebnisse des Feld-, Topf- und Extraktionsversuches machen eine zeitliche Einordnung der Wirkungen und Effekte, die auf die Einarbeitung von Biokohlen zurückzuführen sind, möglich. Sie lassen sich in folgende Kategorien einordnen:

- Die kurzfristigen Wirkungen auf die Pflanzen halten bis zum Ende der jeweiligen Trockenperiode an und sind meist reversibel. Die Effekte auf den Boden stehen ab dem Zeitpunkt der Kohleeinarbeitung zur Verfügung.

- Die mittelfristigen Wirkungen und Effekte sind innerhalb einer Vegetationszeit zu beobachten bzw. dauern eine Vegetationsperiode an.

- Die langfristigen Wirkungen und Effekte bilden sich erst in der folgenden Vegetationszeit aus und überdauern die zweite Vegetationszeit.

Tabelle 3.14: Zeitliche Skalierung der Auswirkungen auf Boden und Pflanze.

\begin{tabular}{|c|c|c|c|}
\hline Indikatoren und Effekte & kurzfristig & mittelfristig & langfristig \\
\hline \multicolumn{4}{|l|}{ Boden } \\
\hline Trockenrohdichte & $\mathrm{x}$ & $\mathrm{x}$ & $\mathrm{x}$ \\
\hline Bodenphysik (Porenvol., Textur) & $\mathrm{x}$ & $\mathrm{x}$ & $\mathrm{x}$ \\
\hline Sickerwassermenge in $90 \mathrm{~cm}$ Bodentiefe & $\mathrm{x}$ & $\mathrm{x}$ & $\mathrm{x}$ \\
\hline Hydrophobie & $\mathrm{x}$ & $\mathrm{x}$ & $\mathrm{x}$ \\
\hline gravimetrischer Wassergehalt & & $\mathrm{x}$ & $\mathrm{x}$ \\
\hline $\mathrm{nFK}$ & & $\mathrm{x}$ & $\mathrm{x}$ \\
\hline Bodenchemie (C- und N-Vorrat) & $\mathrm{x}$ & $\mathrm{x}$ & $\mathrm{x}$ \\
\hline Sickerwasserchemie $\left(\mathrm{NO}_{3}, \mathrm{NH}_{4}\right)$ & $\mathrm{x}$ & $\mathrm{x}$ & $\mathrm{x}$ \\
\hline \multicolumn{4}{|l|}{ Pflanze } \\
\hline Überlebenszahlen (Mortalität) & $\mathrm{x}$ & $\mathrm{x}$ & $\mathrm{x}$ \\
\hline Blattwassergehalt & $\mathrm{x}$ & $\mathrm{x}$ & $\mathrm{x}$ \\
\hline stomatäre Leitfähigkeit & $\mathrm{x}$ & $\mathrm{x}$ & $\mathrm{x}$ \\
\hline Blatt-Nadelverlust & $\mathrm{x}$ & $\mathrm{x}$ & $\mathrm{x}$ \\
\hline Deckungsgrad Begleitvegetation & & $\mathrm{x}$ & $\mathrm{x}$ \\
\hline Blattelementgehalte, SPAD-Wert & & $\mathrm{x}$ & $\mathrm{x}$ \\
\hline Blattfläche & & $\mathrm{x}$ & $\mathrm{x}$ \\
\hline Blatttrockenmasse & & $\mathrm{x}$ & $\mathrm{x}$ \\
\hline Zuwachs Wurzelhalsdurchmesser & & $\mathrm{x}$ & $\mathrm{x}$ \\
\hline Jahrestrieblänge Kiefer & & $\mathrm{x}$ & $\mathrm{x}$ \\
\hline Trockenmasse Feinwurzel & & $\mathrm{x}$ & $\mathrm{x}$ \\
\hline Mykorrhizierung & & $\mathrm{x}$ & $\mathrm{x}$ \\
\hline trockene Wurzelspitzen & $\mathrm{x}$ & $\mathrm{x}$ & $\mathrm{x}$ \\
\hline
\end{tabular}




\subsection{2 Übersicht über die Hauptergebnisse}

Es werden eindeutige Effekte aufgeführt, die in Feld,- Topf- und Extraktionsversuch ähnliche Muster aufweisen.

Tabelle 3.15: Übersichtsliste über Effekte im Vergleich zur Nullvariante. ++: viel höher; +: höher; o: neutral; -: kleiner; - -: viel kleiner; x: keine Aussage möglich. +N: stickstoffbehandelte Kohlevarianten; $+\mathrm{N}+\mathrm{Ko}$ : stickstoff- und kompostbehandelte Kohlevarianten. $\mathrm{HBU}=$ Hainbuche, $\mathrm{KI}=$ Kiefer.

\begin{tabular}{lllll}
\hline Indikatoren und Effekte & Zeitskala & pure Kohlen & $+\mathrm{N}$ & N + Ko
\end{tabular}

Wirkung von Bodenbearbeitung und Kohleneintrag auf bodenphysikalische Eigenschaften

Trockenrohdichte $(0-20 \mathrm{~cm})$

grav. Wassergehalt $30 \mathrm{~cm}$

grav. Wassergehalt $90 \mathrm{~cm}$

nutzbare Feldkapazität

Sickerwassermenge $90 \mathrm{~cm}$ langfristig

langfristig

langfristig

langfristig

langfristig

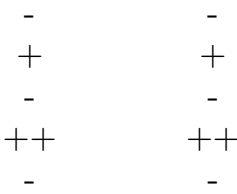

$\mathrm{o} /+$
$\mathrm{o}$
+
$\mathrm{o} /-$
-
$\mathrm{o} /+$

$++$

$-$

$++$

$+/++$

-

o

3. Jahr

1. Jahr

2. Jahr

3/4. Jahr

1. Jahr

2. Jahr

3/4. Jahr

1-4. Jahr

1/2. Jahr

2/3. Jahr

2. Jahr.

1. Jahr

1-3. Jahr

1. Jahr

1-4. Jahr

3. Jahr

3. Jahr

3. Jahr

3. Jahr

2/3. Jahr

2/3. Jahr

1-3. Jahr

$\mathrm{o}$
$\mathrm{o} /-$
$\mathrm{o} /+$
$\mathrm{o}$
$\mathrm{o} /-$
$\mathrm{o}$
$\mathrm{o}$
$\mathrm{o}$
$\mathrm{o}$
+
$\mathrm{o}$
$\mathrm{o} /+$
+
$\mathrm{o} /+$
$\mathrm{o}$
$\mathrm{o}$
-
-
+
+
++

$+$

-

$++$

trockene Wurzelspitzen KI

Stomatäre Leitfähigkeit

Blattwassergehalt HBU

Kaliumgehalt Blatt HBU 


\section{Diskussion}

\subsection{Einfluss von Biokohle auf Bodenphysik und Wasserhaushalt einschließlich Folgen der Bodenbearbeitung}

\subsubsection{Bodentextur}

Die Korngrößenanalysen in dieser Untersuchung zeigen, dass die Einbringung von Biokohle in einer Menge von 80 t ha ${ }^{-1}$ bzw. 2,8 Masse-\% die Textur des Feldbodens nur minimal verändert hat (vgl. Anhang A.1).

Die Biokohleteilchen können als Pseudokörner zwar Teil der Korngrößenzusammensetzung der Böden sein und die bodenphysikalischen Eigenschaften wie mineralische Partikel gleicher Korngröße beeinflussen (Downie et al. 2009), aufgrund der geringen Masseanteile der Kohlenzugabe sind jedoch nur sehr geringe Veränderungen in der Korngrößenverteilung zu erwarten (Haubold-Rosar et al. 2014). Langfristige Entwicklungen, durch die physikalische (Frost-TauZyklen, Austrocknung-Wiederbefeuchtung, Bioturbation), chemische oder biologische Zersetzung der Kohlen können noch nicht abgeschätzt werden (Hammes und Schmidt 2009; Lehmann und Joseph 2009).

\subsubsection{Trockenrohdichte}

Im Feldversuch führt das Mulchen des Oberbodens und das Fräsen im Zuge der Versuchsvorbereitung zu einer Auflockerung und Sauerstoffzufuhr in tiefere Schichten. Dadurch wird die Trockenrohdichte in den oberen $20 \mathrm{~cm}$ deutlich herabgesetzt. Zusätzlich kann der, durch die Bodenbearbeitung (Mulchen, Fräsen) in den Oberboden eingearbeitete Auflagehumus aufgrund des hohen Anteils organischer Biomasse zu einer Senkung der Trockenrohdichte (Scheffer 2002) führen. Auch zwei Jahre nach Bodenbearbeitung hält der Prozess der Bodensetzung noch an (siehe Abb. 4.1). Die typische mittlere Trockenrohdichte in den oberen $20 \mathrm{~cm}$ eines Sandbodens beträgt zwischen 1,1 und 1,2 $\mathrm{g} \mathrm{cm}^{-3}$ (Standortskartierung 2003; Paar et al. 2016).

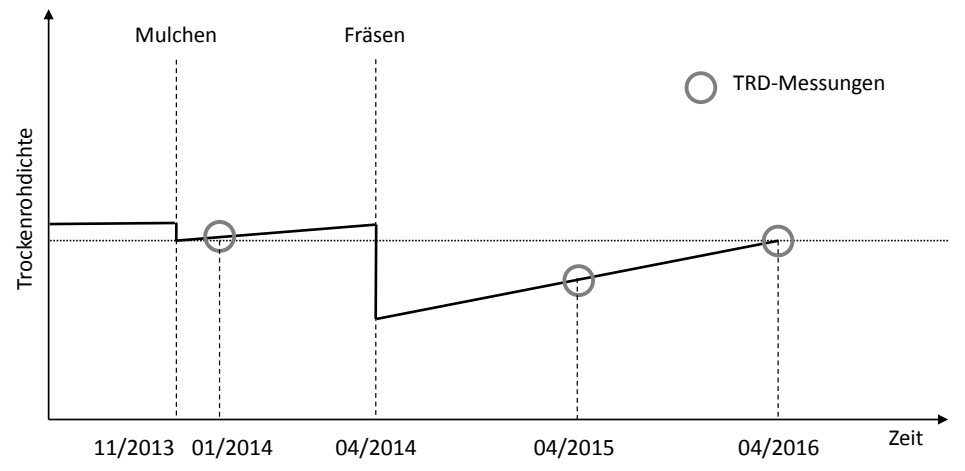

Abbildung 4.1: Entwicklung der Trockenrohdichte im Oberboden $(0-20 \mathrm{~cm})$ der Nullvariante nach Bodenbearbeitung in den Jahren 2013 - 2016. 
Die eingebrachten Kohlen mit ihren Trockenrohdichten zwischen 0,21 und 0,55 $\mathrm{g} \mathrm{cm}^{-3}$ tragen zusätzlich zur Senkung der Trockenrohdichte in den oberen $20 \mathrm{~cm}$ des sandigen Bodensubstrates um $0,1-0,5 \mathrm{~g} \mathrm{~cm}^{-3}$ bei.

Dieser Befund bestätigt Ergebnisse von Downie et al. (2009) und Abel et al. (2013). Auch Karhu et al. (2011), Revell (2011) und Berihun et al. (2017) konnten Rückgänge in der Trockenrohdichte zwischen 0,13 und $0,5 \mathrm{~g} \mathrm{~cm}^{-3}$ nach Kohleeinarbeitung in den Oberboden beobachten. Bereits geringe Eintragsmengen an Biokohle (1,5 Masse-\%) können die Trockenrohdichte eines sandigen Lehms deutlich absenken, zusätzliche Kohlemengen senken die Trockenrohdichte dagegen nur noch geringfügig weiter ab (Rogovska et al. 2011; Devereux et al. 2012).

Das bei den puren Kohlevarianten (HKpur und SKpur) beobachtete weitere Absinken der Trockenrohdichte in den kohlebeeinflussten Bodenschichten wird durch Ergebnisse von Burrell et al. (2016) bestätigt. Diese anhaltende Wirkung der Biokohle könnte nach Laird et al. (2010) durch eine erhöhte mikro- und makrobielle Aktivität auftreten, die durch die kohlebedingt gleichmäßigere Bodenfeuchte gefördert wird (Lei und Zhang 2013). Diese indirekte Wirkung von Biokohle trägt möglicherweise zu einer verbesserten Bodenstruktur und einer höheren Aggregatstabilität bei. Am Standort des Feldversuches kann die Kombination von Biokohlen-Kohlenstoff mit den kalkhaltigen Mineralphasen langfristig zur Bildung von Makroaggregaten führen (Herath et al. 2013; Lu et al. 2014).

\subsubsection{Wasserspeicherfähigkeit}

Die Retentionskurven der Versuchsvarianten zeigen durch die Kohleneinarbeitung eine Erhöhung des Porenvolumens der Mittel- bis Feinporen im Bereich von 0,2 - $50 \mu \mathrm{m}$. In reinen Sandböden dominieren die Grobporen bei der Wasserspeicherung. Damit steht durch den Kohleeintrag grundsätzlich mehr Raum für pflanzenverfügbares Kapillar- und Haftwasser zur Verfügung. Die Feldkapazität ist durch die Kohlebeimischung im Oberboden um 13 bzw. 20 Vol.-\% (mm) gestiegen.

Luft- und Wasserhaushalt eines Bodens können durch größere Gesamtporenvolumen grundsätzlich positiv beeinflusst werden. Zur Bewertung des Wasserhaushaltes eines Bodens können Wasserhaltekapazität und pflanzennutzbare Feldkapazität (nFK) als Kriterien herangezogen werden. Beide Größen sind stark von Bodenart, Trockenrohdichte des Feinbodens, des Grobbodenanteils und des Humusgehalts abhängig (Standortskartierung 2003). Die Ergebnisse dieser Untersuchung zeigen, dass sich die Faktoren Bodenart und Grobbodenanteil (0 \%) im Versuchszeitraum durch die Kohleneinmischung kaum verändert haben. Durch das Mulchen und Fräsen des Oberbodens im Zuge der Versuchsvorbereitung wurden die mächtige Humusauflage (rohhumusartiger Moder) und kleine Teile der Strauchvegetation in den Oberboden eingearbeitet. Diese Biomasse besitzt positive Wirkungen auf die Wasserspeicherfähigkeit des Oberbodens, ebenso die Lockerung des Oberbodens durch die Bearbeitung.

Für den kohleunbehandelten Boden der Nullvariante wurde ein nFK-Wert von 148 mm berechnet. Dieser Wert liegt im oberen Bereich der Substratgruppe „unverlehmte Sande“ in der zweiten Bodenzustandserhebung, für die mittlere nFK-Werte von etwa $140 \mathrm{~mm}$ bis maximal 90 cm Bodentiefe berechnet wurden (Paar et al. 2016). Nach der Bewertung der Forstlichen Standortsaufnahme (Standortskartierung 2003) entspricht dieser Wert einem relativ hohen Bereich der nutzbaren Feldkapazität.

Die Biokohle zeigt in dieser Untersuchung einen positiven Effekt auf die pflanzennutzbare Feldkapazität im Oberboden. Für die Kohlevarianten HKpur bzw. HK+N+Ko kann im Oberboden (0 $25 \mathrm{~cm}$ ) eine Erhöhung um 9,3 bzw. 14,2 Vol.-\% (49 \% bzw. 75 \%) festgestellt werden. Kammann et al. (2011) haben in ihren Untersuchungen bei Kohlezugaben von 100 bzw. 200 t ha ${ }^{-1}$ bei einem 
reinen Sandboden bis zu 24 bzw. 36 \% erhöhte Wasserhaltekapazitäten ermittelt. Auch Novak et al. (2012) und Basso et al. (2013) haben in ihren Experimenten eine Erhöhung der Wasserhaltekapazität um 15 - $23 \%$ durch Biokohlenbeimischung zu sandigem Lehm als Ausgangssubstrat beobachten können.

Briggs et al. (2005) konnten bei einem sandigen Lehm durch Zugabe von 5 Massen-\% Holzkohle eine Erhöhung der nFK um 6,1 Vol.-\% beobachten. Glaser et al. (2002) konnten in Terra PretaBereichen in Amazonien eine um 18 \% höhere nFK feststellen als in umliegenden holzkohlefreien Bodenbereichen. Gegenteilige Effekte haben dagegen Devereux et al. (2012) für einen sandigen Lehm bei Kohlezugabe von 1,5 - 5 Masse- $\%$ beobachtet. Die niedrigere nutzbare Feldkapazität resultiert möglicherweise aus dem höheren Anteil von Feinporen, mit höherem Anteil nicht pflanzenverfügbaren Totwassers.

Das Ausgangssubstrat „unverlehmter Sand“ hat einen durchschnittlichen Tongehalt von unter $4 \%$. Je geringer der Tongehalt des Ausgangssubstrates, umso größer kann der Einfluss von Biokohle auf die hydrologischen Bodeneigenschaften sein (Castellini et al. 2015). Abel et al. (2013) stellten bei Zugabe von Biokohle in zwei Versuchsböden fest, dass die Erhöhung der nFK bei einem sandigen Boden deutlich größer ist als bei einem lehmigen Boden. Bei einem tonreichen Boden konnten Major et al. (2010) trotz Zugabe von bis zu $20 \mathrm{t} \mathrm{ha}^{-1}$ keinen Effekt der Kohle auf die Wasserspeicherfähigkeit feststellen.

Der kohleunbeeinflusste Unterboden $(25-90 \mathrm{~cm}$ Bodentiefe) hat jedoch rechnerisch ebenfalls einen großen Einfluss auf die nFK-Summe, daher erhöht sich die nutzbare Feldkapazität bis 90 cm Bodentiefe um 16,3\% bzw. 24,9\%. Diese Werte übersteigen das anvisierte Versuchsziel, einer $15 \%$-Verbesserung der nutzbaren Feldkapazität. Für die Wasserversorgung der Pflanzen ist jedoch der Füllstand der nFK zu Beginn der Vegetationszeit entscheidend. Trockene Nichtvegetationszeiten können dazu führen, dass den Pflanzen zu Beginn der Vegetationszeit kein maximal aufgefüllter Bodenwasserspeicher zur Verfügung steht und somit ein erhöhtes Risiko für eine angespannte Wasserversorgung bestehen kann. Dies war zu Beginn der Vegetationszeiten 2014 und 2017 der Fall.

\subsubsection{Hydrophobie der Bodenoberfläche}

Die gemessenen Versickerungszeiten im Feldversuch während der Vegetationsperiode 2015 waren für einen Sandboden unerwartet hoch und stiegen mit anhaltender Trockenheit bis auf über 250 s $100 \mathrm{ml}^{-1}$ an. Steubing und Fangmeier (1992) geben für einen Sandboden eine Versickerungszeit

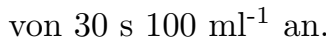

Die Benetzbarkeit des Bodens beeinflusst wichtige hydraulische Bodeneigenschaften wie die Wasserretention und die Wasserleitfähigkeit (Bachmann et al. 2016). Eine reduzierte Benetzbarkeit führt zu einer geringeren Wasserspeicherung, da das Wasser nicht ungehindert infiltrieren kann (Buczko et al. 2002). Weiterhin fördert eine herabgesetzte Benetzbarkeit den Oberflächenabfluss, die Bodenerosion und die Ausbildung von präferentiellen Fließwegen im Bodenkörper (Dekker und Ritsema 1994; Bauters et al. 2000). Mit höheren Oberflächenabfluss- und geringeren Inflitrationsmengen sinkt das pflanzenverfügbare Wasserangebot während der Vegetationszeit.

In diesem Versuch haben sich durch die Kohleeinarbeitung die Gehalte an organischem Kohlenstoff im Oberboden im Vergleich zur Nullvariante mehr als verdoppelt. Die Nullvariante weist $1,2 \%$, die Kohlevarianten im Mittel $3 \%$ organischen Kohlenstoffs auf.

Einen Zusammenhang zwischen Benetzungshemmung und dem Gehalt an organischen Kohlenstoff im Boden (SOC) haben mehrere Autoren festgestellt (Täumer et al. 2005; Mao et al. 2014). Auch Bachmann et al. (2016) haben eine positive Korrelation zwischen Benetzungshemmung und 
SOC-Gehalt ab einem Grenzwert von 0,3\% SOC gezeigt. Die Biokohle mit ihren hohen Kohlenstoffanteilen kann je nach Ausgangssubstrat und Pyrolysebedingungen ebenfalls hydrophobe Eigenschaften aufweisen (Kinney et al. 2012), die die Infiltrationsraten des Oberbodens negativ beeinflussen können (Doerr et al. 2000; Robichaud et al. 2008). Bei Laboruntersuchungen von Page-Dumroese et al. (2015) sind bei einer zugegebenen Kohlenmenge von $10 \mathrm{t} \mathrm{ha}{ }^{-1}$, gerade bei geringen Bodenwassergehalten, deutlich reduzierte Infiltrationsraten und erhöhte Infiltrationszeiten beobachtet worden.

Atkinson et al. (2010) zeigen, dass die hydrophobe Eigenschaft der Biokohlen zunächst überwunden werden muss, damit die erwünschten Wirkungen (Wasserabsorption und -retetention) erreicht werden können. Wallis et al. (1991) und Viviani und Iovino (2004) weisen darauf hin, das selbst nach vollständiger Durchfeuchtung die Infiltrationsraten von hydrophoben Böden nur etwa $10-50 \%$ von hydrophilen Böden betragen können. Bei sandigen Böden konnten Abel et al. (2013) dagegen keinen Einfluss von Biokohle auf die Durchfeuchtbarkeit von Böden nachweisen. Durch andauernde Trockenperioden können sich jedoch wieder hydrophobe Eigenschaften ausbilden und die Benetzbarkeit der Bodenoberfläche herabsetzen (Gimbel et al. 2016). 


\subsection{Einfluss von Biokohle auf den Stickstoffhaushalt}

Folgende Prozesse können aufgrund der Bodenbearbeitung zur Versuchsvorbereitung und der Kohleneinarbeitung ausgeschieden werden:

1. Mulchen: Bodenlockerung, Stickstoffmineralisierung

2. Fräsen: Bodenlockerung, Stickstoffmineralisierung

3. Kohleeinarbeitung:

a) kurzfristig: Stickstoffeintrag, -mineralisierung, -immobilisierung, -austrag

b) langfristig: Stickstoffimmobilisierung

\subsubsection{Einfluss der Bodenbearbeitung auf die Stickstoffmineralisierung}

Der Verlauf der Stickstoffausträge der Nullvariante kann als Indikator für die durch die Bodenbearbeitung Ende 2013 und im April 2014 angeregte Mineralisierung interpretiert werden. Die akkumulierten Humusmengen und die Wurzeln des geernteten Bestandes stehen als Ausgangsmaterial für die Zersetzung und Mineralisierung zur Verfügung. Noch Anfang des Jahres 2015 sind erhöhte Werte von Nitrat und Ammonium im Sickerwasser nachzuweisen, bevor sich die Werte ab Mitte 2015 stabilisieren. Es kann daher von erhöhten Mineralisierungsraten vom Zeitpunkt der Bodenbearbeitung bis etwa Mitte 2015 ausgegangen werden.

Prozesse der Stickstoffmineralisierung werden durch biotische Faktoren wie dem organischen Stickstoffanteil im Boden, der Aktivität der Bodenfauna und der Struktur der mikrobiellen Gemeinschaft und der Mykorrhiza im Boden beeinflusst. Auch abiotische Faktoren wie Bodentemperatur, -feuchtigkeit, -durchlüftung und -struktur bestimmen die aktuellen Mineralisationsrate eines Boden (Nadelhoffer et al. 1991; Knoepp und Vose 2007).

\subsubsection{Stickstoffeintrag durch die Biokohlen}

In diesem Versuch wurden Kohlen verwendet, die aus dem Ausgangsmaterialien Holzkohle bzw. Getreidespelzen mittels Pyrolyse bei Temperaturen über $650{ }^{\circ} \mathrm{C}$ hergestellt wurden. Die Nachbehandlung der Stickstoff- $(+\mathrm{N})$ und Kompost- $(+\mathrm{N}+\mathrm{Ko})$ Kohlevarianten mit Ammoniumsulfat hat einen Stickstoffeintrag dieser Kohlevarianten bis zu $1100 \mathrm{~kg} \mathrm{ha}^{-1}$ (siehe Tabelle 3.6, S. 51) ergeben. Dementsprechend ist ein enormer Einfluss auf den Stickstoffkreislauf des Ökosystems zu erwarten. In der Initialphase des Versuches (2014 und 2015) sind Stickstoffausträge aus dem System zu verzeichnen. In der zweiten Phase ab 2016 des Versuches überwiegen Prozesse der Stickstoffimmobilisierung. Dies zeigen die gewählten Indikatoren. Eine Zusammenfassung der Ergebnisse zum Umweltfaktor Stickstoff befindet sich auf Seite 62.

Die Kohle besitzt sowohl eine direkte Wirkung durch mit der Kohle zugeführte Nährstoffe, als auch eine indirekte Wirkung auf den Nährstoffhaushalt eines Bodens. Die indirekte Wirkung resultiert aus Veränderungen von Bodenreaktion, Sorptionskapazität, Wasserhaushalt (siehe Abschnitt 4.1) und mikrobieller Umsetzungsprozesse (sog. „Priming-Effekt“) (Haubold-Rosar et al. 2014).

Zunächst werden die chemischen Eigenschaften der eingebrachten Kohlen betrachtet (direkte Wirkung). Die Nährstoffgehalte der puren Kohlesubstrate werden vor allem durch die verwendeten Ausgangsstoffe und deren Elementgehalte bestimmt. Allgemein sind die Nährstoffpotenziale 
von puren Holzkohlen als vergleichsweise gering einzuschätzen. Diese Aussage gilt für alle Makronährstoffe mit Ausnahme von Kohlenstoff. Die hohen Kohlenstoff- bzw. niedrigen Stickstoffgehalte (weites C/N-Verhältnis) der puren Holzkohlesubstrate in unserem Versuch sind typisch für Kohlen aus holzigen Ausgangssubstraten (Haubold-Rosar et al. 2014).

Die puren Spelzenkohlen (SKpur) enthalten deutlich höhere Mengen an Stickstoff als die puren Holzkohlen (HKpur).

\subsubsection{Stickstoffmineralisierung, -immobilisierung und Stickstoffaustrag}

Der Stickstoffaustrag kann vereinfacht als eine Funktion der Mineralisierung, der Immobilisierung (Festlegung) und der Pflanzenaufnahme von Stickstoff aufgefasst werden. Im Extraktionsversuch können die Effekte der Mineralisierung und Festlegung ohne die Pflanzenaufnahme betrachtet werden. Eine konsequente Trennung der Effekte der Mineralisierung und Festlegung bzw. Immobilisierung ist jedoch kaum möglich. Eine zusammenfassende Übersicht über die Effekte von Stickstoffeintrag, Mineralisierung, Immobilisierung und Stickstoffverfügbarkeit in der zeitlichen Entwicklung gibt Tabelle 4.1 auf Seite 93.

Die im Feldversuch im Vergleich zur Nullvariante erhöhten Nitratausträge mit dem Sickerwasser der puren Kohlevarianten (HKpur bzw. SKpur, ohne Stickstoffbehandlung), die höheren Deckungsgrade und nitrophileren Arten in der Begleitvegetation geben Hinweise auf einen „PrimingEffekt" durch die Kohlen, mit Auswirkungen auf die Stickstoffmineralisierung im Oberboden. Biokohlen haben die Eigenschaft als Senke für zahlreiche chemische Verbindungen zu wirken und werden daher oft in der Filtertechnik eingesetzt (Aktivkohlefilter). Im Boden kann die Bindung von nitrifikationshemmenden Substanzen (Phenole u. a.) durch die Kohlen eine erhöhte mikrobielle Umsetzung der organischen Substanz zur Folge haben (Berglund et al. 2004; Wardle et al. 2008; Rogovska et al. 2011). Gundale und DeLuca (2006) beobachteten einen Anstieg der mikrobiellen Biomasse durch die Einarbeitung von Biokohlen. Dempster et al. (2012) haben in ihren Biokohlenversuchen eine um $25 \%$ geringere Nitratauswaschung aus sandigen Böden beobachtet und vermuten eine durch Biokohle herabgesetzte Nitrifikationsrate. Die Bedingungen auf dem Versuchsfeld sind durch einen hohen Boden-pH, eine gute Durchlüftung und einen für Mikroorganismen günstigen Temperaturverlauf im Oberboden gekennzeichnet. An der Ammonifikation, als erstem Schritt der Mineralisation, ist ein breites Sprektrum an heterotrophen, aeroben sowie anaeroben Mikroorganismen beteiligt (Carlyle 1986). Deshalb kann dieser Prozess in einem breiten Temperatur-, Sauerstoff- und pH-Bereich stattfinden (Rapp 1991). Die Nitrifikation von Ammonium zu Nitrat ist dagegen stark von Temperatur und $\mathrm{pH}$-Wert abhängig. Sie verläuft bei niedrigen pH-Werten stark gehemmt. Bei mittleren bis höheren pH-Werten, wie in dieser Untersuchung, wird ein Großteil des Ammonium innerhalb kurzer Zeit nitrifiziert (Hock 1988).

In dieser Arbeit kann eine enorme Fähigkeit der Festlegung von Stickstoff durch die Biokohlen festgestellt werden. Dies zeigt die enorme Saldomenge in der Stickstoffbilanz der kompostbehandelten Holzkohlevariante (HK+N+Ko) (siehe Abb. 4.4 auf S. 97). Auch die gemessenen mineralisierten Stickstoffmengen der puren Kohlevarianten liegen im Extraktionsversuch deutlich unter denen der Nullvariante, was auf eine Stickstoffimmobilisierung und eine möglicherweise verzögert beginnende Mineralisation schließen lässt. Dieser Befund wird durch die Ergebnisse der Sickerwasseranalysen des Topfversuches bestätigt. Die puren Kohlevarianten weisen im Topfversuch geringere Nitratkonzentrationen im Sickerwasser als die Nullvariante auf. Dies steht nur scheinbar im Widerspuch zu den Ergebnissen des Feldversuches. Topf- und Extraktionsversuch bilden den Zeitraum unmittelbar nach Kohleeinbringung ab. In dieser Phase ist bereits ein hohes Immobilisierungspotenzial zu beobachten Aufgrund der weiten $\mathrm{C} / \mathrm{N}-$ Verhältnisse der puren 


\begin{tabular}{|c|c|c|c|c|c|c|}
\hline 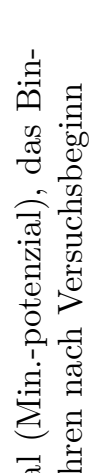 & 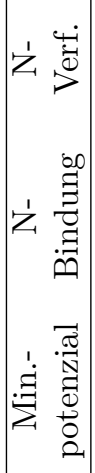 & 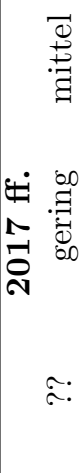 & 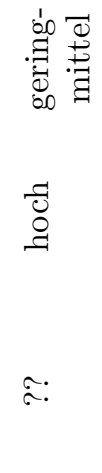 & 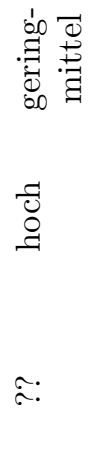 & 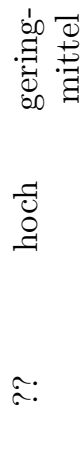 & 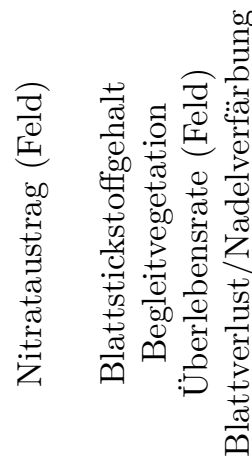 \\
\hline 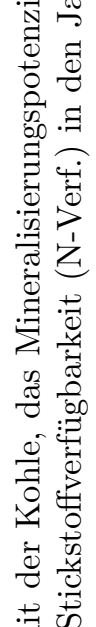 & 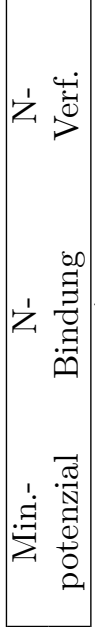 & 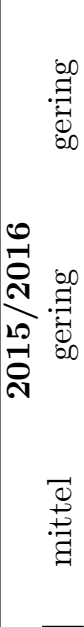 & 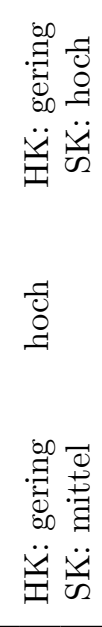 & 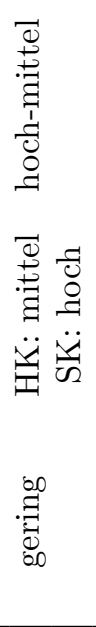 & 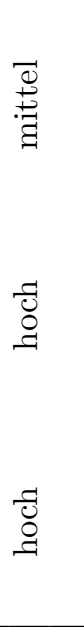 & 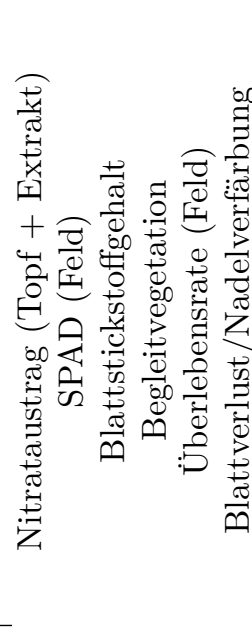 \\
\hline 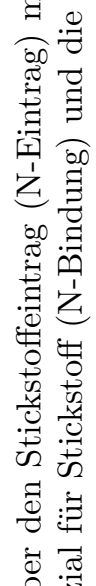 & 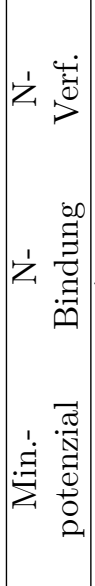 & 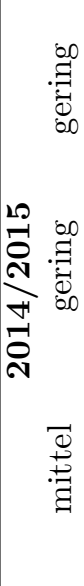 & 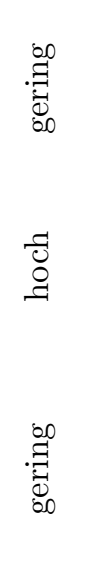 & 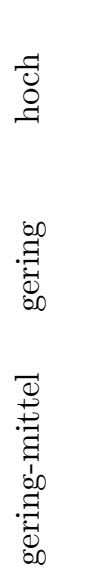 & $\begin{array}{l}\frac{1}{0} \\
0 \\
9 \\
\vdots \\
0 \\
0 \\
0\end{array}$ & 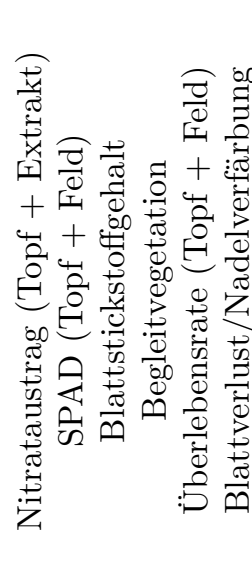 \\
\hline 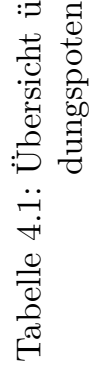 & 之 & $\begin{array}{l}\text { 三 } \\
\text { z }\end{array}$ & 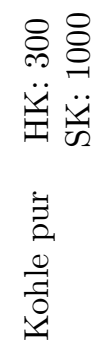 & $\begin{array}{l}8 \\
8 \\
1 \\
8 \\
8 \\
0\end{array}$ & $\begin{array}{l}0 \\
i \\
+ \\
z \\
+\end{array}$ & 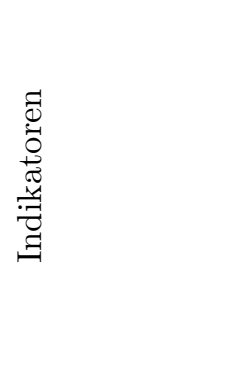 \\
\hline
\end{tabular}


Kohlen in Verbindung mit der fehlenden biologischen Aktivierung läuft die Mineralisierung nur gehemmt an. Innerhalb des ersten Jahres steigt das Mineralisierungspotenzial an, sodass sich die Stickstoffausträge der puren Kohlen denen der Nullvariante annähern und diese schließlich übersteigen, wie im Feldversuch zu beobachten ist. Das beschriebene Muster weist auf ein wirksames Potenzial zur Stickstofffestlegung der Kohlen und eine möglicherweise verminderte Pflanzenverfügbarkeit des Stickstoffs in der Anfangsphase des Feldversuches.

Die Pyrolysetemperaturen der eingesetzten Kohlen von über $400{ }^{\circ} \mathrm{C}$ können die resultierende Stickstoffverfügbarkeit für Pflanzen (Wang et al. 2012) verringern. Deshalb kann im landwirtschaftlichen Einsatz eine Stickstoffmangelversorgung auftreten und eine angepasste Stickstoffdüngung bei der Verwendung von Biokohlen erforderlich sein (Gaskin et al. 2008).

Bereits im dritten Jahr (2016) zeigen die Varianten der Stickstoff- $(+\mathrm{N})$ und kompostbehandelten Kohlen $(+\mathrm{N}+\mathrm{Ko})$ trotz eines enormen Stickstoffeintrags von bis zu $1133 \mathrm{~kg} \mathrm{ha}^{-1}$ und einer damit verbundenen bis zu 30 \%-igen Erhöhung des Stickstoffvorrates (Variante SK+N+Ko) meist geringere Stickstoffausträge als die Nullvariante ohne Stickstoffeintrag durch die Kohlen.

Amonette und Joseph (2009) und Downie et al. (2009) weisen auf die hohe Sorptionskapazität für Kationen hin, die aus der sehr großen inneren Oberfläche von Kohlen und den negativen Ladungsüberschüssen an diesen Oberflächen resultiert. Deshalb kann Ammonium besonders stark adsorbiert werden (Lehmann et al. 2006; Yao et al. 2012).

Die Ergebnisse der Sickerwasseruntersuchungen zeigen eindrücklich, dass grundsätzlich auch eine Nitratspeicherung bzw. eine verminderte Nitratauswaschung durch Biokohlen möglich ist, wie auch Mizuta et al. (2004) und Sika und Hardie (2014) in ihren Untersuchungen beobachtet haben. Voraussetzung ist nach Clough et al. (2013) eine Pyrolysetemperatur der Kohlen von über $600{ }^{\circ} \mathrm{C}$. Kameyama et al. (2012) verweisen auf eine schwache Adsorption von Nitrat an den Biokohlenoberflächen und vermuten eher eine physikalische Absorption des Nitrates in das Innere der Biokohlen ohne eine chemische Reaktion. Joseph et al. (2018) beschreiben eine komplexe Reaktionskette, die nach dem Transport der Ionen entlang eines Konzentrationsgradienten in das Innere der Kohlenporen in eine stabile Ablagerung an organomineralischen Belägen endet. Diese Beobachtung wird von Yao et al. (2012) gestützt, die an Biokohlen mit den größten inneren Oberflächen die stärkste Nitratabsorption beobachtet haben. Auch Borchard et al. (2012) haben den Zusammenhang von steigendem Nitratspeichervermögen bei höheren inneren Oberflächen von Biokohlen beschrieben.

Die Nachbehandlung der Stickstoff- $(+\mathrm{N})$ und kompostbehandelten Kohlen $(+\mathrm{N}+\mathrm{Ko})$ geschah durch das Besprühen mit Ammoniumsulfatlösung. Die dadurch eingebrachte Stickstoffmenge von bis zu $1133 \mathrm{~kg} \mathrm{ha}^{-1}$ lässt sich 2015 gut anhand der sehr hohen Stickstoffausträge mit dem Sickerwasser nachvollziehen. Die Stickstoffausträge folgen im Jahr 2015 keinem saisonalen Verlauf, das heisst auch während der Vegetationszeit sind hohe Nitratausträge zu beobachten. Während dieser Phase übersteigt das Stickstoffangebot die Pflanzenaufnahme und das Festlegungspotenzial im Boden deutlich. Bereits im Jahr 2016 liegen die Sickerwasserausträge von Stickstoff jedoch auf dem Niveau der Nullvariante.

Die Stickstofffraktion in der Bodenlösung besteht zu einem überwiegenden Teil aus Nitrat. Lediglich die Bodenlösung der Kohlenvariante HK $+\mathrm{N}$ des Herstellers Pyreg zeigt im Feldversuch bis 2016 höhere Ammoniumkonzentrationen. Die maximalen Ammoniumkonzentrationen im Sickerwasser dieser Kohlevariante beträgt in der Initialphase bis zu $200 \mathrm{mg} \mathrm{l}^{-1}$ (Topfversuch).

Ammonium und Nitrat unterscheiden sich stark in ihrer Mobilität im Boden. Das Ammoniumkation wird durch Austausch- und Fixierungsprozesse von Mikroorganismen und Mykorrhizapilzen sehr stark adsorbiert und kommt in der Bodenlösung meist nur in geringen Konzentrationen vor (Eichhorn 1995). Zudem besitzen die meisten Pflanzen eine starke Präferenz in der Auf- 
nahme von Ammonium als Stickstoffquelle (Carlyle 1986). Die hohe Mobilität des Nitrations bedingt eine rasche Verlagerung mit dem Sickerwasser in tiefere grundwasserleitende Bodenschichten (Hock 1988). Generell kann von einer raschen Umsetzung des freien Ammoniums in Nitrat ausgegangen werden, wie dies auch Meesenburg et al. (2004) nach einer ähnlich hohen Ammoniumsulfatdüngung in einem Buchenökosystem nachgewiesen haben. Die Austragsmengen von Ammonium betragen weniger als $10 \%$ von Nitrat. In einem stickstoffgesättigten Buchenökosystem hat Eichhorn (1995) einen Ammoniumgehalt von weniger als $5 \%$ des Nitratanteils beobachtet. In der Bodenlösung borealer und temperierter Wälder beobachteten Vitousek et al. (1982) Ammoniumkonzentrationen zwischen 7,2 und $72 \mathrm{mg} \mathrm{l}^{-1}$.

Die Varianten der kompostbehandelten Kohlen $(+\mathrm{N}+\mathrm{Ko})$ weisen im Topfversuch niedrigere Ammoniumausträge als die stickstoffbehandelten $(+\mathrm{N})$ Kohlen auf. Dies spricht für ein erhöhtes Potenzial zur Ammoniumadsorption durch den Prozess der Kompostierung.

Die Kompostierung der Kohlevarianten $(+\mathrm{N}+\mathrm{Ko})$ vor Einbringung hatte das Ziel, die Kohlen biologisch zu aktivieren und einen Alterungsprozess künstlich zu beschleunigen. Mit fortschreitender „Alterung“ der Kohlen können durch Oxidation der Kohleoberflächen die negativen Ladungsüberschüsse und somit die Kationenaustauschkapaziztät nochmals erhöht werden (Liang et al. 2006; Cheng et al. 2008).

Für die Stickstoff- $(+\mathrm{N})$ und kompostbehandelten Kohlen $(+\mathrm{N}+\mathrm{Ko})$ kann nach etwa drei Versuchsjahren ein sehr hohes Potenzial der Stickstofffestlegung durch die Kohlen bilanziert werden. Ob die Stickstoffspeicherung als Effekt der Nachbehandlung langfristig zur Verfügung steht, kann nach der 4-jährigen Versuchsdauer noch nicht abgeschätzt werden.

\subsubsection{Stickstoffflüsse und -vorräte}

Die Stickstoffflüsse und -vorräte in den Kompartimenten Bodenvorrat und Vegetation, die Einträge durch Kohleneinarbeitung, luftbürtige Deposition und Mineralisation sowie die Austräge durch das Sickerwasser bzw. $\mathrm{N}_{2} \mathrm{O}$-Emission sind für die Nullvariante und die Varianten HKpur und $\mathrm{HK}+\mathrm{N}+\mathrm{Ko}$ exemplarisch in den Abbildungen 4.2, 4.3 und 4.4 für die ersten drei Versuchsjahre dargestellt. Für die Sickerwasserausträge im Jahr 2014 liegen keine Daten vor, es wird jedoch ein mindestens gleichhoher Austrag wie 2015 angenommen. Die Stickstoffdeposition wird vom Umweltbundesamt (Schaap et al. 2015) für diesen Bereich im Zeitraum 2013 - 2015 mit 10 $\mathrm{kg} \mathrm{ha}^{-1} \mathrm{a}^{-1}$ angegeben.

$\mathrm{N}_{2} \mathrm{O}$-Emissionen werden vor allem durch Nitrifikations- und Denitrifikationsvorgänge von Stickstoffdüngern verursacht. Durch die Nachbehandlung der Kohlen mit Ammoniumsulfat und der Kompostierung können nach der Ausbringung Lachgasemissionen entstehen, die aber nach einigen Monaten abklingen (Wulf et al. 2003). Gemäß Solomon et al. (2007) sind Lachgasemissionen in Höhe von jährlich 1,25 \% des applizierten mineralischen Stickstoffes über einen Zeitraum von 6 Jahren zu erwarten. Hinsichtlich eines möglichen Minderungspotenzials von Lachgasemissionen durch Biokohle bestehen unterschiedliche Untersuchungsergebnisse. Nach Kohleneinarbeitung können sowohl erhöhte als auch verminderte Emissionen von Treibhausgasen wie Methan und Lachgas auftreten. Die zugrundeliegenden Wirkmechanismen sind noch nicht ausreichend bekannt (Haubold-Rosar et al. 2014). Für die Stickstoffbilanz in dieser Arbeit wird daher eine emissionsneutrale Wirkung der Kohle angenommen und eine Lachgasmenge von 1,25 \% der applizierten Stickstoffmenge bilanziert.

Bei der Gegenüberstellung von Ein- und Austrägen (Saldo) stellen alle drei Varianten in den ersten drei Jahren des Feldversuches eine Stickstoffsenke dar (positiver Saldo). Allerdings sind die angegebenen Mineralisations- bzw. Denitrifikationsraten nur grobe Schätzungen bzw. An- 
nahmen. Das Mineralisierungspotenzial der puren Kohlevarianten könnte, bedingt durch den „Priming-Effekt" höher liegen als bei der Nullvariante.

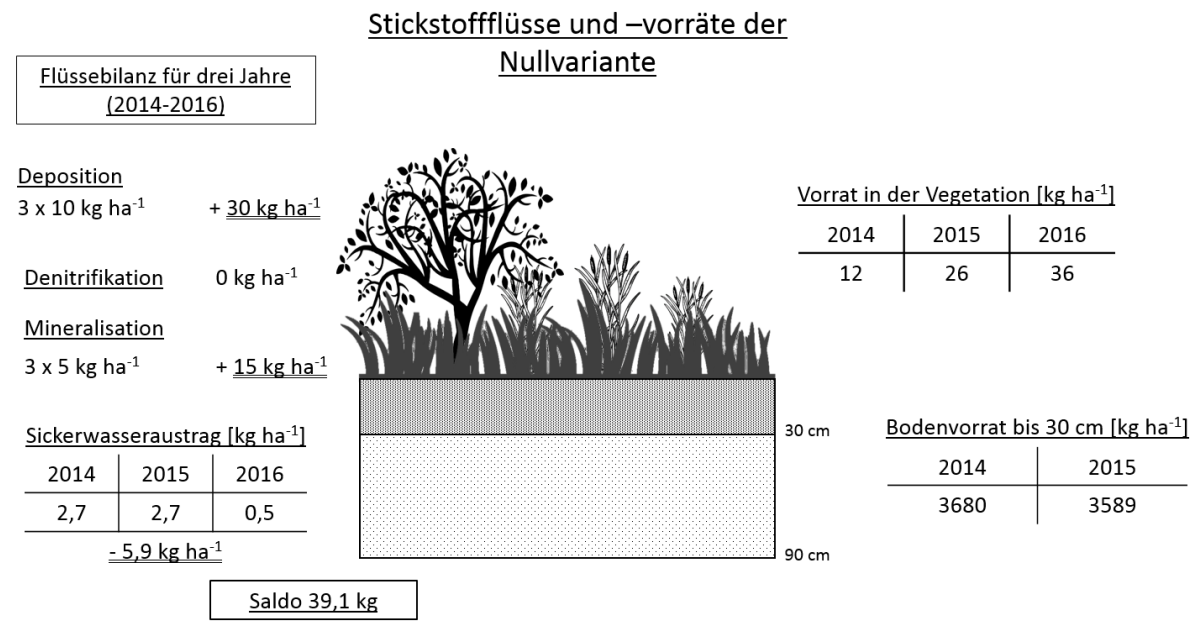

Abbildung 4.2: Stickstoffflüsse und -vorräte der Nullvariante in den Jahren 2014 - 2016.

Der Stickstoffvorrat vor Kohleeinarbeitung im Frühjahr 2014 betrug $3680 \mathrm{~kg} \mathrm{ha}^{-1}$ (bis $30 \mathrm{~cm}$ Bodentiefe). Mit der Kohle wurde im Jahr 2014 je nach Versuchsvariante zwischen 290 und 1133 $\mathrm{kg} \mathrm{ha}^{-1}$ Stickstoff eingetragen. Für die Variante HK+N+Ko beträgt der Saldo zwischen Ein- und Austrägen etwa $805 \mathrm{~kg} \mathrm{ha}^{-1}$. Die Differenz zwischen den Stickstoffvorräten 2014 und 2015 beträgt $690 \mathrm{~kg} \mathrm{ha}{ }^{-1}$. Damit weisen Saldo und Vorratsdifferenz eine weitgehende Übereinstimmung auf. Für die verbleibende Differenz ist wahrscheinlich die hohe räumliche Variabilität im Oberboden verantwortlich.

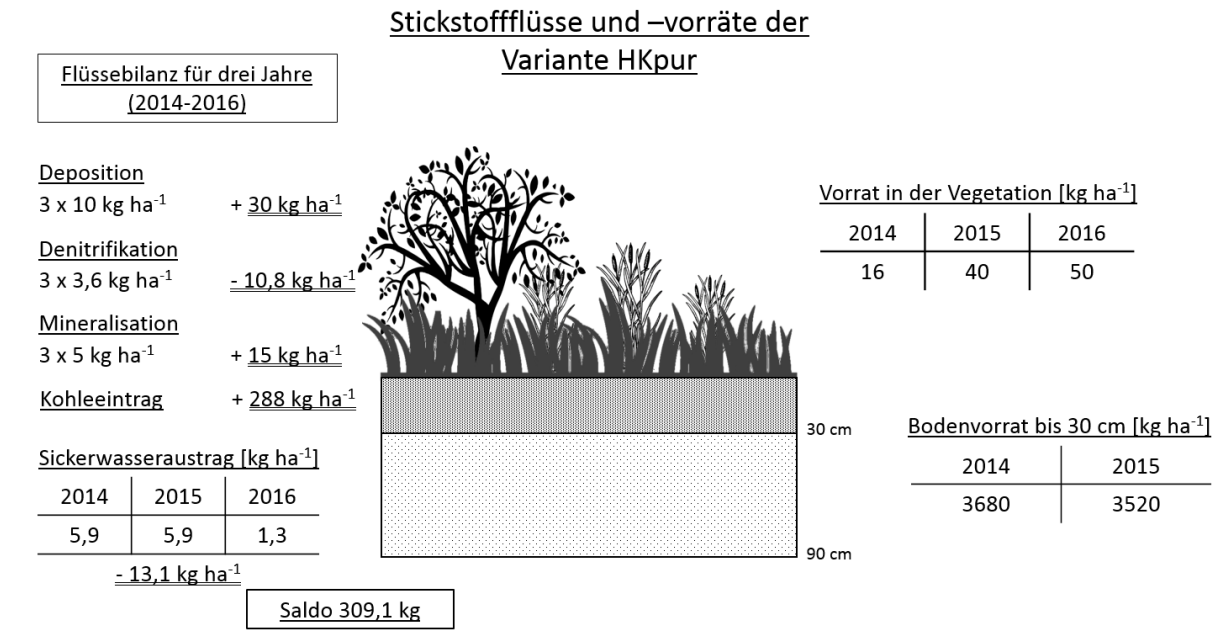

Abbildung 4.3: Stickstoffflüsse und -vorräte der Variante HKpur in den Jahren 2014 - 2016. 


\section{Stickstoffflüsse und -vorräte der} Variante $\mathrm{HK}+\mathrm{N}+\mathrm{Ko}$

ïssebilanz für drei Jahre (2014-2016)

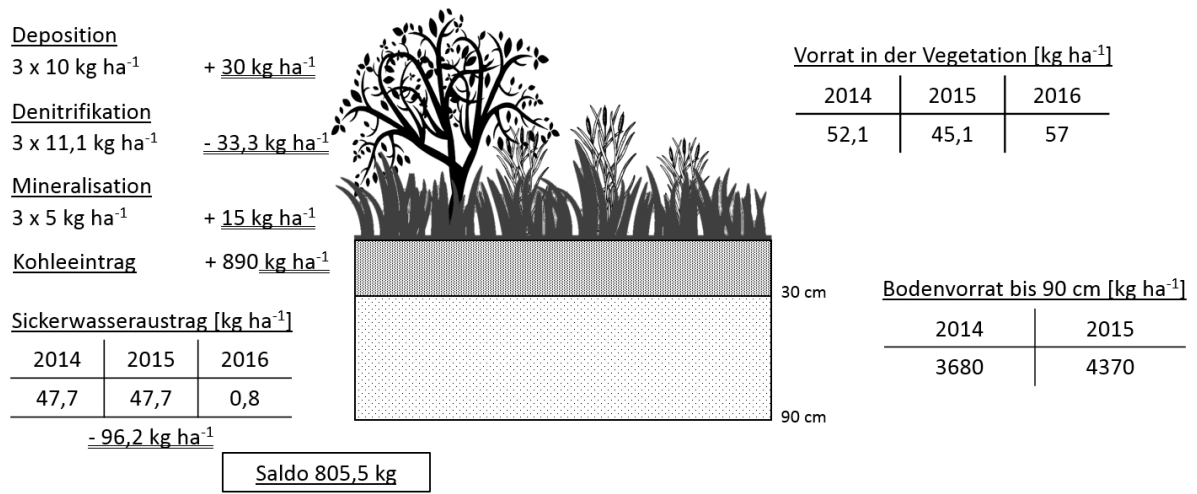

Abbildung 4.4: Stickstoffflüsse und -vorräte der Variante HK+N+Ko in den Jahren 2014 - 2016.

Variante HKpur weist eine weniger gute Übereinstimmung zwischen Saldo und Vorratsdifferenz auf. Der positive Saldo von etwa $300 \mathrm{~kg} \mathrm{ha}^{-1}$ schlägt sich nicht in höheren Stickstoffvorräten im Oberboden nieder. Grund dafür kann, wie bereits erwähnt, die unbeabsichtigte Aussiebung von stickstoffhaltigen Kohlepartikelns im Vorfeld der chemischen Analyse sein.

Die Indikatoren der Stickstoff-Pflanzenernährung und der Stickstoff-Sickerwasserausträge sprechen dafür, dass die Saldomengen des Stickstoffes nicht unmittelbar systemwirksam, sondern relativ stark an den Kohlen fixiert sind. 


\subsection{Wirkungen der Biokohle}

\subsubsection{Begleitvegetation}

Der hohe Anteil stickstoffanzeigender Arten im Jahr 2014 und 2015 auf den Parzellen der stickstoff- $(+\mathrm{N})$ und kompost- $(+\mathrm{N}+\mathrm{Ko})$ behandelten Kohlevarianten deutet eine hohe Stickstoffverfügbarkeit auf diesen Parzellen an.

Zeigerpflanzen reagieren durch ihre Funktion als Bioindikator auf Veränderungen des Standortes. Als ortsfeste Organismen reagieren sie, indem sie schwächeren oder stärkeren Wuchs bzw. Vermehrung aufweisen oder verschwinden. Das stärkere Wachstum der Vegetation ist zunächst als Anpassungsprozess an eine bessere Stickstoffverfügbarkeit bzw. eine vollständige Nutzung des Stickstoffpools zu interpretieren und stellt nicht notwendigerweise einen Indikator für eine Stickstoffsättigung des Systems dar (Eichhorn 1995). Die Veränderung der floristischen Zusammensetzung kann ein Indikator für langfristige Belastungszustände sein (Larcher 1994). So weisen nitrophile Pflanzenarten durch die bessere Stickstoffverwertung meist ein stärkeres Wachstum auf und verdrängen an stickstoffärmere Verhältnisse angepasste Pflanzen (Roloff 1989).

Die aufkommende Begleitvegetation wirkt auf den Parzellen der stickstoff- $(+\mathrm{N})$ und kompost$(+\mathrm{N}+\mathrm{Ko})$ behandelten Kohlevarianten als bedeutender Zwischenspeicher für Stickstoff während der Vegetationsperiode. Durch die nitrophile Artzusammensetzung und die hohen Deckungsgrade sind hier vergleichsweise hohe Stickstoffmengen von etwa $50 \mathrm{~kg} \mathrm{~N}$ ha-1 oberirdisch gebunden. Eichhorn (1995) hat in einem stickstoffgesättigten Buchenökosystem eine oberirdische Stickstoffbindung von $56 \mathrm{~kg} \mathrm{~N}_{\mathrm{Na}}{ }^{-1}$ in der dichten Brennesselvegetation ermittelt.

Die Parzellen der puren Kohlevarianten liegen im Deckungsgrad zwischen der Gruppe der stickstoff$(+\mathrm{N})$ und kompost- $(+\mathrm{N}+\mathrm{Ko})$ behandelten Kohlevarianten und der Nullvariante. Dies bestätigt die Theorie des „Priming-Effektes“ der puren Kohlen.

\subsubsection{Stickstoffaufnahme durch Hainbuche und Kiefer}

Die enormen Mengen des durch die stickstoff- $(+\mathrm{N})$ und kompost- $(+\mathrm{N}+\mathrm{Ko})$ nachbehandelten Kohlevarianten eingebrachten Stickstoffes lassen sich in den Blattstickstoffgehalten nur während der Initialphase (2014/2015) nachvollziehen. Die Stickstoffgehalte und SPAD-Werte der Hainbuchenblätter dieser Versuchsvarianten zeigen für im Jahr 2015 eine Optimal- bis Überversorgung mit Stickstoff und liegen weit über den puren Kohlevarianten und der Nullvariante. Die Ergebnisse des Topfversuches lassen für die Initialphase im Jahr 2014 eine noch höhere Stickstoffverfügbarkeit der stickstoff- $(+\mathrm{N})$ und kompost- $(+\mathrm{N}+\mathrm{Ko})$ nachbehandelten Kohlevarianten annehmen. Die Hainbuchen der puren Kohlen (HKpur bzw. SKpur) weisen im Topfversuch dagegen geringere SPAD-Werte als die Hainbuchen der Nullvariante auf. Dieser Befund bekräftigt eine, im Vergleich zur Nullvariante, reduzierte Stickstoffverfügbarkeit in der Initialphase des Versuches.

Im Feldversuch sinken im Jahr 2016 die Stickstoffgehalte und SPAD-Werte der Hainbuchen in den stickstoff- $(+\mathrm{N})$ und kompost- $(+\mathrm{N}+\mathrm{Ko})$ nachbehandelten Kohlevarianten deutlich ab und liegen unterhalb der Pflanzen der Nullvariante in einem mangelhaften Stickstoffversorgungsbereich. Für die Pflanzen der stickstoff- $(+\mathrm{N})$ und kompost- $(+\mathrm{N}+\mathrm{Ko})$ nachbehandelten Kohlevarianten folgt damit einer Phase mit sehr guter Stickstoffversorgung (2014 und 2015) eine Phase mit deutlich geringerer Stickstoffversorgung.

Im Jahr 2016 weisen die Kiefern der stickstoff- $(+\mathrm{N})$ und kompost- $(+\mathrm{N}+\mathrm{Ko})$ nachbehandelten Kohlevarianten deutlich reduzierte Stickstoffgehalte des letztjährigen (2015er) Nadeljahrganges auf (im Vergleich zum 2016er Nadeljahrgang). Dies kann durch Beobachtungen von Reemtsma (1986) als Hinweis auf einen Versorgungsengpass mit Stickstoff gewertet werden.

Die Einschätzung der Nährstoffverhältnisse durch andere Bewertungsrahmen (Flückiger und 
Braun 2003; Mellert und Göttlein 2012) stützen die Vermutung eines Stickstoffmangels (in Relation zu anderen Nährkationen), da die festgestellten Abweichungen vom harmonischen Elementverhältnis meist unterhalb der empfohlenen Verhältnisbereiche liegen.

Für beide Baumarten lassen sich weitere indirekte Hinweise für eine Stickstoffüberversorgung in der Initialphase des Versuches finden. So weisen beide Baumarten der stickstoff- $(+\mathrm{N})$ und kompost- $(+\mathrm{N}+\mathrm{Ko})$ nachbehandelten Kohlevarianten im Jahr 2015 eine im Vergleich zu den puren Kohlevarianten verringerte Kaliumkonzentration in Blättern und Nadeln auf.

Eine überwiegende Ammoniumernährung der Bäume in diesem Versuch kann aufgrund der Ammoniumpräferenz in der Stickstoffernährung angenommen werden (siehe Kasten in Abschnitt 4.3.5.1). Dies zieht aus Gründen des elektrostatischen Gleichgewichtes eine verstärkte Aufnahme von Anionen wie Phosphat, Sulfat und Chlorid, soweit pflanzenverfügbar, nach sich und behindert so die Aufnahme weiterer konkurrierender Kationen wie Calcium, Magnesium und Kalium $\left(\mathrm{NH}_{4}{ }^{+} / \mathrm{K}^{+}\right.$-Antagonismus) (Riley und Barber 1971; Hock 1988; Kinzel 1982; Boxman und Roelofs 1988; Hoopen et al. 2010). Zudem kann ein erhöhter Kaliumbedarf der Pflanze induziert werden, da Kalium für die Proteinsynthese aus Ammonium benötigt wird. Auch eine Kaliumabgabe von Wurzeln an die Rhizosphäre (Kalium-Leaching) bei überwiegender Ammoniumernährung wird von Hock (1988) beschrieben.

\subsubsection{Andere Makronährstoffe}

Während die Kohlenstoff-, Stickstoff- und Phosphorkonzentration der Variante SKpur vergleichsweise hoch für pure Kohlen aus Getreidespelzen ist, zeigen die übrigen Gehalte an Makronährstoffen typische Werte für pyrolisierte Kohlen (Rajkovich et al. 2012).

Die Variante SKpur weist im Vergleich zur HKpur-Variante einen 6-fach höheren Kaliumgehalt auf, der sich in erhöhten Kaliumgehalten in Blättern und Nadeln niederschlägt.

Die Calcium-, Phosphor- und Kaliumgehalte der Holzkohlenvariante liegen dagegen etwas höher als Rajkovich et al. (2012) und Yao et al. (2012) für Kohlen aus holzigen Ausgangssubstraten ermittelt haben. Steigende Anteile an Rinde, jungen Trieben und Blättern erhöhen die Nährstoffgehalte in Biokohlen (Haubold-Rosar et al. 2014). Angst und Sohi (2013) haben in einem Extraktionsversuch mit unbehandelten Kohlen aus holzigem Ausgangssubstrat eine langanhaltende und gleichbleibende Phosphorfreisetzung und demgegenüber eine nur kurzfristige Freisetzung von Kalium feststellen können. Sie schließen daraus auf eine stabile Phophorversorgung und eine mögliche Kaliumunterversorgung nach einer Kohleeinarbeitung in den Oberboden. Während in dieser Untersuchung die Phosphorgehalte in den Hainbuchenblättern der puren Holzkohlevariante (HKpur) in Topf- und Feldversuch kaum erhöht sind, weisen die Hainbuchenblätter dieser Variante im Vergleich zur Nullvariante höhere Kaliumgehalte auf. Die Langfristigkeit dieser Effekte kann im Rahmen dieser Arbeit nicht abschließend geklärt werden.

Um die Kohlen nach der Pyrolyse abzusäuern und die innere Oberfläche zu vergrößern wurde Phosphorsäure aufgesprüht. Vor allem die stickstoffbehandelten $(+\mathrm{N})$ Kohlevarianten zeigen im Extraktionsversuch deutlich erhöhte Phosphatausträge (Daten nicht präsentiert). Dieser Befund wird durch sehr hohe Phosphorgehalte der Hainbuchenblätter dieser Varianten bestätigt. Die kompostnachbehandelten $(+\mathrm{N}+\mathrm{Ko})$ Kohlevarianten weisen im Vergleich dazu verringerte Phosphatkonzentrationen in den Hainbuchenblättern und im Sickerwasser (Extraktionsversuch) auf.

\subsubsection{Schutz vor Trockenstress}

Eine Übersicht über die in dieser Arbeit beobachteten Effekte von Trockenheit auf Pflanzen gibt Tabelle 4.2. Eine detaillierte Darstellung von gemessenen Indikatoren, Zeiträumen und Varian- 
tenunterschieden findet sich auf Seite 85.

Tabelle 4.2: In dieser Arbeit beobachtete kurz-, mittel- und langfristige Pflanzenreaktionen auf Trockenstress. Die Definition der zeitlichen Skalierung entspricht der Definition der Ergebniszusammenfassung auf Seite 85.

\begin{tabular}{ll}
\hline Zeitskala & Auswirkungen \\
\hline kurzfristig & Stomataverschluss, Blattwassergehalt \\
mittelfristig & frühzeitiger Laubfall, Zurücktrocknen der Zweige, Erweiterung des Wurzel- \\
& systems, Wuchsreduktion (Durchmesser), Mortalität \\
langfristig & $\begin{array}{l}\text { Wuchsreduktion (Durchmesser), Veränderung der Blattmorphologie (Flä- } \\
\text { che, Trockenmasse), Mortalität }\end{array}$ \\
\hline
\end{tabular}

Die an der Ausbildung der Stressresistenz beteiligten Pflanzenprozesse sind meist nicht äußerlich sichtbar. Deshalb kommt den physiologischen Indikatoren, die die innere Stressbelastung in der Pflanze abbilden, eine wichtige Rolle in der Frühindikation von Stresszuständen zu.

Während Trockenperioden in den Jahren 2015 und 2016 wurde im Feldversuch eine deutlich reduzierte stomatäre Leitfähigkeit bei den Hainbuchen in allen Versuchsvarianten festgestellt. An den Messterminen zeigen die Hainbuchen in allen Versuchsvarianten eine Trockenstressbelastung an. Während der späten Vegetationszeit (August) 2016 in einer eher moderaten Trockenperiode zeigen die Hainbuchen der Nullvariante einen stärker reduzierten Wasserdampftransport durch die Stomata, was als Hinweis auf eine höhere Trockenstressbelastung gedeutet werden kann.

Lemoine et al. (2002) haben eine stomatäre Leitfähigkeit von etwa $300-350 \mathrm{mmol} \mathrm{s}^{-1} \mathrm{~m}^{-2}$ in der unbeschatteten Krone einer gut wasserversorgten Buche gemessen. Für verschiedene Laubbaumarten konnten Buschmann et al. (1999) Werte zwischen 170 und $350 \mathrm{mmol} \mathrm{s}^{-1} \mathrm{~m}^{-2}$ für eine ungehemmt ablaufende stomatäre Leitfähigkeit (Maximalwert) ermitteln. Stomatäre Leitfähigkeiten von unter $50 \mathrm{mmol} \mathrm{s} \mathrm{m}^{-2}$ sind als außerordentlich niedrig zu bewerten und nah am Bereich von beginnenden Embolieschäden (Lemoine et al. 2002).

Auch der Blattwassergehalt der Hainbuchen zeigt während der Trockenperioden in den Jahren 2015 und 2016 bei der Nullvariante durchweg geringere Werte als bei den Hainbuchen der Kohlevarianten.

Kursar et al. (2009) haben an tropischen Baumarten gezeigt, dass die Toleranz eines niedrigen Blattwassergehaltes ein Vorteil für das Überleben während Trockenperioden ist. Daher ist ein niedriger Blattwassergehalt nicht zwangsläufig Ausdruck eines höheren Stressniveaus sondern kann auch Ausdruck einer Anpassungsfähigkeit gegenüber Trockenheit sein.

Die Hainbuchen der Nullvariante weisen sehr geringe Kaliumgehalte in den Blättern auf (mangelhafter Versorgungszustand).

Kalium wird für die Stomatakontrolle und die Aufrechterhaltung des Turgors in Nadeln und Blattern benötigt (Fischer und Hsiao 1968; Cochrane und Cochrane 2009). Hohe Kaliumgehalte in den Wurzeln sorgen für ein Gefälle in der Salzkonzentration zum umgebenden Wurzelraum, was die Wasseraufnahme erleichtert. Ein durch überwiegende Ammoniumernährung induzierter Kaliummangel kann somit zu erhöhtem Trockenstress führen (Mengel und Kirkby 1978; Cakmak 2005).

Die Transpiration und das damit ausgelöste Wassersättigungsdefizit in den Zellen ist die treibende Kraft für den Wassertransport vom Boden in die Blätter (Angeles et al. 2004), sie hält 
das Wasserpotenzial in der Pflanze aufrecht (Cruiziat et al. 2002). Während einer Trockenperiode steht weniger pflanzenverfügbares Wasser im Boden zur Verfügung und der ausgelöste Wassermangel stellt für den Organismus eine stressauslösende Belastung dar. Ein hoher Evaporationsbedarf in Verbindung mit niedrigen Bodenwassergehalten führt zu einem Absinken des Wasserpotenzials. Andauernder Wassermangel im Boden verursacht eine Abfolge von Ereignissen des Wassertransports im System Boden-Pflanze-Atmosphäre (Bréda et al. 2006). Auf Kosten einer reduzierten $\mathrm{CO}_{2}$-Assimilation werden die Spaltöffnungen geschlossen und Blätter eingerollt. Das Baumwachstum wird reduziert, es werden kürzere Triebe und kleinere Blätter ausgebildet. Auch die Baumvitalität kann beeinträchtigt werden (Riek und Renger 1994; Bréda et al. 2006), so können durch weiter andauernde Austrocknung Alterungsprozesse, zu beobachten an der Vergilbung von Blättern, beschleunigt werden.

Bei andauernder Trockenheit können durch Abbruch der Wassersäule und Gefäßembolien irreversible Schäden im Xylem-Wassertransport auftreten (Tyree und Ewers 1991; Cruiziat et al. 2002). Ein vorzeitiges Absterben von Wurzelspitzen oder Zweigen kann die Folge sein. Das Abwerfen von Blättern oder Zweigen kann als eine Überlebenstrategie interpretiert werden, um weitere Embolieschäden am Spross zu vermeiden (Tyree et al. 1993). Die Minimierung von Transpirationsverlusten (Stomataverschluss) ist aber der effizientere Weg um mit geringem pflanzenverfügbaren Bodenwasser zurechtzukommen (Cochard et al. 2001; Bréda et al. 2006) und daher Ausdruck eines hohen Resilienzvermögens der Pflanze.

Trotz teilweise höherer Trockenstressbelastung der Hainbuchen der Nullvariante (Kaliumgehalt, stomatäre Leitfähigkeit, Blattwassergehalt) weist die Nullvariante zusammen mit der puren Holzkohlevariante (HKpur) die höchsten Überlebensraten auf. Dies spricht für eine erfolgreiche Anpassung während der Trockenstressphasen.

\subsubsection{Wirkung der Biokohle auf die Vitalität von Hainbuche und Kiefer}

\subsubsection{Mortalität}

Die Hainbuchen zeigen im Feldversuch eine deutlich geringere Mortalität als die Kiefer. Die Hainbuchen der stickstoff- $(+\mathrm{N})$ und kompost- $(+\mathrm{N}+\mathrm{Ko})$ nachbehandelten Kohlevarianten weisen in den Jahren 2014 und 2015 jedoch deutlich höhere Mortalitätszahlen auf, als die Hainbuchen in beiden puren Kohlenvarianten und der Nullvariante. Die Hainbuchen der sticktoff $(+\mathrm{N})$ nachbehandelten Kohlevarianten auch noch im Jahr 2016.

Im Topfversuch zeigen die Hainbuchen einer $\mathrm{HK}+\mathrm{N}$, der $\mathrm{HK}+\mathrm{N}+\mathrm{Ko}$ und der Nullvariante die höchste Überlebensdauer in der Austrocknungsphase. Die Kiefern der puren Kohlenvarianten und der Nullvariante überdauern im Topfversuch eine 5-monatige Austrocknungsphase fast ohne Ausfälle. Damit ist kein positiver Einfluss der Kohlen im Vergleich zur Nullvariante hinsichtlich einer höheren Überlebensdauer feststellbar. Weder im Topf- noch im Feldversuch haben sich höhere Überlebensraten im Vergleich zur Nullvariante gezeigt.

Die Kiefern zeigen in den Jahren 2014 und 2015 bei den stickstoff- $(+\mathrm{N})$ und kompostnachbehandelten $(+\mathrm{N}+\mathrm{Ko})$ Kohlevarianten sehr hohe Mortalitätszahlen. Die kompostnachbehandelte $(+\mathrm{N}+\mathrm{Ko})$ Versuchsvariante weist in beiden Jahren einen Totalausfall auf.

Damit zeigen die Überlebenszahlen im Feldversuch für die Kiefer einen deutlichen Zusammenhang mit der Stickstoffverfügbarkeit. Auch die Kiefern der stickstoffnachbehandelten $(+\mathrm{N})$ Kohlevarianten zeigen während des Topfversuches noch in der Bewässerungsphase erste Absterbeerscheinungen. In den Jahren 2016 und 2017 zeigen die im Jahr 2016 geplanzte Kiefern höhere 
Überlebensraten als in den Vorjahren, ohne Unterschiede zwischen den Versuchsvarianten.

Für eine Reaktion auf die sehr hohe Stickstoffverfügbarkeit sprechen die Variantenunterschiede in der Initialphase 2014 und 2015 im Feldversuch und im Topfversuch, die ab dem Jahr 2016 kaum noch zu beobachten sind. Für den Zeitraum ab 2016 zeigen die Indikatoren StickstoffSickerwasserausträge und -Blattgehalte eine deutlich reduzierte Stickstoffverfügbarkeit im Vergleich zur Nullvariante.

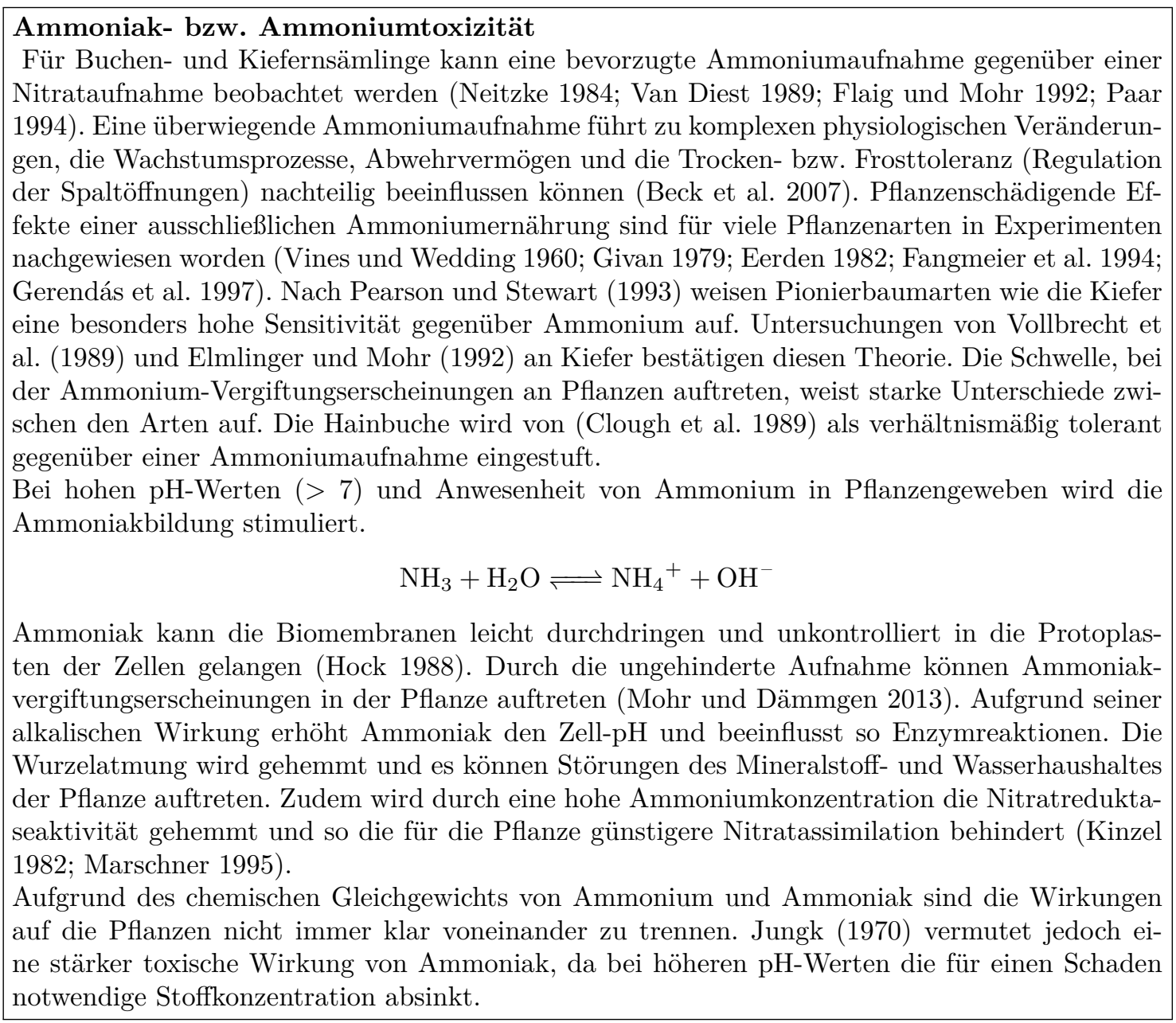

Die genaue stoffliche Ursache für das Absterben von Kiefern und Hainbuchen in der Anfangsphase des Feldversuches kann nicht endgültig geklärt werden. Die Vermutung liegt nahe, dass toxische Konzentrationen von Stickstoffverbindungen wie Ammonium oder Ammoniak eine Rolle spielen (siehe Kasten). Die Ursache der deutlich erhöhten Stickstoffverfügbarkeit liegt in der Stickstoffnachbehandlung der Stickstoff- $(+\mathrm{N})$ und Kompost- $(+\mathrm{N}+\mathrm{Ko})$ Kohlevarianten, die einen Stickstoffeintrag bis zu $1133 \mathrm{~kg} \mathrm{ha}^{-1}$ nach sich gezogen hat.

Die Wuchsbedingungen auf Pionierstandorten sind meist durch eine Stickstofflimitierung gekennzeichnet. Deshalb setzen Pflanzenarten wie die Kiefer, die diese Standorte besiedeln Effizienzstrategien ein, um trotz mäßiger Nährstoffversorgung die volle Stoffwechselleistung zu erreichen und 
konkurrenzfähig zu bleiben (Ernst 1983). Dazu gehören die Erhöhung der Wirksamkeit der Mineralstoffaufnahme durch stärkeres Wurzelwachstum (Absorptionseffizienz) und eine Erhöhung der Nährstoffverfügbarkeit durch die Wurzelausscheidung von Säuren und Chelatbildnern (Mobilisierungseffizienz). Eine ausgeprägte Gebrauchseffizienz der Nährstoffe (Retranslokationseffizienz), die durch Umverteilung in der Pflanze wiederverwendet und damit längerfristig gespeichert werden, ermöglicht das Überdauern in einem nährstoffarmen Lebensraum (Larcher 1994). Auch die Mykorrhizierung von Kiefernwurzeln ist Ausdruck einer Anpassung an stickstoffarme Verhältnisse. So nimmt die Mykorrhizierung von Kiefernfeinwurzeln mit zunehmender Stickstoffversorgung ab (Ritter und Tolle 1978).

Diese Anpassung an nährstoffarme Standorte macht die Kiefer möglicherweise anfälliger gegenüber einer Nährstoffüberversorgung als die Hainbuche, die besser versorgte Standorte besiedelt.

\subsubsection{Wachstum}

Wurzelhalsdurchmesser und Jahrestrieb Die Hainbuchen der Kohlevarianten haben in der trockenen Vegetationsperiode 2015 einen höheren Zuwachs im Wurzelhalsdurchmesser als die Hainbuchen der Nullvariante. Das deutet möglicherweise darauf hin, dass die Pflanzen der Kohlevarianten das Wachstum während der Trockenperiode in geringerem Maße, als die Pflanzen der Nullvariante haben einschränken müssen. Die erhöhte Wasserhaltekapazität des Oberbodens erlaubt den Hainbuchen der Kohlevarianten eine uneingeschränktere Photosynthese und folglich einen höheren Biomasseaufbau.

Bei der Kiefer zeigen sich im Topfversuch deutlich gehemmte Zuwächse im Jahrestrieb der stickstoff- $(+\mathrm{N})$ nachbehandelten Kohlevarianten.

Allgemein folgt die Wuchsreaktion einer Pflanze auf Umweltfaktoren, wie die Stickstoffverfügbarkeit meist einer Optimumkurve (Magill et al. 1997). In diesem Versuch ist bei den stickstoff$(+\mathrm{N})$ und kompost- $(+\mathrm{N}+\mathrm{Ko})$ nachbehandelten Kohlevarianten durch den enormen Stickstoffeintrag eine sehr hohe Stickstoffverfügbarkeit zu beobachten. Es kann davon ausgegangen werden, dass der für das Wachstum der Kiefer optimale Bereich der Stickstoffverfügbarkeit überschritten worden ist. Bei der Interpretation von Wuchsreaktionen muss also berücksichtigt werden auf welchem Abschnitt der Optimumkurve sich das Ökosystem befindet. So haben Kahle et al. (2005), Laubhann et al. (2009) und Solberg et al. (2009) zwar wachstumsteigernde Effekte durch höhere Stickstoffeinträge festgestellt. Die Mehrzahl der beobachteten Ökosysteme zeigt jedoch eine leichte Mangelversorgung mit Stickstoff und befindet sich somit tendenziell im aufsteigenden Kurvenbereich der Optimalkurve (Stefan et al. 1997). Bei jährlichen Stickstoffeinträgen zwischen 50 und $150 \mathrm{~kg} \mathrm{~N}^{-1}$ haben Magill et al. (1997) ein verringertes Wachstum und erhöhte Mortalitätsraten an Pinus resinosa im Harvard Forest beobachtet. Der Effekt der verminderten Biomassenzuwächse nach starker Stickstoffdüngung wird in einigen Untersuchungen mit dem Ammonium/Kationen-Antagonismus als Folge einer überwiegenden Ammoniumernährung (siehe 4.3.2 auf S. 99) in Zusammenhang gebracht (Kinzel 1982; Boxman und Roelofs 1988).

Für die Hainbuche sind in dieser Untersuchung keine Zuwachsänderungen in Abhängigkeit von der Stickstoffverfügbarkeit zu beobachten. 
Biomasseallokation und Wurzelmorphologie Die höheren Feinwurzelanteile an der Gesamtbiomasse und niedrigeren Spross-Wurzelverhältnisse der Hainbuchenpflanzen im Feldversuch des Jahres 2015 im Vergleich zu den Jahren 2016 und 2017 können mögliche Anpassungsreaktionen der Pflanzen auf die Dürre im Jahr 2015 sein.

Eine geringe Bodenfeuchtigkeit bzw. ein leichter Trockenstressreiz der Pflanzen, kann zu stärkerem Feinwurzelwachstum führen (Santantonio und Hermann 1985; Comeau und Kimmins 1989). Auch McMichael und Quisenberry (1993) beobachteten während der Austrocknung eines Bodens eine deutliche Zunahme der Wurzeldichte. Eine verstärkte Biomasseallokation in unterirdische Pflanzenkompartimente trägt zwar zu einer höheren Stressresistenz gegenüber Trockenheit bei (Bréda et al. 2006), bedeutet aber eine zusätzliche Ressourceninvestition.

Der Anteil der Feinwurzeltrockenmasse an der Gesamttrockenmasse kann als Indikator des Wasseraufnahmepotentials interpretiert werden. Die Sicherung der Wasseraufnahme durch erhöhtes Wurzelwachstum, verbesserten Wassertransport und eine Wasserspeicherung in den Pflanzengeweben gehört zu den Strategien der Stressresistenz (Saxe et al. 1998).

Die Spross-Wurzel-Verhältnisse der Hainbuchen im Feldversuch weisen nach drei Jahren bei der puren Holzkohlevariante (HKpur) und der Nullvariante geringere Werte auf. Die Kiefer zeigt dieses Muster nicht. An den Hainbuchen und Kiefern der Variante HKpur lassen sich höhere Feinwurzelanteile beobachten.

Nach der Theorie der funktionellen Biomasseallokation (Enquist und Niklas 2002; Poorter et al. 2012) kann die Biomasseallokation als eine morphologische Anpassungsreaktion der Pflanze auf eine Mangelversorgung mit Wasser und/oder Nährstoffen interpretiert werden. Die Stickstoffverfügbarkeit hat einen großen Einfluss auf die Aufteilung in ober- und unterirdische Biomasse (Cambui et al. 2011). Eine bessere Stickstoffversorgung kann beispielsweise zu geringeren Feinwurzelbiomassen (Rapp 1991; Vogt et al. 1986; Eichhorn 1995) führen. Für Douglasie haben Keyes und Grier (1981) und Vogt et al. (1983) auf nährstoffarmen Standorten deutlich höhere Anteile der Feinwurzelbiomasse an der Gesamtbiomasse ermittelt, als auf gut nährstoffversorgten Standorten.

Je schlechter die Nährstoffversorgung, umso stärker kann das Wachstum durch eine Nährstoffgabe angeregt werden. Diese Beobachtung wird durch Zöttl (1964) für das Wachstum von Feinwurzeln in Fichtenbeständen bestätigt. Auf nährstoffarmen Moorstandorten wurde durch Paavilainen (1967) eine deutliche Erhöhung der Feinwurzelbiomasse von Kiefernbeständen durch NPK-Düngung beobachtet. Vogt et al. (1985) haben dagegen für Douglasie eine abnehmende Feinwurzelbiomasse nach NPK-Düngung festgestellt.

Paar (1994) hat in Nährlösungsexperimenten mit Buche den Zusammenhang zwischen steigender Ammoniumaufnahme und einer Abnahme von Feinwurzeln beschrieben. Mögliche Erklärungsansätze sind vielfältig, so werden eine lokale Bodenversauerung oder ein rascher Kohlenhydratverbrauch durch die Ammonium-Aufnahme diskutiert. Ein durch die Ammoniumaufnahme induzierter Kaliummangel wird von Flaig und Mohr (1992) für geringere Wurzeltrockenmassen an Kiefernkeimlingen bei reiner Ammoniumernährung verantwortlich gemacht. Rost-Siebert (1985) berichtet bei reiner Ammoniumernährung von stärkeren Wurzelschäden an Buchenkeimlingen, als bei reiner Nitrat- oder gemischter Ammonium-Nitraternährung.

Aber auch eine hohe Nitratverfügbarkeit kann zu einer veränderten Allokation von Biomasse führen. So wurde in einigen Studien eine Reduktion der Feinwurzelbiomasse durch hohe Nitrifikationsraten in Laubwäldern beobachtet (Aber et al. 1985; Ibrom und Runge 1989; Rapp 1991). Die höhere Mobilität des Nitrations und eine daraus abgeleitete, für eine effektive Stickstoffaufnahme, reduzierte notwendige Wurzelbiomasse wird von Aber et al. (1985) diskutiert. 
In dieser Untersuchung ist im Jahr 2015, also nach einer Dürrephase und während einer Phase zum Teil sehr hoher Stickstoffverfügbarkeit, ein Zusammenhang zwischen einer höheren Stickstoffversorgung und abnehmenden Feinwurzelbiomassen bei der Hainbuche zu beobachten. In den Folgejahren liegt die Stickstoffverfügbarkeit besonders auf den Parzellen der stickstoff- $(+\mathrm{N})$ und kompostnachbehandelten $(+\mathrm{N}+\mathrm{Ko})$ Kohlevarianten sehr niedrig und unterhalb der Nullvariante. Die höheren Feinwurzelanteile der Hainbuche in den Jahren 2016 und 2017 zeigen damit ebenfalls den Zusammenhang mit der aktuellen Stickstoffverfügbarkeit. Für die Kiefer kann im Jahr 2016 ein schwacher Zusammenhang zwischen der aktuellen Stickstoffversorgung und der gemessenen Feinwurzelbiomassenanteile beobachtet werden.

Die im Vergleich zu den Hainbuchen geringeren Feinwurzelmassen der Kiefern stehen im Einklang mit Untersuchungen von Finér et al. (2007), die die Baumarten Buche, Fichte und Kiefer näher untersucht haben. Danach verfügen Laubbäume über höhere Feinwurzelmassen als Nadelbäume. Mit zunehmendem Alter weist die Buche abnehmende, die Kiefer steigende Feinwurzelmassen auf (Finér et al. 2007). Die bessere Anpassung der Kiefer an Trockenheit ist im Jugendstadium eher auf effizientere Schutzmechanismen gegen Wasserverluste zurückzuführen (Kätzel et al. 2015), als auf ein besser entwickeltes Wurzelsystem.

In dieser Arbeit zeigen die Hainbuchen der Nullvariante im Feldversuch 2016 einen deutlich höheren Anteil trockener Wurzelspitzen als die übrigen Kohlevarianten.

Die höheren Anteile trockener Wurzelspitzen der Nullvariante im Jahr 2016 kann mit dem Befund der höheren Feinwurzelbiomasse dieser Variante im Jahr 2015 in Zusammenhang gebracht werden. Eine höhere Feinwurzelbiomasse bedingt eine höhere Umsetzungsrate (Turn-Over). Sie sind demnach Resultat einer Anpassungsreaktion an eine Trockenstressbelastung im Sommer 2015. Bréda et al. (2006) beobachteten höhere Absterbeerscheinungen der Feinwurzeln während Trockenperioden.

Mykorrhizierung In dieser Untersuchung ist im Sommer 2016 also etwa 2,5 Jahre nach Versuchsanlage und einem damit verbundenen Stickstoffeintrag zwischen 290 und $1130 \mathrm{~kg}^{-1}$ bei der Hainbuche kein negativer Einfluss der Kohlen auf die Mykorrhizierung feststellbar. Die Versuchsvarianten mit den höchsten Stickstoffeinträgen $(+\mathrm{N}$ und $+\mathrm{N}+\mathrm{Ko})$ zeigen im Jahr 2016 sogar leicht höhere Mykorrhizierungsgrade als die Nullvariante. Die geringen Stickstoffgehalte der Bodenlösung dieser Varianten zeigen eine hohe Stickstoff-Immobilisierung durch die Kohlen ab dem Jahr 2016. Die Reihenfolge absteigender Mykorrhizierung stellt sich folgendermaßen dar: $+\mathrm{N}+\mathrm{Ko}>+\mathrm{N}>$ pure Kohlen und Null. Allgemein liegen die Mykorrhizierungsgrade bei der Hainbuche zwischen 70 und 95 \%. Die Mykorrhizierung an den Kiefernwurzeln zeigt 2016 nach einer Vegetationszeit keinen Zusammenhang mit der eingetragenen Stickstoffmenge.

Mykorrhizapilze sind in der Lage komplexe organische Verbindungen aufzuschließen (Pena et al. 2013) und Pflanzen mit mineralischen Nährstoffen und Wasser (Wild 1995) zu versorgen. Somit kann die Nährstoffversorgung und die Trockenresistenz von mykorrhizierten Pflanzen erhöht werden (Pena und Polle 2014). Bei Douglasien- und Eichenkeimlingen mit mykorrhizierten Feinwurzeln ist eine verbesserte Wasserversorgung während Trockenphasen beobachtet worden (Dixon et al. 1980; Parke et al. 1983; Garbaye und Churin 1997). Gründe sind eine höhere Absorptionsoberfläche durch die feinen Hyphen, die einen größeren Anteil des in den Feinporen gebundenen Wassers erschließen können. Weitere positiv wirksame Faktoren einer Mykorrhizierung sind eine effizientere Wasserleitung und eine vergrößerte hydraulische Leitfähigkeit an der Boden-Wurzel-Schnittstelle (Boyd et al. 1986; Guehl et al. 1992).

Im Zusammenhang mit den „neuartigen Waldschäden“ konnte eine Schädigung der Mykorrhiza durch hohe Stickstoffeinträge beobachten werden (Meyer 1983; Meyer 1985; Meyer 1988). 
Wöllecke et al. (1999) haben in Nordostdeutschland an einem stark durch Stickstoffdeposition beeinflussten Kiefernbestand eine um $27 \%$ geringere Mykorrhizierung der Wurzelspitzen festgestellt. In einer neueren Untersuchungen stellten De Witte et al. (2017), bedingt durch erhöhte atmosphärische Stickstoffeinträge, eine Abnahme der Häufigkeit mykorrhizierter Wurzelspitzen an Buchen fest. Auch Kjøller et al. (2012) beschreiben negative Effekte auf die Mykorrhiza durch steigende Stickstoffdepositionen. Rapp (1991) hat eine abnehmende relative Mykorrhizahäufigkeit an Wurzeln von Altbuchen nach einer Ammoniumsulfatdüngung festgestellt. Göbl und Platzer (1967) beobachteten einen verringerten Mykorrhizabesatz an Zirben-Jungpflanzen nach Zugabe von Ammoniumsulfatdünger.

Mykorrhizapilze können grundsätzlich von einer Biokohlebeigabe in den Boden profitieren, dies wurde für landwirtschaftliche Kulturen nachgewiesen (Graber et al. 2010). Die inneren Poren der Biokohle fungieren dabei als geeigneter Ort für den Austausch von Nährstoffen und Metaboliten zwischen Substrat und Pflanzenwurzeln (Warnock et al. 2010). In dieser Untersuchung ist dieser Effekt allerdings nicht nachgewiesen worden.

Die etwas höheren Mykorrhizierungsgrade der Hainbuchen der stickstoff- $(+\mathrm{N})$ und kompost$(+\mathrm{N}+\mathrm{Ko})$ nachbehandelten Kohlevarianten können als weiterer Hinweis auf die Immobilisierung (und damit Nicht-Verfügbarkeit) des Stickstoffs durch die Biokohlen zum Zeitpunkt der Probenahme gewertet werden. Dies könnte sich zukünftig als Vorteil für das Überleben während Trockenperioden erweisen. Aufgrund der geringen Unterschiede zwischen den Varianten in der ermittelten Mykorrhizierung, wirken während der versuchsdauer andere Faktoren wie etwa das Spross-Wurzel-Verhältnis stärker auf die Fähigkeit, Trockenperioden zu überstehen.

\subsubsection{Blattmorphologie und Blatt-/Nadelzustand}

Der 2014 und 2015 im Feldversuch beobachtete Blattabwurf der Hainbuche während der Trockenperioden wurde von einem Wiederaustrieb der Blätter an einigen Pflanzen nach Ende der Trockenperiode begleitet.

Der stressbedingte Blattverlust und der folgende Wiederaustrieb bedeutet im Sinne der Allokation von Biomasse eine Senke, da erneut Ressourcen investiert werden müssen um Assimilationsorgane aufzubauen. Das Wiederaustreiben von frischen Blättern nach der Trockenperiode wurde auch von Roloff (2001) beschrieben, der der Hainbuche ein besonderes Vermögen in Trockenperioden grüne Blätter abzuwerfen und bei besseren Wuchsbedingungen wieder auszutreiben, zuschreibt.

Die Hainbuchen haben 2016 im Feldversuch nach der außergewöhnlich trockenen Vegetationsperiode 2015 deutlich kleinere Blätter ausgebildet. Am stärksten ist dieser Effekt bei den Hainbuchen der puren Kohlevarianten und der Nullvariante. Die Hainbuchen der stickstoff- $(+\mathrm{N})$ und kompost- $(+\mathrm{N}+\mathrm{Ko})$ nachbehandelten Kohlevarianten bilden im Jahr 2016 größere und tendenziell leichtere Blätter als Hainbuchen der Null- und puren Kohlevarianten aus.

Das Einrollen der Blätter, der Blattabwurf oder die Ausbildung von kleineren Blattorganen ist eine Anpassungsreaktion der Pflanze gegen unkontrollierbare Wasserverluste und dient einer Verkleinerung der transpirierenden Oberflächen (Larcher 1994). Modifikative Anpassungen der Blätter, wie die Ausbildung von kleineren Blättern mit kleineren Spaltapparaten in größerer Dichte auf der Blattoberfläche (Xeromorphose) haben Battaglia et al. (1998) und Le Dantec et al. (2000) als Reaktion auf wiederholten Trockenstress beobachten können. Dies deckt sich mit Bebachtungen in der Waldzustandserhebung von kleineren Blättern nach einem Trockenjahr an Buche (mdl. Mitteilung, J. Eichhorn). Die Blattfläche ist vor allem Ausdruck der klimatischen Umweltbedingungen des Vorjahres, da die Knospen mit den Blattanlagen bereits im Vorjahr gebildet werden. Dürreperioden im ersten Jahr können zu reduzierten Blattanlagen führen und 
haben damit einen großen Einfluss auf die Blattfläche (Bréda et al. 2006).

Die höheren Überlebenszahlen der Hainbuchen der puren Kohlevarianten und der Nullvariante bestätigt die Wirksamkeit dieser modifikativen Anpassungsreaktionen.

Der Blatt-/Nadelzustand der Hainbuchen und Kiefern zeigt 2015 einen deutlichen und 2016 immer noch einen schwachen Zusammenhang mit der Stickstoffverfügbarkeit. Es können stärkere Blatt- und Nadelverluste an den Pflanzen der stickstoff- $(+\mathrm{N})$ und kompostnachbehandelten $(+\mathrm{N}+\mathrm{Ko})$ Kohlevarianten beobachtet werden. Dies wird von Ergebnissen von Flaig und Mohr (1992) bekräftigt, die bei einer reinen Ammoniumernährung an Kiefernkeimlingen Schäden an sämtlichen Kompartimenten und braune Nadelspitzen feststellen konnten.

\subsubsection{Wechselwirkungen von Stickstoffverfügbarkeit und Trockenheit}

Die Analyse der Überlebenszahlen gibt Hinweise auf eine Wechselwirkung der stickstoff- und trockenstressauslösenden Umweltbedingungen, vor allem in der Anfangsphase des Feldversuches (2014 und 2015) (siehe Abb. 4.7 auf S. 114). Der Stickstoffeintrag hat auf den Parzellen der stickstoff- $(+\mathrm{N})$ und kompost- $(+\mathrm{N}+\mathrm{Ko})$ behandelten Kohlevarianten zu reduzierten WurzelSpross-Verhältnissen der Pflanzen geführt. Diese Anpassungsreaktionen sind in dieser Arbeit für die Hainbuche deutlicher als für die Kiefer nachgewiesen worden. Die hohe Mortalität der Kiefer noch während der ersten Vegetationszeit spricht für ein Überschreiten des Anpassungsvermögens in dieser Phase.

Flückiger und Braun (1998) haben die einseitige Förderung der oberirdischen Sprossorgane zu Lasten des Wachstums der für die Wasser- und Nährstoffaufnahme zuständigen Wurzeln durch erhöhte Stickstoffdepositionen festgestellt. Eine Verschiebung der Biomasseallokation hin zu oberirdischen Pflanzenkompartimenten mit höheren Blattbiomassen haben auch McNulty und Boggs (2010) beobachten können. Aus dem damit verbundenen höheren Wasserbedarf bei geringerer relativer Wurzelbiomasse, die die Wasserversorgung sicherstellt, leiten sie eine höhere Anfälligkeit gegenüber Trockenstressbelastungen ab. Im Gegensatz dazu, haben die Bäume auf nährstoffärmeren Standorten ein günstigeres Wurzel-Sprossverhältnis und damit bessere Voraussetzungen für ein Überleben während Trockenperioden.

Magill et al. (2004) haben nach einer Trockenperiode eine höhere Mortalität und vermindertes Wachstum an einem wiederholt stickstoffgedüngten Kiefern-Mischbestand feststellen können. Bei der Weihrauch-Kiefer haben Pharis und Kramer (1964) bei einer Stickstoffverfügbarkeit, die über dem Optimum liegt, eine reduzierte Trockenresistenz beobachten können.

Nach einer außergewöhnlich trockenen Vegetationsperiode 2015 werden im Folgejahr durch die Hainbuche kleinere Blätter mit höherer Trockenmasse ausgebildet. Die modifikative Verkleinerung der Blattfächen nach einer Dürreperiode ist bei den Hainbuchen der kompostnachbehandelten $(+\mathrm{N}+\mathrm{Ko})$ Kohlevarianten weniger stark ausgeprägt, möglicherweise durch eine gleichzeitig wirkende Tendenz zur stickstoffinduzierten Blattflächenvergrößerung.

Die Anpassung der Blattgröße und -fläche kann somit Ausdruck der Stickstoffverfügbarkeit und der Trockenstressbelastung sein. Eine erhöhte Stickstoffverfügbarkeit kann zu größeren Blattflächen führen, während eine vorjährige Trockenstressbelastung kleinere Blattflächen induzieren kann (McNulty et al. 2014). Die Anpassungsreaktion an Trockenstress verläuft für die Hainbuchen der kompostnachbehandelten $(+\mathrm{N}+\mathrm{Ko})$ Kohlevarianten damit abgeschwächt und geht mit einer höheren Mortalitätswahrscheinlichkeit einher.

Für eine genauere Bewertung dieser Anpassungsreaktion ist die Versuchsdauer jedoch zu kurz. 


\subsection{Zusammenfassende Betrachtungen zur Resilienz von Hainbuche und Kiefer}

Als Resilienz wird die Fähigkeit eines Ökosystems bezeichnet, Störungen zu kompensieren und seine Basisfunktionen weiterhin zu erfüllen, ohne seine grundlegende Organisationsstruktur zu ändern (Holling 1973; Walker und Salt 2012). Anhand der Resilienztheorie kann die Systemstabilität des in dieser Arbeit untersuchten Ökosystems bewertet werden. Dazu bietet sich das „Ball-in-der-Tasse“-Modell nach Walker und Salt (2012) an (siehe Abb. 4.6 auf Seite 111).

Als ein wichtiges Ziel des vorliegenden Versuches kann die Erweiterung der Resilienz gegenüber Störungen wie Dürren oder Stoffeinträgen formuliert werden, um Risiken in der Bewirtschaftung zu minimieren. Die Einarbeitung der Biokohle in den Oberboden ist mit der Absicht geschehen, die Wasserhaltekapazität des Oberbodens zu erhöhen und die Nährstoffversorgung der Jungbäume zu stabilisieren. Die dadurch verbesseten Anwuchsbedingungen sollen die Absterberaten der Forstkultur vor allem in Trockenperioden deutlich senken und so eine höhere Zahl von Optionen hinsichtlich der Flächenbewirtschaftung und der Erfüllung von ökosystemaren Funktionen ermöglichen. Die Nullvariante dient als Referenz, obwohl auch hier die Bodenbearbeitung als versuchsbedingte Störung einwirkt.

\subsubsection{Störungen}

Störungen bzw. Änderungen der Umweltbedingungen sind feste Bestandteile von Waldökosystemen und für deren Dynamik von großer Bedeutung (Otto 1994).

Die in diesem Versuch auftretenden klimatischen Störungen sind Trockenheit und möglicherweise auch Hitze. Das Jahr 2015 weist im Untersuchungsgebiet eine sehr trockene Vegetationsperiode auf, die bis in den Spätsommer anhält. Von April bis Juli erreicht das Niederschlagsdefizit im Rhein-Main-Gebiet mehr als $50 \%$.

Auch der aktuelle Füllstand der nutzbaren Feldkapazität (nFK) kann als Schlüsselindikator einer Störung durch Trockenheit aufgefasst werden. Der modellierte Füllstand der nutzbaren Feldkapazität beträgt im Juni 2015 auf dem nächstgelegenen BZE II-Punkt weniger als 20 \% (Sutmöller et al. 2017). Der von Granier et al. (1999) und Anders et al. (2002) beschriebene Schwellenwert von etwa $40 \%$ der nFK ist damit unterschritten.

Durch die Versuchsanlage bedingt, kommen weitere Störgrößen hinzu. Die bodenvorbereitenden Arbeitsschritte Mulchen und Fräsen sind in allen Versuchsvarianten in gleicher Weise ausgeführt worden. Die intensive Bodenbearbeitung hat Auswirkungen auf Parameter der Bodenphysik (Trockenrohdichte) und der Bodenchemie (Mineralisierungsraten). So ist der Prozess der Bodensetzung nach zwei Jahren noch nicht vollständig abgeschlossen. Eine Störungswirkung durch schlechte Verankerung der Pflanzen im Boden und unvollständigen Boden-Wurzelkontakt kann in der ersten Vegetationsperiode nicht ausgeschlossen werden.

Die unmittelbar vor der Pflanzung in den Oberboden eingebrachte Kohle hat sich zum Pflanzzeitpunkt nicht mit Wasser aufsaugen können. Somit stand die theoretisch durch die Kohle verfügbare nutzbare Feldkapazität nicht von Beginn an zur Verfügung. Es kann in dieser initialen Phase sogar von einer Konkurrenzsituation zwischen Kohle und Pflanzen um das vefügbare Wasser ausgegangen werden. Eine durch die Versuchsanlage induzierte, angespannte Wasserverfügbarkeit bei den Kohlevarianten ist somit nicht auszuschließen.

Die entscheidende versuchsbedingte Störung besteht jedoch in der Nachbehandlung von einigen Kohlesubstraten mit Stickstoff $(+\mathrm{N})$ und Kompost $(+\mathrm{N}+\mathrm{Ko})$, die die Stickstoffverfügbarkeit 
enorm verändert. Indikatoren wie Sickerwasserausträge und Blattgehalte von Stickstoff zeigen die Intensität und die zeitliche Dynamik dieser Störung. Auch während der Vegetationszeit 2015 sind noch Stickstoffausträge zu beobachten. Die Stickstoffaufnahme durch die Vegetation genügt nicht um den Stickstoff im System zu halten. Ein regelmäßiger Stickstoffaustrag kann einen Kipppunkt darstellen, bei dem das System Wald in einen anderen Zustand übergeht (Eichhorn et al. 2016). Nach einigen Autoren ist eine Stickstoffsättigung erreicht bzw. ist ein Schwellenwert der Stickstoffversorgung überschritten, wenn der Eintrag aus Stickstoffmineralisierung und Atmosphäre die Rückhaltefähigkeit des Systems langfristig übersteigt und regelmäßig nennenswerte Stickstoffmengen gasförmig oder über das Sickerwasser aus dem System ausgetragen werden (Aber et al. 1989; Cole et al. 1992; Van Miegroet et al. 1992). Die Stickstoffverfügbarkeit eignet sich in dieser Betrachtung somit als Schlüsselindikator, um Veränderungen des ökosystemaren Zustands in einer Belastungssituation zu erfassen und anschließend zu bewerten. Die Stickstoffkonzentrationen im Sickerwasser des Topf- und Feldversuches zeigen, dass während der ersten beiden Vegetationsperioden (2014 und 2015) bei den stickstoff- $(+\mathrm{N})$ und kompost- $(+\mathrm{N}+\mathrm{Ko})$ nachbehandelten Kohlevarianten nenneswerte Stickstoffmengen mit dem Sickerwasser ausgetragen werden. Die Stickstoffausträge liegen in diesem Zeitraum zwischen 25 und $47 \mathrm{~kg} \mathrm{ha}^{-1}$. In den Folgejahren geht der Stickstoffaustrag, bedingt durch das steigende Immobilisierungspotenzial der Kohlen, deutlich zurück und liegt im Jahr 2017 unter der Nullvariante (außer Variante $\mathrm{HK}+\mathrm{N})$.

Darüber hinaus kann eine Schwellenwertdefinition anhand des Stickstoffeintrages vorgenommen werden. In diesem Versuch lag der Stickstoffeintrag je nach Versuchsvariante zwischen 290 und $1133 \mathrm{~kg} \mathrm{~N} \mathrm{ha}{ }^{-1}$. Das „critical load“ Konzept definiert den Schwellenwert, als den maximalen Eintrag in ein System, der ohne chemische Veränderungen und langfristig ohne nachteilige Auswirkungen erfolgt (Nilsson 1988; Grennfelt und Thörnelöf 1992). Nilsson (1988) geben für die meisten Waldökosysteme einen Schwellenwertbereich zwischen 10 und $20 \mathrm{~kg} \mathrm{~N} \mathrm{ha}^{-1} \mathrm{a}^{-1}$ an. In produktiven mitteleuropäischen Waldökosystemen kann der critical load auch bei $30-40 \mathrm{~kg} \mathrm{~N}$ $\mathrm{ha}^{-1} \mathrm{a}^{-1}$ liegen. Für das in dieser Arbeit betrachtete Waldökosystem werden die kritischen Einträge, wie bei den meisten Waldökosystemen, vermutlich im Bereich von $10-15 \mathrm{~kg} \mathrm{~N} \mathrm{ha}^{-1} \mathrm{a}^{-1}$ liegen (Bobbink und Roelofs 1995; Bobbink et al. 2003) und damit etwas über der aktuellen Stickstoffdeposition von $10 \mathrm{~kg} \mathrm{ha}^{-1}$ (Schaap et al. 2015). Die kohlebedingten Stickstoffeinträge betragen ein Vielfaches dieses critical-loads und führen in den ersten beiden Versuchsjahren zu negativen Auswirkungen bzw. schlechteren Anwuchsbedingungen. Dazu zählen Stickstoffausträge mit dem Sickerwasser, Ernährungsungleichgewichte, veränderte Spross-Wurzel-Verhältnisse und veränderte Begleitvegetation. Durch das hohe Immobilisierungspotenzial der Kohlen können weitere, negative Auswirkungen ab 2016 verhindert werden. Aufgrund der dadurch limitierten Einwirkzeit der kritischen Einträge und der guten Nährstoffausstattung des Versuchsstandortes ist eine mittelfristige Verschlechterung der Standortsqualität nicht zu erwarten. Wie sich der Standort aufgrund der erhöhten Stickstoffvorräte, der weiter wirksamen Immobilisierung und anhaltend hohen Stickstoffdepositionen langfristig verhält, kann nicht abgeschätzt werden. Dazu bedarf es langfristiger Untersuchungen.

Die Störung durch die erhöhte Stickstoffverfügbarkeit der stickstoff- $(+\mathrm{N})$ und kompost- $(+\mathrm{N}+\mathrm{Ko})$ Versuchsvarianten wirkt 2014 und 2015 als systemsteuernde Größe, während die Störung durch Trockenheit vor allem während der Versuchsanlage Anfang 2014, im Sommer 2015 und Anfang 2017 auftritt. 


\subsubsection{Wirkungen der Störungen auf die Resilienz}

Die im Feld- und Topfversuch zu beobachtende, zum Teil sehr hohe Mortalität der Forstpflanzen macht deutlich, dass Schwellenwerte der durch das System kompensierbaren Störungen überschritten worden sind. Die Kombination der Störungen ist hochdynamisch, daher verläuft die Systemantwort (hier: Pflanzenmortalität) nicht immer linear. Sie wird nicht durch die mittleren Bedingungen, sondern von den Extremen bestimmt.

Die nach vier Vegetationszeiten gegenüber der Nullvariante leicht höheren Überlebenszahlen der puren Kohlevariante (HKpur) scheinen zunächst für eine gelungene Erhöhung der Resilienz in dieser Versuchsvariante zu sprechen. Für beide Varianten (HKpur, Null) zeigt sich, dass Störungen besser absorbiert werden und die Basisfunktionen des Ökosystems weitgehend erhalten bleiben. Im Gegensatz dazu, ist durch die Stickstoff- $(+\mathrm{N})$ und Kompost- $(+\mathrm{N}+\mathrm{Ko})$ Nachbehandlung der Kohlen eine deutliche Resilienzminderung eingetreten. Es konnte nachgewiesen werden, dass in diesem Versuch die Stickstoffverfügbarkeit die kontrollierende Variable der Mortalität ist.

In dieser Arbeit findet (mit Ausnahme der physiologischen Indikatoren) eine nachträgliche Betrachtung der für einen Systemübergang (Baummortaliät) relevanten Grenz- und Schwellenwerte statt. Dafür müssen die zu diesen Zeitpunkten wirkenden Störungen (und Störungskombinationen) mit den Absterbeereignissen in Verbindung gebracht werden. Dies stellt aufgrund der zeitlichen Verzögerung zwischen Ursache und Wirkung eine komplexe Aufgabe dar. So können Belastungen durch Trockenperioden neben direkt hervorgerufenen, sicht- oder messbaren Schäden bzw. Anpassungsreaktionen auch verzögert auftretende, längerfristige Auswirkungen zeigen. In diesem Versuch konnten direkte Reaktionen wie die Mortalität, der Blattverlust, das Zurücktrocknen von ober- und unterirdischen Kompartimenten und der Stomataverschluss beobachtet werden. Langfristige Reaktionen waren zum Beispiel die Ausbildung kleinerer Blätter, die Erweiterung des Wurzelsystems und der Wiederaustrieb von Blättern nach einer Trockenperiode bzw. der Stockausschlag im nächsten Jahr. Die langfristigen Anpassungsreaktionen wirken sich resistenzfördernd für die Einzelpflanzen aus und erhöhen somit die Resilienz des Gesamtsystems. Dürreereignisse rufen physiologische Veränderungen in der Kohlenstoff- und Nährstoffassimilation hervor. Geschädigte Pflanzengewebe müssen zunächst mit zusätzlichem Energieaufwand repariert werden, die Kohlenhydratspeicher können somit bis zum Ende der Vegetationsperiode nicht vollständig aufgefüllt werden. Die bestehenden Ressourcen müssen aber weiterhin auf Reparaturen und die Aufrechterhaltung der Lebensfunktionen verteilt werden. Daher können Einschränkungen im Zuwachs und in der Abwehr von sekundären Stressereignissen noch mehrere Jahre nach einer starken Trockenstressbelastung auftreten (Battaglia et al. 1998; Le Dantec et al. 2000; Desprez-Loustau et al. 2006; Rouault et al. 2006).

In Abbildung 4.5 ist für die Versuchsvarianten Null und N+Ko die zeitliche Dynamik der Störungen (Bodenbearbeitung, Stickstoffverfügbarkeit, Trockenheit) ausgewählten Indikatoren der Pflanzenreaktion (Wirkung) gegenübergestellt. Die Störgröße Stickstoffverfügbarkeit besitzt in der Variante N+Ko eine hohe zeitliche Dynamik, die durch eine Abgrenzung von Phasen näher charakterisiert werden kann. So wirken die Störungen Bodenbearbeitung und Stickstoffverfügbarkeit in den ersten beiden Versuchsjahren sehr stark (Initialphase). In diesem Zeitraum (2014/2015) sind die Zahlen der Mortalitäten für beide Baumarten am höchsten. Vitalitätsbezogene Indikatoren, wie Blattverlust und Nadelverfärbung zeigen ebenfalls schlechtere Werte als in der folgenden Phase. Damit stimmt hier der Zeitraum der Stickstoffüberversorgung mit dem Zeitraum störungsspezifischer Wirkungen überein. Im Jahr 2016 liegt die Stickstoffverfügbarkeit auf dem Niveau der Nullvariante, während sie im Folgejahr 2017 meist unter der Nullvariante liegt (Stabilisierungsphase). Die Mortalitätszahlen zeigen in dieser Stabilisierungsphase für bei- 
de Baumarten deutlich geringere Werte als in der vorhergehenden Phase. Auch Indikatoren der Vitalität zeigen bessere Werte. Es besteht somit eine hohe, baumartenspezifische Abhängigkeit der Vitalität und Mortalität gegenüber der Stickstoffverfügbarkeit.

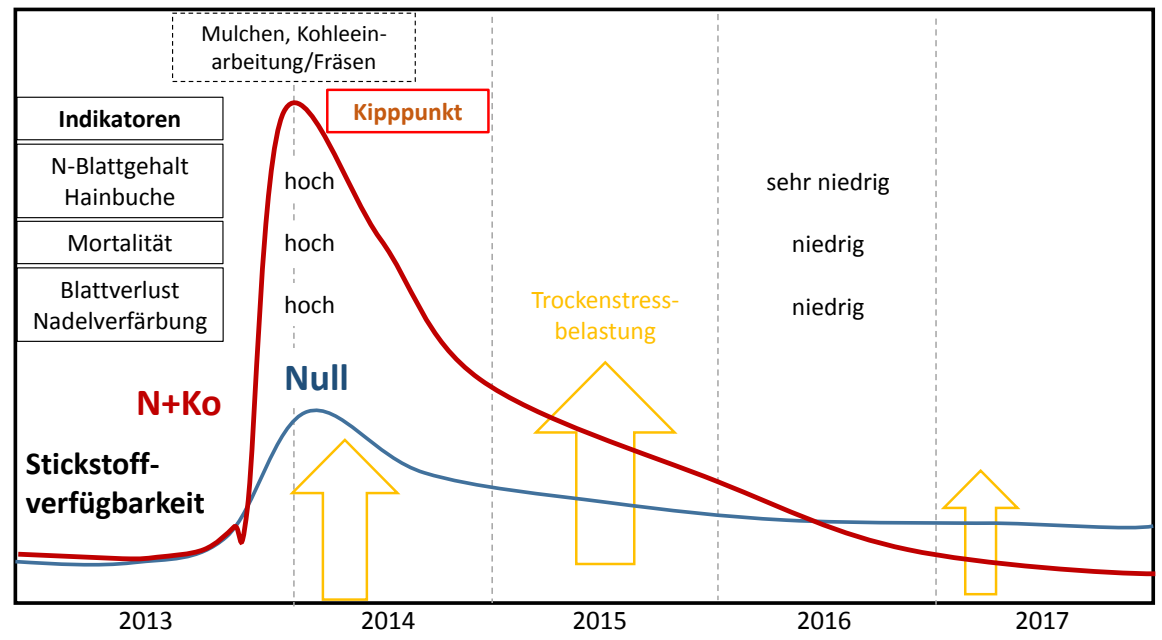

Abbildung 4.5: Dynamik von Störungen und Wirkungen

Das durch den Forstbetrieb favorisierte Ökosystem ist eine stabile künstliche Verjüngung, in der eine hinreichende Zahl an Forstpflanzen das Jungwuchsstadium bis zum Bestandesschluss überstehen (siehe Abb. 4.6). Mit zunehmender Zahl von Mortalitätsereignissen steigt die Gefahr einer weitgehend vergrasten Fläche (alternatives System). Ist dieser Zustand einmal erreicht, kann er als außerordentlich stabil angesehen werden, da durch den dichten Gras-Wurzelfilz kaum Wasser in tiefere Bodenschichten dringt. Der oberirdische Teil der Gras- und Krautvegetation konkurriert mit den gepflanzten Bäumen um Licht und Wuchsraum.

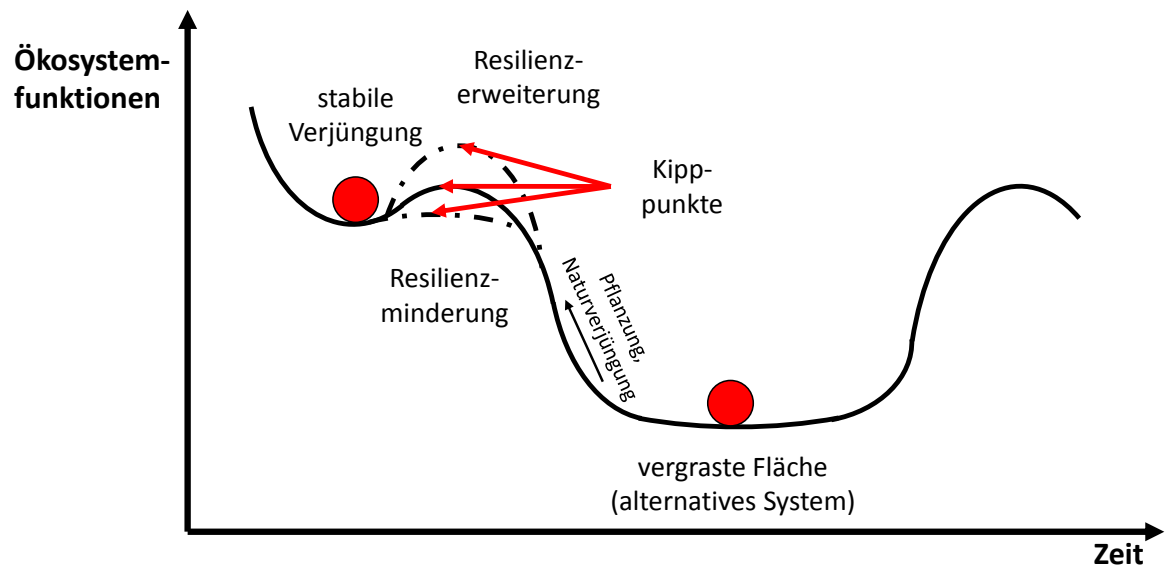

Abbildung 4.6: Systemzustand und Resilienzveränderungen am Beispiel des Feldversuches 
Gerät das Ökosystem einmal in diesen stabilen Zustand, kann es nur durch hohen Aufwand (Neupflanzung und regelmäßiges Freimähen) wieder in den gewünschten Zustand (Verjüngung aus Zielbaumarten) überführt werden. Eine Selbstregulierung, die Naturverjüngung, ist aus den beschriebenen Gründen wenig wahrscheinlich. Lediglich ausgesprochene Lichtbaumarten wie beispielsweise Birke oder Pappel können eine solche Fläche auf lange Sicht sukzessionsartig wieder bestocken. Dieses Alternativsystem ist fest etabliert, es besitzt eine starke Attraktorwirkung. Auch durch ein deutliches Absinken der Stickstoffverfügbarkeit geht das System nicht ohne weiteres in den ursprünglichen Zustand zurück.

Die 2016 gepflanzten Kiefern zeigen im Herbst 2017 trotz geringer Stickstoffbelastung und ausbleibender Trockenperiode in den Vegetationszeiten 2016 und 2017 Überlebensraten von weniger als 50 \%. Dieser Umstand kann als Ausdruck von veränderten Konkurrenzverhältnissen zwischen Begleitvegetation und Forstpflanzen angesehen werden. Die Anwuchsverhältnisse für junge Bäume haben sich mit dem Systemübergang verschlechtert. Ortsübliche Anwuchsprozente von über 70 \% (mdl. Mitteilung, Revierleiter) können nur auf Flächen mit weniger stark etablierter Grasvegetation erreicht werden.

Mit dem Übergang in einen anderen Systemzustand geht eine Änderung der durch das Ökosystem geleisteten Funktionen einher. Eine vergraste Fläche erfüllt die Funktion der zukünftigen Holzbereitstellung, die Schutzfunktionen wie etwa Klima- und Naturschutz und die Erholungsfunktion nicht mehr, oder nur noch unzureichend.

\subsubsection{Resilienzeigenschaften der Baumarten Hainbuche und Kiefer}

Die Hainbuche zeigt in diesem Versuch in den ersten beiden Versuchsjahren in allen Versuchsvarianten höhere Überlebensraten als die Kiefer. Die Hainbuche reagiert deutlich weniger sensitiv auf einen Stickstoffeintrag. Als Reaktion auf Trockenperioden ist bei einigen Pflanzen der Abwurf der gesamten Belaubung und das Zurücktrocknen großer Teile der oberirdischen Biomasse zu beobachten. Aber auch Absterbeereignisse sind festzustellen. Nach einer Trockenperiode reagieren einige Hainbuchen während der gleichen Vegetationszeit mit dem erneuten Austreiben von Blättern. Im nachfolgenden Jahr werden als modifikative Anpassung kleinere Blätter ausgebildet. Es kann außerdem ein hohes Stockausschlagsvermögen geschädigter Pflanzen beobachtet werden. Diese große Bandbreite der Reaktionen ist als Anpassungsstrategie an trockenere Bedingungen zu werten. Außerdem kann das hohe Vermögen des Stockausschlages und der Wiederaustriebs der Blätter der Hainbuche als hohes Restitutionsvermögen und damit als Zeichen hoher Resilienz bezeichnet werden.

Die Kiefer zeigt in dieser Arbeit eine hohe Sensitivität einem Stickstoffeintrag gegenüber, die die grundsätzlich hohe Angepassheit an trockene Wuchsbedingungen in diesem Versuch überprägt. Sie gilt als eine, an geringe Nährstoffversorgung und Trockenheit angepasste Pionierbaumart (Kätzel et al. 2015) und tritt vorwiegend in Vorwald- und frühen Zwischenwaldstadien auf (Eichhorn et al. 2016). Die Kiefer verfügt über eine effiziente Kombination von morphologischen Besonderheiten und einer breiten physiologischen/genetischen Reaktionsnorm. Nadelstruktur, die Regulation der Spaltöffnungen und der Aufbau des Wassertransportsystems ermöglichen ein vergleichsweise längeres Überleben unter Wassermangelbedingungen (Kätzel et al. 2008). Bei Kiefer beträgt der Wasserverlust bei geschlossenen Stomata (kutikuläre Transpiration) nur $14-25 \%$ des Wasserverlustes der Buche (Larcher 1994).

Das Anpassungspotenzial der Kiefer an zukünftige Klima- und Standortsbedingungen wird von Roloff und Grundmann (2008) als besonders hoch eingeschätzt, wenngleich Kätzel und Löffler (2007) eine nach Risiken differenzierte Betrachtung vorschlagen. Die in dieser Arbeit beobach- 
tete Anfälligkeit der Kiefer gegenüber einer Eutrophierung des Standortes sollte angesichts der anhaltend hohen atmosphärischen Stickstoffeinträge Anlass sein, das Anpassungspotenzial der Kiefer nicht zu überschätzen. Im Zuge stärkerer Stickstoffeinträge wird ein rückläufiger Anteil der Kiefer an der Waldfläche prognostiziert (Hofmann 2007).

\subsubsection{Die langfristige Entwicklung der Resilienz}

Der in dieser Arbeit ausgewertete Feldversuch betrachtet zunächst kurz- bis mittelfristig wirksame Effekte. Aus der Literatur sind jedoch auch langfristige Effekte der Kohleeinarbeitung bekannt (siehe Abschnitt 1.3 auf S. 17). Es deuten sich jedoch auch im Feldversuch langfristige Entwicklungen an. Die in Laboruntersuchungen nachgewiesene Erhöhung der nutzbaren Feldkapazität hat in der Initialphase des Feldversuches (2014 und 2015) nicht zu besseren Anwuchserfolgen geführt. Ab 2016 zeichnen sich jedoch bessere Anwuchsbedingungen (geringere Mortalität, besserer Blattzustand, höherer Zuwachs) zumindest für eine Kohlevariante ab (HKpur).

Das Potenzial der Kohlen, Stickstoff dauerhaft zu fixieren, scheint außerordentlich hoch zu sein. Wie sich dadurch die Stickstoffverfügbarkeit für die Pflanzen dauerhaft entwickelt, kann jedoch nicht abgeschätzt werden. 


\subsection{Methodendiskussion und Indikatorenauswahl}

Abbildung 4.7 zeigt die zeitliche Dynamik der Störungen Bodenbearbeitung, Stickstoffverfügbarkeit und Trockenstress sowie die Messtermine der erhobenen Indikatoren. Die hohe Dynamik der Stressoren macht deutlich, dass die Ergebnisse der Messungen immer im Zusammenhang mit der aktuellen Belastungssituation durch die Stressoren interpretiert werden müssen.

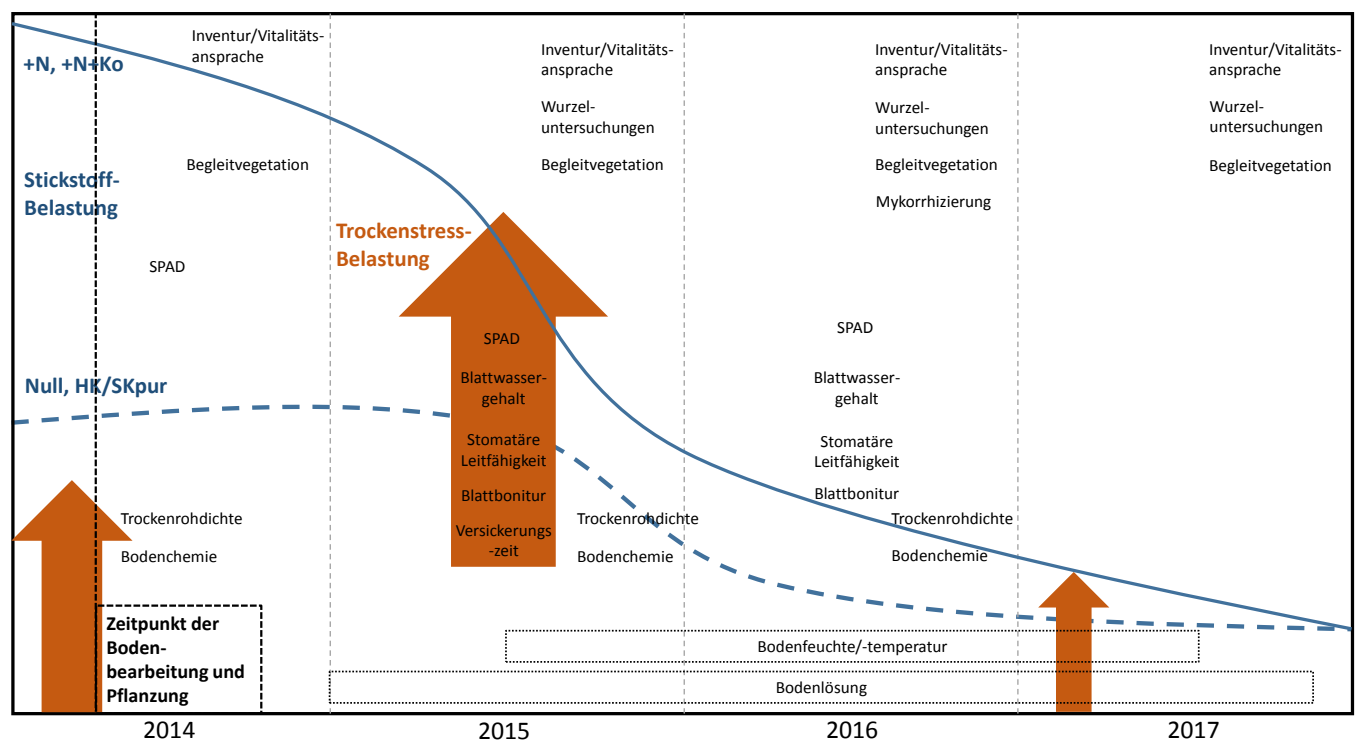

Abbildung 4.7: Zeitliche Darstellung der Störungen und die Messzeitpunkte der Indikatoren.

\subsubsection{Allgemeine Methodendiskussion}

Die Interpretation von komplexen Wechselwirkungen in Freilandversuchen gestaltet sich stets schwieriger als bei Laborexperimenten, die unter kontrollierten Bedingungen nach dem Ceterisparibus-Prinzip durchgeführt werden. Die auftretenden Effekte im Feldversuch können durch eine Überlagerung verschiedener Prozesse bzw. Wirkung mehrerer Faktoren nur schwer mit einzelnen Einflussgrößen in Bezug gesetzt werden (Murach und Wiedemann 1988). Der Feldversuch in dieser Arbeit ist gut geeignet langfristige Anpassungsprozesse der Baumarten zu untersuchen. Der Topfversuch bietet Gelegenheit, Einblicke in die kurzfristigen Reaktionen der Pflanzen gegenüber den versuchsbedingten Störungen zu gewinnen.

Der Pflanzenauswahl kommt bei stichprobenbasierten Messungen eine besonders hohe Bedeutung zu. Im Rahmen dieser Arbeit erfolgte die Auswahl der Pflanzen für stichprobenbasierte Messungen streng zufallsbasiert. Bei Verfahren ohne Zufallsauswahl würden sonst systematische und methodische Fehler entstehen (Sachs und Hedderich 2006).

Bei der Gewinnung von Probenmaterial (Boden, Pflanzengewebe) werden häufig aus Einzelproben Mischproben gebildet. Dies geschieht aus einer Vielzahl von Gründen. Bei der Blattprobenahme, um genug Material für die weitere Analyse zu erhalten. Bei der Bodenprobenahme, um eine zeit- und kosteneffiziente Beprobung einer große Fläche zu realisieren. Bei den Messergebnissen handelt es sich allerdings um Durchschnittswerte, die natürliche Variabilität der Grundgesamtheit in zeitlicher sowie räumlicher Hinsicht geht in dieser Stichprobe verloren.

Bei der Untersuchung an Pflanzen unter Stress muss berücksichtigt werden, dass nur Pflanzen 
in die Auswahl für die Messung gelangen können, die bis zum Zeitpunkt der Messung überlebt haben. Individuen, die bereits abgestorben sind, können nicht mehr Teil der Stichprobe oder der Vollaufnahme sein. Messungen des aktuellen Stresszustandes werden von diesem Effekt beeinflusst. Zusätzlich entsehen durch höhere Mortalitätszahlen einzelner Versuchsvarianten unterschiedlich große Grundgesamtheiten. Bei zu großen Unterschieden in der Grundgesamtheit bzw. zu kleinen möglichen Stichproben wird auf eine weitergehende statistische Auswertung verzichtet. Dies betrifft die Auswertung der 2014 und 2015 gepflanzten Kiefer. Um den Versuch weiterhin wissenschaftlich betreuen zu können, wurde nach den massiven Mortalitäten bei der Kiefer entschieden, im jeweils folgenden Frühjahr (2015 bzw. 2016) neu zu pflanzen.

\subsubsection{Indikatorenauswahl}

Indikatoren, die Umweltbedingungen und Pflanzenreaktionen in dieser Arbeit gut abgebildet haben, sind in Tabelle 3.15 aufgeführt.

Die Gewinnung von Bodenlösung wurde im Winter und Frühjahr 2015 nach dem Verfahren von Wilpert et al. (2011) durchgeführt. Dabei wird für 3-4 Tage Unterdruck an eine Saugkerze angelegt und Bodenlösung gewonnen. Im sandigen Substrat des Standortes ist dabei eine starke Abhängigkeit von Regenereignissen gegeben, um genügend Bodenlösung zu gewinnen (Unold 2000). Bei zukünftigen Versuchen sollten die Saugkerzen bereits bei Versuchsanlage an ihre endgültigen Standorte installiert werden, um eine ausreichende Konditionierung zu gewährleisten und so früh wie möglich Bodenlösung gewinnen zu können. Mit diesem Vorgehen würden Informationen zur Konzentration der Bodenlösung auch für die Initialphase des Feldversuches vorliegen.

Die Modellierung der durchschnittlichen Stickstoffvorräte in der Bodenvegetation auf den Versuchsparzellen basierte auf den Ergebnissen der Begleitvegetationsinventur. Nach Mölder et al. (2008) könnte die Biomasse der Begleitvegetation durch das Modell tendenziell unterschätzt werden. Die Stickstoffgehalte je Artengruppe sind weitgehend in geschlossenen Waldbeständen ermittelt worden. Trotz erfolgter Anpassungen an Kahlschlagsituationen durch Klinck und Fröhlich (2009) und Heinrichs et al. (2010) stellen die Vorratsmengen an Stickstoff wahrscheinlich eher eine Unterschätzung als eine Überschätzung dar.

Die Blatt-/Nadelelementgehalte und -verhältnisse sind sensible Indikatoren für die Versorgungslage der Pflanzen mit Nährelementen. Ausreichend hohe Nährstoffgehalte und harmonische Nährstoffverhältnisse sind für ein gesundes Wachstum unverzichtbar (Flückiger und Braun 2003). Die Nährstoffgehalte spiegeln grundsätzlich die Nährstoffverfügbarkeit im Boden wider, wobei Bäume die Nährstoffaufnahme selektiv steuern können, um die Aufnahme bestimmter Elemente zu bevorzugen oder zu hemmen (Marschner 1995). Die Stickstoffgehalte in Blättern und Nadeln unterliegen jährlichen und jahreszeitlichen Schwankungen, die mit variierenden Streuzersetzungsund Stickstoffmineralisierungsraten zusammenhängen (Evers 1972; Asche 1997). Daher werden für den Jahresvergleich Probenahmen an jeweils gleichen Terminen miteinander verglichen.

Die indirekte Messung des Blattstickstoffgehaltes per SPAD-Messung kann nur grobe Hinweise auf den aktuellen Stickstoffernährungsstatus von Hainbuchen geben. Das Messverfahren wird vorwiegend für die Ermittlung des Stickstoff-Düngebedarfes von landwirtschaftlichen Kulturen angewendet und weist für die forstliche Anwendung eine ungenügende Differenzierung im unteren Messbereich auf. Lediglich bei sehr großen Unterschieden in der Blatt-Stickstoffkonzentration (siehe Topfversuch) können Rückschlüsse auf die Stickstoffernährung gezogen werden. Vorteile des Messverfahrens ist die kurze Messzeit, die eine hohe Wiederholungszahl ermöglicht. Unterstützende chemische Analysen sind jedoch nach wie vor notwendig, um den Ernährungszustand nach empirischen Schwellenwerten einzuordnen.

Die Untersuchung der Feinwurzelmykorrhizierung fand im Herbst 2016 statt. Die Hainbuchen waren zu diesem Zeitpunkt bereits seit drei Vegetationsperioden, die Kiefern seit einer Vege- 
tationsperiode im Versuchsfeld. Um die Auswirkungen der Störungen auf die Mykorrhizierung zu beobachten, sind Untersuchungen während der Anfangsphase des Versuches sinnvoll, da die Mykorrhizagemeinschaft schnell auf veränderte Umweltbedingungen reagiert (Rapp 1991).

Die Ergebnisse der Messungen von Spross- und Wurzeltrockenmassen ist in dieser Arbeit ein sensitiver Indikator für die Biomasseallokation von Pflanzen und gibt Einblicke in die Anpassungsstrategien gegenüber Störungen.

Großer Vorteil der Messung der stomatären Leitfähigkeit ist die nicht-destruktive Messung von intakten Blattorganen in natürlicher Exposition.

Insgesamt stehen in dieser Arbeit zur Beurteilung der Vitalität der Kiefer weniger Indikatoren als für die Hainbuche zur Verfügung. Dies liegt einerseits an fehlenden Messverfahren. So sind stomatäre Leitfähigkeit und SPAD-Gehalt an Kiefernnadeln ohne entsprechend angepasste Aufsätze nicht zu erfassen. Diese Aufsätze standen nicht zur Verfügung. Zum anderen wirken sich Nadelproben an Kiefern deutlich destruktiver aus als an Hainbuchen. Um eine für die Analyse hinreichende Menge zu gewinnen, ist ein großer Teil der Nadelmasse des Jahrestriebes einer Pflanze zu entfernen. Dies bedeutet für die Pflanze große Einbußen in Zuwachs und Vitalität. Aus diesen Gründen ist die Datenlage zur Beurteilung des physiologischen Stresszustandes im Vergleich zur Hainbuche eingeschränkt.

Darüberhinaus sind weitere Indikatoren und Messverfahren in dieser Arbeit angewendet worden, die die Umweltbedingungen und Pflanzenreaktionen weniger gut dargestellt haben oder eine hohe Fehleranfälligkeit besitzen. Die Ergebnisse sind zum Teil nicht in dieser Arbeit aufgeführt.

Die TDR-Bodenfeuchtesensoren liefern trotz vorheriger Prüfung zum Teil unplausible Werte. Nach Regenereignissen fehlte ein entsprechender Anstieg der Bodenfeuchte im Oberboden. Durch den hohen Anteil an organischer Substanz im Oberboden kann es zu Messfehlern durch das TDR-Verfahren kommen (Scherzer et al. 1996). Bei Trockenrohdichten von unter 1,2 $\mathrm{g} \mathrm{cm}^{-3}$, wie im Oberboden der Kohlevarianten, wird der Feuchtegehalt des Bodens unterschätzt. Luftspalten zwischen Boden und Sensor produzieren ebenfalls Messabweichungen. Das Vorhandensein von präferentiellen Fließwegen für Wasser kann zu einer unregelmäßigen horizontalen Verteilung der Bodenfeuchte führen. Diese Faktoren sorgen dafür, dass die Messwerte einiger TDR-Sensoren im Oberboden $(0-30 \mathrm{~cm})$ kaum mit den Werten der gravimetrischen Wassergehaltsbestimmung in diesem Horizont korrelieren. Diese Erkenntnisse haben dazu geführt, die gravimetrische Bestimmung des Wassergehaltes zu intensivieren.

Die Ermittlung der Wurzellängen ist sehr zeitaufwändig und korelliert stark mit den Trockenmassen der jeweiligen Wurzelkompartimente.

Die Wurzelausbreitung in horizontaler und vertikaler (Durchwurzelungstiefe) Richtung weist in dieser Untersuchung sehr hohe Streuungen zwischen den Individuen auf. Die Ergebnisse sind daher nicht aussagekräftig.

Für die biochemische Untersuchung der Blattgewebe müssen die Pflanzenteile sofort nach der Probenahme eingefroren werden und bis zur Gefriertrocknung tiefgefroren bleiben. Die Ergebnisse vom Juli 2015 und Juli 2016 geben Hinweise auf Zersetzungsprozesse, die auf unzureichend gekühlte Proben zurückzuführen sind.

Ein großer Nachteil der Blattwasserpotenzialmessung mittels Scholanderverfahren ist die hohe Destruktivität der Messung. Außerdem beschränkt die Messdauer die Anzahl der möglichen Proben im Beprobungszeitraum vor Sonnenaufgang (predawn).

Das Blattwasserdefizit korelliert stark mit dem Blattwassergehalt. Die Messung ist jedoch wesentlich aufwändiger und fehleranfälliger (Methodenbeschreibung in Steubing und Fangmeier (1992)). 


\section{Zusammenfassung}

Der, dieser Arbeit zugrunde liegende, Feldversuch wurde Anfang des Jahres 2014 im Hessischen Ried im Forstamt Lampertheim (Südhessen) angelegt. Mit dem Ziel der Stabilisierung einer forstlichen Verjüngung während Trockenphasen wurde Biokohle in den Oberboden eingearbeitet. Der Anwuchserfolg der gepflanzten Hainbuchen und Kiefern sollte im Vergleich zum ortsüblichen Verfahren gesteigert werden.

Der durch das sandige Ausgangssubstrat geprägte Standort zeichnet sich durch eine niedrige Wasserhaltekapazität und einen geringen Stickstoffvorrat aus. Der pH-Wert liegt meist über 6,2 und ist im kalkhaltigen Ausgangssubstrat aus Flugsand begründet. In einem randomisierten Blockversuch wurden sechs verschiedene Kohlevarianten mit je $80 \mathrm{t} \mathrm{ha}^{-1}$ ausgebracht und auf $25 \mathrm{~cm}$ Bodentiefe eingefräst. Auf jeweils 6 Wiederholungsparzellen wurde Hainbuche und Kiefer streifenweise gepflanzt. Während der Versuchsdauer konnten Trockenphasen zum Pflanzzeitpunkt 2014, in der Vegetationszeit 2015 und Anfang 2017 verzeichnet werden. Zwischen 2014 und 2017 erfolgten jährliche Inventuren, Boden- und Pflanzenuntersuchungen. Die Bodenlösung wurde ab 2015 beprobt. Um die zum Teil unerwarteten Ergebnisse zu verifizieren, wurden ein Extraktions- und ein Topfversuch unter kontrollierten Umweltbedingungen durchgeführt.

Der in der Begleitvegetation gebundene Vorrat an Stickstoff wurde mit PhytoCalc, die Sickerwassermengen mit LWF-Brook90 modelliert.

\subsection{Die wichtigsten Ergebnisse}

Die Bodenbearbeitung zur Versuchsanlage (Mulchen und Fräsen) hat den Oberboden aufgelockert. Der Prozess der Bodensetzung dauert mehr als 2 Jahre an. Die Trockenrohdichten im Oberboden der Kohlevarianten weisen nach zwei Jahren um 0,1-0,4 $\mathrm{g} \mathrm{cm}^{-3}$ niedrigere Werte als die Nullvariante auf. Die Senkung der Trockenrohdichten im Oberboden ist als langfristiger Effekt zu betrachten und kann sogar noch weiter fortschreiten (pure Kohlevarianten).

Die nutzbare Feldkapazität bis $90 \mathrm{~cm}$ Bodentiefe erhöht sich durch den Eintrag von Biokohle um bis zu 25 \%. Dies wird im Wesentlichen durch eine Erhöhung des Porenvolumens bewirkt. Im Oberboden liegen die Bodenwassergehalte der kohlebehandelten Parzellen im Mittel über denen der Nullvariante. Im Unterboden ist durch reduzierte Sickerwassermengen der gegenteilige Trend zu verzeichnen. Die Sickerwassermengen unter den kohlebehandelten Versuchsparzellen betragen im Schnitt nur zwischen 50 und 70 \% der Mengen unter den Nullparzellen.

Im Feld- und Topfversuch zeigen sich Auswirkungen von hydrophoben Eigenschaften der Kohlesubstrate, die die positiven Retentionseigenschaften im Feldeinsatz schmälern können. Ein durch den Kohleneintrag verursachter erhöhter Deckungsgrad der Begleitvegetation hat Auswirkungen auf weitere Komponenten des Wasserhaushaltes wie Evapotranspiration, Interzeption und Infiltration. Entscheidend für die günstigen Wirkungen auf die Pflanzen ist die Aufsättigung der Kohlen zu Beginn der Vegetationszeit bzw. zum Pflanzzeitpunkt.

Das Fräsen des Oberbodens zur Kohleeinarbeitung kurz vor der Pflanzung in Verbindung mit dem späten Pflanzzeitpunkt zeigt negative Auswirkungen auf das Anwuchsverhalten der Kiefer. Das Mulchen, als typisches Kulturvorbereitungsverfahren im Forstamt Lampertheim, führt zu mittleren Anwuchsprozenten von etwa 70 \% im Jahr 2014 für die Kiefer (mdl. Mitteilung durch Revierleiter). Im vorliegenden Versuch beträgt die Überlebensrate im Jahr 2014 nur $13 \%$ (Nullvariante). 
Durch die Nachbehandlung mit Ammoniumsulfat von einigen Kohlevarianten ergibt sich ein Stickstoffeintrag von bis zu $1133 \mathrm{~kg} \mathrm{ha}^{-1}$. Der massive Stickstoffeintrag in dieser Größenordnung war nicht beabsichtigt. Die applizierte Kohlemenge ergab sich aus der beabsichtigten Erhöhung der nutzbaren Feldkapazität. Die durch die Nachbehandlung erreichten Stickstoffgehalte sind für landwirtschaftliche Anwendungen optimiert, in Kombination mit der hohen Eintragsmenge der Kohle jedoch viel zu hoch. Die Stickstoffverfügbarkeit erhöht sich deutlich, dies zeigen die Konzentrationen in der Bodenlösung und in den Pflanzengeweben während der Anfangsphase des Feldversuches in den Jahren 2014 und 2015. Eine stimulierte Mineralisierung der organischen Substanz, hervorgerufen durch „Priming-Effekte“ der Biokohlen, kann in diesem Zeitraum im Feldversuch festgestellt werden. In der zweiten Phase des Feldversuches ab 2016 können kaum Unterschiede in der Stickstoffverfügbarkeit festgestellt werden. Das sehr hohe Fixierungspotenzial der Biokohlen für Stickstoff wird mit einem hohen chemischen und physikalischen Sorptionspotenzial erklärt.

Die beobachteten Auswirkungen der erhöhten Stickstoffverfügbarkeit in der Initialphase (2014 und 2015) auf die Pflanzen sind zahlreich. So können in der Begleitvegetation deutlich höhere Deckungsgrade und mehr nitrophile Arten festgestellt werden. Die hohe Mortalität der Bäume der stickstoff- $(+\mathrm{N})$ und kompost- $(+\mathrm{N}+\mathrm{Ko})$ nachbehandelten Kohlevarianten liefert Hinweise auf eine pflanzentoxische Konzentration von Stickstoffverbindungen während der Anfangsphase des Versuches. Die Varianten der puren Holzkohle und der Nullvariante weisen im Feldversuch die höchsten Überlebensraten auf. Diese Varianten zeigen zudem einen besseren Zustand der Benadelung/Belaubung. Morphologische Auswirkungen einer erhöhten Stickstoffverfügbarkeit wie höhere Spross-Wurzel-Verhältnisse, größere Blattflächen und vermindertes Feinwurzelwachstum können beobachtet werden.

Im Sommer 2015 wird eine hohe physiologische Trockenstressbelastung der Hainbuchen festgestellt. Unterschiede zwischen den Varianten zeigen sich dabei nicht. Lediglich während moderater Trockenstresssituationen im Sommer 2016 weisen die Pflanzen der Nullvariante höhere Trockenstressbelastungen auf. Die durch Trockenstress ausgelöste Reaktionskaskade wird näher dargestellt und Unterschiede in den Überlebensstrategien werden diskutiert. Dabei können entsprechende Stresszustände sowohl als Belastungs- wie auch als Anpassungsreaktionen interpretiert werden. Die Unterscheidung in kurz- oder langfristige Folgeerscheinungen ist für eine Bewertung der pflanzenschädlichen Wirkung ausschlaggebend.

Die durch die hohe Stickstoffverfügbarkeit hervorgerufenen Pflanzenreaktionen resultieren in einer herabgesetzten Resistenz gegenüber klimatischen Belastungen wie Trockenstress.

Im Baumartenvergleich weist die Kiefer eine deutlich höhere Sensitivität gegenüber der Stickstoffverfügbarkeit auf. Dies wird mit der Pioniereigenschaft und der damit verbundenen Anpassung an nährstoffarme Standorte erklärt.

Für die stickstoff- $(+\mathrm{N})$ und kompost- $(+\mathrm{N}+\mathrm{Ko})$ nachbehandelten Kohlevarianten wird nach drei Jahren eine höhere Stickstoffspeicherung durch die Kohlen bilanziert. Die Speicherwirkung kann zum einen mit der Sorptionskapazität der Kohlen und zum anderen mit einer möglicherweise erhöhten mikrobiellen Immobilisierung begründet werden. Ob die Stickstoffspeicherung als Effekt der Nachbehandlung eine langfristige Wirkung entfaltet, kann nach der 4-jährigen Versuchsdauer noch nicht abgeschätzt werden.

Die Pflanzen der puren Holzkohlevariante zeigen in vielen untersuchten Parametern ähnliche Eigenschaften wie die Pflanzen der Nullvariante. Möglicherweise verändert diese Kohlevariante den Stickstoffhaushalt im Vergleich zur Nullvariante weniger als andere Kohlevarianten.

Trotz teilweise höherer Trockenstressbelastung der Hainbuchen der Nullvariante (Kaliumgehalt, stomatäre Leitfähigkeit, Blattwassergehalt), weist die Nullvariante zusammen mit der puren Holzkohlevariante (HKpur) die höchsten Überlebensraten auf. Die spricht für eine erfolgreiche Anpassung während der Trockenstressphasen. 


\subsection{Hypothesendiskussion}

Durch die Biokohleeinarbeitung wird die Wasserhaltekapazität des Oberbodens wesentlich erhöht. Die erforderliche Kohlemenge ist so bemessen, dass eine Erhöhung der pflanzennutzbaren Feldkapazität um $15 \%$ erreicht wird (1).

Diese Hypothese kann anhand zahlreicher Befunde bestätigt werden (siehe Abschnitt 4.1). Die positiven Wirkungen der Biokohle zeigen sich in einer bis zu 19 Vol.-\% höheren nutzbaren Feldkapazität (nFK) im Oberboden. Auf den Bodenkörper bis $90 \mathrm{~cm}$ Bodentiefe erhöht sich die nutzbare Feldkapazität zwischen $16 \%$ und $25 \%$. Dies übersteigt die anvisierte Erhöhung. Für eine Erhöhung der nFK um 15 \% wäre für die Variante HK+N+Ko nur ein Kohleeintrag von etwa $66 \mathrm{t} \mathrm{ha}^{-1}$ statt $80 \mathrm{t} \mathrm{ha}^{-1}$ notwendig gewesen.

Ausschlaggebend für die verbesserte Retentionsfähigkeit ist das hohe Porenvolumen der Biokohle. Eine damit einhergehende Absenkung der Trockenrohdichte und die mögliche Bildung hydrophober Bodenoberflächen mit dem Auftreten von präferentiellen Fließwegen für Wasser können die positiven Wirkungen im Feldeinsatz jedoch abschwächen. Wichtige Voraussetzungen für eine positive Wirkung sind aber die Aufsättigung der Kohle mit Wasser zum Zeitpunkt der Pflanzung und eine ausreichende Bodensetzung nach Bodenbearbeitung. Dies war angesichts der zeitlichen Abfolge in der Anlage des Feldversuchs nicht gegeben. In späteren Versuchsjahren wirkt sich die Biokohle stressmildernd während Trockenperioden aus. Dies zeigen physiologische Trockenstressindikatoren für die Hainbuchen der kohlebehandelten Parzellen.

Durch die chemische und biologische Nachbehandlung der Kohlen werden die chemischen Eigenschaften des Oberbodens nach Kohleneinarbeitung deutlich verändert (2).

Mit der Nachbehandlung der stickstoff- $(+\mathrm{N})$ und kompost- $(+\mathrm{N}+\mathrm{Ko})$ nachbehandelten Kohlevarianten geht ein enormer Stickstoffeintrag von bis zu $1133 \mathrm{~kg} \mathrm{ha}^{-1}$ einher. Der Kohlenstoffeintrag beträgt zwischen 28 und $69 \mathrm{t}$ ha ${ }^{-1}$. Damit verändern sich die Stickstoff- und Kohlenstoffvorräte im Oberboden $(0-30 \mathrm{~cm})$ wesentlich. In den ersten beiden Versuchsjahren lassen sich erhöhte Stickstoffkonzentrationen im Sickerwasser der stickstoff- $(+\mathrm{N})$ und kompost- $(+\mathrm{N}+\mathrm{Ko})$ nachbehandelten Kohlevarianten beobachten. Die Wuchsdynamik und das Artenspektrum der Begleitvegetation weisen ebenfalls auf eine massiv erhöhte Stickstoffverfügbarkeit auf den Parzellen der stickstoff- $(+\mathrm{N})$ und kompost- $(+\mathrm{N}+\mathrm{Ko})$ nachbehandelten Kohlevarianten in den ersten beiden Versuchsjahren hin.

Die gesamten Austragsmengen an Stickstoff sind angesichts der sehr hohen Eintragsmenge aber als sehr gering zu bezeichnen. In den Folgejahren liegen die Stickstoffausträge dieser Varianten (mit Ausnahme von Variante HK+N) unter denen der Nullvariante und der puren Kohlevarianten, was auf ein ansteigendendes Potenzial zur Stickstoffimmobilisierung schließen lässt.

Die Pflanzen auf den Parzellen der Biokohlevarianten weisen höhere Überlebenszahlen als die Pflanzen der Nullvariante auf (3).

Diese Hypothese muss für den betrachteten Versuchszeitraum in großen Teilen abgelehnt werden. Im Vergleich zur Nullvariante zeigen die Hainbuchen und die 2014 bzw. 2015 gepflanzten Kiefern der stickstoff- $(+\mathrm{N})$ und kompost- $(+\mathrm{N}+\mathrm{Ko})$ nachbehandelten Kohlevarianten ein deutlich schlechteres Anwuchsverhalten mit geringeren Überlebenszahlen. Lediglich die pure Holzkohlevariante (HKpur) zeigt ähnliche Überlebenszahlen wie die Hainbuchen der Nullvariante. Bei den 2014 und 2015 gepflanzten Kiefern dieser Variante zeigen sich etwas bessere Anwuchszahlen als bei den Kiefern der Nullvariante.

Gründe für das schlechtere Anwuchsverhalten auf den Parzellen der stickstoff- $(+\mathrm{N})$ und kompost$(+\mathrm{N}+\mathrm{Ko})$ behandelten Kohlevarianten liegen in der deutlich erhöhten Stickstoffverfügbarkeit in 
der Initialphase des Versuches (2014 und 2015). Entsprechende Indikatoren wie die Stickstoffkonzentration in der Bodenlösung, die Blatt- und Nadelgehalte und das Aufkommen und die Artenzusammensetzung der Begleitvegetation zeigen einen Überfluss an Stickstoff an.

Die Reaktionen der Pflanzen auf die Stickstoffüberversorgung sind vielfältig und reichen von Blatt- und Wurzelveränderungen bis hin zur Mortalität. So zeigen Kiefern der stickstoffnachbehandelten $(+\mathrm{N})$ Kohlevarianten im Topfversuch noch während der Bewässerungsphase erste Absterbeerscheinungen. Auch die Kiefern der kompost- $(+\mathrm{N}+\mathrm{Ko})$ nachbehandelten Kohlevarianten zeigen im Vergleich zu den puren Kohlevarianten (HKpur und SKpur) reduzierte Überlebensdauern während der Trockenphase. Im Feldversuch sind auf den Parzellen der kompost- $(+\mathrm{N}+\mathrm{Ko})$ behandelten Kohlevarianten in den ersten beiden Versuchsjahren Komplettausfälle der Kiefern zu beobachten.

Im Zusammenhang mit dem im Feldversuch aufgetretenem Trockenstress, wurde die Trockenresistenz der Pflanzen auf den Parzellen der stickstoff- $(+\mathrm{N})$ und kompost- $(+\mathrm{N}+\mathrm{Ko})$ behandelten Kohlevarianten soweit herabgesetzt, dass in den ersten beiden Versuchsjahren ein Großteil der Pflanzen dieser Varianten abgestorben ist.

\subsection{Unterschiede zwischen Holz- (HK) und Spelzenkohle (SK)}

Die Spelzenkohle besitzt eine deutlich geringere Trockenrohdichte als das Holzkohlesubstrat, dem entsprechend liegen die Trockenrohdichten im Oberboden der SK-Varianten unter denen der Holzkohlevarianten. Die Retentionskurven beider Substrate verlaufen fast deckungsgleich, es kann daher ein identischer Beitrag zur nutzbaren Feldkapazität angenommen werden. Die hydrophobe Eigenschaft der trockenen Kohle scheint bei der puren Spelzenkohle im Vergleich zur Holzkohle etwas stärker ausgeprägt zu sein.

Im Vergleich mit der puren Holzkohle wurde mit der puren Spelzenkohle mehr als die dreifache Stickstoffmenge in den Oberboden eingebracht. Dies wird durch die Stickstoffausträge bestätigt, die im ersten Jahr des Versuches zum Teil deutlich über denen der puren Holzkohlevariante liegen. Während der ersten Vegetationszeit lässt sich die höhere Stickstoffverfügbarkeit durch die Spelzenkohle anhand höherer Deckungsgrade der Begleitvegation, größerer Blattflächen der Hainbuchen, höherer Spross-Wurzel-Verhältnisse und geringerer Wurzelbiomasse der Hainbuchen ablesen.

Die Spelzenkohlen sind deutlich poröser, sie weisen eine etwa dreifach höhere innere Oberfläche als die Holzkohle auf. Dies lässt langfristig ein höheres Immobilisierungspotential für Stickstoff durch Ad- und Absorption vermuten. Auch die Pflanzenreaktionen weisen ab 2015 auf ein wachsendes Fixierungspotenzial der Spelzenkohle hin. So zeigen die Stickstoffgehalte der Hainbuchenblätter ab dem Jahr 2015 im Vergleich zur Holzkohle geringere Werte.

\subsection{Rückschlüsse für die forstliche Praxis und Ausblick}

Die Überlebensraten der Kiefern der Nullvariante im Jahr 2014 geben Anlass das Fräsen als kulturvorbereitende Maßnahme unmittelbar vor der Pflanzung kritisch zu hinterfragen. Die Auflockerung des Oberbodens bis in eine Tiefe von $20-30 \mathrm{~cm}$ durch das Mulchen sollte mindestens ein halbes Jahr vor der Pflanzung ausgeführt werden, damit sich der Boden zum Zeitpunkt der Pflanzung setzen kann. Auch aufgrund der erforderlichen Aufsättigungszeit sollte die Kohleeinarbeitung zu Beginn der Nichtvegetationszeit erfolgen, damit sich die Biokohle bis zum Beginn der Vegetationszeit maximal aufsättigen kann und die volle nutzbare Feldkapazität zum Pflanzzeitpunkt zur Verfügung steht. 
Die Nachbehandlung der Kohlen mit Stickstoff und Kompostierung hat keine positiven Effekte auf das Anwuchsverhalten von Hainbuchen und Kiefern gezeigt. Im Gegenteil, die Anwuchsbedingungen haben sich in den ersten beiden Versuchsjahren zum Teil deutlich verschlechtert (Stickstoffverfügbarkeit, Konkurrenz durch Begleitvegetation). Daher lautet die Empfehlung nur pure Kohlensubstrate zu verwenden.

In der Initialphase des Versuches (2014 und 2015) zeigen auch die Parzellen der puren Kohlesubstrate eine enorme Dynamik in der Stickstoffverfügbarkeit durch veränderte Mineralisationsund Immobilisierungsprozesse. Die Kulturbegründung sollte daher idealerweise mindestens zwei Jahre nach einer Kohleeinarbeitung erfolgen auch wenn dies in der forstlichen Praxis schwer zu realisieren ist.

Je geringer der Tongehalt des Ausgangssubstrats, umso höher kann die positive Wirkung der Kohlen auf die nutzbare Feldkapazität sein. Daher ist im Vorfeld der Kohleeinarbeitung eine präzise Substratkartierung von in Frage kommenden Flächen unerlässlich. Dies dient der Abschätzung der notwendigen Kohlemengen für eine angestrebte Verbesserung der nutzbaren Feldkapazität. Aufgrund der hohen Kosten von Herstellung, Transport und Einarbeitung der Kohlesubstrate ist eine Kosten-Nutzen-Abwägung unerlässlich. Der Einsatz von Biokohlen sollte hinsichtlich des Geländewasserhaushaltes nur auf den schwächsten Standorten erfolgen. Dies sind Standorte aus ton- und schluffarmen Sandsubstraten und fehlendem Grundwasseranschluss.

Das hohe Immobilisierungspotenzial der Biokohle für Stickstoff erlaubt auch andere Anwendungsszenarien als die Kulturbegründung. So kann der Nitrataustrag mit dem Sickerwasser deutlich reduziert werden. Aus Gründen des Grundwasserschutzes kann daher eine räumlich begrenzte Anwendung sinnvoll erscheinen.

Die Versuchsfläche im Forstamt Lampertheim sollte auch nach Ablauf des Projektes als Versuchsfläche zur Verfügung stehen, da erhebliche Investitionen zur Versuchsanlage geleistet wurden. Auch zukünftig können Bonitätsparameter der Pflanzen aufgenommen werden, um Veränderungen in der Wuchsdynamik und positive wie negative Wirkungen der Kohlen zu erfassen und zu bewerten. Ein Aufnahmeturnus von etwa 3 bis 5 Jahren sollte genügen, da sich die Verhältnisse auf dem Versuchsfeld weitgehend stabilisiert haben. 


\section{Literatur}

Abel, Stefan, Andre Peters, Steffen Trinks, Horst Schonsky, Michael Facklam und Gerd Wessolek (2013). "Impact of biochar and hydrochar addition on water retention and water repellency of sandy soil". In: Geoderma 202, S. 183-191.

Aber, John D, Jerry M Melillo, Knute J Nadelhoffer, Charles A McClaugherty und John Pastor (1985). "Fine root turnover in forest ecosystems in relation to quantity and form of nitrogen availability: a comparison of two methods". In: Oecologia 66.3, S. 317-321.

Aber, John D, Knute J Nadelhoffer, Paul Steudler, Jerry M Melillo et al. (1989). "Nitrogen saturation in northern forest ecosystems." In: BioScience 39.6, S. 378-286.

AG-Boden (2005). Bodenkundliche Kartieranleitung:[KA 5]; mit 103 Tabellen und 31 Listen. Schweizerbart.

Ahmad, Mahtab, Anushka Upamali Rajapaksha, Jung Eun Lim, Ming Zhang, Nanthi Bolan, Dinesh Mohan, Meththika Vithanage, Sang Soo Lee und Yong Sik Ok (2014). "Biochar as a sorbent for contaminant management in soil and water: a review". In: Chemosphere 99, S. 1933 .

Amonette, James E und Stephen Joseph (2009). "Characteristics of biochar: microchemical properties". In: Biochar for environmental management: Science and technology 33.

Anders, S, W Beck, A Bolte, G Hofmann, M Jenssen, UK Krakau und J Müller (2002). Ökologie und Vegetation der Wälder Nordostdeutschlands-Einfluss von Niederschlagsarmut und erhöhtem Stickstoffeintrag auf Kiefern-, Eichen-, und Buchen- Wald- und Forstökosysteme des nordostdeutschen Tieflandes. Kessel.

Angeles, Guillermo, Barbara Bond, John S Boyer, Tim Brodribb, J Renée Brooks, Michael J Burns, Jeannine Cavender-Bares, Mike Clearwater, Hervé Cochard und Jonathan Comstock (2004). "The cohesion-tension theory". In: New Phytol 163.3, S. 451-452.

Angst, Teri E und Saran P Sohi (2013). "Establishing release dynamics for plant nutrients from biochar". In: GCB Bioenergy 5.2, S. 221-226.

Asche, N (1997). "Nährelementgehalt in Buchenblättern unter besonderer Berücksichtigung der zeitlichen Variation auf basenarmen Standorten in Nordrhein-Westfalen". In: Forstwissenschaftliches Centralblatt 116.1-6, S. 394-402.

Atkinson, Christopher J, Jean D Fitzgerald und Neil A Hipps (2010). "Potential mechanisms for achieving agricultural benefits from biochar application to temperate soils: a review". In: Plant and soil 337.1-2, S. 1-18.

Bachmann, Jörg, Jiem Krueger, Marc-O Goebel und Stefanie Heinze (2016). "Occurrence and spatial pattern of water repellency in a beech forest subsoil". In: Journal of Hydrology and Hydromechanics 64.2 , S. 100-110.

Basso, Andres S, Fernando E Miguez, David A Laird, Robert Horton und Mark Westgate (2013). "Assessing potential of biochar for increasing water-holding capacity of sandy soils". In: $G C B$ Bioenergy 5.2, S. 132-143.

Battaglia, M, ML Cherry, CL Beadle, PJ Sands und A Hingston (1998). "Prediction of leaf area index in eucalypt plantations: effects of water stress and temperature". In: Tree Physiology 18.8-9, S. 521-528.

Bauhus, J (1996). "C and N mineralization in an acid forest soil along a gap-stand gradient". In: Soil Biology and Biochemistry 28.7, S. 923-932. 
Bauters, TWJ, TS Steenhuis, DA DiCarlo, JL Nieber, LW Dekker, CJ Ritsema, J-Y Parlange und R Haverkamp (2000). "Physics of water repellent soils". In: Journal of Hydrology 231, S. 233-243.

BBodSchV (1999). Bundes-Bodenschutz- und Altlastenverordnung.

Beck, W, S Löffler und R Kätzel (2007). "Zum Einfluss von Stickstoffeinträgen auf Wachstum und Vitalität der Kiefer". In: Die Kiefer im nordostdeutschen Tiefland - Ökologie und Bewirtschaftung. Eberswalder Forstliche Schriftenreihe 32, S. 182-198.

Berglund, LM, TH DeLuca und O Zackrisson (2004). "Activated carbon amendments to soil alters nitrification rates in Scots pine forests". In: Soil Biology and Biochemistry 36.12, S. 2067-2073.

Berihun, Tariku, Muluken Tadele und Firew Kebede (2017). "The application of biochar on soil acidity and other physico-chemical properties of soils in southern Ethiopia". In: Journal of Plant Nutrition and Soil Science 180.3, S. 381-388.

BMELV (2009). Waldbericht der Bundesregierung 2009. Bundesministerium füer Ern“ehrung, Landwirtschaft und Verbraucherschutz (BMELV).

Bobbink, Roland und Jan GM Roelofs (1995). "Nitrogen critical loads for natural and seminatural ecosystems: the empirical approach". In: Water, Air, and Soil Pollution 85.4, S. 2413 2418.

Bobbink, Roland, Mike Ashmore, Sabine Braun, Walter Flückiger und Isabel JJ Van den Wyngaert (2003). "Empirical nitrogen critical loads for natural and semi-natural ecosystems: 2002 update". In: Empirical critical loads for nitrogen, S. 43-170.

Bolte, A (1999). Abschätzung von Trockensubstanz-, Kohlenstoff-und Nährelementvorräten der Waldbodenflora: Verfahren, Anwendung und Schätztafeln. Ulmer.

Bolte, A, S Anders und A Roloff (2002). "Schätzmodelle zum oberirdischen Vorrat der Waldbodenflora an Trockensubstanz, Kohlenstoff und Makronährelementen". In: Allg. Forst Jagdztg. 173, S. 57-66.

Bolte, A, T Czajkowski, J Bielefeldt und BS Wolff Heinrichs (2009). "Schätzung der oberirdischen Biomassevorräte des Baum-und Strauchunterwuchses in Wäldern auf der Basis von Vegetationsaufnahmen". In: Forstarchiv 7.5, S. 222-228.

Borchard, N, A Wolf, V Laabs, R Aeckersberg, HW Scherer, A Moeller und W Amelung (2012). "Physical activation of biochar and its meaning for soil fertility and nutrient leaching-a greenhouse experiment". In: Soil Use and Management 28.2, S. 177-184.

Boxman, Andries W und Jan GM Roelofs (1988). "Some effects of nitrate versus ammonium nutrition on the nutrient fluxes in Pinus sylvestris seedlings. Effects of mycorrhizal infection". In: Canadian Journal of Botany 66.6, S. 1091-1097.

Boyd, R, RT Furbank und DJ Read (1986). "Ectomycorrhiza and the water relations of trees [Betula pendula, Paxillus involutus; Pinus sylvestris, Suillus bovinus]". In: Physiological and Genetical Aspects of Mycorrhizae. Aspects physiologiques et genetiques des mycorhizes, Dijon (France), 1-5 Jul 1985. INRA.

Braun, Sabine, Vera FD Thomas, Rebecca Quiring und Walter Flückiger (2010). "Does nitrogen deposition increase forest production? The role of phosphorus". In: Environmental Pollution 158.6, S. 2043-2052.

Bréda, Nathalie, Roland Huc, André Granier und Erwin Dreyer (2006). "Temperate forest trees and stands under severe drought: a review of ecophysiological responses, adaptation processes and long-term consequences". In: Annals of Forest Science 63.6, S. 625-644.

Briggs, Christopher M, Joan Breiner, RC Graham et al. (2005). "Contributions of Pinus Ponderosa charcoal to soil chemical and physical properties". In: The ASACSSA-SSSA International Annual Meetings Salt Lake City, USA. 
Buczko, U, Oliver Bens, H Fischer und RF Hüttl (2002). "Water repellency in sandy luvisols under different forest transformation stages in northeast Germany". In: Geoderma 109.1-2, S. $1-18$.

Bundy, LG und JJ Meisinger (1994). "Nitrogen availability indices". In: Methods of Soil Analysis: Part 2-Microbiological and Biochemical Properties 5, S. 951-984.

Burian, K, W Punz und R Schinninger (1982). "Efficiency of pollutant combinations on plants". In: Tasks for vegetation science.

Burrell, Leigh D, Franz Zehetner, Nicola Rampazzo, Bernhard Wimmer und Gerhard Soja (2016). "Long-term effects of biochar on soil physical properties". In: Geoderma 282, S. 96-102.

Buschmann, Claus, S Bilke, A Butterer, A Enk, T Längle, O Wenzel und HK Lichtenthaler (1999). "Einfluß von Trockenstreß auf die Photosyntheseaktivität verschiedener Ökotypen der Buche (Fagus sylvatica L.) - Unterschiede in der Trockenstressempfindlichkeit". In: Berichtsreihe zum Statusseminar der Baden-Württemberg-Projektträgerschaft "Lebensgrundlage Umwelt und ihre Sicherung"(BW-PLUS), FZKA-BWPLUS 15, Diskussionskreis, S. 1-12.

Cakmak, Ismail (2005). "The role of potassium in alleviating detrimental effects of abiotic stresses in plants". In: Journal of Plant Nutrition and Soil Science 168.4, S. 521-530.

Cambui, Camila Aguetoni, Henrik Svennerstam, Linda Gruffman, Annika Nordin, Ulrika Ganeteg und Torgny Näsholm (2011). "Patterns of plant biomass partitioning depend on nitrogen source". In: PLoS One 6.4.

Carlyle, JC (1986). "Nitrogen cycling in forested eco-systems". In: For. Abstr. Bd. 47, S. 308-336.

Castellini, M, L Giglio, M Niedda, AD Palumbo und D Ventrella (2015). "Impact of biochar addition on the physical and hydraulic properties of a clay soil". In: Soil and Tillage Research 154, S. 1-13.

Chan, KY und Zhihong Xu (2009). "Biochar: nutrient properties and their enhancement". In: Biochar for environmental management: Science and Technology 1, S. 67-84.

Chan, KY, L Van Zwieten, I Meszaros, A Downie und S Joseph (2008). "Agronomic values of greenwaste biochar as a soil amendment". In: Soil Research 45.8, S. 629-634.

Chang, Scott X und Daniel J Robison (2003). "Nondestructive and rapid estimation of hardwood foliar nitrogen status using the SPAD-502 chlorophyll meter". In: Forest Ecology and Management 181.3, S. 331-338.

Cheng, Chih-Hsin, Johannes Lehmann und Mark H Engelhard (2008). "Natural oxidation of black carbon in soils: changes in molecular form and surface charge along a climosequence". In: Geochimica et Cosmochimica Acta 72.6, S. 1598-1610.

Ciais, Philippe, Christopher Sabine, Govindasamy Bala, Laurent Bopp, Victor Brovkin, Josep Canadell, Abha Chhabra, Ruth DeFries, James Galloway, Martin Heimann et al. (2014). "Carbon and other biogeochemical cycles". In: Climate change 2013: the physical science basis. Contribution of Working Group I to the Fifth Assessment Report of the Intergovernmental Panel on Climate Change. Cambridge University Press, S. 465-570.

Clough, ECM, Justin Pearson und GR Stewart (1989). "Nitrate utilization and nitrogen status in English woodland communities". In: Annales des Sciences Forestières. Bd. 46. Supplement. EDP Sciences, S. 669-672.

Clough, Tim J und Leo M Condron (2010). "Biochar and the nitrogen cycle: introduction". In: Journal of environmental quality 39.4, S. 1218-1223.

Clough, Tim J, Leo M Condron, Claudia Kammann und Christoph Müller (2013). "A review of biochar and soil nitrogen dynamics". In: Agronomy 3.2, S. 275-293.

Cochard, Hervé, Damien Lemoine, Thierry Améglio und André Granier (2001). "Mechanisms of xylem recovery from winter embolism in Fagus sylvatica". In: Tree Physiology 21.1, S. 27-33. 
Cochrane, Thomas T und Thomas A Cochrane (2009). "Differences in the way potassium chloride and sucrose solutions effect osmotic potential of significance to stomata aperture modulation". In: Plant Physiology and Biochemistry 47.3, S. 205-209.

Cole, DW, Helga Van Miegroet und NW Foster (1992). "Retention and Loss of N in the IFS Sites and Evaluation of the Relative Importance of Processes". In:

Comeau, Philip G und James P Kimmins (1989). "Above- and below-ground biomass and production of lodgepole pine on sites with differing soil moisture regimes". In: Canadian Journal of Forest Research 19.4, S. 447-454.

Cruiziat, Pierre, Hervé Cochard und Thierry Améglio (2002). "Hydraulic architecture of trees: main concepts and results". In: Annals of forest science 59.7, S. 723-752.

De Witte, LC, NP Rosenstock, S Van Der Linde und S Braun (2017). "Nitrogen deposition changes ectomycorrhizal communities in Swiss beech forests". In: Science of the Total Environment 605, S. 1083-1096.

Dekker, Louis W und Coen J Ritsema (1994). "How water moves in a water repellent sandy soil: 1. Potential and actual water repellency". In: Water Resources Research 30.9, S. 2507-2517.

DeLuca, Tom H, Michael J Gundale, M Derek MacKenzie und Davey L Jones (2015). "Biochar effects on soil nutrient transformations". In: Biochar for environmental management: science, technology and implementation 2, S. 421-454.

Dempster, Daniel N, Davey L Jones und Daniel V Murphy (2012). "Clay and biochar amendments decreased inorganic but not dissolved organic nitrogen leaching in soil". In: Soil Research 50.3, S. 216-221.

Desprez-Loustau, Marie-Laure, Benoit Marçais, Louis-Michel Nageleisen, Dominique Piou und Andrea Vannini (2006). "Interactive effects of drought and pathogens in forest trees". In: Annals of Forest Science 63.6, S. 597-612.

Devereux, Rachel C, Craig J Sturrock und Sacha J Mooney (2012). "The effects of biochar on soil physical properties and winter wheat growth". In: Earth and Environmental Science Transactions of the Royal Society of Edinburgh 103.1, S. 13-18.

Dixon, RK, GM Wright, GT Behrns, RO Teskey und TM Hinckley (1980). "Water deficits and root growth of ectomycorrhizal white oak seedlings". In: Canadian Journal of Forest Research 10.4, S. 545-548.

Doerr, SH, RA Shakesby und RPDm Walsh (2000). "Soil water repellency: its causes, characteristics and hydro-geomorphological significance". In: Earth-Science Reviews 51.1, S. 33-65.

Dore, Mohammed HI (2005). "Climate change and changes in global precipitation patterns: what do we know?" In: Environment international 31.8, S. 1167-1181.

Downie, Adriana, Alan Crosky und Paul Munroe (2009). "Physical properties of biochar". In: Biochar for environmental management: Science and technology, S. 13-32.

Drabkin, Dmitri (2018). "Nutzung von Biokohlesubstraten zur Wiederaufforstung von Windwurfflächen und auf Weihnachtsbaumkulturen". Diss.

EBC (2012). "European Biochar Certificate-guidelines for a sustainable production of biochar". In: Internet.

Eerden, LJM Van der (1982). "Toxicity of ammonia to plants". In: Agriculture and environment 7.3-4, S. 223-235.

Eichhorn, Johannes (1995). Stickstoffsättigung und ihre Auswirkungen auf das Buchenwaldökosystem der Fallstudie Zierenberg. Forschungszentrum Waldökosysteme.

Eichhorn, Johannes, Martin Guericke und Dirk-Roger Eisenhauser (2016). Waldbauliche Klimaanpassung im regionalen Fokus. Sind unsere Wälder fit für den Klimawandel? oekom.

Ellenberg, Heinz und Christoph Leuschner (2010). Vegetation Mitteleuropas mit den Alpen. UTB.

Elmlinger, MW und H Mohr (1992). "Glutamine synthetase in Scots pine seedlings and its control by blue light and light absorbed by phytochrome". In: Planta 188.3, S. 396-402. 
Enquist, Brian J und Karl J Niklas (2002). "Global allocation rules for patterns of biomass partitioning in seed plants". In: Science 295.5559, S. 1517-1520.

Ernst, Wilfried H (1983). "Ökologische Anpassungsstrategien an Bodenfaktoren". In: Plant Biology 96.1, S. 49-71.

Evers, FH (1964). "Die Bedeutung der Stickstofform für Wachstum und Ernährung der Pflanzen, insbesondere der Waldbäume". In: Mitteilungen des Vereins für Forstliche Standortskunde und Forstpflanzenzüchtung 14, S. 19-37.

- (1972). "Die jahrweisen Fluktuationen der Nährelementkonzentrationen in Fichtennadeln und ihre Bedeutung fur die Interpretation nadelanalytischer Befunde". In: Allg. Forst- und Jagdzeitung 143.3/4.

Fangmeier, Andreas, Angelika Hadwiger-Fangmeier, Ludger Van der Eerden und Hans-Jürgen Jäger (1994). "Effects of atmospheric ammonia on vegetation: a review". In: Environmental pollution 86.1 , S. 43-82.

Finér, Leena, H-S Helmisaari, Krista Lõhmus, Hooshang Majdi, Ivano Brunner, Isabella Børja, T Eldhuset, D Godbold, Tine Grebenc, Bohdan Konôpka et al. (2007). "Variation in fine root biomass of three European tree species: Beech (Fagus sylvatica L.), Norway spruce (Picea abies L. Karst.), and Scots pine (Pinus sylvestris L.)" In: Plant Biosystems 141.3, S. 394-405.

Fischer, RA und Theodore C Hsiao (1968). "Stomatal opening in isolated epidermal strips of Vicia faba. II. Responses to $\mathrm{KCl}$ concentration and the role of potassium absorption". In: Plant Physiology 43.12, S. 1953-1958.

Flaig, Holger und Hans Mohr (1992). "Assimilation of nitrate and ammonium by the Scots pine (Pinus sylvestris) seedling under conditions of high nitrogen supply". In: Physiologia Plantarum 84.4, S. 568-576.

Flückiger, W und S Braun (1998). "Nitrogen deposition in Swiss forests and its possible relevance for leaf nutrient status, parasite attacks and soil acidification". In: Nitrogen, the Confer-Ns. Elsevier, S. 69-76.

Flückiger, Walter und Sabine Braun (2003). "Critical limits for nutrient concentrations and ratios for forest trees-a comment". In: Empirical Critical Loads for Nitrogen. B. Achermann and R. Bobbink, ed. Swiss Agency for the Environment, Forests and Landscape, Berne, Switzerland, S. $273-280$.

Galloway, James N, John D Aber, Jan Willem Erisman, Sybil P Seitzinger, Robert W Howarth, Ellis B Cowling und B Jack Cosby (2003). "The nitrogen cascade". In: AIBS Bulletin 53.4, S. 341-356.

Garbaye, Jean und Jean-Louis Churin (1997). "Growth stimulation of young oak plantations inoculated with the ectomycorrhizal fungus Paxillus involutus with special reference to summer drought". In: Forest Ecology and Management 98.3, S. 221-228.

Gaskin, JW, C Steiner, K Harris, KC Das und B Bibens (2008). "Effect of low-temperature pyrolysis conditions on biochar for agricultural use". In: Transactions of the ASABE 51.6, S. 2061-2069.

Gerendás, Jóska, Zhujun Zhu, Roy Bendixen, R George Ratcliffe und Burkhard Sattelmacher (1997). "Physiological and biochemical processes related to ammonium toxicity in higher plants". In: Journal of Plant Nutrition and Soil Science 160.2, S. 239-251.

Gimbel, Katharina F, Heike Puhlmann und Markus Weiler (2016). "Does drought alter hydrological functions in forest soils?" In: Hydrology and Earth System Sciences 20.3, S. 1301-1317.

Givan, Curtis V (1979). "Metabolic detoxification of ammonia in tissues of higher plants". In: Phytochemistry 18.3, S. 375-382.

Glaser, Bruno (1999). Eigenschaften und Stabilität des Humuskörpers derÏndianerschwarzerden ̈̈mazoniens. Lehrstuhl für Bodenkunde und Bodengeographie der Univ. 
Glaser, Bruno, Johannes Lehmann und Wolfgang Zech (2002). "Ameliorating physical and chemical properties of highly weathered soils in the tropics with charcoal-a review". In: Biology and fertility of soils 35.4, S. 219-230.

Göbl, Friederike und Hannes Platzer (1967). Düngung und Mykorrhizabildung bei Zirbenjungpflanzen. Forstliche Bundesversuchsanstalt.

Graber, Ellen R, Yael Meller Harel, Max Kolton, Eddie Cytryn, Avner Silber, Dalia Rav David, Ludmilla Tsechansky, Menahem Borenshtein und Yigal Elad (2010). "Biochar impact on development and productivity of pepper and tomato grown in fertigated soilless media". In: Plant and Soil 337.1-2, S. 481-496.

Granier, A, N Bréda, Ph Biron und S Villette (1999). "A lumped water balance model to evaluate duration and intensity of drought constraints in forest stands". In: Ecological modelling 116.2-3, S. 269-283.

Grennfelt, P und E Thörnelöf (1992). "Critical loads for nitrogen". In: Report from a workshop held at Lökeberg, Sweden. Bd. 6. 10.

Gronwald, Marco, Cora Vos, Mirjam Helfrich und Axel Don (2016). "Stability of pyrochar and hydrochar in agricultural soil-a new field incubation method". In: Geoderma 284, S. 85-92.

Guehl, JM, J Garbaye und A Wartinger (1992). "The effects of ectomycorrhizal status on plantwater relations and sensitivity of leaf-gas exchange to soil drought in Douglas fir (Pseudotsuga menziesii) seedlings". In: Mycorrhizas in ecosystems. CAB International, Wallingford, Oxfordshire, UK, S. 323-332.

Gundale, Michael J und Thomas H DeLuca (2006). "Temperature and source material influence ecological attributes of ponderosa pine and Douglas-fir charcoal". In: Forest Ecology and Management 231.1, S. 86-93.

Hammel, K und M Kennel (2001). "Charakterisierung und Analyse der Wasserverfügbarkeit und des Wasserhaushalts von Waldstandorten in Bayern mit dem Simulationsmodell BROOK90". In: Forstliche Forschungsberichte München 185.

Hammes, K und MWI Schmidt (2009). "Changes of biochar in soil. IN Lehmann J. and Joseph, S.(Eds.) Biochar for environmental management". In: Science and Technology. London, Earthscan.

Hartge, Karl Heinrich, Rainer Horn, Jörg Bachmann und Stephan Peth (2014). Einführung in die Bodenphysik. Schweizerbart'sche Verlagsbuchhandlung.

Haubold-Rosar, Michael, Jürgen Kern und Jürgen Reinhold (2014). Chancen und Risiken des Einsatzes von Biokohle und anderer "veränderter"Biomasse als Bodenhilfsstoffe oder für die C-Sequestrierung in Böden. Forschungsinst. für Bergbaufolgelandschaften.

Heinrichs, Steffi, Markus Bernhardt-Römermann und Wolfgang Schmidt (2010). "The estimation of aboveground biomass and nutrient pools of understorey plants in closed Norway spruce forests and on clearcuts". In: European journal of forest research 129.4, S. 613-624.

Herath, HMSK, Marta Camps-Arbestain und Mike Hedley (2013). "Effect of biochar on soil physical properties in two contrasting soils: an Alfisol and an Andisol". In: Geoderma 209, S. $188-197$.

HessenForst, Landesbetrieb (2014). Forsteinrichtungswerk Staatswald Lampertheim Stichtag 1.1. 2012. Servicezentrum Forsteinrichtung und Naturschutz.

Hock, Bertold (1988). Schadwirkungen auf Pflanzen: Lehrbuch der Pflanzentoxikologie. BI Wissenschaftsverl.

Hofmann, G (2007). "Die Waldkiefer (Pinus sylvestris) in der Vegetation des nordostdeutschen Tieflandes". In: MLUV: Die Kiefer im nordostdeutschen Tiefland-Ökologie und Bewirtschaftung. Eberswalder Forstliche Schriftenreihe, Bd 32, S. 41-53.

Holland, John H (1992). "Complex adaptive systems". In: Daedalus 121.1, S. 17-30. 
Holling, Crawford S (1973). "Resilience and stability of ecological systems". In: Annual review of ecology and systematics 4.1, S. 1-23.

Hoopen, Floor ten, Tracey Ann Cuin, Pai Pedas, Josefine N Hegelund, Sergey Shabala, Jan K Schjoerring und Thomas P Jahn (2010). "Competition between uptake of ammonium and potassium in barley and Arabidopsis roots: molecular mechanisms and physiological consequences". In: Journal of Experimental Botany 61.9, S. 2303-2315.

Ibrom, A und M Runge (1989). "Die Stickstoff-Mineralisation im Boden eines SauerhumusBuchenwaldes unter dem Einfluß von Kalkung oder Stickstoffdüngung". In: Ergebnisse neuerer, ökosystemarer Untersuchungen an Buchenbeständen im Solling. Berichte des Forschungszentrums Waldökosysteme A 49, S. 129-140.

Jacobsen, Carsten und Thomas Ullrich (2010). "Forstökologische Beweissicherung Hessisches Ried-4". In: Zwischenbericht. Landesbetrieb Hessen-Forst.

Jeffery, Simon, Frank GA Verheijen, Marijn van der Velde und Ana Catarina Bastos (2011). "A quantitative review of the effects of biochar application to soils on crop productivity using meta-analysis". In: Agriculture, ecosystems \& environment 144.1, S. 175-187.

Jeffery, Simon, Marcel BJ Meinders, Cathelijne R Stoof, T Martijn Bezemer, Tess FJ van de Voorde, Liesje Mommer und Jan Willem van Groenigen (2015). "Biochar application does not improve the soil hydrological function of a sandy soil". In: Geoderma 251, S. 47-54.

Joseph, SD, Marta Camps-Arbestain, Yun Lin, P Munroe, CH Chia, J Hook, L Van Zwieten, S Kimber, A Cowie, BP Singh et al. (2010). "An investigation into the reactions of biochar in soil". In: Soil Research 48.7, S. 501-515.

Joseph, Stephen, Claudia I Kammann, Jessica G Shepherd, Pellegrino Conte, Hans-Peter Schmidt, Nikolas Hagemann, Anne M Rich, Christopher E Marjo, Jessica Allen, Paul Munroe et al. (2018). "Microstructural and associated chemical changes during the composting of a high temperature biochar: mechanisms for nitrate, phosphate and other nutrient retention and release". In: Science of the Total Environment 618, S. 1210-1223.

Jungk, A2 (1970). "Wechselwirkungen zwischen Stickstoffkonzentration (NH, NHNO und NO) und pH der Nährlösung auf Wuchs und Ionenhaushalt von Tomatenpflanzen". In: Gartenbauwissenschaft, S. 13-28.

Kahle, HP, H Spiecker, PJ Perez-Martinez und R Unseld (2005). "Causes of changes in growth of European Forests: Analysis of the role of climatic factors and nitrogen nutrition". In: The International Forestry Review 7.5, S. 89.

Kameyama, K, T Miyamoto, T Shiono und Y Shinogi (2012). "Influence of sugarcane bagassederived biochar application on nitrate leaching in calcaric dark red soil". In: Journal of Environmental Quality 41.4, S. 1131-1137.

Kammann, Claudia Irene, Sebastian Linsel, Johannes W Gößling und Hans-Werner Koyro (2011). "Influence of biochar on drought tolerance of Chenopodium quinoa Willd and on soil-plant relations". In: Plant and Soil 345.1-2, S. 195-210.

Karhu, Kristiina, Tuomas Mattila, Irina Bergström und Kristiina Regina (2011). "Biochar addition to agricultural soil increased $\mathrm{CH} 4$ uptake and water holding capacity-results from a short-term pilot field study". In: Agriculture, Ecosystems \& Environment 140.1, S. 309-313.

Karl, Thomas R, Richard W Knight und Neil Plummer (1995). "Trends in high-frequency climate variability in the twentieth century". In: Nature 377.6546, S. 217.

Kätzel, R und S Löffler (2007). "Langzeituntersuchungen zu physiologischen Anpassungsreaktionen der Kiefer auf Dauerbeobachtungsflächen der forstlichen Umweltkontrolle (Level-II)". In: Die Kiefer im nordostdeutschen Tiefland-Ökologie und Bewirtschaftung. Eberswalde, Eberswalder Forstliche Schriftenreihe, Bd 32, S. 133-144.

Kätzel, R, S Löffler und K Möller (2008). "Zur physiologischen Anpassungsfähigkeit der Waldkiefer". In: Beiträge der NW-FVA 2, S. 43-61. 
Kätzel, R, M Zander und S Löffler (2015). "Wenn der Durst kommt-Eiche, Buche, Kiefer und Douglasie im Trockenstresstest". In: Eberswalder Forstliche Schriftenreihe, Bd 59, S. 40-54.

Kemper, Tobias, Robert Riechel und Tobias Schuller (2011). Kommunen im Klimawandel-Wege zur Anpassung.

Keyes, Michael R und Charles C Grier (1981). "Above-and below-ground net production in 40year-old Douglas-fir stands on low and high productivity sites". In: Canadian Journal of Forest Research 11.3, S. 599-605.

Kinney, TJ, CA Masiello, B Dugan, WC Hockaday, MR Dean, K Zygourakis und RT Barnes (2012). "Hydrologic properties of biochars produced at different temperatures". In: Biomass and Bioenergy 41, S. 34-43.

Kinzel, Helmut (1982). Pflanzenökologie und Mineralstoffwechsel. Ulmer.

Kjøller, Rasmus, Lars-Ola Nilsson, Karin Hansen, Inger Kappel Schmidt, Lars Vesterdal und Per Gundersen (2012). "Dramatic changes in ectomycorrhizal community composition, root tip abundance and mycelial production along a stand-scale nitrogen deposition gradient". In: New phytologist 194.1, S. 278-286.

Klinck, U und D Fröhlich (2009). "Application of the phytomass and elemental stock model "PhytoCalc" under clear-cut conditions". In: Allg Forst Jagdztg 180, S. 15-21.

Klute, Arnold (1986). "Physical and mineralogical methods". In: Planning 8, S. 79.

Knoepp, Jennifer D und James M Vose (2007). "Regulation of nitrogen mineralization and nitrification in Southern Appalachian ecosystems: separating the relative importance of biotic vs. abiotic controls". In: Pedobiologia 51.2, S. 89-97.

König, N (2009). Handbuch Forstliche Analytik: Eine lose Blattsammlung der Analysemethoden im Forstbereich, S. 568.

Krauß, HH und D Heinsdorf (2005). "Ernährungsstufen für wichtige Wirtschaftsbaumarten". In: Beitr Forstwirtsch Landsch ökol 39, S. 172-179.

Kursar, Thomas A, Bettina MJ Engelbrecht, Amy Burke, Melvin T Tyree, Bouchra EI Omari und Juan P Giraldo (2009). "Tolerance to low leaf water status of tropical tree seedlings is related to drought performance and distribution". In: Functional Ecology 23.1, S. 93-102.

Laird, David A, Pierce Fleming, Dedrick D Davis, Robert Horton, Baiqun Wang und Douglas L Karlen (2010). "Impact of biochar amendments on the quality of a typical Midwestern agricultural soil". In: Geoderma 158.3-4, S. 443-449.

Larcher, Walter (1994). Ökophysiologie der Pflanzen. Eugen Ulmer Stuttgart.

Laubhann, Daniel, Hubert Sterba, Gert Jan Reinds und Wim De Vries (2009). "The impact of atmospheric deposition and climate on forest growth in European monitoring plots: An individual tree growth model". In: Forest Ecology and Management 258.8, S. 1751-1761.

Le Dantec, Valérie, Eric Dufrêne und Bernard Saugier (2000). "Interannual and spatial variation in maximum leaf area index of temperate deciduous stands". In: Forest Ecology and Management 134.1-3, S. 71-81.

LeBauer, David S und Kathleen K Treseder (2008). "Nitrogen limitation of net primary productivity in terrestrial ecosystems is globally distributed". In: Ecology 89.2, S. 371-379.

Lehmann, J und S Joseph (2009). "Biochar for Environmental Management. Earthscan". In: London, UK.

Lehmann, Johannes (2007). "Bio-energy in the black". In: Frontiers in Ecology and the Environment 5.7, S. 381-387.

Lehmann, Johannes, John Gaunt und Marco Rondon (2006). "Bio-char sequestration in terrestrial ecosystems-a review". In: Mitigation and adaptation strategies for global change 11.2, S. $395-419$. 
Lehmann, Johannes, Matthias C Rillig, Janice Thies, Caroline A Masiello, William C Hockaday und David Crowley (2011). "Biochar effects on soil biota-a review". In: Soil biology and biochemistry 43.9, S. 1812-1836.

Lei, Ouyang und Renduo Zhang (2013). "Effects of biochars derived from different feedstocks and pyrolysis temperatures on soil physical and hydraulic properties". In: Journal of Soils and Sediments 13.9, S. 1561-1572.

Lemoine, Damien, Hervé Cochard und André Granier (2002). "Within crown variation in hydraulic architecture in beech (Fagus sylvatica L): evidence for a stomatal control of xylem embolism". In: Annals of forest science 59.1, S. 19-27.

Liang, B, Johannes Lehmann, Dawit Solomon, James Kinyangi, Jonathan Grossman, B O'neill, JO Skjemstad, J Thies, FJ Luizao, J Petersen et al. (2006). "Black carbon increases cation exchange capacity in soils". In: Soil Science Society of America Journal 70.5, S. 1719-1730.

Lu, Sheng-Gao, Fang-Fang Sun und Yu-Tong Zong (2014). "Effect of rice husk biochar and coal fly ash on some physical properties of expansive clayey soil (Vertisol)". In: Catena 114, S. 3744.

Lychuk, Taras E, Roberto C Izaurralde, Robert L Hill, William B McGill und Jimmy R Williams (2015). "Biochar as a global change adaptation: predicting biochar impacts on crop productivity and soil quality for a tropical soil with the Environmental Policy Integrated Climate (EPIC) model". In: Mitigation and Adaptation Strategies for Global Change 20.8, S. 1437-1458.

Lyr, H, H Polster und HJ Fiedler (1967). Gehölzphysiologie.

Lyr, H, HJ Fiedler und W Tranquillini (1992). "Physiologie und Ökologie der Gehölze". In: Fischer, Jena.

Magill, Alison H, John D Aber, Joseph J Hendricks, Richard D Bowden, Jerry M Melillo und Paul A Steudler (1997). "Biogeochemical response of forest ecosystems to simulated chronic nitrogen deposition". In: Ecological applications 7.2, S. 402-415.

Magill, Alison H, John D Aber, William S Currie, Knute J Nadelhoffer, Mary E Martin, William H McDowell, Jerry M Melillo und Paul Steudler (2004). "Ecosystem response to 15 years of chronic nitrogen additions at the Harvard Forest LTER, Massachusetts, USA". In: Forest Ecology and Management 196.1, S. 7-28.

Major, Julie, Johannes Lehmann, Marco Rondon und Christine Goodale (2010). "Fate of soilapplied black carbon: downward migration, leaching and soil respiration". In: Global Change Biology 16.4, S. 1366-1379.

Mao, Jiefei, Klaas GJ Nierop, Jaap S Sinninghe Damsté und Stefan C Dekker (2014). "Roots induce stronger soil water repellency than leaf waxes". In: Geoderma 232, S. 328-340.

Marschner, H (1995). "Mineral nutrition of higher plants.. ed. 2". In:

McMichael, BL und JE Quisenberry (1993). "The impact of the soil environment on the growth of root systems". In: Environmental and experimental botany 33.1, S. 53-61.

McNulty, Steven G und Johnny L Boggs (2010). "A conceptual framework: Redefining forest soil's critical acid loads under a changing climate". In: Environmental Pollution 158.6, S. 2053-2058.

McNulty, Steven G, Johnny L Boggs und Ge Sun (2014). "The rise of the mediocre forest: why chronically stressed trees may better survive extreme episodic climate variability". In: New forests 45.3 , S. 403-415.

Meesenburg, Henning, Agustín Merino, Karl J Meiwes und Friedrich O Beese (2004). "Effects of long-term application of ammonium sulphate on nitrogen fluxes in a beech ecosystem at Solling, Germany". In: Water, Air and Soil Pollution: Focus 4.2-3, S. 415-426.

Meesenburg, Henning, Bernd Ahrends, Stefan Fleck, Markus Wagner, Heike Fortmann, Birte Scheler, Uwe Klinck, Inge Dammann, Johannes Eichhorn und Michael Mindrup (2016). "Longterm changes of ecosystem services at Solling, Germany: Recovery from acidification, but increasing nitrogen saturation?" In: Ecological indicators 65, S. 103-112. 
Mellert, Karl Heinz und Axel Göttlein (2012). "Comparison of new foliar nutrient thresholds derived from van den Burgs literature compilation with established central European references". In: European Journal of Forest Research 131.5, S. 1461-1472.

Mengel, Konrad, Ernest A Kirkby et al. (1978). "Principles of plant nutrition." In: Principles of plant nutrition.

Meyer, FH (1983). Mykologische Beobachtungen zum Baumsterben. Inst. für Landschaftspflege und Naturschutz der Univ. Hannover.

- (1985). "Einfluß des Stickstoff-Faktors auf den Mykorrhizabesatz von Fichtensämlingen im Humus einer Waldschadensfläche". In: AFZ 9.10, S. 208-219.

- (1988). "Ectomycorrhiza and decline of trees". In: Ectomycorrhiza and acid rain. Edited by AE Jansen, J. Dighton, and AHM Bresser. Res. Rep 12, S. 9-31.

Mikola, Peitsa (2012). "Symbiosis in forestry practice". In: Ectomycorrhizae: Their ecology and physiology, S. 383.

Mizuta, Kei, Toshitatsu Matsumoto, Yasuo Hatate, Keiichi Nishihara und Tomoki Nakanishi (2004). "Removal of nitrate-nitrogen from drinking water using bamboo powder charcoal". In: Bioresource technology 95.3, S. 255-257.

Mohr, Hans (1990). "Der anorganische Stickstoff - eine von Natur aus knappe Ressource". In: Der Stickstoff. Springer, S. 10-11.

Mohr, K und U Dämmgen (2013). "Immissionsbelastung durch Ammoniak auf Wälder in der Umgebung von Tierhaltungsanlagen: Teil 2: Wirkungen auf die Vegetation, Dosis-WirkungBeziehungen". In: Landbauforsch 63.2, S. 129-142.

Mölder, Andreas, Markus Bernhardt-Römermann und Wolfgang Schmidt (2008). "Herb-layer diversity in deciduous forests: raised by tree richness or beaten by beech?" In: Forest Ecology and Management 256.3, S. 272-281.

Moschrefi, N (1983). "Ein neues Verfahren der Schlämmanalyse für die Bestimmung der Korngrößenzusammensetzung". In: Mitteilungen der Deutschen Bodenkundlichen Gesellschaft 38, S. $115-118$.

Murach, Dieter und Heinrich Wiedemann (1988). Dynamik und chemische Zusammensetzung der Feinwurzeln von Waldbäumen als Maß für die Gefährdung von Waldökosystemen durch toxische Luftverunreinigungen: Forschungsbericht; BMFT-Förderkennz.: 037304 1. Forschungszentrum Waldökosysteme.

Nadelhoffer, KJ, AE Giblin, GR Shaver und JA Laundre (1991). "Effects of temperature and substrate quality on element mineralization in six arctic soils". In: Ecology 72.1, S. 242-253.

Neitzke, Mechthild (1984). "Der Einfluss von Aluminium auf die Keimung und Keimlingsentwicklung der Buche (Fagus sylvatica L.) unter Berücksichtigung von Wechselwirkungen mit Calcium und Stickstofform". Diss. Universität Münster.

Nilsson, Jan (1988). "Critical loads for sulphur and nitrogen". In: Air pollution and Ecosystems. Springer, S. 85-91.

Novak, Jeffrey M, Isabel Lima, Baoshan Xing, Julia W Gaskin, Christoph Steiner, KC Das, Mohamed Ahmedna, Djaafar Rehrah, Donald W Watts und Warren J Busscher (2009). "Characterization of designer biochar produced at different temperatures and their effects on a loamy sand". In: Annals of Environmental Science 3.1, S. 195-206.

Novak, Jeffrey M, Warren J Busscher, Donald W Watts, James E Amonette, James A Ippolito, Isabel M Lima, Julia Gaskin, KC Das, Christoph Steiner und Mohamed Ahmedna (2012). "Biochars impact on soil-moisture storage in an ultisol and two aridisols". In: Soil Science 177.5, S. $310-320$.

NW-FVA (2013). Waldentwicklungsszenarien für das Hessische Ried: Entscheidungsunterstützung vor dem Hintergrund sich beschleunigt ändernder Wasserhaushalts-und Klimabedingun- 
gen und den Anforderungen aus dem Europäischen Schutzgebietssystem Natura 2000. Bd. 10. Universitätsverlag Göttingen.

NW-FVA, Nordwestdeutsche Forstliche Versuchsanstalt (2017). "Waldzustandsbericht 2017". In: Hessen, Niedersachsen, Sachsen-Anhalt, Schleswig-Holstein. Kassel: Printec Offset.

Otto, Hans-Jürgen (1994). Waldökologie. Ulmer.

Paar, Uwe (1994). Untersuchungen zum Einfluß von Ammonium und Nitrat auf wurzelphysiologische Reaktionsmuster der Buche. Berichte des Forschungszentrums Waldökosysteme A, Bd. 115.

Paar, Uwe, Jan Evers, Inge Dammann, Nils König, Andreas Schulze, Marcus Schmidt, Egbert Schönfelder, Birte Scheler, Thomas Ullrich und Johannes Eichhorn (2016). Waldbodenzustandsbericht für Hessen. Schriftenreihe der NW-FVA.

Paavilainen, E (1967). "The effect of fertilization on the root systems of swamp pine stands". In: Folia For 31, S. 1-9.

Page-Dumroese, Deborah S, Peter R Robichaud, Robert E Brown und Joanne M Tirocke (2015). "Water repellency of two forest soils after biochar addition". In: Transactions of the ASABE 58.2 , S. $335-342$.

Parke, Jennifer L, RG Linderman und Charles H Black (1983). "The role of ectomycorrhizas in drought tolerance of Douglas-fir seedlings". In: New Phytologist 95.1, S. 83-95.

Pearson, John und George R Stewart (1993). "The deposition of atmospheric ammonia and its effects on plants". In: New phytologist 125.2, S. 283-305.

Pena, Rodica und Andrea Polle (2014). "Attributing functions to ectomycorrhizal fungal identities in assemblages for nitrogen acquisition under stress". In: The ISME journal 8.2, S. 321.

Pena, Rodica, Javier Tejedor, Bernd Zeller, Michael Dannenmann und Andrea Polle (2013). "Interspecific temporal and spatial differences in the acquisition of litter-derived nitrogen by ectomycorrhizal fungal assemblages". In: New Phytologist 199.2, S. 520-528.

Pharis, Richard P und Paul J Kramer (1964). "The effects of nitrogen and drought on loblolly pine seedlings". In: Forest Science 10.2, S. 143-150.

Piccolo, A, G Pietramellara und JSC Mbagwu (1996). "Effects of coal derived humic substances on water retention and structural stability of Mediterranean soils". In: Soil Use and Management 12.4, S. 209-213.

Plass, W (1981). "Neue quartärgeologisch-bodenkundliche Erkenntnisse und ihre Auswirkungen auf das Ökosystem Wald". In: Vorträge der Tagungen d. Arb.-Gem. Forstl. Standorts-u. Veg.Kde 8, S. 21-63.

Poorter, Hendrik, Karl J Niklas, Peter B Reich, Jacek Oleksyn, Pieter Poot und Liesje Mommer (2012). "Biomass allocation to leaves, stems and roots: meta-analyses of interspecific variation and environmental control". In: New Phytologist 193.1, S. 30-50.

Prietzel, Jörg, Karl Eugen Rehfuess, Ulrich Stetter und Hans Pretzsch (2008). "Changes of soil chemistry, stand nutrition, and stand growth at two Scots pine (Pinus sylvestris L.) sites in Central Europe during 40 years after fertilization, liming, and lupine introduction". In: European Journal of Forest Research 127.1, S. 43-61.

Prost, Katharina, Nils Borchard, Jan Siemens, Timo Kautz, Jean-Marie Séquaris, Andreas Möller und Wulf Amelung (2013). "Biochar affected by composting with farmyard manure". In: Journal of environmental quality 42.1, S. 164-172.

Rajkovich, Shelby, Akio Enders, Kelly Hanley, Charles Hyland, Andrew R Zimmerman und Johannes Lehmann (2012). "Corn growth and nitrogen nutrition after additions of biochars with varying properties to a temperate soil". In: Biology and Fertility of Soils 48.3, S. 271-284.

Rapp, Christine (1991). Untersuchungen zum Einfluß von Kalkung und Ammoniumsulfat-Düngung auf Feinwurzeln und Ektomykorrhizen eines Buchenaltbestandes im Solling. Berichte des Forschungszentrums Waldökosysteme der Universität Göttingen: Reihe A, Bd. 72. 
Reemtsma, JB (1986). "Der Magnesium-Gehalt von Nadeln niedersächsischer Fichtenbestände und seine Beurteilung". In: Allg. Forst. Jagdztg 157, S. 196-200.

Revell, Kenneth Todd (2011). "The effect of fast pyrolysis biochar made from poultry litter on soil properties and plant growth". Diss. Virginia Tech.

Richards, LA (1948). "Porous plate apparatus for measuring moisture retention and transmission by soil." In: Soil Science 66.2, S. 105-110.

Riek, W und M Renger (1994). "Der Wasserhaushalt der Kiefer als Funktion von Boden- und Klimaparametern in den Berliner Forsten". In: Forstarchiv 65, S. 167-171.

Riley, D und SA Barber (1971). "Effect of ammonium and nitrate fertilization on phosphorus uptake as related to root-induced $\mathrm{pH}$ changes at the root-soil interface". In: Soil Science Society of America Journal 35.2, S. 301-306.

Rillig, Matthias C, Marcel Wagner, Mohamed Salem, Pedro M Antunes, Carmen George, HansGünter Ramke, Maria-Magdalena Titirici und Markus Antonietti (2010). "Material derived from hydrothermal carbonization: effects on plant growth and arbuscular mycorrhiza". In: Applied Soil Ecology 45.3, S. 238-242.

Ritter, Georg und H Tolle (1978). "Stickstoffdüngung in Kiefernbeständen und ihre Wirkung auf Mykorrhizabildung und Fruktifikation der Symbiosepilze". In: Beitrage fur die Forstwirtschaft.

Robichaud, PR, SA Lewis und LE Ashmun (2008). New procedure for sampling infiltration to assess post-fire soil water repellency. Res. Note. Techn. Ber. RMRS-RN-33. Rocky Mountain Station US: Department of Agriculture, Forest Service.

Rockström, Johan, Will Steffen, Kevin Noone, Asa Persson, F Stuart Chapin III, Eric Lambin, Timothy Lenton, Marten Scheffer, Carl Folke, Hans Joachim Schellnhuber et al. (2009). "Planetary boundaries: exploring the safe operating space for humanity". In: Ecology and society 14.2 .

Rogovska, Natalia, David Laird, Richard Cruse, Pierce Fleming, Tim Parkin und David Meek (2011). "Impact of biochar on manure carbon stabilization and greenhouse gas emissions". In: Soil Science Society of America Journal 75.3, S. 871-879.

Roloff, A (1989). "Pflanzen als Bioindikatoren für Umweltbelastungen. I. Prinzipien der Bioindikation und Beispiel Waldbodenvegetation". In: Forstarchiv 60, S. 184-187.

- (2001). Baumkronen: Verständnis und praktische Bedeutung eines komplexen Naturphänomens. Ulmer.

- (2005). "Phänomene der Anpassung und Optimierung bei Bäumen". In: AFZ-DerWald, S. 641645.

Roloff, A und BM Grundmann (2008). "Waldbaumarten und ihre Verwendung im Klimawandel". In: Arch. Forstw 42, S. 97-109.

Rost-Siebert, Klaus (1985). Untersuchungen zur H-und Al-Ionen-Toxizität an Keimpflanzen von Fichte (Picea abies, Karst.) und Buche (Fagus sylvatica, L.) in Lösungskultur. Forschungszentrum Waldökosysteme, Waldsterben.

Rouault, Gaëlle, Jean-Noël Candau, François Lieutier, Louis-Michel Nageleisen, Jean-Claude Martin und Nathalie Warzée (2006). "Effects of drought and heat on forest insect populations in relation to the 2003 drought in Western Europe". In: Annals of Forest Science 63.6, S. 613624.

Sachs, Lothar und Jürgen Hedderich (2006). Angewandte Statistik: Methodensammlung mit $R$. Springer-Verlag.

Salsac, L (1987). "Nitrate and ammonium nutrition in plants". In: Plant Physiol. Biochem. 25, S. 805-812.

Santantonio, D und RK Hermann (1985). "Standing crop, production, and turnover of fine roots on dry, moderate, and wet sites of mature Douglas-fir in western Oregon". In: Annales des Sciences Forestieres. Bd. 42. 2. EDP Sciences, S. 113-142. 
Saxe, Henrik, David S Ellsworth und James Heath (1998). "Tree and forest functioning in an enriched CO2 atmosphere". In: The New Phytologist 139.3, S. 395-436.

Schaap, Martijn, R Wichink Kruit, S Banzhaf, Thomas Scheuschner, Carlijn Hendriks, Richard Kranenburg, A Segers und P Builtjes (2015). "Atmospheric deposition to German natural and semi-natural ecosystems during 2009". In: Report to PINETI II Project (Project No. FKZ 371263240-1), UBA, Dessau. SRU-Sachverständigenrat für Umweltfragen (2015) Stickstoff: Lösungsstrategien für ein drängendes Umweltproblem.

Scheffer, F (2002). "Lehrbuch der Bodenkunde. Scheffer/Schachtschabel. 15. Aufl". In: Spektrum Akademischer Verlag, Heidelberg.

Scherzer, J, W Schaaf und RF Hüttl (1996). "Eignung von FDR-und TDR-Sonden zur Erfassung der Bodenfeuchte in Kippsubstraten mit erhöhter elektrischer Leitfähigkeit". In: Mitteilungen der Deutschen Bodenkundlichen Gesellschaft 80, S. 279-282.

Schmidt-Walter, Paul (2017). brook90r: Run the LWF-Brook90 hydrological model from within $R$. URL: http://github.com/pschmidtwalter/brook90r.

Schütze, Gudrun (2010). Stickstoff-zuviel des Guten?: Überlastung des Stickstoffkreislaufs zum Nutzen von Umwelt und Mensch wirksam reduzieren. Umweltbundesamt.

Siegel, S Castellan und Jr Castellan (1988). "Nonparametric statistics for the behavioural sciences". In: McGraw-Hill, New York.

Sika, MP und AG Hardie (2014). "Effect of pine wood biochar on ammonium nitrate leaching and availability in a South African sandy soil". In: European journal of soil science 65.1, S. 113119.

Sinclair, TR und MM Ludlow (1985). "Who taught plants thermodynamics? The unfulfilled potential of plant water potential". In: Functional Plant Biology 12.3, S. 213-217.

Sitte, Peter, Hubert Ziegler, Friedrich Ehrendorfer und Andreas Bresinsky (2002). Strasburger Lehrbuch der Botanik. Spektrum Verlag, Heidelberg.

Smil, Vaclav (1997). "Global population and the nitrogen cycle". In: Scientific American 277.1, S. $76-81$.

Sohi, SP, Evelyn Krull, E Lopez-Capel und R Bol (2010). "A review of biochar and its use and function in soil". In: Advances in agronomy 105, S. 47-82.

Solberg, Svein, Matthias Dobbertin, Gert Jan Reinds, Holger Lange, Kjell Andreassen, Paloma Garcia Fernandez, Anders Hildingsson und Wim de Vries (2009). "Analyses of the impact of changes in atmospheric deposition and climate on forest growth in European monitoring plots: a stand growth approach". In: Forest Ecology and Management 258.8, S. 1735-1750.

Solomon, Susan, Dahe Qin, Martin Manning, Kristen Averyt und Melinda Marquis (2007). Climate change 2007-the physical science basis: Working group I contribution to the fourth assessment report of the IPCC. Bd. 4. Cambridge university press.

Sombroek, Wilhelmus Gerardus (1966). Amazon soils. Landbouwhogeschool.

Sombroek, Wim, M de L Ruivo, Philip M Fearnside, Bruno Glaser und Johannes Lehmann (2003). "Amazonian Dark Earths as carbon stores and sinks". In: Amazonian dark earths: origin, properties, management, S. 125-139.

Spokas, Kurt A, Jeff M Novak und Rodney T Venterea (2012). "Biochars role as an alternative N-fertilizer: ammonia capture". In: Plant and Soil 350.1-2, S. 35-42.

Standortskartierung, Arbeitskreis (2003). Forstliche Standortsaufnahme: Begriffe, Definitionen, Einteilungen, Kennzeichnungen, Erläuterungen. Arbeitsgemeinschaft Forsteinrichtung. IHWVerlag, Eching bei München.

Stanford, George und SJ Smith (1972). "Nitrogen mineralization potentials of soils". In: Soil Science Society of America Journal 36.3, S. 465-472. 
Stefan, K, R Fürst, U Hacker und U Bartels (1997). Forest Foliar Condition in Europe. Results of large-scale foliar chemistry surveys. Austrian Federal Forest Research Centre, Brussels, Geneva, Vienna.

Steubing, L und A Fangmeier (1992). Pflanzenökologischen Praktikum: Gelände-und Laborpraktikum der terrestrischen Pflanzenökologie. Ulmer.

Sutmöller, Johannes, Henning Meesenburg, Jan Evers und Markus Wagner (2017). "Auswirkungen der Trockenheit 2015 auf den Bodenwasserhaushalt und das Baumwachstum von Waldstandorten in Nordwestdeutschland". In: Waldböden: Nutzung und Schutz, S. 83.

Täumer, K, H Stoffregen und G Wessolek (2005). "Determination of repellency distribution using soil organic matter and water content". In: Geoderma 125.1-2, S. 107-115.

Topp, G Clarke, JL Davis und Aa P Annan (1980). "Electromagnetic determination of soil water content: Measurements in coaxial transmission lines". In: Water resources research 16.3 , S. 574-582.

Tyree, MT und FW Ewers (1991). "The hydraulic architecture of trees and other woody plants". In: New Phytologist 119.3, S. 345-360.

Tyree, MT, H Cochard, P Cruiziat, B Sinclair und T Ameglio (1993). "Drought-induced leaf shedding in walnut: evidence for vulnerability segmentation". In: Plant, Cell $\&$ Environment 16.7, S. 879-882.

Ulrich, B (1980). "Die Wälder in Mitteleuropa: Messergebnisse ihrer Umweltbelastung, Theorie ihrer Gefährdung, Prognose ihrer Entwicklung". In: Allg. Forstz 35.1, S. 198-2202.

Ulrich, Bernhard (1981). "Ökologische Gruppierung von Böden nach ihrem chemischen Bodenzustand". In: Journal of Plant Nutrition and Soil Science 144.3, S. 289-305.

Unold, G. v. (2000). Empfehlungen für die Gewinnung von Bodenwasser mittels Saugkerzen oder keramischen Platten. UMS GmbH, S. 12.

Van Diest, A (1989). "Eintrag von NO3-und NH4+ in niederländische Waldbestände und die Auswirkungen auf die N-Ernährung und den Vitalitätszustand." In: Kali-Briefe 19, S. 391401.

Van Miegroet, Helga, DW Cole und NW Foster (1992). "Nitrogen distribution and cycling". In: Verheijen, F, S Jeffery, AC Bastos, M Van der Velde und I Diafas (2010). "Biochar application to soils". In: A critical scientific review of effects on soil properties, processes, and functions. EUR 24099, S. 162.

Vines, Herbert Max und RT Wedding (1960). "Some effects of ammonia on plant metabolism and a possible mechanism for ammonia toxicity". In: Plant Physiology 35.6, S. 820.

Vitousek, Peter M, James R Gosz, Charles C Grier, Jerry M Melillo und William A Reiners (1982). "A comparative analysis of potential nitrification and nitrate mobility in forest ecosystems". In: Ecological monographs 52.2, S. 155-177.

Viviani, Gaspare und Massimo Iovino (2004). "Wastewater reuse effects on soil hydraulic conductivity". In: Journal of Irrigation and Drainage Engineering 130.6, S. 476-484.

Vogt, KA, EE Moore, DJ Vogt, MJ Redlin und RL Edmonds (1983). "Conifer fine root and mycorrhizal root biomass within the forest floors of Douglas-fir stands of different ages and site productivities". In: Canadian Journal of Forest Research 13.3, S. 429-437.

Vogt, KA, DJ Vogt, EE Moore, W Littke, CC Grier und L Leney (1985). "Estimating Douglas-fir fine root biomass and production from living bark and starch". In: Canadian journal of forest research 1 , S. 177-179.

Vogt, KA, CC Grier und DJ Vogt (1986). "Production, turnover, and nutrient dynamics of above-and belowground detritus of world forests". In: Advances in ecological research. Bd. 15. Elsevier, S. 303-377. 
Vollbrecht, P, E Klein und H Kasemir (1989). "Different effects of supplied ammonium on glutamine synthetase activity in mustard (Sinapis alba) and pine (Pinus sylvestris) seedlings". In: Physiologia Plantarum 77.1, S. 129-135.

Walker, Brian und David Salt (2012). Resilience thinking: sustaining ecosystems and people in a changing world. Island Press.

Wallis, MG, DR Scotter und DJ Horne (1991). "An evaluation of the intrinsic sorptivity water repellency index on a range of New Zealand soils". In: Soil Research 29.3, S. 353-362.

Wang, Tao, Marta Camps Arbestain, Mike Hedley und Peter Bishop (2012). "Chemical and bioassay characterisation of nitrogen availability in biochar produced from dairy manure and biosolids". In: Organic Geochemistry 51, S. 45-54.

Wardle, David A, Marie-Charlotte Nilsson und Olle Zackrisson (2008). "Fire-derived charcoal causes loss of forest humus". In: Science 320.5876, S. 629-629.

Warnock, Daniel D, Daniel L Mummey, Brooke McBride, Julie Major, Johannes Lehmann und Matthias C Rillig (2010). "Influences of non-herbaceous biochar on arbuscular mycorrhizal fungal abundances in roots and soils: results from growth-chamber and field experiments". In: Applied Soil Ecology 46.3, S. 450-456.

Wessolek, G, WH Duijnisveld und S Trinks (2009). "Hydro-Pedotransferfunktionen zur Berechnung der Sickerwasserrate aus dem Boden-das TUB-BGR-Verfahren". In: Bodenphysikalische Kennwerte und Berechnungsverfahren für die Praxis. Bodenökologie und Bodengenese, Bd 40, S. $66-80$.

Wild, Alan (1995). Umweltorientierte Bodenkunde: eine Einführung. Spektrum Akad. Verlag, Heidelberg.

Wilpert, Klaus v, Bernhard Bösch, Peter Bastian, Dietmar Zirlewagen, Frieder Hepperle, Stefan Holzmann, Heike Puhlmann, Jürgen Schäffer, Gerald Kändler und Udo Hans Sauter (2011). Biomasse-Aufkommensprognose und Kreislaufkonzept für den Einsatz von Holzaschen in der Bodenschutzkalkung in Oberschwaben. Berichte Freiburger Forstliche Forschung FVA-Freiburg, Heft 87.

Wöllecke, J, B Münzenberger und RF Hüttl (1999). "Some effects of N on ectomycorrhizal diversity of Scots pine (Pinus sylvestris L.) in northeastern Germany". In: Water, Air, and Soil Pollution 116.1-2, S. 135-140.

Worzyk, Florian, Rene Schatten, Christian Krüger, Konstantin Terytze und Ines Vogel (2014). "Auswirkungen von Biokohle-Substraten und Biokohle auf Bodenparameter und Pflanzenwachstum MKW-und PAK-kontaminierter Böden". In: Altlasten spektrum 3.2014, S. 101-113.

Wulf, S, A Brenner, J Clemens, H Döhler, P Jäger, KH Krohmer, M Maeting, C Rieger, I Schumacher, M Tscheppe et al. (2003). "Untersuchung der Emission direkt und indirekt klimawirksamer Spurengase während der Lagerung und nach der Ausbringung von Kofermentationsrückständen sowie Entwicklung von Verminderungsstrategien". In: Abschlussbericht DBU-AZ 8912.

Yao, Ying, Bin Gao, Ming Zhang, Mandu Inyang und Andrew R Zimmerman (2012). "Effect of biochar amendment on sorption and leaching of nitrate, ammonium, and phosphate in a sandy soil". In: Chemosphere 89.11, S. 1467-1471.

Zhu, Xiangdong, Yuchen Liu, Feng Qian, Shicheng Zhang und Jianmin Chen (2015). "Investigation on the physical and chemical properties of hydrochar and its derived pyrolysis char for their potential application: influence of hydrothermal carbonization conditions". In: Energy 86 Fuels 29.8, S. 5222-5230.

Zöttl, H von (1964). "Düngung und Feinwurzelverteilung in Fichtenbeständen". In: Mitt. Bayer. Staatsforstverw 34, S. 333-342. 


\section{A Anhang}

\section{Klima und Witterung}
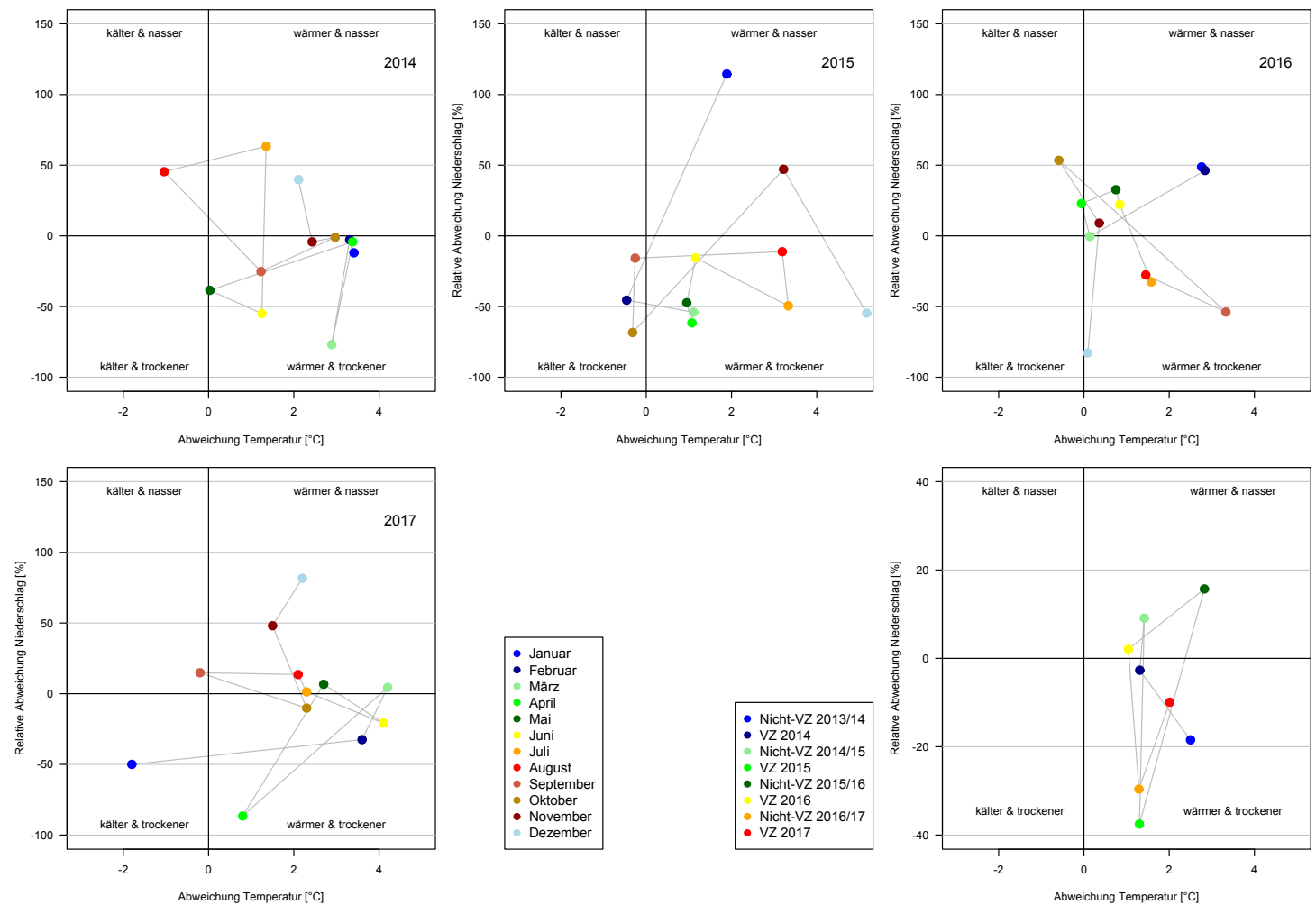

Abbildung A.1: Thermopluviogramme der Monate und Vegetations- und Nichtvegetationszeiten (VZ) der Jahre 2014 bis 2017. Relative Temperatur- und Niederschlagsabweichungen im Vergleich zur Referenzperiode 1961 - 1990. Vegetationszeit 1. April bis 31. Oktober. Datenquelle Deutscher Wetterdienst, Station Mannheim, 2018. 


\section{Bodenphysik}

Tabelle A.1: Korngrößenverteilung [\%] im Ober- $(0-20 \mathrm{~cm})$ und Unterboden $(20-60 \mathrm{~cm})$

\begin{tabular}{cccccc}
\hline Variante & Bodentiefe $[\mathrm{cm}]$ & Ton & Schluff & Sand & Bodenart \\
\hline Null & $0-20$ & 3,9 & 2,9 & 93,2 & $\mathrm{mSfs}$ \\
HKpur & $0-20$ & 3,8 & 2,5 & 93,7 & $\mathrm{mS}$ \\
SKpur & $0-20$ & 4,9 & 2,1 & 93 & $\mathrm{mSfs}$ \\
HK+N+Ko & $0-20$ & 6,4 & 5,6 & 88 & $\mathrm{St} 2$ \\
Null & $20-60$ & 2,5 & 0,9 & $\mathrm{~s} 96,6$ & $\mathrm{mSfs}$ \\
HKpur & $20-60$ & 2,5 & 1,2 & 96,3 & $\mathrm{mSfs}$ \\
SKpur & $20-60$ & 2,9 & 0,9 & 96,2 & $\mathrm{mSfs}$ \\
HK+N+Ko & $20-60$ & 3,7 & 1,8 & 94,5 & $\mathrm{mSfs}$ \\
\hline
\end{tabular}
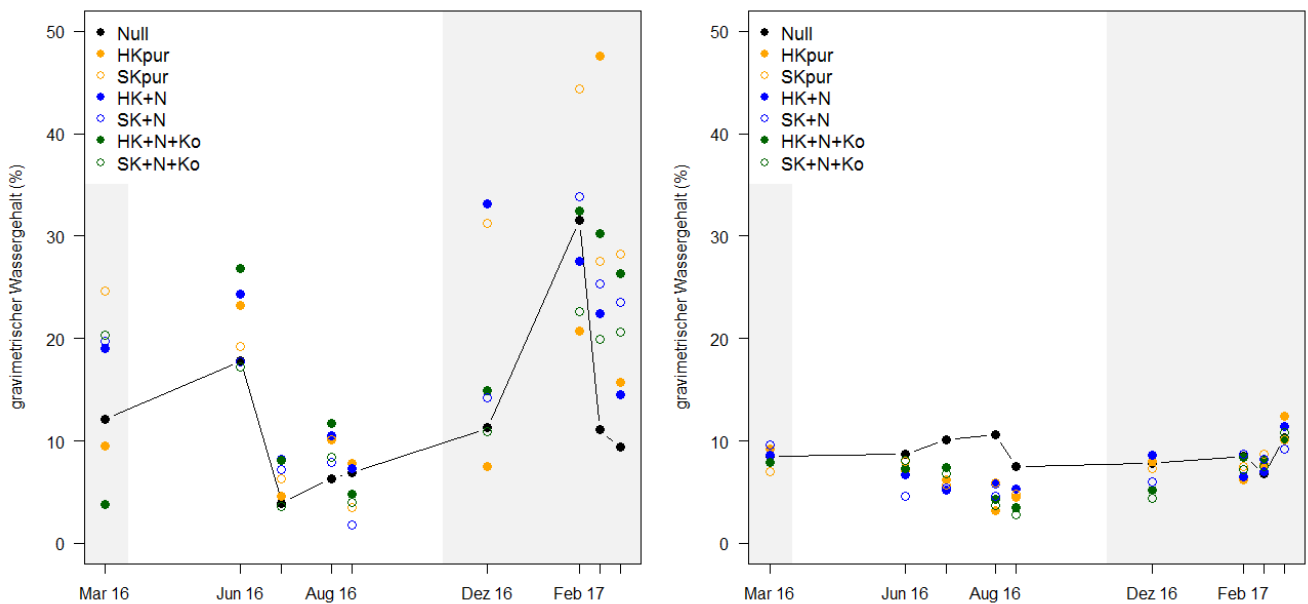

Abbildung A.2: Gravimetrischer Wassergehalt in Ober- $(20-30 \mathrm{~cm}$ Bodentiefe, links) und Unterboden (80 - $90 \mathrm{~cm}$ Bodentiefe, rechts) zwischen März 2016 bis März 2017.

\section{Bodenchemie}

Tabelle A.2: Elementgehalte der Nullflächen vor Kohleeinarbeitung (Standardabweichung)

\begin{tabular}{|c|c|c|c|c|c|c|c|}
\hline Parzelle & $\begin{array}{l}\text { Bodentiefe } \\
\mathrm{cm}\end{array}$ & $\begin{array}{l}\mathrm{C} \\
\mathrm{g} \mathrm{kg}^{-1}\end{array}$ & $\begin{array}{l}\mathrm{N} \\
\mathrm{g} \mathrm{kg}^{-1}\end{array}$ & $\begin{array}{l}\mathrm{Ca} \\
\mathrm{mg} \mathrm{kg}{ }^{-1}\end{array}$ & $\begin{array}{l}\mathrm{Mg} \\
\mathrm{mg} \mathrm{kg}{ }^{-1}\end{array}$ & $\begin{array}{l}\mathrm{K} \\
\mathrm{mg} \mathrm{kg}-1\end{array}$ & $\mathrm{pH}_{\mathrm{H}_{2} \mathrm{O}}$ \\
\hline & $0-30$ & $17,2(4,1)$ & $0,9(0,2)$ & $882,3(134,6)$ & $25,4(7,9)$ & $37,0(8,2)$ & $6,4(0,5)$ \\
\hline & $30-60$ & $1,7(0,6)$ & $0,1(0,04)$ & $638,9(88,5)$ & $7,4(1,0)$ & $20,3(1,7)$ & $8,3(0,4)$ \\
\hline & $60-90$ & $1,0(0,2)$ & $0,1(0,01)$ & $573,3(47,8)$ & $6,6(1,1)$ & $20,2(2,7)$ & $8,7(0,1)$ \\
\hline $1 / 7$ & $0-30$ & 15,5 & 0,8 & 204,2 & 20,2 & 22,8 & 4,6 \\
\hline $1 / 7$ & $30-60$ & 1,8 & 0,1 & 23,5 & 5,1 & 17,2 & 4,7 \\
\hline $1 / 7$ & $60-90$ & 1,5 & 0,1 & 20 & 4,2 & 33,3 & 4,6 \\
\hline
\end{tabular}


Tabelle A.3: Stickstoffgehalte in den Jahren 2014 und 2015. Mittelwert (Standardabweichung)

\begin{tabular}{|c|c|c|c|}
\hline \multirow{2}{*}{ Variante } & \multirow{2}{*}{$\begin{array}{c}\text { Bodentiefe } \\
\mathrm{cm}\end{array}$} & \multicolumn{2}{|c|}{$\mathrm{N}_{\text {ges }}\left[\mathrm{g} \mathrm{kg}^{-1}\right]$} \\
\hline & & 2014 & 2015 \\
\hline Null & $0-30$ & $0,86(0,18)$ & $1,02(0,539)$ \\
\hline HKpur & $0-30$ & & $1,06(0,389)$ \\
\hline SKpur & $0-30$ & & $1,102(0,202)$ \\
\hline $\mathrm{HK}+\mathrm{N}$ & $0-30$ & & $1,013(0,373)$ \\
\hline $\mathrm{SK}+\mathrm{N}$ & $0-30$ & & $1,16(0,238)$ \\
\hline $\mathrm{HK}+\mathrm{N}+\mathrm{Ko}$ & $0-30$ & & $1,403(0,548)$ \\
\hline $\mathrm{SK}+\mathrm{N}+\mathrm{Ko}$ & $0-30$ & & $1,435(0,556)$ \\
\hline Null & $30-60$ & $0,1(0,04)$ & $0,13(0,05)$ \\
\hline HKpur & $30-60$ & & $0,27(0,14)$ \\
\hline SKpur & $30-60$ & & $0,19(0,09)$ \\
\hline $\mathrm{HK}+\mathrm{N}$ & $30-60$ & & $0,28(0,18)$ \\
\hline $\mathrm{SK}+\mathrm{N}$ & $30-60$ & & $0,29(0,07)$ \\
\hline $\mathrm{HK}+\mathrm{N}+\mathrm{Ko}$ & $30-60$ & & $0,3(0,17)$ \\
\hline $\mathrm{SK}+\mathrm{N}+\mathrm{Ko}$ & $30-60$ & & $0,23(0,05)$ \\
\hline Null & $60-90$ & $0,05(0,01)$ & 0,09 \\
\hline HKpur & $60-90$ & & 0,09 \\
\hline SKpur & $60-90$ & & 0,14 \\
\hline $\mathrm{HK}+\mathrm{N}$ & $60-90$ & & 0,12 \\
\hline $\mathrm{SK}+\mathrm{N}$ & $60-90$ & & 0,09 \\
\hline $\mathrm{HK}+\mathrm{N}+\mathrm{Ko}$ & $60-90$ & & 0,14 \\
\hline $\mathrm{SK}+\mathrm{N}+\mathrm{Ko}$ & $60-90$ & & 0,26 \\
\hline
\end{tabular}




\section{Parametrisierung des Sickerwassermodells}

Tabelle A.4: Parameterwerte in Brook90. Nicht aufgeführte Parameter ensprechen Standardwert

\begin{tabular}{ccccc}
\hline Name & Einheit & Null & HKpur & HK+N+Ko \\
\hline MakeStand & & \multicolumn{3}{c}{$2013-2017$} \\
stand.years & & \multicolumn{4}{c}{5} \\
age & doy & \multicolumn{3}{c}{265} \\
budburst.doy & doy & \multicolumn{2}{c}{1} \\
leaffall.doy & & 4 & 5 & 5 \\
sai & & 3 & 4 & 4 \\
maxlai & & 0.7 & 0.9 & 1 \\
minlai & $\mathrm{m}$ & 0.7 & 0.9 & 1 \\
height & & &
\end{tabular}

\begin{tabular}{|c|c|c|c|c|}
\hline \multicolumn{5}{|l|}{ MakeParam.B90 } \\
\hline optdoy & doy & & & \\
\hline $\operatorname{glmax}$ & $\mathrm{m} \mathrm{s}^{-1}$ & & & \\
\hline maxlai & & 4 & 5 & 5 \\
\hline height & $\mathrm{m}$ & 0.7 & 0.9 & 1 \\
\hline densef & & 0.7 & 0.9 & 1 \\
\hline winlaifrac & & 0.9 & 0.8 & 0.8 \\
\hline maxrootdepth & $\mathrm{cm}$ & 70 & 50 & 50 \\
\hline betaroot & & 0.95 & 0.95 & 0.99 \\
\hline inicontrol & & & & \\
\hline rootmodel & & & beta & \\
\hline budburst & & & dyn & \\
\hline leaffall & & & dyn & \\
\hline budburst.method & & & & \\
\hline species_dynbudburst & & & Fagus & \\
\hline humusroots & & & FA & \\
\hline
\end{tabular}




\section{SPAD-Wert und Blatt/Nadel-Elementgehalte}

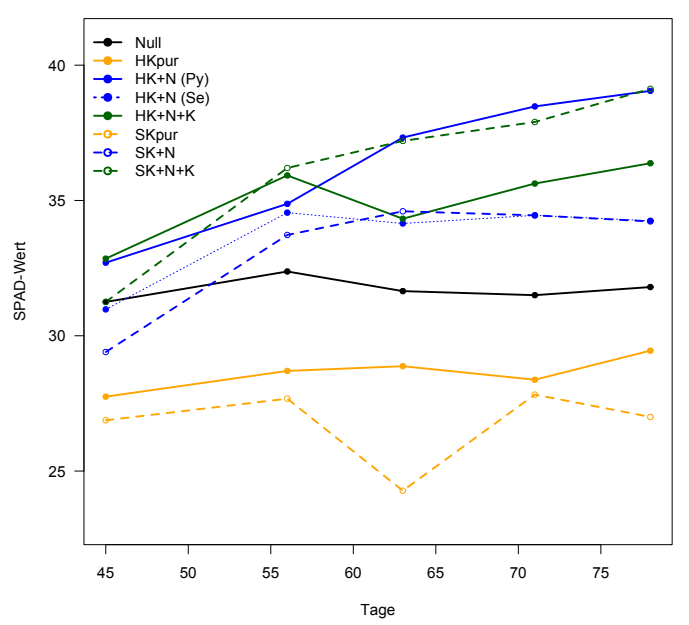

Abbildung A.3: SPAD-Wert der Hainbuchenblätter im Topfversuch.

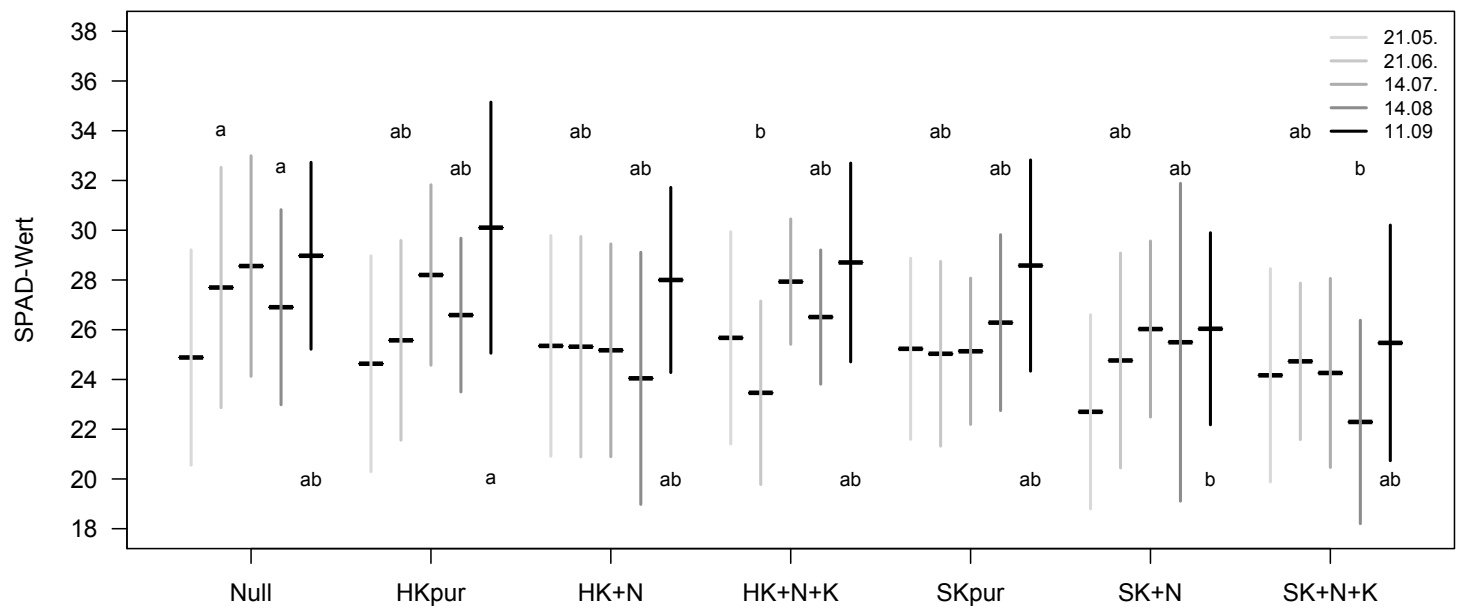

Abbildung A.4: SPAD-Wert der Hainbuchenblätter während der Vegetationszeit 2015. Mittelwerte mit Standardabweichungen des Mittelwertes. Unterschiedliche Buchstaben bezeichnen signifikante Unterschiede an einem Messtermin (Signifikanzniveau $\mathrm{p}<0,05)$. 


\section{Blatt-/Nadel-Elementgehalte von Hainbuche und Kiefer}
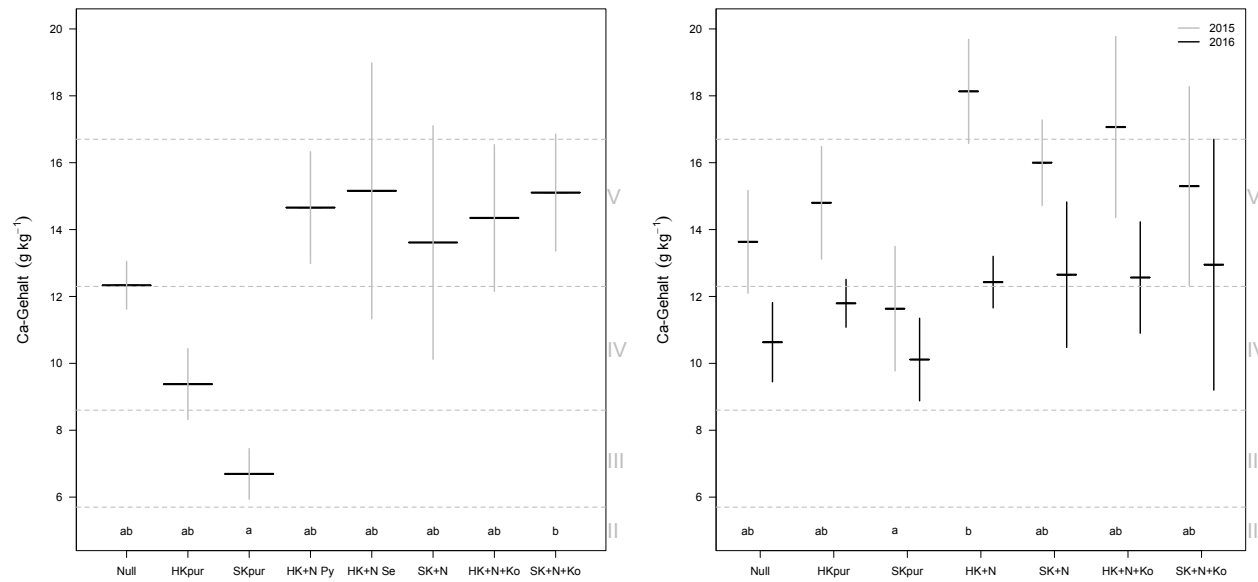

Abbildung A.5: Calciumgehalte der Hainbuchenblätter im Topfversuch (links) und Feldversuch (rechts). Waagerechte gestrichelte Linien kennzeichnen die Grenzen der Ernährungsstufen nach Krauß und Heinsdorf (2005). Mittelwerte mit Standardabweichungen des Mittelwertes. Unterschiedliche Buchstaben bezeichnen signifikante Unterschiede an einem Messtermin (Signifikanzniveau $\mathrm{p}<0,05, \mathrm{n}=3$ ).
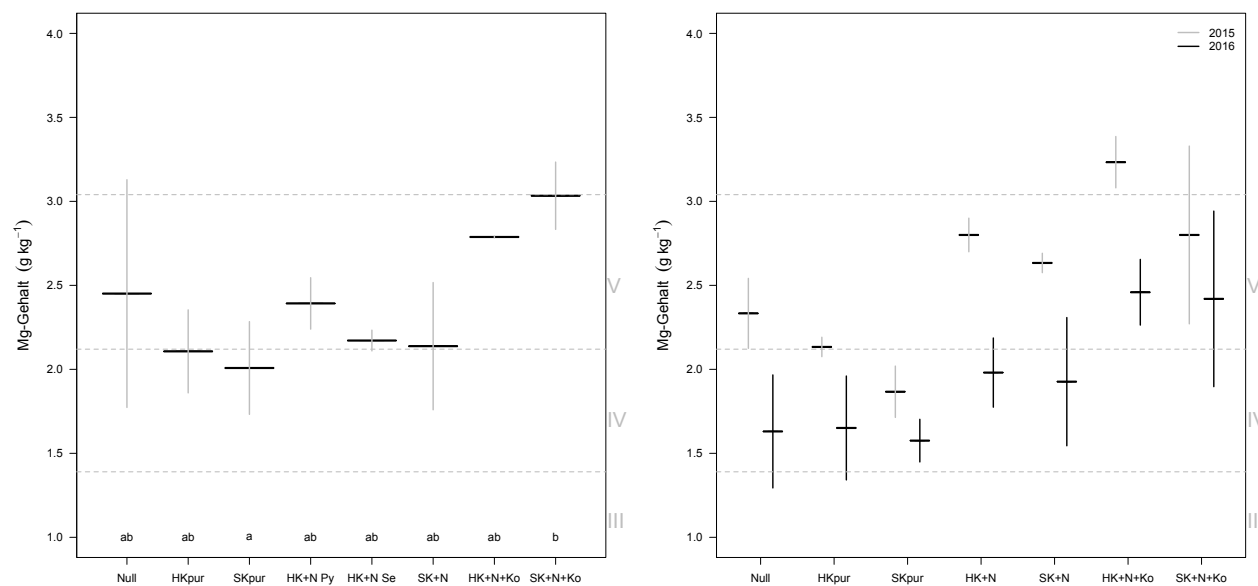

Abbildung A.6: Magnesiumgehalte der Hainbuchenblätter im Topfversuch (links) und Feldversuch (rechts). Waagerechte gestrichelte Linien kennzeichnen die Grenzen der Ernährungsstufen nach Krauß und Heinsdorf (2005). Mittelwerte mit Standardabweichungen des Mittelwertes. Unterschiedliche Buchstaben bezeichnen signifikante Unterschiede an einem Messtermin (Signifikanzniveau $\mathrm{p}<0,05, \mathrm{n}=3$ ). 

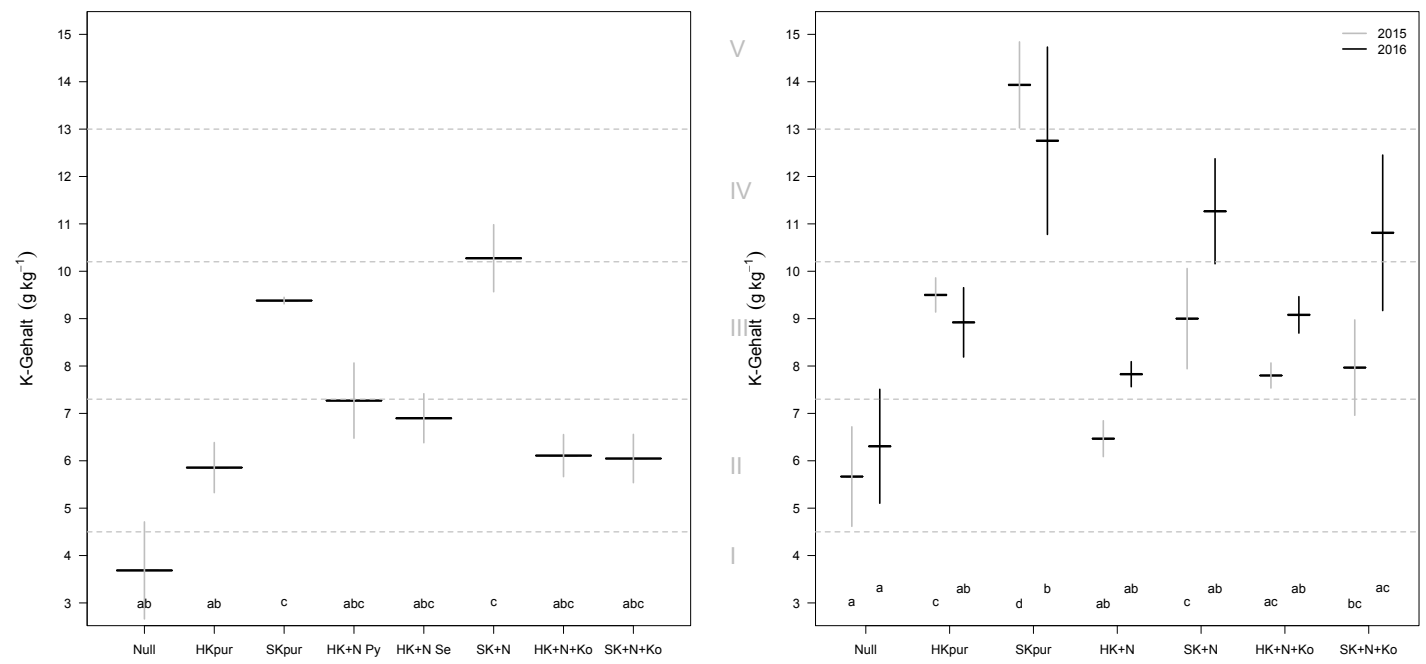

Abbildung A.7: Kaliumgehalte der Hainbuchenblätter im Topfversuch (links) und Feldversuch (rechts). Waagerechte gestrichelte Linien kennzeichnen die Grenzen der Ernährungsstufen nach Krauß und Heinsdorf (2005). Mittelwerte mit Standardabweichungen des Mittelwertes. Unterschiedliche Buchstaben bezeichnen signifikante Unterschiede an einem Messtermin (Signifikanzniveau $\mathrm{p}<0,05, \mathrm{n}=3$ ).
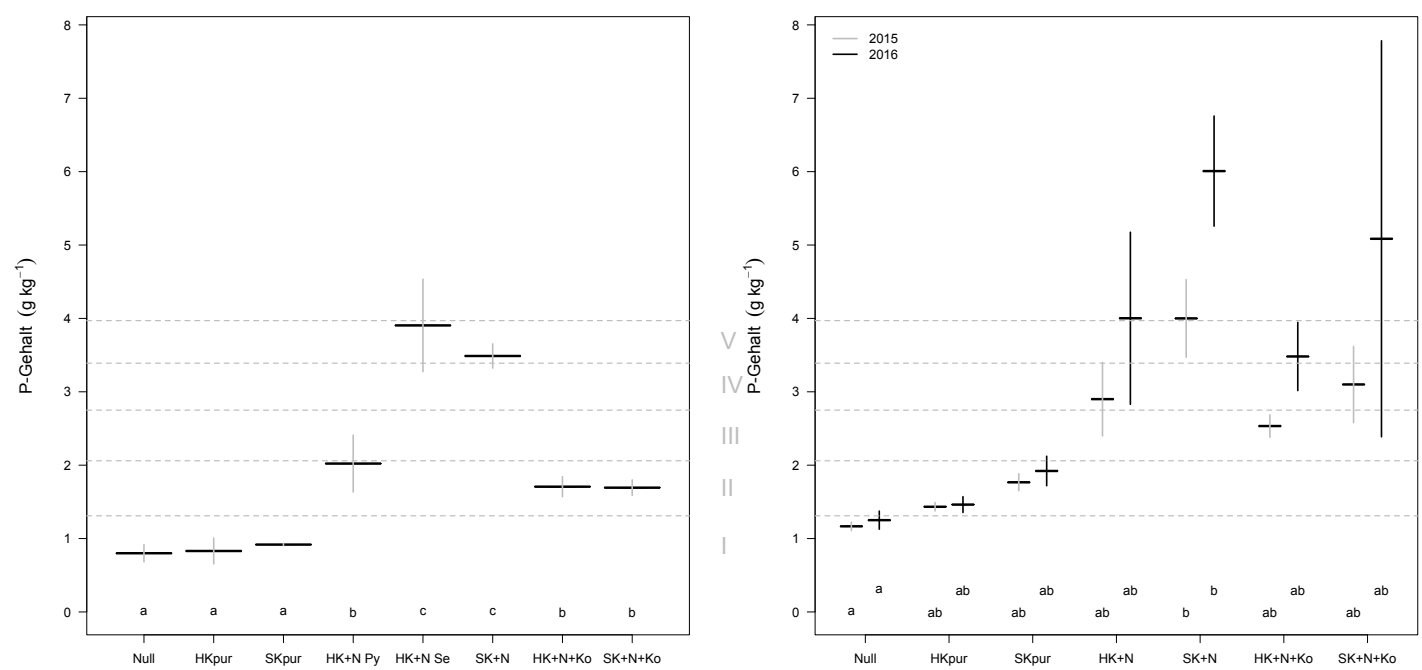

Abbildung A.8: Phosphorgehalte der Hainbuchenblätter im Topfversuch (links) und Feldversuch (rechts). Waagerechte gestrichelte Linien kennzeichnen die Grenzen der Ernährungsstufen nach Krauß und Heinsdorf (2005). Mittelwerte mit Standardabweichungen des Mittelwertes. Unterschiedliche Buchstaben bezeichnen signifikante Unterschiede an einem Messtermin (Signifikanzniveau $\mathrm{p}<0,05, \mathrm{n}=3$ ). 

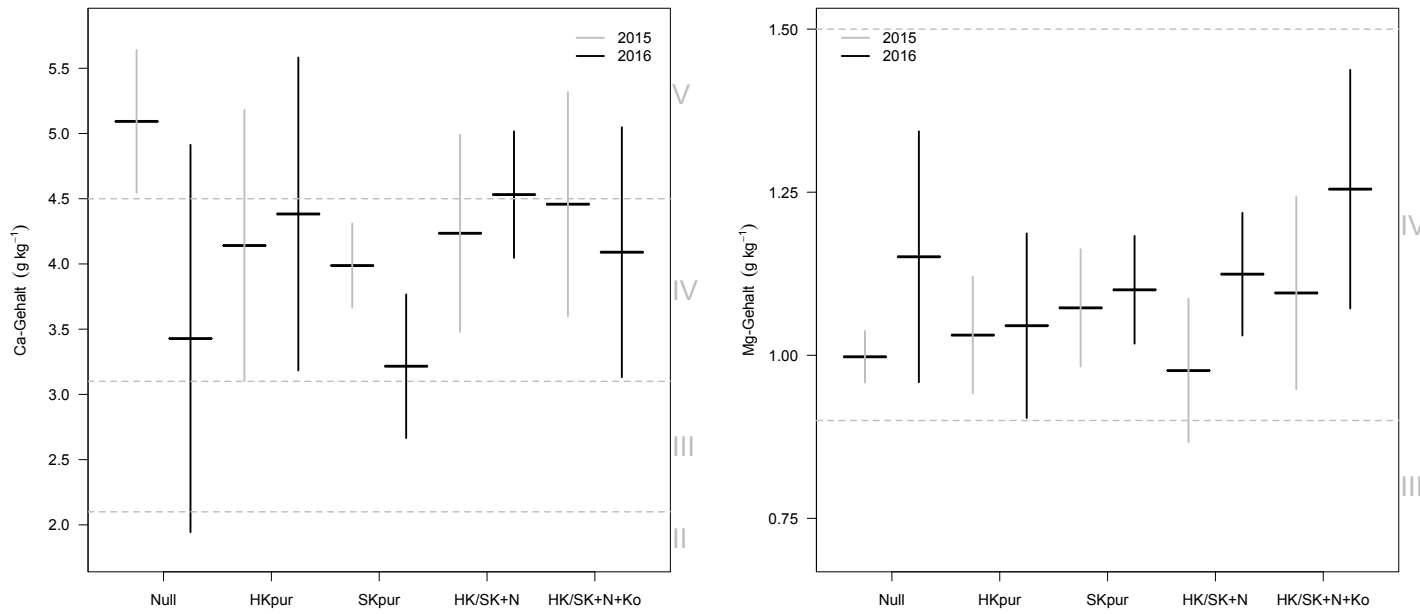

Abbildung A.9: Calcium- (links) und Magnesiumgehalte (rechts) der Kiefernnadeln im Feldversuch in den Jahren 2015 und 2016. Waagerechte gestrichelte Linien kennzeichnen die Grenzen der Ernährungsstufen nach Krauß und Heinsdorf (2005). Mittelwerte mit Standardabweichungen des Mittelwertes, $n=3$ ).
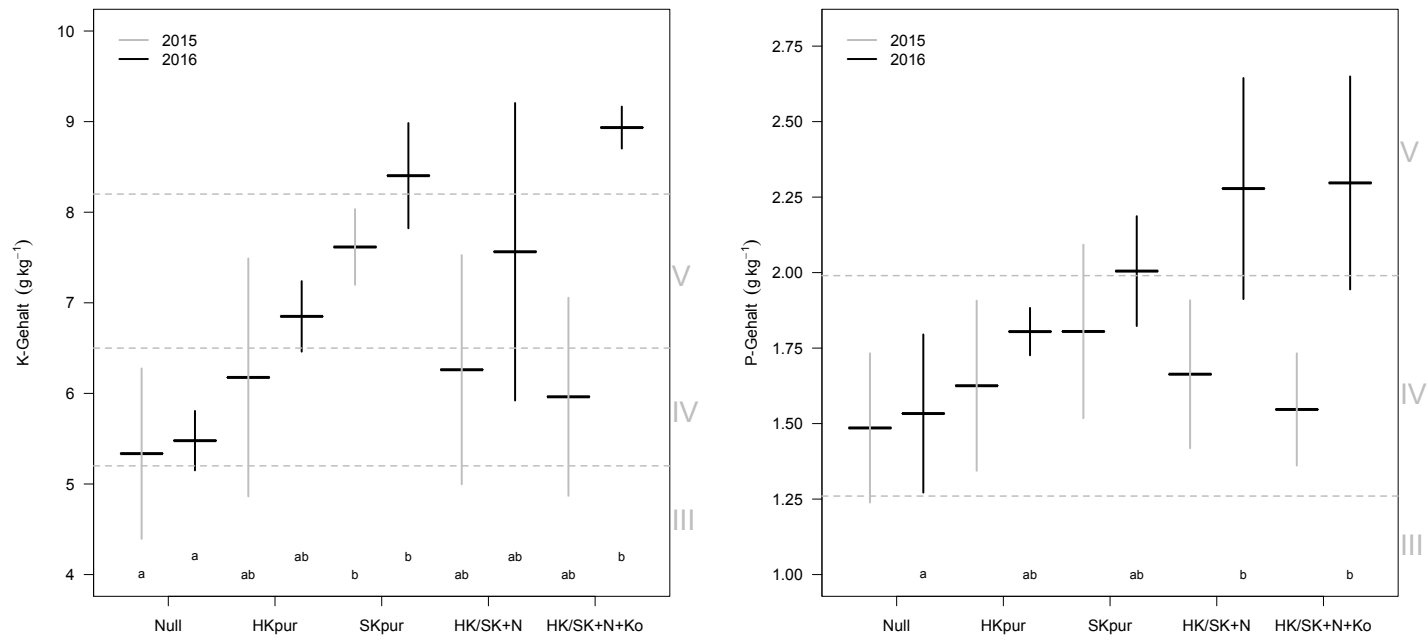

Abbildung A.10: Kalium- (links) und Phosphorgehalte (rechts) der Kiefernnadeln in den Jahren 2015 und 2016. Waagerechte gestrichelte Linien kennzeichnen die Grenzen der Ernährungsstufen nach Krauß und Heinsdorf (2005). Mittelwerte mit Standardabweichungen des Mittelwertes. Unterschiedliche Buchstaben bezeichnen signifikante Unterschiede an einem Messtermin (Signifikanzniveau $\mathrm{p}<0,05$; $\mathrm{n}=3)$. 


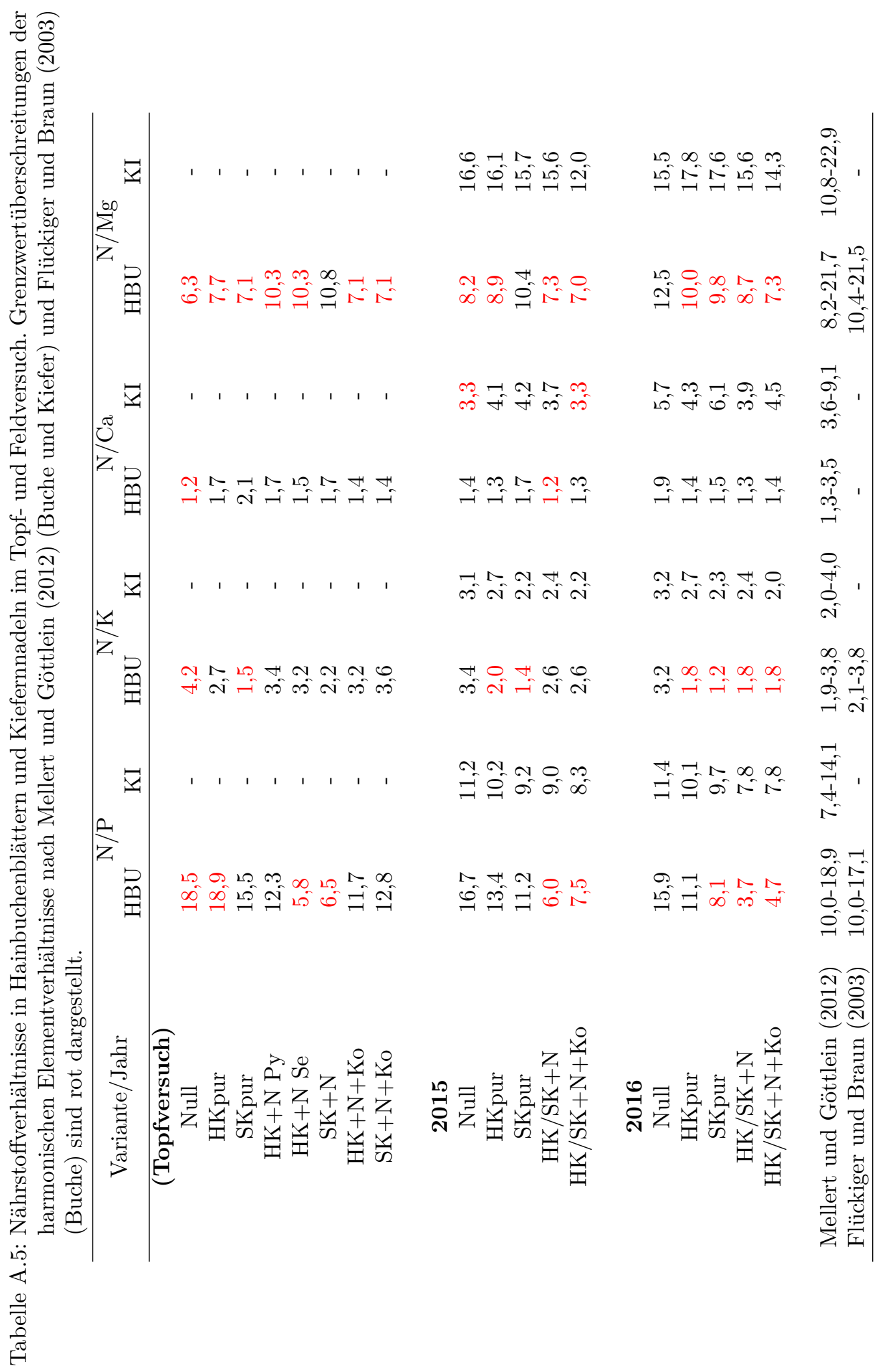




\section{B Abkürzungsverzeichnis}

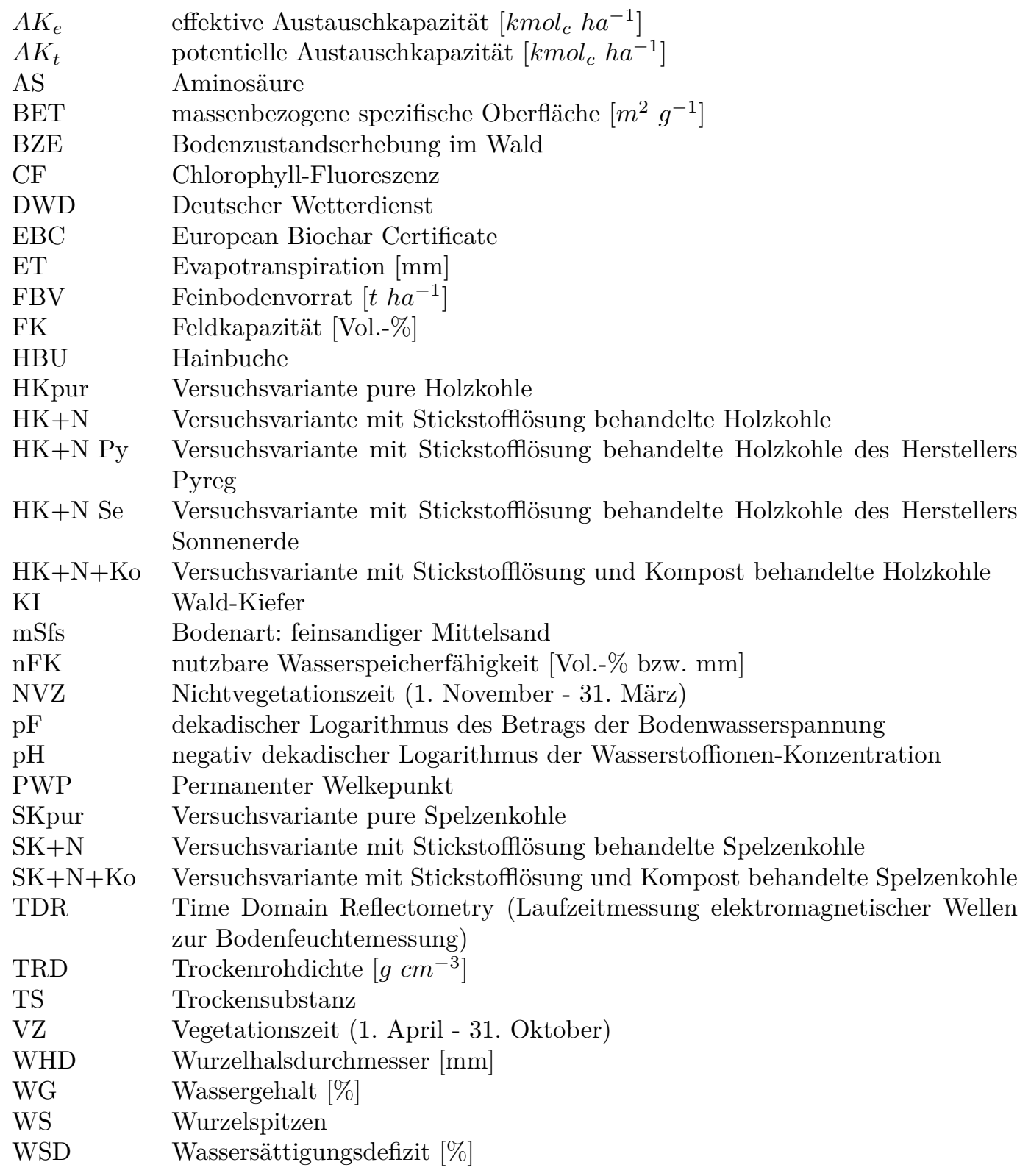

